Supporting Information

\title{
Including bioconcentration kinetics for the prioritization and interpretation of regulatory aquatic toxicity tests of highly hydrophobic chemicals
}

Jung-Hwan Kwon ${ }^{\dagger}$, So-Young Lee ${ }^{\dagger}$, Hyun-Joong Kang ${ }^{\dagger}$, Philipp Mayer ${ }^{\dagger}$, and Beate I. Escher ${ }^{\S \#}$

${ }^{\dagger}$ Division of Environmental Science and Ecological Engineering, Korea University, 145 Anamro, Seongbuk-gu, Seoul 02841, Republic of Korea

Department of Environmental Engineering, Technical University of Denmark, Bygningstorvet B115, DK-2800, Kongens Lyngby, Denmark

${ }^{\S}$ Department of Cell Toxicology, Helmholtz Centre for Environmental Research - UFZ, Permoserstr. 15, DE-04318 Leipzig, Germany

${ }^{\#}$ Environmental Toxicology, Center for Applied Geoscience, Eberhard Eberhard Karls University Tübingen, Hölderlinstr. 12, DE-72074 Tübingen, Germany

*Corresponding author:

junghwakwon@korea.ac.kr

\section{Contents}

Table S1. List of all symbols and abbreviations

Table S2. Summary of melting points of hydrophobic organic substances with $\log \mathrm{K}_{\mathrm{ow}}>4.0$ in the evaluation dataset from SIDS $(n=76)$

Table S3. Uptake rate constants for various aquatic organisms reported in the literature

Table S4. Lipid contents for various aquatic organisms reported in the literature on wet weight basis

Table S5. Summary of reported toxicity test results using algae

Table S6. Summary of reported toxicity test results using invertebrates

Table S7. Summary of reported toxicity test results using fish

Table S8. Outliers of the proposed model with their physico-chemical properties and $\mathrm{LC}_{50}$ or $\mathrm{EC}_{50}$ values 
Table S1. List of all symbols and abbreviations.

\begin{tabular}{|c|c|}
\hline BCR & Lipid-normalized bioconcentration ratio $\left[\mathrm{L} \mathrm{kg}_{\mathrm{lip}}^{-1}\right]$ \\
\hline $\mathrm{BCR}_{\text {crit }}$ & Lipid-normalized critical bioconcentration ratio $\left[\mathrm{L} \mathrm{kg}_{\text {lip }}{ }^{-1}\right]$ \\
\hline BCF & Lipid-normalized bioconcentration factor $\left[\mathrm{L} \mathrm{kg}_{\text {lip }}{ }^{-1}\right]$ \\
\hline CBB & Critical body burden $\left[\mathrm{mmol} \mathrm{kg}_{\text {lip }}{ }^{-1}\right]$ \\
\hline CBR & Critical body residue $\left[\mathrm{mmol} \mathrm{kg}_{\mathrm{lip}}{ }^{-1}\right]$ \\
\hline$C_{w}$ & Concentration of a chemical in water $\left[\mathrm{mmol} \mathrm{L}^{-1}\right]$ \\
\hline$C_{w, \max }$ & Maximum bioavailable concentration of a chemical $\left[\mathrm{mmol} \mathrm{L}^{-1}\right]$ \\
\hline$C_{o}$ & Lipid-normalized concentration of a chemical in organism $\left[\mathrm{mmol} \mathrm{kg}_{\text {lip }}{ }^{-1}\right]$ \\
\hline$C_{o, \max }$ & Maximal lipid-normalized concentration of a chemical in organism [mmol kg $\left.\mathrm{lip}^{-1}\right]$ \\
\hline $\mathrm{EC}_{50}$ & Half maximal effective concentration $\left[\mathrm{mg} \mathrm{L}^{-1}\right]$ \\
\hline$k_{d}$ & Lipid-normalized respiratory depuration rate constant $\left[\mathrm{d}^{-1}\right]$ \\
\hline$k_{g}$ & Growth rate constant for algae $\left[\mathrm{d}^{-1}\right]$ \\
\hline$k_{u}$ & Lipid-normalized respiratory uptake rate constant $\left[\mathrm{L} \mathrm{kg}_{\text {lip }}{ }^{-1} \mathrm{~d}^{-1}\right]$ \\
\hline $\mathrm{LC}_{50}$ & Half maximal lethal concentration $\left[\mathrm{mg} \mathrm{L}^{-1}\right]$ \\
\hline $\log \mathrm{K}_{\mathrm{ow}}$ & Logarithm of octanol-water partition coefficient [-] \\
\hline$M_{o}$ & Lipid mass of an organism $\left[\mathrm{kg}_{\text {lip }}\right]$ \\
\hline OECD & Organization of Economic Coorporation and Development \\
\hline PBT & Persistence, bioaccumulation, and toxicity \\
\hline REACH & Registration, evaluation, authorisation, and restriction of chemicals \\
\hline $\mathrm{S}$ & Water solubility $\left[\mathrm{mmol} \mathrm{L}^{-1}\right]$ \\
\hline$S_{\text {crit }}$ & Critical water solubility $\left[\mathrm{mmol} \mathrm{L}^{-1}\right]$ \\
\hline SIDS & Screening Information Data Set \\
\hline TR & Toxic ratio [-] \\
\hline
\end{tabular}


Table S2. Summary of melting points of hydrophobic organic substances with $\log \mathrm{K}_{\mathrm{ow}}>4.0$ in the evaluation dataset from SIDS $(n=76)$ with a histogram.

\begin{tabular}{|c|c|c|c|}
\hline Chemical name & CAS Reg. no. & $\log K_{\text {ow }}$ & M.P. $\left({ }^{\circ} \mathrm{C}\right)$ \\
\hline Bumetrizole & $3896-11-5$ & 5.55 & 139 \\
\hline Octadecanamide, N,N'-1,2-ethanediylbis- & $110-30-5$ & 13.98 & 139 \\
\hline Methyl laurate & $111-82-0$ & 6.5 & 5.2 \\
\hline 1-Decanol & $112-30-1$ & 5.13 & 6.4 \\
\hline 1-Undecanol & $112-42-5$ & 4.28 & 14.3 \\
\hline 1-Dodecanol & $112-53-8$ & 5.13 & 24 \\
\hline 1-Dodecanethiol & $112-55-0$ & 6.2 & 15.7 \\
\hline 1-Tridecanol & $112-70-9$ & 5.26 & 30.6 \\
\hline 1-Tetradecanol & $112-72-1$ & 5.75 & 39.5 \\
\hline Docosanoic acid & $112-85-6$ & 9.91 & 79.5 \\
\hline 1-Octadecene & $112-88-9$ & 9.04 & 17.7 \\
\hline 1-Octadecanol & $112-92-5$ & 8.22 & 59.8 \\
\hline Triphenyl phosphate & $115-86-6$ & 4.6 & $48-50$ \\
\hline Benzene, 1,1'-oxybis[2,3,4,5,6-pentabromo]- & $1163-19-5$ & 6.27 & $300-305$ \\
\hline 2,6-Dichlorotoluene & $118-69-4$ & 4.25 & 2.8 \\
\hline p-Cresol, 6,6'-di-tert-butyl-2,2'-methylenedi- & $119-47-1$ & 6.25 & $130-131$ \\
\hline Benzene, 1,2,4-trichloro- & $120-82-1$ & 4.02 & 17 \\
\hline 2,6-Di-tert-butyl-p-cresol & $128-37-0$ & 5.1 & 69.8 \\
\hline Phenol, 2,6-bis(1,1-dimethylethyl)- & $128-39-2$ & 4.5 & $36-37$ \\
\hline C.I. Pigment Green 7 & $1328-53-6$ & 17.4 & 480 \\
\hline 2-Butanone, peroxide & $1338-23-4$ & 4.3 & $39.63-126.1$ \\
\hline Ethanol, 2,2'-(octadecyloxidoimino)bis- & $14048-77-2$ & 6.08 & 262.3 \\
\hline 9-Octadecen-1-ol, (9Z)- & $143-28-2$ & 7.5 & $13-19$ \\
\hline 1(3H)-Isobenzofuranone, 6-(dimethylamino)-3,3-bis[4-(dimethylamino)phenyl]- & $1552-42-7$ & 5.27 & $179-180$ \\
\hline 1-Dodecanamine, N,N-dimethyl-, N-oxide & $1643-20-5$ & 4.67 & $130-134$ \\
\hline Phenol, 2,6-bis(1,1-dimethylethyl)-4-(1-methylpropyl)- & $17540-75-9$ & 6.1 & 18.9 \\
\hline Perfluorooctane sulfonic acid & $1763-23-1$ & 4.49 & $>400$ \\
\hline Phenol, 2-(1,1-dimethylethyl)-4,6-dimethyl- & $1879-09-0$ & 4.08 & $21-22$ \\
\hline Octadecyl 3-(3,5-di-tert-butyl-4-hydroxyphenyl)propionate & 2082-79-3 & 13.4 & $49-54$ \\
\hline Ethanone, 1-(5,6,7,8-tetrahydro-3,5,5,6,8,8-hexamethyl-2-naphthalenyl)- & 21145-77-7 & 5.4 & $>54$ \\
\hline 2-Naphthylisobutylether & $2173-57-1$ & 4.65 & 31.75 \\
\hline Peroxide, [1,3(or 1,4)-phenylenebis(1-methylethylidene)]bis[(1,1-dimethylethyl) & $25155-25-3$ & 5.5 & 39.5 \\
\hline Octadecanamine, N,N-dimethyl-, N-oxide & $2571-88-2$ & 7.62 & $130-264$ \\
\hline Benzene, 1,1'-methylenebis(isocyanato- & $26447-40-5$ & 5.22 & $39-43$ \\
\hline Benzenesulfonic acid, dodecyl- & 27176-87-0 & 4.78 & 10 \\
\hline $\begin{array}{l}\text { 1,3,5-Triazine-2,4,6(1H,3H,5H)-trione, 1,3,5-tris[[3,5-bis(1,1-dimethylethyl)-4- } \\
\text { hydroxyphenyl]methyl]- }\end{array}$ & $27676-62-6$ & 5.45 & 349.8 \\
\hline Cyclododecane, 1,2,5,6,9,10-hexabromo- & $3194-55-6$ & 7.74 & $172-184$ \\
\hline Benzene, 1,1'-oxybis-, octabromo deriv. & $32536-52-0$ & 6.29 & $70-257$ \\
\hline 1-Tetradecanamine, N,N-dimethyl-, N-oxide & $3332-27-2$ & 5.66 & 183.3 \\
\hline Perfluorooctanoic Acid & $335-67-1$ & 4.81 & 54.3 \\
\hline Phenol, 5-chloro-2-(2,4-dichlorophenoxy)- & $3380-34-5$ & 4.76 & $54-57.3$ \\
\hline Stannane, dichlorodiocytyl- & $3542-36-7$ & 5.82 & $45-47$ \\
\hline 1-Hexadecanol & $36653-82-4$ & 6.73 & 50 \\
\hline 2,2',3,3'-Tetrachloro-4,4'-diaminodiphenylmethane (TCDAM) & $42240-73-3$ & 5.39 & 186.75 \\
\hline 2-Benzothiazolesulfenamide, N,N-dicyclohexyl- & $4979-32-2$ & 4.8 & 99 \\
\hline Neodecanoic acid, ethenyl ester & $51000-52-3$ & 4.9 & 7.2 \\
\hline Pigment Yellow 13 & $5102-83-0$ & 8.11 & 350 \\
\hline Disperse Yellow-42 & $5124-25-4$ & 4.6 & 159.85 \\
\hline Cyclohexane, 1,1'-methylenebis[4-isocyanato- & $5124-30-1$ & 6.11 & 15 \\
\hline Decanedioic acid, bis(2,2,6,6-tetramethyl-4-piperidinyl) ester & $52829-07-9$ & 6.5 & 210 \\
\hline Cyclotrisiloxane, hexamethyl- & $541-05-9$ & 4.47 & 64 \\
\hline $\begin{array}{l}\text { Butanamide, 2,2'-[(3,3'-dichloro[1,1'-biphenyl]-4,4'-diyl)bis(azo)]bis[N-(4-chloro-2,5- } \\
\text { dimethoxyphenyl)-3-oxo- }\end{array}$ & $5567-15-7$ & 7.54 & 400 \\
\hline Dimethylin bis[2-ethylhexylthioglycolate] & $57583-35-4$ & 8.48 & 90 \\
\hline Phosphine, triphenyl- & $603-35-0$ & 5.69 & 80.5 \\
\hline Amine oxides, cocoalkyldimethyl & 61788-90-7 & 4.67 & $125-136$ \\
\hline 1-Pentadecanol & $629-76-5$ & 6.24 & $44-46$ \\
\hline
\end{tabular}


1-Eicosanol

\begin{tabular}{ccc}
$629-96-9$ & 8.7 & $64-68$ \\
$6358-85-6$ & 7.05 & 320 \\
$6386-38-5$ & 5.5 & 66.1 \\
$661-19-8$ & 9.68 & 72.5 \\
$6683-19-8$ & 5 & 116.5 \\
$7128-91-8$ & 6.64 & 198.7 \\
$76-83-5$ & 5.25 & 113.5 \\
$793-24-8$ & 4.68 & 50 \\
$79-92-5$ & 4.12 & $45-46$ \\
$79-94-7$ & 5.9 & $181-182$ \\
$80-43-3$ & 5.6 & 39.8 \\
$81-14-1$ & 4.3 & $135-137$ \\
$81-15-2$ & 4.9 & $112-114$ \\
$818-08-6$ & 5.33 & 105 \\
$84-51-5$ & 4.37 & 108.8 \\
$88-60-8$ & 4.11 & 213 \\
$92-52-4$ & 4.01 & 69 \\
$93962-62-0$ & 5.86 & $125-136$ \\
$95-33-0$ & 4.93 & $97.5-105$ \\
\hline
\end{tabular}

Metilox

1-Docosanol

Pentaerythritol tetrakis[3-(3,5-di-tert-butyl-4-hydroxypheny

hexadecyldimethylamine $\mathrm{N}$-oxide

Triphenylmethyl chloride

p-Phenylenediamine, N-(1,3-dimethylbutyl)-N'-phenyl-

Camphene

2,2',6,6'-Tetrabromo-4,4'-isopropylidenediphenol

Peroxide, bis(1-methyl-1-phenylethyl)-

4-tert-Butyl-2,6-dimethyl-3,5-dinitroacetophenone (Musk ketone)

5-tert-Butyl-2,4,6-trinitro-m-xylene (Musk xylene)

Stannane, dibutyloxo-

Anthraquinone, 2-ethyl-

m-Cresol, 6-tert-butyl-

$1,1^{\prime}$-Biphenyl

Ethanol, 2,2'-[(9Z)-9-octadecenyloxidoimino]bis-

2-Benzothiazolesulfenamide, N-cyclohexyl-

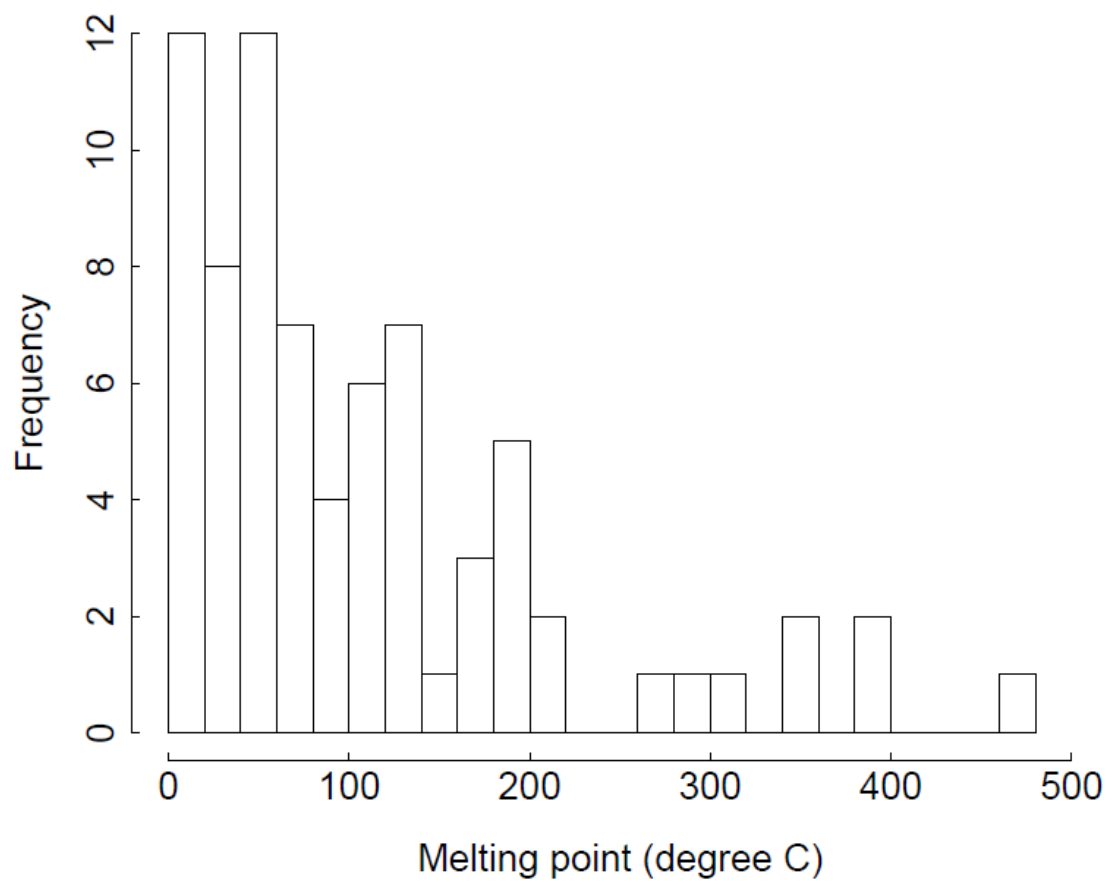


Table S3. Uptake rate constants $\left(\mathrm{k}_{\mathrm{u}}\right)$ for various aquatic organisms reported in the literature.

\begin{tabular}{|c|c|c|c|c|c|c|c|c|}
\hline Organism & Species & $\%$ lipid & Size (g) & Chemicals & $\log \mathrm{K}_{\mathrm{ow}}$ & $\begin{array}{c}\mathrm{k}_{\mathrm{u}} \\
\left(\mathrm{L} \mathrm{kg}_{\mathrm{ww}}{ }^{-1} \mathrm{~d}^{-1}\right)\end{array}$ & $\begin{array}{c}\mathrm{k}_{\mathrm{u}} \\
\left(\mathrm{L} \mathrm{kg}_{\text {lipid }}{ }^{-1} \mathrm{~d}^{-1}\right)\end{array}$ & Reference \\
\hline \multirow[t]{5}{*}{ Algae } & Pseudokirchneriella subcapita & n.a. & n.a. & tetrachlorobenzene & 4.6 & 51840 & $2160000^{\mathrm{a}}$ & [1] \\
\hline & Pseudokirchneriella subcapita & n.a. & n.a. & anthracene & 4.49 & 190080 & $7920000^{\mathrm{a}}$ & [1] \\
\hline & Pseudokirchneriella subcapita & n.a. & n.a. & DDT & 5.7 & 760320 & $31680000^{\mathrm{a}}$ & [1] \\
\hline & Pseudokirchneriella subcapita & n.a. & n.a. & hexachlorobenzene & 5.64 & 155520 & $6480000^{\mathrm{a}}$ & [1] \\
\hline & Pseudokirchneriella subcapita & n.a. & n.a. & hexachlorobiphenvl & 7.08 & 501120 & $20880000^{\mathrm{a}}$ & [1] \\
\hline \multirow[t]{26}{*}{ Invertebrate } & Hyulella aztecu & n.a. & n.a. & mirex & 6.89 & 5152 & $271158^{\mathrm{a}}$ & [2] \\
\hline & Hyulella aztecu & n.a. & n.a & mirex & 6.89 & 4246 & $223474^{\mathrm{a}}$ & [2] \\
\hline & Hyulella aztecu & n.a. & n.a & $\operatorname{mirex}$ & 6.89 & 4318 & $227263^{\mathrm{a}}$ & [2] \\
\hline & Hyulella aztecu & n.a. & n.a & $\operatorname{mirex}$ & 6.89 & 7414 & $390211^{\mathrm{a}}$ & [2] \\
\hline & Hyulella aztecu & n.a. & n.a & mirex & 6.89 & 4813 & $253316^{\mathrm{a}}$ & [2] \\
\hline & Hyulella aztecu & n.a. & n.a & $\operatorname{mirex}$ & 6.89 & 5680 & $298947^{\mathrm{a}}$ & [2] \\
\hline & Crungonyx pseudogracilis & n.a. & n.a & $\operatorname{mirex}$ & 6.89 & 2454 & $129158^{\mathrm{a}}$ & [2] \\
\hline & Crungonyx pseudogracilis & n.a. & n.a & mirex & 6.89 & 3047 & $160368^{\mathrm{a}}$ & [2] \\
\hline & Crungonyx pseudogracilis & n.a. & n.a & mirex & 6.89 & 2262 & $119053^{\mathrm{a}}$ & {$[2]$} \\
\hline & Asellus aquaticus & 0.3 & $4-20 \mathrm{~mm}$ & anthracene & 4.54 & 950 & $279411^{\mathrm{b}}$ & [3] \\
\hline & Asellus aquaticus & 0.3 & $4-20 \mathrm{~mm}$ & phenanthrene & 4.57 & 1500 & $441176^{\mathrm{b}}$ & [3] \\
\hline & Asellus aquaticus & 0.3 & $4-20 \mathrm{~mm}$ & pyrene & 5.18 & 640 & $188235^{\mathrm{b}}$ & [3] \\
\hline & Asellus aquaticus & 0.3 & $4-20 \mathrm{~mm}$ & benzo[a]pyrene & 6.04 & 4800 & $1411764^{\mathrm{b}}$ & {$[3]$} \\
\hline & Asellus aquaticus & 0.3 & 4-20 mm & benzo[e]pyrene & 6.04 & 4300 & $1264705^{\mathrm{b}}$ & [3] \\
\hline & Asellus aquaticus & 0.3 & $4-20 \mathrm{~mm}$ & benzo[ghi]perylene & 7.04 & 1380 & $405882^{\mathrm{b}}$ & [3] \\
\hline & Daphnia magna & n.a. & 0.0016 & atrazine & 2.61 & 53.04 & $2792^{\mathrm{a}}$ & [4] \\
\hline & Daphnia magna & n.a. & 0.0016 & atrazine & 2.61 & 42.72 & $2248^{\mathrm{a}}$ & {$[4]$} \\
\hline & Daphnia magna & n.a. & 0.0016 & atrazine & 2.61 & 41.76 & $2198^{\mathrm{a}}$ & [4] \\
\hline & Daphnia magna & n.a. & 0.0016 & atrazine & 2.61 & 53.28 & $2804^{\mathrm{a}}$ & [4] \\
\hline & Pontoporeia hoyi & $20-50$ & 0.004-0.009 & Anthracene & 4.45 & 3146.4 & $165600^{\mathrm{a}}$ & [5] \\
\hline & Pontoporeia hoyi & $20-50$ & 0.004-0.009 & Benz(a)anthracene & 5.9 & 3326.4 & $175074^{\mathrm{a}}$ & [5] \\
\hline & Pontoporeia hoyi & $20-50$ & $0.004-0.009$ & Benzo(a)pyrene & 6.5 & 2803.2 & $147537^{\mathrm{a}}$ & [5] \\
\hline & Pontoporeia hoyi & $20-50$ & 0.004-0.009 & Biphenyl & 3.2 & 2289.6 & $120505^{\mathrm{a}}$ & [5] \\
\hline & Pontoporeia hoyi & $20-50$ & 0.004-0.009 & Phenanthrene & 4.16 & 3096 & $162947^{\mathrm{a}}$ & [5] \\
\hline & Pontoporeia hoyi & $20-50$ & $0.004-0.009$ & Pyrene & 5.2 & 4780.8 & $251621^{\mathrm{a}}$ & [5] \\
\hline & Pontoporeia hoyi & $20-50$ & $0.004-0.009$ & Tetrachlorobiphenyl & 4.4 & 3237.6 & $170400^{\mathrm{a}}$ & [5] \\
\hline \multirow[t]{41}{*}{ Fish } & trout $(S$. trutta $)$ & n.a. & 40 & PCB6 & 5.06 & 1500 & $24550^{\mathrm{a}}$ & {$[6]$} \\
\hline & trout (S. trutta) & n.a. & 40 & PCB18 & 5.24 & 1100 & $18003^{\mathrm{a}}$ & [6] \\
\hline & trout (S. trutta) & n.a. & 40 & PCB19 & 5.02 & 700 & $11457^{\mathrm{a}}$ & [6] \\
\hline & trout (S. trutta) & n.a. & 40 & PCB22 & 5.58 & 900 & $14730^{\mathrm{a}}$ & [6] \\
\hline & trout (S. trutta) & n.a. & 40 & PCB25 & 5.67 & 700 & $11457^{\mathrm{a}}$ & [6] \\
\hline & trout (S. trutta) & n.a. & 40 & PCB26 & 5.66 & 700 & $11457^{\mathrm{a}}$ & [6] \\
\hline & trout $(S$. trutta $)$ & n.a. & 40 & РCB28 & 5.67 & 1000 & $16367^{\mathrm{a}}$ & [6] \\
\hline & trout (S. trutta) & n.a. & 40 & PCB31 & 5.67 & 800 & $13093^{\mathrm{a}}$ & {$[6]$} \\
\hline & trout (S. trutta) & n.a. & 40 & PCB40 & 5.66 & 700 & $11457^{\mathrm{a}}$ & [6] \\
\hline & trout (S. trutta) & n.a. & 40 & PCB41 & 5.69 & 600 & $9820^{\mathrm{a}}$ & [6] \\
\hline & trout (S. trutta) & n.a. & 40 & PCB42 & 5.76 & 700 & $11457^{\mathrm{a}}$ & [6] \\
\hline & trout (S. trutta) & n.a. & 40 & PCB44 & 5.75 & 900 & $14730^{\mathrm{a}}$ & [6] \\
\hline & trout (S. trutta) & n.a. & 40 & PCB45 & 5.53 & 900 & $14730^{\mathrm{a}}$ & [6] \\
\hline & trout (S. trutta) & n.a. & 40 & PCB46 & 5.53 & 400 & $6547^{\mathrm{a}}$ & [6] \\
\hline & trout (S. trutta) & n.a. & 40 & PCB47 & 5.85 & 900 & $14730^{\mathrm{a}}$ & [6] \\
\hline & trout (S. trutta) & n.a. & 40 & PCB48 & 5.78 & 900 & $14730^{\mathrm{a}}$ & [6] \\
\hline & trout (S. trutta) & n.a. & 40 & PCB49 & 5.85 & 1100 & $18003^{\mathrm{a}}$ & [6] \\
\hline & trout (S. trutta) & n.a. & 40 & PCB51 & 5.63 & 500 & $8183^{\mathrm{a}}$ & [6] \\
\hline & trout (S. trutta) & n.a. & 40 & PCB52 & 5.84 & 1000 & $16367^{\mathrm{a}}$ & [6] \\
\hline & trout (S. trutta) & n.a. & 40 & PCB63 & 6.17 & 600 & $9820^{\mathrm{a}}$ & [6] \\
\hline & trout (S. trutta) & n.a. & 40 & PCB64 & 5.95 & 900 & $14730^{\mathrm{a}}$ & [6] \\
\hline & trout (S. trutta) & n.a. & 40 & PCB67 & 6.2 & 600 & $9820^{\mathrm{a}}$ & [6] \\
\hline & trout (S. trutta) & n.a. & 40 & PCB70 & 6.2 & 900 & $14730^{\mathrm{a}}$ & [6] \\
\hline & trout (S. trutta) & n.a. & 40 & PCB74 & 6.2 & 700 & $11457^{\mathrm{a}}$ & [6] \\
\hline & trout (S. trutta) & n.a. & 40 & PCB82 & 6.2 & 500 & $8183^{\mathrm{a}}$ & [6] \\
\hline & trout (S. trutta) & n.a. & 40 & PCB83 & 6.26 & 500 & $8183^{\mathrm{a}}$ & [6] \\
\hline & trout (S. trutta) & n.a. & 40 & PCB84 & 6.04 & 400 & $6547^{\mathrm{a}}$ & [6] \\
\hline & trout (S. trutta) & n.a. & 40 & PCB87 & 6.29 & 700 & $11457^{\mathrm{a}}$ & [6] \\
\hline & trout (S. trutta) & n.a. & 40 & PCB91 & 6.13 & 500 & $8183^{\mathrm{a}}$ & [6] \\
\hline & trout (S. trutta) & n.a. & 40 & PCB92 & 6.35 & 500 & $8183^{\mathrm{a}}$ & [6] \\
\hline & trout (S. trutta) & n.a. & 40 & PCB97 & 6.29 & 600 & $9820^{\mathrm{a}}$ & [6] \\
\hline & trout $(S$. trutta $)$ & n.a. & 40 & РСB99 & 6.39 & 500 & $8183^{\mathrm{a}}$ & [6] \\
\hline & trout $(S$. trutta $)$ & n.a. & 40 & PCB101 & 6.38 & 1400 & $22913^{\mathrm{a}}$ & [6] \\
\hline & trout $(S$. trutta $)$ & n.a. & 40 & PCB105 & 6.65 & 600 & $9820^{\mathrm{a}}$ & [6] \\
\hline & trout (S. trutta) & n.a. & 40 & PCB107 & 6.71 & 600 & $9820^{\mathrm{a}}$ & [6] \\
\hline & trout $(S$. trutta $)$ & n.a. & 40 & PCB110 & 6.48 & 600 & $9820^{\mathrm{a}}$ & [6] \\
\hline & trout $(S$. trutta $)$ & n.a. & 40 & PCB118 & 6.74 & 600 & $9820^{\mathrm{a}}$ & [6] \\
\hline & trout (S. trutta) & n.a. & 40 & PCB119 & 6.58 & 600 & $9820^{\mathrm{a}}$ & [6] \\
\hline & trout $(S$. trutta $)$ & n.a. & 40 & PCB128 & 6.74 & 500 & $8183^{\mathrm{a}}$ & [6] \\
\hline & trout $(S$. trutta $)$ & n.a. & 40 & PCB129 & 6.73 & 400 & $6547^{\mathrm{a}}$ & [6] \\
\hline & trout $(S$. trutta $)$ & n.a. & 40 & PCB130 & 6.8 & 500 & $8183^{\mathrm{a}}$ & {$[6]$} \\
\hline
\end{tabular}


(Table S3. continued)

\begin{tabular}{|c|c|c|c|c|c|c|c|c|}
\hline Organism & Species & $\%$ lipid & Size (g) & Chemicals & $\log \mathrm{K}_{\mathrm{ow}}$ & $\begin{array}{c}\mathrm{k}_{\mathrm{u}} \\
\left(\mathrm{L} \mathrm{kg}_{\mathrm{ww}}{ }^{-1} \mathrm{~d}^{-1}\right)\end{array}$ & 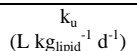 & Reference \\
\hline \multirow[t]{83}{*}{ Fish } & trout $(S$. trutta $)$ & n.a. & 40 & PCB134 & 6.55 & $\frac{\left(L \mathrm{Rgww}^{2} \text { a) }\right.}{700}$ & (L Rlipid a & [6] \\
\hline & trout (S. trutta) & n.a. & 40 & PCB136 & 6.22 & 500 & $8183^{\mathrm{a}}$ & [6] \\
\hline & trout $(S$. trutta $)$ & n.a. & 40 & PCB137 & 6.83 & 400 & $6547^{\mathrm{a}}$ & {$[6]$} \\
\hline & trout $(S$. trutta $)$ & n.a. & 40 & PCB138 & 6.83 & 500 & $8183^{\mathrm{a}}$ & [6] \\
\hline & trout $(S$. trutta $)$ & n.a. & 40 & PCB141 & 6.82 & 500 & $8183^{\mathrm{a}}$ & [6] \\
\hline & trout $(S$. trutta $)$ & n.a. & 40 & PCB146 & 6.89 & 700 & $11457^{\mathrm{a}}$ & [6] \\
\hline & trout $(S$. trutta $)$ & n.a. & 40 & PCB149 & 6.67 & 600 & $9820^{\mathrm{a}}$ & [6] \\
\hline & trout $(S$. trutta $)$ & n.a. & 40 & PCB151 & 6.64 & 700 & $11457^{\mathrm{a}}$ & [6] \\
\hline & trout $(S$. trutta $)$ & n.a. & 40 & PCB153 & 6.92 & 700 & $11457^{\mathrm{a}}$ & [6] \\
\hline & trout (S. trutta) & n.a. & 40 & PCB158 & 7.02 & 400 & $6547^{\mathrm{a}}$ & [6] \\
\hline & trout $(S$. trutta $)$ & n.a. & 40 & PCB172 & 7.33 & 100 & $1637^{\mathrm{a}}$ & [6] \\
\hline & trout $(S$. trutta $)$ & n.a. & 40 & PCB174 & 7.11 & 400 & $6547^{\mathrm{a}}$ & [6] \\
\hline & trout $(S$. trutta $)$ & n.a. & 40 & PCB176 & 6.76 & 300 & $4910^{\mathrm{a}}$ & [6] \\
\hline & trout $(S$. trutta $)$ & n.a. & 40 & PCB 177 & 7.08 & 400 & $6547^{\mathrm{a}}$ & [6] \\
\hline & trout $(S$. trutta $)$ & n.a. & 40 & PCB178 & 7.14 & 600 & $9820^{\mathrm{a}}$ & [6] \\
\hline & trout $(S$. trutta $)$ & n.a. & 40 & PCB179 & 6.73 & 400 & $6547^{\mathrm{a}}$ & [6] \\
\hline & trout $(S$. trutta $)$ & n.a. & 40 & PCB 180 & 7.36 & 400 & $6547^{\mathrm{a}}$ & [6] \\
\hline & trout $(S$. trutta $)$ & n.a. & 40 & PCB 183 & 7.2 & 400 & $6547^{\mathrm{a}}$ & [6] \\
\hline & trout $(S$. trutta $)$ & n.a. & 40 & PCB187 & 7.17 & 500 & $8183^{\mathrm{a}}$ & [6] \\
\hline & trout $(S . t r u t t a)$ & n.a. & 40 & PCB194 & 7.8 & 200 & $3273^{\mathrm{a}}$ & [6] \\
\hline & trout $(S$. trutta $)$ & n.a. & 40 & PCB199 & 7.62 & 300 & $4910^{\mathrm{a}}$ & [6] \\
\hline & rainbow trout & $10.0 \pm 2.1$ & $8.8 \pm 1.5$ & Aldrin & 5.3 & 13.6 & $136^{\mathrm{b}}$ & [7] \\
\hline & rainbow trout & $10.0 \pm 2.1$ & $8.8 \pm 1.5$ & Heptachlor epoxide & 5.4 & 20.4 & $204^{\mathrm{b}}$ & [7] \\
\hline & rainbow trout & $10.0 \pm 2.1$ & $8.8 \pm 1.5$ & 4,4'-DDT & 5.7 & 27.3 & $273^{\mathrm{b}}$ & [7] \\
\hline & rainbow trout & $10.0 \pm 2.1$ & $8.8 \pm 1.5$ & Hexachlorobenzene & 6.2 & 31.8 & $318^{\mathrm{b}}$ & [7] \\
\hline & rainbow trout (S. gairdneri) & $6.84 \pm 0.93$ & $240 \pm 45$ & PCB 9 & 5.2 & 280 & $40936^{\mathrm{b}}$ & [8] \\
\hline & rainbow trout (S. gairdneri) & $6.84 \pm 0.93$ & $240 \pm 45$ & PCB 5 & 5.2 & 300 & $43860^{\mathrm{b}}$ & {$[8]$} \\
\hline & rainbow trout (S. gairdneri) & $6.84 \pm 0.93$ & $240 \pm 45$ & PCB 14 & 5.2 & 310 & $45322^{\mathrm{b}}$ & [8] \\
\hline & rainbow trout (S. gairdneri) & $6.84 \pm 0.93$ & $240 \pm 45$ & PCB 18 & 5.6 & 300 & $43860^{\mathrm{b}}$ & [8] \\
\hline & rainbow trout (S. gairdneri) & $6.84 \pm 0.93$ & $240 \pm 45$ & PCB 52 & 5.8 & 280 & $40936^{\mathrm{b}}$ & {$[8]$} \\
\hline & rainbow trout (S. gairdneri) & $6.84 \pm 0.93$ & $240 \pm 45$ & PCB 40 & 5.8 & 320 & $46784^{\mathrm{b}}$ & {$[8]$} \\
\hline & rainbow trout (S. gairdneri) & $6.84 \pm 0.93$ & $240 \pm 45$ & PCB 155 & 6.7 & 60 & $8772^{\mathrm{b}}$ & {$\left[\begin{array}{l}{[0]} \\
{[8]}\end{array}\right.$} \\
\hline & rainbow trout $(S$. gairdneri $)$ & $6.84 \pm 0.93$ & $240 \pm 45$ & PCB 101 & 6.1 & 180 & $26316^{\mathrm{b}}$ & [8] \\
\hline & rainbow trout (S. gairdneri) & $6.84 \pm 0.93$ & $240 \pm 45$ & $\mathrm{p}, \mathrm{p}^{\prime}-\mathrm{DDE}$ & 5.7 & 170 & $24854^{\mathrm{b}}$ & {$[8]$} \\
\hline & rainbow trout (S. gairdneri) & $6.84 \pm 0.93$ & $240 \pm 45$ & lindane & 3.7 & 130 & $19006^{\mathrm{b}}$ & [8] \\
\hline & rainbow trout (S. gairdneri) & $6.84 \pm 0.93$ & $240 \pm 45$ & $\gamma$-chlor & 6 & 340 & $49708^{b}$ & [8] \\
\hline & rainbow trout (S. gairdneri) & $6.84 \pm 0.93$ & $240 \pm 45$ & $\alpha$-chlor & 6 & 340 & $49708^{\mathrm{b}}$ & {$[8]$} \\
\hline & rainbow trout $(S$. gairdneri) & $6.84 \pm 0.93$ & $240 \pm 45$ & mirex & 6.9 & 8.5 & $1243^{\mathrm{b}}$ & [8] \\
\hline & turbot $($ S. maximus $)$ & 3.0 & 0.89 & Naphthalene & 3.34 & 396 & 11000 & [9] \\
\hline & turbot (S. maximus) & 3.0 & 0.89 & C1-naphthalene & 3.86 & 975 & 27300 & [9] \\
\hline & turbot (S. maximus) & 3.0 & 0.89 & C2-naphthalene & 3.91 & 1,232 & 34500 & [9] \\
\hline & turbot (S. maximus) & 3.0 & 0.89 & C3-naphthalene & 4.9 & 606 & 16900 & [9] \\
\hline & turbot (S. maximus) & 3.0 & 0.89 & Acenaphthylene & 4.08 & 1,055 & $35166^{\mathrm{b}}$ & [9] \\
\hline & turbot (S. maximus) & 3.0 & 0.89 & Acenaphthene & 4.08 & 525 & $17500^{\mathrm{b}}$ & [9] \\
\hline & turbot (S. maximus) & 3.0 & 0.89 & Fluorene & 4.22 & 885 & 24400 & [9] \\
\hline & turbot (S. maximus) & 3.0 & 0.89 & Anthracene & 4.53 & 758 & $25267^{\mathrm{b}}$ & [9] \\
\hline & turbot (S. maximus) & 3.0 & 0.89 & Phenanthrene & 4.53 & 646 & 17800 & [9] \\
\hline & turbot (S. maximus) & 3.0 & 0.89 & C1-phenanthrene & 5.15 & 144 & 3800 & [9] \\
\hline & turbot (S. maximus) & 3.0 & 0.89 & C2-phenanthrene & 5.51 & 15 & 400 & [9] \\
\hline & turbot (S. maximus) & 3.0 & 0.89 & Dibenzothiophene & 4.38 & 912 & 24700 & [9] \\
\hline & turbot (S. maximus) & 3.0 & 0.89 & Fluoranthene & 5.24 & 210 & 5600 & [9] \\
\hline & turbot (S. maximus) & 3.0 & 0.89 & Chrysene & 5.77 & 64 & 1200 & [9] \\
\hline & zebrafish (B. rerio) & 2.9 & $0.243 \pm 0.048$ & PCB 9 & 5.06 & 2760 & $94520^{\mathrm{b}}$ & [10] \\
\hline & zebrafish (B. rerio) & 2.9 & $0.243 \pm 0.048$ & PCB 18 & 5.24 & 3760 & $128767^{\mathrm{b}}$ & {$[10]$} \\
\hline & zebrafish (B. rerio) & 2.9 & $0.243 \pm 0.048$ & PCB 31 & 5.67 & 3950 & $135274^{\mathrm{b}}$ & {$[10]$} \\
\hline & zebrafish (B. rerio) & 2.9 & $0.243 \pm 0.048$ & PCB 44 & 5.75 & 3200 & $109589^{\mathrm{b}}$ & {$[10]$} \\
\hline & zebrafish (B. rerio) & 2.9 & $0.243 \pm 0.048$ & PCB 49 & 5.85 & 3200 & $109589^{\mathrm{b}}$ & {$[10]$} \\
\hline & zebrafish (B. rerio) & 2.9 & $0.243 \pm 0.048$ & PCB 52 & 5.84 & 3230 & $110616^{\mathrm{b}}$ & {$[10]$} \\
\hline & zebrafish (B. rerio) & 2.9 & $0.243 \pm 0.048$ & PCB 54 & 5.21 & 2740 & $93836^{\mathrm{b}}$ & {$[10]$} \\
\hline & zebrafish (B. rerio) & 2.9 & $0.243 \pm 0.048$ & PCB 70 & 6.2 & 3340 & $11438^{\mathrm{b}}$ & {$[10]$} \\
\hline & zebrafish (B. rerio) & 2.9 & $0.243 \pm 0.048$ & PCB 77 & 6.36 & 5160 & $176712^{\mathrm{b}}$ & {$[10]$} \\
\hline & zebrafish (B. rerio) & 2.9 & $0.243 \pm 0.048$ & PCB 87 & 6.29 & 3360 & $115068^{\mathrm{b}}$ & {$[10]$} \\
\hline & zebrafish (B. rerio) & 2.9 & $0.243 \pm 0.048$ & PCB 97 & 6.29 & 3400 & $116438^{\mathrm{b}}$ & {$[10]$} \\
\hline & zebrafish (B. rerio) & 2.9 & $0.243 \pm 0.048$ & PCB 101 & 6.38 & 3850 & $131849^{\mathrm{b}}$ & {$[10]$} \\
\hline & zebrafish (B. rerio) & 2.9 & $0.243 \pm 0.048$ & PCB 126 & 6.89 & 6490 & $222260^{\mathrm{b}}$ & {$[10]$} \\
\hline & zebrafish (B. rerio) & 2.9 & $0.243 \pm 0.048$ & PCB 128 & 6.74 & 4970 & $170205^{\mathrm{b}}$ & {$[10]$} \\
\hline & zebrafish (B. rerio) & 2.9 & $0.243 \pm 0.048$ & PCB 136 & 6.22 & 4300 & $147260^{\mathrm{b}}$ & {$[10]$} \\
\hline & zebrafish (B. rerio) & 2.9 & $0.243 \pm 0.048$ & PCB 138 & 6.83 & 4770 & $163356^{\mathrm{b}}$ & {$[10]$} \\
\hline & zebrafish (B. rerio) & 2.9 & $0.243 \pm 0.048$ & PCB 141 & 6.82 & 4910 & $168151^{\mathrm{b}}$ & {$[10]$} \\
\hline & zebrafish (B. rerio) & 2.9 & $0.243 \pm 0.048$ & PCB 151 & $\begin{array}{l}0.02 \\
6.64\end{array}$ & 4220 & $144520^{\mathrm{b}}$ & {$[10]$} \\
\hline & zebrafish (B. rerio) & 2.9 & $0.243 \pm 0.048$ & PCB 153 & 6.92 & 4660 & $159589^{\mathrm{b}}$ & {$[10]$} \\
\hline & zebrafish (B. rerio) & 2.9 & $0.243 \pm 0.048$ & PCB 169 & 7.42 & 7200 & $246575^{\mathrm{b}}$ & {$[10]$} \\
\hline & zebrafish (B. rerio) & 2.9 & $0.243 \pm 0.048$ & PCB 183 & 7.2 & 6000 & $205479^{\mathrm{b}}$ & {$[10]$} \\
\hline & zebrafish (B. rerio) & 2.9 & $0.243 \pm 0.048$ & PCB 185 & 7.11 & 5810 & $198972^{\mathrm{b}}$ & {$[10]$} \\
\hline & zebrafish (B. rerio) & 2.9 & $0.243 \pm 0.048$ & PCB 194 & 7.8 & 5640 & $193150^{\mathrm{b}}$ & {$[10]$} \\
\hline & zebrafish (B. rerio) & $\begin{array}{l}2.9 \\
2.9\end{array}$ & $\begin{array}{l}0.24 J \pm 0.040 \\
0.243 \pm 0.048\end{array}$ & PCB 195 & $\begin{array}{l}1.0 \\
7.56\end{array}$ & $\begin{array}{l}5040 \\
5930\end{array}$ & $203082^{\mathrm{b}}$ & {$[10]$} \\
\hline & zebrafish (B. rerio) & 2.9 & $0.243 \pm 0.048$ & PCB 198 & 7.62 & 5950 & $203767^{\mathrm{b}}$ & {$[10]$} \\
\hline & zebrafish (B. rerio) & 2.9 & $0.243 \pm 0.048$ & PCB 202 & 7.02 & 5070 & $173630^{\mathrm{b}}$ & {$[10]$} \\
\hline & zebrafish (B. rerio) & 2.9 & $0.243 \pm 0.048$ & PCB 206 & 8.09 & 4940 & $169178^{\mathrm{b}}$ & {$[10]$} \\
\hline & zebrafish (B. rerio) & 2.9 & $0.243 \pm 0.048$ & PCB 209 & 8.48 & 3640 & $124657^{\mathrm{b}}$ & {$[10]$} \\
\hline & zebrafish (B. rerio) & $6.62 \pm 2.31$ & $0.213 \pm 0.041$ & $\alpha-\mathrm{HCH}$ & $\begin{array}{l}0.40 \\
3.82\end{array}$ & 1200 & $18126^{\mathrm{b}}$ & {$[11]$} \\
\hline & zebrafish (B. rerio) & $6.62 \pm 2.31$ & $0.213 \pm 0.041$ & $\beta-\mathrm{HCH}$ & 3.8 & 1015.2 & $15335^{\mathrm{b}}$ & [11] \\
\hline & zebrafish (B. rerio) & $6.62 \pm 2.31$ & $0.213 \pm 0.041$ & $\gamma-\mathrm{HCH}$ & 3.72 & 1219.2 & $18417^{\mathrm{b}}$ & [11] \\
\hline
\end{tabular}


(Table S3. continued)

\begin{tabular}{|c|c|c|c|c|c|c|c|c|}
\hline Organism & Species & $\%$ lipid & Size (g) & Chemicals & $\log \mathrm{K}_{\mathrm{ow}}$ & $\begin{array}{c}\mathrm{k}_{\mathrm{u}} \\
\left(\mathrm{L} \mathrm{kg}_{\mathrm{ww}}{ }^{-1} \mathrm{~d}^{-1}\right)\end{array}$ & $\begin{array}{c}\mathrm{k}_{\mathrm{u}} \\
\left(\mathrm{L} \mathrm{kg}_{\text {lipid }}{ }^{-1} \mathrm{~d}^{-1}\right)\end{array}$ & Reference \\
\hline \multirow[t]{73}{*}{ Fish } & zebrafish $($ B. rerio $)$ & $6.62 \pm 2.31$ & $0.213 \pm 0.041$ & $\delta$-HCH & 4.14 & 1320 & $19940^{\mathrm{b}}$ & [11] \\
\hline & goldfish (C. auratus) & 6.3 & 4.52 & PCB 9 & 5.06 & 920 & $14603^{\mathrm{b}}$ & {$[12]$} \\
\hline & goldfish (C. auratus) & 6.3 & 4.52 & PCB 18 & 5.24 & 950 & $15079^{\mathrm{b}}$ & {$[12]$} \\
\hline & goldfish (C. auratus) & 6.3 & 4.52 & PCB 31 & 5.67 & 890 & $14127^{\mathrm{b}}$ & {$[12]$} \\
\hline & goldfish (C. auratus) & 6.3 & 4.52 & PCB 52 & 5.84 & 740 & $11746^{\mathrm{b}}$ & {$[12]$} \\
\hline & goldfish (C. auratus) & 6.3 & 4.52 & PCB 70 & 6.2 & 420 & $6667^{\mathrm{b}}$ & [12] \\
\hline & $\begin{array}{l}\text { bluegill sunfish (Lepomis } \\
\text { macrochirus) }\end{array}$ & $1.5 \pm 0.2$ & $0.1-0.5$ & $1,2,3,5$-Tetrachlorobenzene & 4.67 & & 1776 & {$[13]$} \\
\hline & $\begin{array}{l}\text { bluegill sunfish (Lepomis } \\
\text { macrochirus) }\end{array}$ & $1.5 \pm 0.2$ & $0.1-0.5$ & Pentachlorobenzene & 5.2 & & 2640 & [13] \\
\hline & $\begin{array}{l}\text { rainbow trout (Salmo } \\
\text { gairdneri) }\end{array}$ & 2.9 & $0.1-0.6$ & $1,2,3,5$-Tetrachlorobenzene & 4.67 & & 3360 & [13] \\
\hline & $\begin{array}{l}\text { rainbow trout (Salmo } \\
\text { gairdneri) }\end{array}$ & $1.8 \pm 0.9$ & $0.1-0.6$ & Pentachlorobenzene & 5.2 & & 4080 & [13] \\
\hline & Guppy (Poecilia reticulata) & $0.8 \pm 0.2$ & $0.1-0.4$ & 1,4-Diiodobenzene & 3.44 & & 2880 & {$[13]$} \\
\hline & Guppy (Poecilia reticulata) & $2.8 \pm 1.1$ & $0.1-0.4$ & Pentachlorobenzene & 5.2 & & 2352 & [13] \\
\hline & Medaka (Oryzias latipes) & 5.0 & $0.2-0.5$ & Aniline & 1.08 & & $249.6( \pm 21.6)$ & [14] \\
\hline & Medaka (Oryzias latipes) & 5.0 & $0.2-0.5$ & 4-Chloroaniline & 1.88 & & $688.8( \pm 64.8)$ & [14] \\
\hline & $\begin{array}{l}\text { mosquito fish (Gambusia } \\
\text { affinis) }\end{array}$ & 3.1 & 1.9 & 1,4-Dichlorobenzene & 3.44 & $112( \pm 20)$ & $3500( \pm 400)$ & {$[15]$} \\
\hline & $\begin{array}{l}\text { mosquito fish (Gambusia } \\
\text { affinis) }\end{array}$ & 3.1 & 1.9 & 1,4-Dibromobenzene & 3.89 & $272( \pm 67)$ & $10400( \pm 2900)$ & {$[15]$} \\
\hline & $\begin{array}{l}\text { mosquito fish (Gambusia } \\
\text { affinis) }\end{array}$ & 3.1 & 1.9 & 1,2,3-Trichlorobenzene & 4.07 & $470( \pm 10)$ & $10700( \pm 2100)$ & {$[15]$} \\
\hline & $\begin{array}{l}\text { mosquito fish (Gambusia } \\
\text { affinis) }\end{array}$ & 3.1 & 1.9 & $1,2,3,5$-Tetrachlorobenzene & 4.67 & $631( \pm 96)$ & $20000( \pm 7000)$ & [15] \\
\hline & $\begin{array}{l}\text { mosquito fish (Gambusia } \\
\text { affinis) }\end{array}$ & 3.1 & 1.9 & 1,2,4-Tribromobenzene & 4.98 & $1040( \pm 320)$ & $26200( \pm 8200)$ & {$[15]$} \\
\hline & $\begin{array}{l}\text { mosquito fish (Gambusia } \\
\text { affinis) }\end{array}$ & 3.1 & 1.9 & Pentachlorobenzene & 5.2 & $1520( \pm 600)$ & $34000( \pm 12000)$ & {$[15]$} \\
\hline & $\begin{array}{l}\text { mosquito fish (Gambusia } \\
\text { affinis) }\end{array}$ & 3.1 & 1.9 & Hexachlorobenzene & 5.77 & $1850( \pm 700)$ & $47000( \pm 18000)$ & [15] \\
\hline & $\begin{array}{l}\text { mosquito fish (Gambusia } \\
\text { affinis) }\end{array}$ & 3.1 & 1.9 & $1,2,4,5$-Tetrsbromobenzene & 6.04 & $900( \pm 270)$ & $23700( \pm 3800)$ & [15] \\
\hline & Guppy (Poecilia reticulata) & $10.5 \pm 1.9$ & 0.456 & 4-Chloroaniline & 1.88 & 110.4 & 1708.8 & [16] \\
\hline & Guppy (Poecilia reticulata) & $15 \pm 3$ & 0.349 & 1,2,3,5-Tetrachlorobenzene & 4.66 & 1608 & $10720^{\mathrm{b}}$ & {$[17]$} \\
\hline & guppy (Poecilia reticulata) & $15 \pm 3$ & 0.349 & 2,3,4,5-Tetrachloroaniline & 4.57 & 816 & $5440^{\mathrm{b}}$ & {$[17]$} \\
\hline & guppy(Poecilia reticulata) & $15 \pm 3$ & 0.349 & 2,3,5,6-Tetrachloroaniline & 4.46 & 1272 & $8480^{\mathrm{b}}$ & {$[17]$} \\
\hline & guppy(Poecilia reticulata) & $15 \pm 3$ & 0.349 & Pentachloroaniline & 5.08 & 1632 & $10880^{\mathrm{b}}$ & {$[17]$} \\
\hline & zebrafish $($ B. rerio $)$ & n.a. & 0.36 & 3,4-Dichloroaniline & 2.69 & & 1312.8 & {$[18]$} \\
\hline & zebrafish (B. rerio) & n.a. & 0.36 & Phenol & 1.46 & & 21.84 & {$[18]$} \\
\hline & zebrafish (B. rerio) & n.a. & 0.36 & Lindane & 3.72 & & 895.2 & {$[18]$} \\
\hline & $\begin{array}{l}\text { rainbow trout } \\
\text { (Oncorhynchus mykiss) }\end{array}$ & n.a. & 0.001045 & 3,4-Dichloroaniline & 2.69 & & 384 & [19] \\
\hline & $\begin{array}{l}\text { Golden ide } \\
\text { (Leuciscus idus melanotus) }\end{array}$ & n.a. & 0.00146 & 3,4-Dichloroaniline & 2.69 & & 163.2 & [19] \\
\hline & zebrafish (B. rerio) & n.a & $0.15-0.45$ & Aniline & 1.08 & & 266.4 & [20] \\
\hline & zebrafish (B. rerio) & n.a & $0.15-0.45$ & 2-Chloroaniline & 1.9 & & 170.4 & [20] \\
\hline & zebrafish (B. rerio) & n.a & $0.15-0.45$ & 3-Chloroanilne & 1.88 & & 458.4 & [20] \\
\hline & zebrafish (B. rerio) & n.a & $0.15-0.45$ & 3-Chloroaniline & 1.83 & & 1029.6 & {$[20]$} \\
\hline & zebrafish (B. rerio) & n.a & $0.15-0.45$ & 2,4-Dichloroaniline & 2.78 & & 345.6 & [20] \\
\hline & zebrafish (B. rerio) & n.a & $0.15-0.45$ & 3.4-Dichloroaniline & 2.69 & & 1884 & [20] \\
\hline & zebrafish (B. rerio) & n.a & $0.15-0.45$ & 2-Nitroaniline & 1.85 & & 643.2 & [20] \\
\hline & zebrafish (B. rerio) & n.a & $0.15-0.45$ & 3-Nitroaniline & 1.37 & & 141.6 & {$[20]$} \\
\hline & zebrafish (B. rerio) & n.a & $0.15-0.45$ & 4-Nitroaniline & 1.39 & & 309.6 & [20] \\
\hline & zebrafish $($ B. rerio $)$ & n.a & $0.15-0.45$ & 2,4-Dinitroaniline & 1.84 & & 868.8 & [20] \\
\hline & guppy(Poecilia reticulata) & $5.4 \pm 2.0$ & 0.62 & 1,4-Dichlorobenzene & 3.53 & & 1800 & [21] \\
\hline & guppy(Poecilia reticulata) & $5.4 \pm 2.0$ & 0.62 & 1,2,3-Trichlorobenzene & 4.2 & & 8299.2 & {$[21]$} \\
\hline & guppy(Poecilia reticulata) & $5.4 \pm 2.0$ & 0.62 & 1,3,5-Trichlorobenzene & 4.2 & & 7999.2 & [21] \\
\hline & guppy(Poecilia reticulata) & $5.4 \pm 2.0$ & 0.62 & $1,2,3,5$-Tetrachlorobenzene & 4.94 & & 15000 & [21] \\
\hline & guppy(Poecilia reticulata) & $5.4 \pm 2.0$ & 0.62 & Pentachlorobenzene & 5.69 & & 22000.8 & {$[21]$} \\
\hline & guppy(Poecilia reticulata) & $5.4 \pm 2.0$ & 0.62 & Hexchlorobenzene & 6.44 & & 10000.8 & [21] \\
\hline & Carp (Cyprinus Carpio $)$ & 9.8 & $5.0-12$ & 2,4-Dinitrotoluene & 1.98 & & 240 & [22] \\
\hline & fathead minnow ( $P$. promelas) & 23.8 & 0.045 & $1,2,3$-Trichlorobenzene & 6.1 & 700 & $2941^{\mathrm{b}}$ & [23] \\
\hline & fathead minnow (P. promelas) & 23.8 & 0.045 & 1,2,3,4-Tetrachlorobenzene & 4.635 & 1400 & $5882^{\mathrm{b}}$ & [23] \\
\hline & fathead minnow ( $P$. promelas $)$ & 23.8 & 0.045 & Pentachlorobenzene & 5.183 & 2200 & $9244^{\mathrm{b}}$ & [23] \\
\hline & fathead minnow ( $P$. promelas $)$ & 9.96 & 0.068 & 1,2,3-Trichlrobenzene & 6.1 & 1000 & $10040^{\mathrm{b}}$ & [23] \\
\hline & fathead minnow (P. promelas) & 9.96 & 0.068 & $1,2,3,4$-Tetrachlorobenzene & 4.635 & 2000 & $20080^{\mathrm{b}}$ & {$[23]$} \\
\hline & fathead minnow (P. promelas) & 9.96 & 0.068 & Pentachlorobenzene & 5.183 & 3000 & $30120^{\mathrm{b}}$ & [23] \\
\hline & fathead minnow ( $P$. promelas $)$ & 9.16 & 0.22 & 1,2,3-Trichlorobenzene & 6.1 & 1300 & $14192^{\mathrm{b}}$ & [23] \\
\hline & fathead minnow ( $P$. promelas $)$ & 9.16 & 0.22 & $1,2,3,4$-Tetrachlorobenzene & 4.635 & 1800 & $19651^{\mathrm{b}}$ & [23] \\
\hline & fathead minnow (P. promelas) & 9.16 & 0.22 & Pentachlorobenzene & 5.183 & 1800 & $19651^{\mathrm{b}}$ & [23] \\
\hline & fathead minnow ( $P$. promelas $)$ & 9.16 & 0.22 & Hexachlorobenzene & 6.9 & 5900 & $64410^{\mathrm{b}}$ & [23] \\
\hline & fathead minnow (P. promelas) & 7.41 & 0.41 & $1,2,3$-Trichlorobenzene & 6.1 & 800 & $10796^{\mathrm{b}}$ & {$[23]$} \\
\hline & fathead minnow (P. promelas) & 7.41 & 0.41 & 1,2,3,4-Tetrachlorobenzene & 4.635 & 1200 & $16194^{\mathrm{b}}$ & [23] \\
\hline & fathead minnow ( $P$. promelas $)$ & 7.41 & 0.41 & Pentachlorobenzene & 5.183 & 1300 & $17544^{\mathrm{b}}$ & [23] \\
\hline & fathead minnow ( $P$. promelas) & 7.41 & 0.41 & Hexachlorobenzene & 6.9 & 6300 & $85020^{\mathrm{b}}$ & [23] \\
\hline & fathead minnow (P. promelas) & 1.47 & 1.17 & 1,2,3-Trichlorobenzene & 6.1 & 800 & $54422^{\mathrm{b}}$ & [23] \\
\hline & fathead minnow (P. promelas) & 1.47 & 1.17 & $1,2,3,4$-Tetrachlorobenzene & 4.635 & 1200 & $81633^{\mathrm{b}}$ & [23] \\
\hline & fathead minnow ( $P$. promelas $)$ & 1.47 & 1.17 & Pentachlorobenzene & 5.183 & 1500 & $102041^{\mathrm{b}}$ & [23] \\
\hline & fathead minnow (P. promelas) & 1.47 & 1.17 & Hexachlorobenzene & 6.9 & 1100 & $74830^{\mathrm{b}}$ & {$[23]$} \\
\hline & fathead minnow ( $P$. promelas $)$ & 3.58 & 0.67 & 1,2,3-Trichlorobenzene & 6.1 & 1300 & $36313^{\mathrm{b}}$ & [23] \\
\hline & fathead minnow ( $P$. promelas $)$ & 3.58 & 0.67 & 1,2,3,4-Tetrachlorobenzene & 4.635 & 2900 & $81006^{\mathrm{b}}$ & [23] \\
\hline & fathead minnow (P. promelas) & 3.58 & 0.67 & Pentachlorobenzene & 5.183 & 3500 & $97765^{\mathrm{b}}$ & {$[23]$} \\
\hline & fathead minnow (P. promelas) & 3.58 & 0.67 & Hexachlorobenzene & 6.9 & 3400 & $94972^{\mathrm{b}}$ & [23] \\
\hline & fathead minnow ( $P$. promelas $)$ & 23.8 & 0.045 & Trichlorobenzene & 6.1 & 5900 & $24790^{\mathrm{b}}$ & [23] \\
\hline & fathead minnow (P. promelas) & 9.96 & 0.068 & Trichlorobenzene & 6.1 & 18000 & $180723^{\mathrm{b}}$ & {$[23]$} \\
\hline
\end{tabular}


(Table S3. continued)

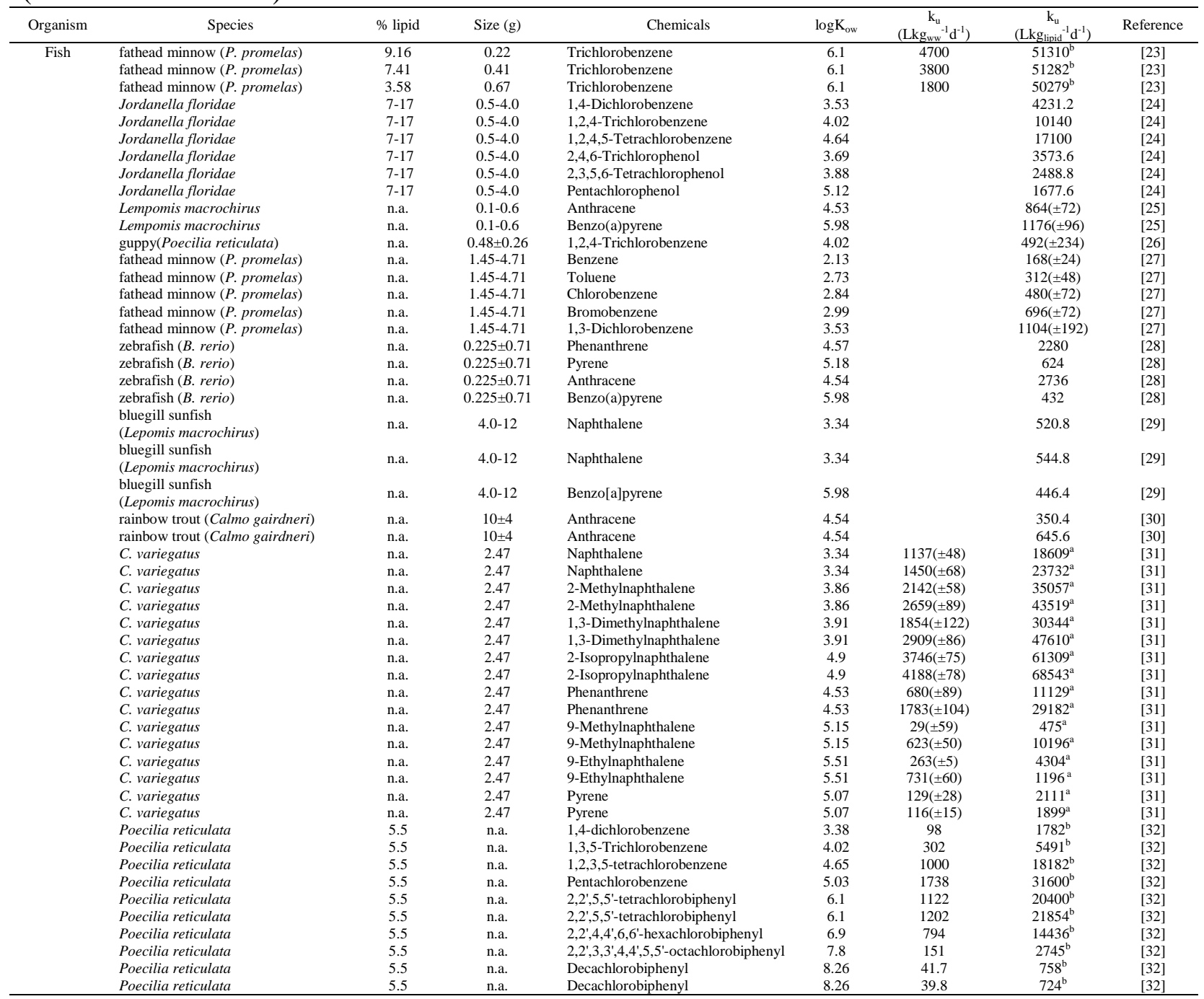

\footnotetext{
${ }^{\mathrm{a}}$ The values are calculated using average lipid content from Table S4.

${ }^{b}$ The values are calculated using lipid content in each literature.
} 
Table S4. Lipid contents for various aquatic organisms reported in the literature on wet weight basis.

\begin{tabular}{|c|c|c|c|}
\hline Organism & Species & Lipid content $(\%)$ & References \\
\hline \multirow[t]{18}{*}{ Algae* } & Scenedesmus obliquus & $2.4-2.8$ & [33] \\
\hline & Scenedesmusquadricauda & 0.38 & [33] \\
\hline & Scenedesmusdimorphus & $3.2-8$ & [33] \\
\hline & Chlamydomonas rheinhardii & 4.2 & [33] \\
\hline & Chlorella vulgaris & $2.8-4.4$ & [33] \\
\hline & Chlorella pyrenoidosa & 0.4 & [33] \\
\hline & Spirogyra sp. & $2.2-4.2$ & [33] \\
\hline & Dunaliella bioculata & 1.6 & [33] \\
\hline & Dunaliella salina & 1.2 & [33] \\
\hline & Euglena gracilis & $2.8-4$ & [33] \\
\hline & Prymnesium parvum & $4.4-7.6$ & [33] \\
\hline & Tetraselmis maculata & 0.6 & [33] \\
\hline & Porphyridium cruentum & $1.8-2.8$ & [33] \\
\hline & Spirulina platensis & $0.8-1.8$ & [33] \\
\hline & Spirulina maxima & $1.2-1.4$ & [33] \\
\hline & Synechoccus sp. & 2.2 & [33] \\
\hline & Anabaena cylindrica & $0.8-1.4$ & [33] \\
\hline & Average $( \pm$ S.D. $)$ & $2.4( \pm 1.72)$ & \\
\hline \multirow[t]{3}{*}{ Invertebrate } & Daphnia pulex & $1-3.5$ & [34] \\
\hline & Daphnia magna & 1.6 & [35] \\
\hline & Average ( \pm S.D. $)$ & $1.9( \pm 0.46)$ & \\
\hline \multirow[t]{32}{*}{ Fish } & guppy male & 2.2 & [36] \\
\hline & guppy female & 3.9 & [36] \\
\hline & goldfish & 3.3 & [36] \\
\hline & salmon & 4.6 & [36] \\
\hline & rainbow trout & 8.5 & [36] \\
\hline & fathead minnow & 3.6 & [36] \\
\hline & rainbow trout & $10.0 \pm 2.1$ & [7] \\
\hline & rainbow trout (Salmo gairdneri) & $6.84 \pm 0.93$ & [8] \\
\hline & turbot (Scophthalmus maximus) & 3 & [9] \\
\hline & zebrafish (Brachydanio rerio) & 2.92 & [10] \\
\hline & zebrafish (Brachydanio rerio) & $6.62 \pm 2.31$ & [11] \\
\hline & goldfish (Carassius auratus) & 6.3 & [12] \\
\hline & bluegill sunfish (Lepomis macrochirus) & $1.5 \pm 0.2$ & [13] \\
\hline & rainbow trout (Salmo gairdneri) & 2.9 & [13] \\
\hline & rainbow trout (Salmo gairdneri) & $1.8 \pm 0.9$ & [13] \\
\hline & guppy (Poecilia reticulata) & $0.8 \pm 0.2$ & [13] \\
\hline & guppy (Poecilia reticulata) & $2.8 \pm 1.1$ & [13] \\
\hline & medaka (Oryzias latipes) & 5 & [14] \\
\hline & mosquito fish (Gambusia affinis) & 3.1 & [15] \\
\hline & guppy (Poecilia reticulata) & $10.5 \pm 1.9$ & [16] \\
\hline & guppy (Poecilia reticulata) & $15 \pm 3$ & [17] \\
\hline & guppy (Poecilia reticulata) & $5.4 \pm 2.0$ & [21] \\
\hline & carp (Cyprinus Carpio) & 9.78 & [22] \\
\hline & fathead minnow (Pimephales promelas) & 23.8 & [23] \\
\hline & fathead minnow (Pimephales promelas) & 9.96 & [23] \\
\hline & fathead minnow (Pimephales promelas) & 9.16 & [23] \\
\hline & fathead minnow (Pimephales promelas) & 7.41 & [23] \\
\hline & fathead minnow (Pimephales promelas) & 1.47 & [23] \\
\hline & fathead minnow (Pimephales promelas) & 3.58 & [23] \\
\hline & flagflish (Jordanella floridae) & $7 \sim 17$ & [24] \\
\hline & guppy (Poecilia reticulata) & 5.5 & {$[32]$} \\
\hline & Average ( \pm S.D.) & $6.11( \pm 4.77)$ & \\
\hline
\end{tabular}

*Lipid contents were calculated from the reported lipid contents on dry-weight basis assuming that water content is 0.8 for all species. 
Table S5. Summary of reported toxicity test results using algae

\begin{tabular}{|c|c|c|c|c|c|c|c|c|c|}
\hline Chemicals name & CAS Reg. no & $\begin{array}{c}\text { Molecular } \\
\text { weight } \\
\left(\mathrm{g} \mathrm{mol}^{-1}\right)\end{array}$ & $\log \mathrm{K}_{\mathrm{ow}}$ & Solubility $\left(\mathrm{mg} \mathrm{L}^{-1}\right)$ & Species & $\begin{array}{l}\text { Duration } \\
\text { (h) }\end{array}$ & $\begin{array}{c}\text { Measured } \\
\mathrm{EC}_{50} \\
\left(\mathrm{mg} \mathrm{L}^{-1}\right)\end{array}$ & $\begin{array}{c}\text { Nominal } \\
\mathrm{EC}_{50} \\
\left(\mathrm{mg} \mathrm{L}^{-1}\right) \\
\end{array}$ & Classification \\
\hline & $\begin{array}{l}1328-53-6 \\
3590-84-9\end{array}$ & $\begin{array}{c}1127.19 \\
571.61\end{array}$ & $\begin{array}{l}17.40 \\
17.20\end{array}$ & $\begin{array}{c}7.00 \mathrm{E}-18-2.00 \mathrm{E}-16 \\
1.30 \mathrm{E}-12\end{array}$ & $\begin{array}{l}\text { Desmodesmus subspicatus } \\
\text { Scenedesmus subspicatus }\end{array}$ & $\begin{array}{l}72 \\
72\end{array}$ & & >solubility & $\begin{array}{ll}3 \\
3\end{array}$ \\
\hline $\begin{array}{l}\text { Stannane, tetraoctyl- } \\
\text { Di-n-n }\end{array}$ & $3590-84-9$ & 371.61 & 17.20 & $1.30 \mathrm{E}-12$ & $\begin{array}{l}\text { Scenedesmus subspicatus } \\
\text { Scenedesmus subspicatus }\end{array}$ & $\begin{array}{l}72 \\
72\end{array}$ & $\begin{array}{c}>0.21 \\
0.17\end{array}$ & & 3 \\
\hline Di-n-octyltin bis(ethylhexylthioglycolate) & $15571-58-1$ & 751.80 & 15.35 & $<1.00 \mathrm{E}+00$ & Scenedesmus subspicatus & 72 & $>0.06$ & & 2 \\
\hline Diisooctyl 2,2'-[(dioctylstannylene)bis(thio)]diacetate & 26401-97-8 & 751.80 & 15.35 & $<1.00 \mathrm{E}+00$ & $\begin{array}{l}\text { Scenedesmus subspicatus } \\
\text { Scenedesmus subspicatus }\end{array}$ & $\begin{array}{l}72 \\
72\end{array}$ & $\begin{array}{l}0.17 \\
>0.06\end{array}$ & & 2 \\
\hline Triisooctyl 2,2',2"-[(octylstannylidyne)tris(thio)]triacetate & $26401-86-5$ & 841.90 & 14.40 & $5.00 \mathrm{E}-01-2.70 \mathrm{E}+00$ & $\begin{array}{l}\text { Pseudokirchneriella subspicatus } \\
\text { Pseudokirchneriella subspicatus }\end{array}$ & $\begin{array}{l}72 \\
72\end{array}$ & $>0.445$ & & 1 \\
\hline $\begin{array}{l}\text { 2-Ethylhexyl 10-ethyl-4-[[2-[(2-ethylhexyl)oxy]-2-oxoethyl]thio]-4-octyl-7- } \\
\text { oxo-8-oxa-3,5-dithia-4-stannatetradecanoate }\end{array}$ & 27107-89-7 & 841.90 & 14.10 & $5.00 \mathrm{E}-01-2.70 \mathrm{E}+00$ & $\begin{array}{l}\text { Pseudokirchneriella subspicatus } \\
\text { Pseudokichneriella subspicatus }\end{array}$ & $\begin{array}{l}72 \\
72\end{array}$ & & $\begin{array}{l}>0.445 \\
0.03-0.3\end{array}$ & 1 \\
\hline Octadecanamide, N,N'-1,2-ethanediylbis- & $110-30-5$ & 593.04 & 13.98 & $1.10 \mathrm{E}-10$ & & & & & 4 \\
\hline Ditridecyl phthalate & $119-06-2$ & 530.84 & 13.45 & $1.48 \mathrm{E}-09$ & & & & & 4 \\
\hline Octadecyl 3-(3,5-di-tert-butyl-4-hydroxyphenyl)propionate & 2082-79-3 & 530.88 & 13.40 & 2.85E-02 & Scenedesmus subspicatus & 72 & >solubility & & 3 \\
\hline Monobutyltin tris (2-ethylhexylmercaptoacetate) & 26864-37-9 & 785.79 & 12.45 & $6.00 \mathrm{E}-02-4.40 \mathrm{E}+00$ & Scenedesmus subspicatus & 72 & $>0.36$ & & 3 \\
\hline Bis-(2-Ethylhexyl) nonanedioate & $103-24-2$ & 412.66 & 11.90 & $4.00 \mathrm{E}-01$ & Pseudokirchneriella subcapitata & 72 & & >solubility & 3 \\
\hline Di-Undecyl phthalate & $3648-20-2$ & 474.00 & 11.49 & $1.61 \mathrm{E}-07$ & non toxic & & & & 3 \\
\hline $\begin{array}{l}\text { 8-Oxa-3,5-dithia-4-stannatetradecanoic acid, 4,4-dibutyl-10-ethyl-7-oxo-, 2- } \\
\text { ethylhexyl ester }\end{array}$ & 10584-98-2 & 639.59 & 11.43 & $3.20 \mathrm{E}-01$ & $\begin{array}{l}\text { Scenedesmus subspicatus } \\
\text { Scenedesmus subspicatus }\end{array}$ & $\begin{array}{l}72 \\
72\end{array}$ & $\begin{array}{l}0.65 \\
0.56\end{array}$ & & 2 \\
\hline $\begin{array}{l}\text { 8-Oxa-3,5-dithia-4-stannatetradecanoic acid, 10-ethyl-4-[[2-[(2- } \\
\text { ethylhexyl)oxy]-2-oxoethyl]thio]-4-methyl-7-oxo-, 2-ethylhexyl ester }\end{array}$ & $57583-34-3$ & 743.71 & 10.98 & $1.80 \mathrm{E}+00-6.00 \mathrm{E}+00$ & non toxic & & & & 3 \\
\hline Methacrylic acid, 2-methyl-, eicosyl ester & 45294-18-6 & 366.63 & 10.61 & $5.01 \mathrm{E}-06$ & & & & & 4 \\
\hline Di(isodecyl) phthalate & $26761-40-0$ & 446.68 & 10.36 & $1.04 \mathrm{E}-05$ & Scenedesmus subspicatus & 72 & >solubility & & 3 \\
\hline 1,2-benzenedicarboxylic acid, di-2-propylheptyl ester & $53306-54-0$ & 446.68 & 10.36 & $2.24 \mathrm{E}-06$ & non toxic & & & & 3 \\
\hline 2-Butanone, $\mathrm{O}, \mathrm{O}^{\prime}, \mathrm{O}^{\prime \prime}$-(ethenylsilylidyne)trioxime & 2224-33-1 & 313.48 & 10.19 & 3.00E-05 & & & & & 4 \\
\hline Docosanoic acid & $112-85-6$ & 340.60 & 9.91 & $1.60 \mathrm{E}-02$ & Selenastrum capricornutum & 72 & & $>5.00$ & 3 \\
\hline 2-Butanone, $\mathrm{O}, \mathrm{O}^{\prime}, \mathrm{O}^{\prime \prime}$-(methylsilylidyne)trioxime & 22984-54-9 & 301.46 & 9.83 & $6.00 \mathrm{E}-05$ & Pseudokirchneriella subcapitata & 72 & & 94.00 & 2 \\
\hline 1-Docosanol & $661-19-8$ & 326.61 & 9.68 & $2.70 \mathrm{E}-03$ & & 12 & & & 4 \\
\hline Octadecyl methacrylate & $32360-05-7$ & 338.58 & 9.62 & $5.49 \mathrm{E}-05$ & & & & & 4 \\
\hline Stannane, tetrabutyl- & $1461-25-2$ & 347.18 & 9.37 & $<1.00 \mathrm{E}-01$ & Skeletonema costatum & 72 & & 0.05 & 1 \\
\hline Di(isononyl) phthalate & 28553-12-0 & 418.62 & 9.37 & $2.32 \mathrm{E}-05$ & non toxic & & & & 3 \\
\hline Stannane, dioctyloxo- & $870-08-6$ & 361.16 & 9.26 & $6.57 \mathrm{E}-05$ & & & & & 4 \\
\hline 1-Octadecene & $112-88-9$ & 252.49 & 9.04 & $1.51 \mathrm{E}-04$ & Selenastrum capricornutum & 72 & & >solubility & 3 \\
\hline Cyclohexasiloxane, dodecamethyl- & $540-97-6$ & 444.39 & 8.82 & $5.13 \mathrm{E}-03$ & & & & & 4 \\
\hline 1-Eicosanol & $629-96-9$ & 298.56 & 8.70 & $2.70 \mathrm{E}-03$ & & & & & 4 \\
\hline Hexadecyl methacrylate & $2495-27-4$ & 310.52 & 8.64 & 4.44E-04 & & & & & 4 \\
\hline Dimethylin bis[2-ethylhexylthioglycolate] & 57583-35-4 & 555.42 & 8.48 & $1.00 \mathrm{E}-01-4.90 \mathrm{E}+00$ & Selenastrum capricornutum & 72 & & 270.00 & 2 \\
\hline \multirow[t]{2}{*}{ Bis(2-ethylhexyl) adipate } & \multirow[t]{2}{*}{$103-23-1$} & \multirow[t]{2}{*}{370.64} & \multirow[t]{2}{*}{8.39} & \multirow[t]{2}{*}{$3.20 \mathrm{E}-03$} & Selenastrum capricornutum & 96 & & >solubility & 3 \\
\hline & & & & & Scenedesmus subspicatus & 72 & & >solubility & \\
\hline Terephthalic acid, bis(2-ethylhexyl) ester & $6422-86-2$ & 390.57 & 8.39 & $4.00 \mathrm{E}-04$ & Selenastrum capricornutum & 72 & & $>0.86$ & 3 \\
\hline 1-Octadecanol & $112-92-5$ & 270.50 & 8.22 & $1.10 \mathrm{E}-03$ & Scenedesmus subspicatus & 72 & & 240.00 & 2 \\
\hline Hexadecane & $544-76-3$ & 226.45 & 8.20 & $9.19 \mathrm{E}-04$ & non toxic & & & & 3 \\
\hline Methacrylic acid, pentadecyl ester & $6140-74-5$ & 296.50 & 8.15 & $1.41 \mathrm{E}-03$ & & & & & 4 \\
\hline Pigment Yellow 13 & $5102-83-0$ & 685.62 & 8.11 & $3.50 \mathrm{E}-04$ & & & & >solubility & 3 \\
\hline 1-Hexadecen-3-ol, 3,7,11,15-tetramethyl- & $505-32-8$ & 296.54 & 8.10 & $5.80 \mathrm{E}+00$ & Scenedesmus subspicatus & 72 & & >solubility & 3 \\
\hline 1-Hexadecene & $629-73-2$ & 224.43 & 8.06 & $1.44 \mathrm{E}-03$ & Selenastrum capricornutum & 72 & & $\overrightarrow{\text { solubility }}$ & 3 \\
\hline 1-Dodecene & $112-41-4$ & 168.33 & & $1.13 \mathrm{E}-01$ & Selenastrum capricornutum & 96 & & $\begin{array}{l}\text { somomity } \\
22.00\end{array}$ & 2 \\
\hline Trisiloxane, 1,1,1,3,5,5,5-heptamethyl- & $1873-88-7$ & 222.51 & 7.84 & $2.00 \mathrm{E}-02$ & Pseudokirchneriella subcapitata & 72 & $>0.019$ & & 3 \\
\hline Trisiloxane, 1,1,1,5,5,5-hexamethyl-3,3-bis[(trimethylsilyl)oxy]- (M4Q) & $3555-47-3$ & 384.85 & 7.80 & $1.50 \mathrm{E}-04$ & & & & & 4 \\
\hline Cyclododecane, 1,2,5,6,9,10-hexabromo- & $3194-55-6$ & 641.70 & 7.74 & $3.40 \mathrm{E}-03$ & Scenedesmus subspicatus & 72 & & 0.05 & 2 \\
\hline Pentadecane & $629-62-9$ & 212.42 & 7.71 & $2.87 \mathrm{E}-03$ & non toxic & & & & 3 \\
\hline Tetradecyl methacrylate & $2549-53-3$ & 282.47 & 7.66 & 4.46E- 03 & & & & & 4 \\
\hline Octadecanamine, $\mathrm{N}, \mathrm{N}$-dimethyl-, $\mathrm{N}$-oxide & $2571-88-2$ & 313.57 & 7.62 & $3.18 \mathrm{E}-03$ & Selenastrum capricornutum & 72 & & 0.14 & 2 \\
\hline
\end{tabular}




\section{(Table S5. continued)}

\begin{tabular}{|c|c|c|c|c|c|c|c|c|c|}
\hline 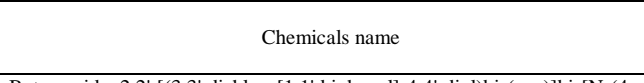 & CAS Reg. no & $\begin{array}{c}\text { Molecular } \\
\text { weight } \\
\left(\mathrm{g} \mathrm{mol}^{-1}\right) \\
\end{array}$ & $\log \mathrm{K}_{\mathrm{ow}}$ & Solubility (mg L $\left.{ }^{-1}\right)$ & Species & $\begin{array}{l}\text { Duration } \\
\text { (h) }\end{array}$ & $\begin{array}{c}\text { Measured } \\
\mathrm{ES}_{50} \\
\left(\mathrm{mg} \mathrm{L}^{-1}\right) \\
\end{array}$ & $\begin{array}{c}\text { Nominal } \\
\text { EC } \\
\left(\mathrm{ES} \mathrm{L}_{5}^{-1}\right) \\
\left(\mathrm{mg} \mathrm{L}^{-1}\right.\end{array}$ & Classification \\
\hline $\begin{array}{l}\text { Butanamide, 2,2'-[(3,3'-dichloro[1,1'-biphenyl]-4,4'-diyl)bis(azo)]bis[N-(4- } \\
\text { chloro-2,5-dimethoxyphenyl)-3-oxo- }\end{array}$ & $5567-15-7$ & 818.50 & 7.54 & $8.10 \mathrm{E}-03$ & \multirow[t]{2}{*}{ non toxic } & & & & 3 \\
\hline $\begin{array}{l}\text { 9-Octadecen-1-ol, (9Z)- } \\
\text { Phenol, dodecyl- }\end{array}$ & $\begin{array}{c}143-28-2 \\
27193-86-8\end{array}$ & $\begin{array}{l}268.49 \\
262.44\end{array}$ & $\begin{array}{l}7.50 \\
7.46\end{array}$ & 4.20E-02 & & & & & $\begin{array}{l}4 \\
4\end{array}$ \\
\hline Benzene, undecyl- & $6742-54-7$ & 232.41 & 7.45 & $4.10 \mathrm{E}-02$ & \multirow{3}{*}{$\begin{array}{l}\text { Chironomus tentans } \\
\text { Selenastrum capricornutum } \\
\text { non toxic }\end{array}$} & \multirow[t]{3}{*}{$\begin{array}{l}96 \\
96\end{array}$} & & \multirow[t]{3}{*}{$\begin{array}{l}\text { > water sol } \\
>\text { water sol }\end{array}$} & 3 \\
\hline Tetradecane & $629-59-4$ & 198.40 & 7.20 & $9.19 \mathrm{E}-03$ & & & & & 3 \\
\hline Tridecyl 2-methacrylate & $2495-25-2$ & 268.44 & 7.17 & & & & & & 4 \\
\hline $\begin{array}{l}\text { Phenol, tetrapropylene } \\
\text { Pats }\end{array}$ & $57427-55-1$ & & 7.14 & & Pseudokirchneriella subspicatus & 72 & 0.09 & & 1 \\
\hline 1-Tetradecene & $1120-36-1$ & 196.38 & 7.08 & 4.00E-04 & Selenastrum capricornutum & 96 & 0.09 & $=>1000$ & 3 \\
\hline C.I. Pigment Yellow 12 & $6358-85-6$ & 629.51 & 7.05 & 4.00E-04 & Selenastrum capricornutum & 72 & & $\begin{array}{c}>\text { water } \\
\text { solubility }\end{array}$ & 3 \\
\hline Bis(2-ethylhexyl) phthalate & $117-81-7$ & 390.57 & 7.00 & $1.30 \mathrm{E}+00$ & Scenedesmus subspicatus & 72 & & $>0.1$ & 3 \\
\hline Cyclotetrasiloxane, octamethyl- & $556-67-2$ & 296.62 & 6.79 & & & & & & 4 \\
\hline Tridecane & $629-50-5$ & 184.37 & 6.73 & & & & & & 4 \\
\hline 1-Hexadecanol & $36653-82-4$ & 242.45 & 6.73 & $1.30 \mathrm{E}-02$ & Scenedesmus subspicatus & 96 & & $>980$ & 3 \\
\hline Dodecyl methacrylate & $142-90-5$ & 254.42 & 6.68 & & & & & & 4 \\
\hline hexadecyldimethylamine $\mathrm{N}$-oxide & $7128-91-8$ & 285.52 & 6.64 & $3.20 \mathrm{E}-02$ & Selenastrum capricornutum & 72 & & 0.06 & 2 \\
\hline Trisiloxane, octamethyl- & $107-51-7$ & 236.54 & 6.60 & $3.40 \mathrm{E}-02$ & Pseudokirchneriella subcapitata & 72 & & 0.01 & 1 \\
\hline Methyl laurate & $111-82-0$ & 214.35 & 6.50 & $1.39 \mathrm{E}+00$ & Pseudokirchneriella subcapitata & 72 & 0.02 & & 2 \\
\hline Decanedioic acid, bis (2,2,6,6-tetramethyl-4-piperidinyl) ester & $52829-07-9$ & 480.74 & 6.50 & $1.88 \mathrm{E}+01$ & Scenedesmus subspicatus & 72 & & 1.90 & 1 \\
\hline Benzene, 1,1'-oxybis-, octabromo deriv. & $32536-52-0$ & 801.38 & 6.29 & $5.00 \mathrm{E}-04$ & & & & & 4 \\
\hline Benzene, 1,1'-oxybis[2,3,4,5,6-pentabromo]- & $1163-19-5$ & 959.17 & 6.27 & $<1.00 \mathrm{E}-05$ & $\begin{array}{l}\text { Skeletonema costatum } \\
\text { Thalassiosira pseudonana } \\
\text { Chlorella sp }\end{array}$ & $\begin{array}{l}72 \\
72 \\
96\end{array}$ & & $\begin{array}{l}\text { >solubility } \\
\text { >solubility } \\
\text { > solubility }\end{array}$ & 3 \\
\hline p-Cresol, 6,6'-di-tert-butyl-2,2'-methylenedi- & $119-47-1$ & 340.51 & 6.25 & $2.00 \mathrm{E}-02$ & Selenastrum capricornutum & 72 & & $>5.0$ & 3 \\
\hline 1-Pentadecanol & $629-76-5$ & 228.42 & 6.24 & $1.02 \mathrm{E}-01$ & & & & & 4 \\
\hline Dodecane & $112-40-3$ & 170.34 & 6.23 & $1.10 \mathrm{E}-01$ & & & & & 4 \\
\hline 1-Dodecanethiol & $112-55-0$ & 202.40 & 6.20 & $1.00 \mathrm{E}+00$ & Pseudokirchneriella subcapitata & 72 & & $<0.0145$ & 1 \\
\hline 1,1'-(1,1-dimethyl-3-methylene-1,3-propanediyl)bisbenzene & $6362-80-7$ & 236.36 & 6.20 & $2.30 \mathrm{E}-01$ & $\begin{array}{l}\text { Pseudokirchneriella subcapitata } \\
\text { Psula }\end{array}$ & 72 & $>0.059$ & 0.0143 & 3 \\
\hline $\begin{array}{l}\text { tert-Dodecanethiol } \\
\text { ticticle }\end{array}$ & $25103-58-6$ & 202.40 & 6.20 & $2.50 \mathrm{E}-01$ & 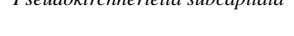 & 12 & 20.039 & & 4 \\
\hline Cyclohexane, 1,1'-methylenebis[4-isocyanato- & $5124-30-1$ & 262.35 & 6.11 & $1.21 \mathrm{E}-01$ & Scenedesmus subspicatus & 72 & & $>5$ & 3 \\
\hline Phenol, 2,6-bis(1,1-dimethylethyl)-4-(1-methylpropyl)- & $17540-75-9$ & 262.44 & 6.10 & $2.50 \mathrm{E}+00$ & 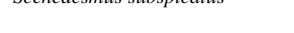 & & & 年 & 4 \\
\hline Dodecene & $25378-22-7$ & 168.33 & 6.10 & $1.25 \mathrm{E}-01$ & & & & & 4 \\
\hline Ethanol, 2,2'-(octadecyloxidoimino)bis- & $14048-77-2$ & 373.63 & 6.08 & $2.90 \mathrm{E}-02$ & & & & & 4 \\
\hline Phenol, nonyl- & 25154-52-3 & 220.36 & 5.99 & $1.57 \mathrm{E}+00$ & $\begin{array}{l}\text { Scenedesmus subspicatus } \\
\text { Scenedesmus subspicatus }\end{array}$ & $\begin{array}{l}72 \\
72\end{array}$ & & $\begin{array}{l}1.30 \\
0.32\end{array}$ & 1 \\
\hline 1,2,4-Benzenetricarboxylic acid, tris(2-ethylhexyl) ester & $3319-31-1$ & 546.79 & 5.94 & $1.30 \mathrm{E}-01$ & Selenastrum capricornutum & 72 & & $>100$ & 3 \\
\hline 2,2',6,6'-Tetrabromo-4,4'-isopropylidenediphenol & 79-94-7 & 543.90 & 5.90 & $1.26 \mathrm{E}+00$ & Pseudokirchneriella subcapitata & 72 & $0.09-0.89$ & & 1 \\
\hline Ethanol, 2,2'-[(9Z)-9-octadecenyloxidoimino $]$ bis- & $93962-62-0$ & 371.61 & 5.86 & $4.50 \mathrm{E}-02$ & Selenastrum & 72 & & 0.16 & 2 \\
\hline Triisobutylene & 7756-94-7 & 168.33 & 5.85 & $1.90 \mathrm{E}-01$ & & & & & 4 \\
\hline Stannane, dichlorodiocytyl- & $3542-36-7$ & 416.07 & 5.82 & $2.40 \mathrm{E}-01-2.80 \mathrm{E}-01$ & Scenedesmus subspicatus & 72 & $>0.002$ & & 3 \\
\hline Benzene, dodecyl- & $123-01-3$ & 246.44 & 5.75 & $4.10 \mathrm{E}-02$ & Selenastrum capricornutum & 96 & >solubility & & 3 \\
\hline 1-Tetradecanol & $112-72-1$ & 214.39 & 5.75 & $1.91 \mathrm{E}-01$ & non toxic & & & & 3 \\
\hline n-Undecane & $1120-21-4$ & 156.31 & 5.74 & 4.00E-03 & $\begin{array}{l}\text { Pseudokirchneriella } \\
\text { subcapitata]: }\end{array}$ & 72 & $>0.0059$ & & 3 \\
\hline Decene & 25339-53-1 & 140.27 & 5.70 & $2.10 \mathrm{E}-01$ & $\begin{array}{l}\text { subcaptatata: } \\
\text { green algae }\end{array}$ & 96 & 0.12 & & 1 \\
\hline Phosphine, triphenyl- & $603-35-0$ & 262.29 & 5.69 & $1.65 \mathrm{E}-01$ & Desmosdesmus subspicatus & 72 & & $>5$ & 3 \\
\hline 1-Tetradecanamine, N,N-dimethyl-, N-oxide & $3332-27-2$ & 257.46 & 5.66 & 3.20E-01 & Selenastrum capricornutum & 72 & & 0.08 & 1 \\
\hline 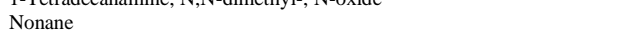 & $111-84-2$ & 128.26 & 5.65 & $4.06 \mathrm{E}-01$ & & & & & 4 \\
\hline Peroxide, bis(1-methyl-1-phenylethyl)- & $80-43-3$ & 270.37 & 5.60 & $4.30 \mathrm{E}-01$ & Pseudokirchneriella subcapitata & 72 & >solubility & & 3 \\
\hline Bumetrizole & $3896-11-5$ & 315.81 & 5.55 & $<1.00 \mathrm{E}+00$ & Desmodesmus subspicatus & 72 & solubility & & 3 \\
\hline Metilox & $6386-38-5$ & 292.42 & $5.50-$ & $2.20 \mathrm{E}+00$ & Scenedesmus subspicatus & 72 & 2.30 & & 1 \\
\hline 2-Ethylhexyl vinyl ether & $103-44-6$ & 156.27 & 5.50 & $1.80 \mathrm{E}+00$ & & & & & 4 \\
\hline $\begin{array}{l}\text { Peroxide, [1,3(or 1,4)-phenylenebis(1-methylethylidene)]bis[(1,1- } \\
\text { dimethylethyl) }\end{array}$ & $25155-25-3$ & 338.49 & 5.50 & 4.00E-02 & Pseudokirchneriella subcapitata & 72 & >solubility & & 3 \\
\hline
\end{tabular}


(Table S5. continued)

\begin{tabular}{|c|c|c|c|c|c|c|c|c|c|}
\hline Chemicals name & CAS Reg. no & $\begin{array}{c}\text { Molecular } \\
\text { weight } \\
\left(\mathrm{g} \mathrm{mol}^{-1}\right)\end{array}$ & $\log \mathrm{K}_{\mathrm{ow}}$ & Solubility $\left(\mathrm{mg} \mathrm{L}^{-1}\right)$ & Species & $\begin{array}{l}\text { Duration } \\
\text { (h) }\end{array}$ & $\begin{array}{c}\text { Measured } \\
\mathrm{EC}_{50} \\
\left(\mathrm{mg} \mathrm{L}^{-1}\right)\end{array}$ & $\begin{array}{c}\text { Nominal } \\
\mathrm{EC}_{50} \\
\left(\mathrm{mg} \mathrm{L}^{-1}\right) \\
\end{array}$ & Classification \\
\hline 1-Dodecanamine, $\mathrm{N}, \mathrm{N}$-dimethyl- & $112-18-5$ & 213.41 & 5.47 & $1.00 \mathrm{E}+01$ & $\begin{array}{l}\text { Scenedesmus subspicatus } \\
\text { Scenedesmus subspicatus } \\
\text { Scenedesmus subspicatus } \\
\text { Scenedesmus subspicatus }\end{array}$ & $\begin{array}{l}72 \\
72 \\
72 \\
72\end{array}$ & & $\begin{array}{l}0.02 \\
0.06 \\
0.09 \\
0.01\end{array}$ & 1 \\
\hline $\begin{array}{l}\text { 1,3,5-Triazine-2,4,6(1H,3H,5H)-trione, 1,3,5-tris[[3,5-bis(1,1- } \\
\text { dimethylethyl)-4-hydroxyphenyl]methyl]- }\end{array}$ & $27676-62-6$ & 784.10 & 5.45 & $1.00 \mathrm{E}+01$ & & & & & 4 \\
\hline Ethanone, 1-(5,6,7,8-tetrahydro-3,5,5,6,8,8-hexamethyl-2-naphthalenyl)- & $1506-02-1$ & 258.41 & 5.40 & $1.22 \mathrm{E}+00$ & $\begin{array}{l}\text { Pseudokirchneriella subcapitata } \\
\text { Pseudokirchneriella subcapitata }\end{array}$ & $\begin{array}{l}72 \\
72\end{array}$ & $>0.835$ & $>0.797$ & 3 \\
\hline Ethanone, 1-(5,6,7,8-tetrahydro-3,5,5,6,8,8-hexamethyl-2-naphthalenyl)- & 21145-77-7 & 258.41 & 5.40 & $1.22 \mathrm{E}+00$ & Pseudokirchneriella subcapitata & 72 & $>0.835$ & & 3 \\
\hline 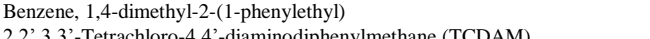 & $\begin{array}{l}6165-51-1 \\
4240-73-3\end{array}$ & $\begin{array}{l}210.31 \\
322.02\end{array}$ & 5.39 & & Selenastrum capricornutum & 72 & & $>1.54$ & 3 \\
\hline $\begin{array}{l}\text { 2,2',3,3'-Tetrachloro-4,4'-diaminodiphenylmethane (TCDAM) } \\
\text { Disiloxane, 1,3-diethenyl-1,1,3,3-tetramethyl- }\end{array}$ & $\begin{array}{c}42240-73-3 \\
2627-95-4\end{array}$ & $\begin{array}{l}322.02 \\
186.40\end{array}$ & $\begin{array}{l}5.39 \\
5.36\end{array}$ & $\begin{array}{l}2.27 \mathrm{E}-01-7.88 \mathrm{E}-01 \\
2.07 \mathrm{E}-01\end{array}$ & Pseudokirchnerella subcapitata & 72 & $>0.12$ & & $\begin{array}{l}4 \\
3\end{array}$ \\
\hline & $818-08-6$ & 248.94 & 5.33 & $6.73 \mathrm{E}-01$ & 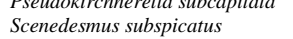 & 72 & 0.12 & 1.60 & 2 \\
\hline Cyclopenta[g]-2-benzopyran, 1,3,4,6,7,8-hexahydro-4,6,6,7,8,8-hexamethyl- & $1222-05-5$ & 258.41 & 5.30 & $1.75 \mathrm{E}+00$ & Pseudokirchneriella subcapitata & 72 & 0.85 & & 1 \\
\hline Isodecyl methacrylate & 29964-84-9 & 226.36 & 5.28 & & & & & & 4 \\
\hline $\begin{array}{l}\text { 1(3H)-Isobenzofuranone, 6-(dimethylamino)-3,3-bis[4- } \\
\text { (dimethylamino)phenyl]- }\end{array}$ & $1552-42-7$ & 415.54 & 5.27 & $1.00 \mathrm{E}+01$ & & & & & 4 \\
\hline 1-Tridecanol & $112-70-9$ & 200.37 & 5.26 & $3.80 \mathrm{E}-01$ & non toxic & & & & 3 \\
\hline Triphenylmethyl chloride & $76-83-5$ & 278.78 & 5.25 & $5.35 \mathrm{E}-01$ & & & & & 4 \\
\hline n-Decane & $124-18-5$ & 142.29 & 5.25 & & & & & & 4 \\
\hline Diphenylmethane diisocyanate (4,4'-M.D.I.) & $101-68-8$ & 250.25 & 5.22 & & non toxic & & & & 3 \\
\hline 2,2'-Diphenyl methane diisocyanate & 2536-05-2 & 250.26 & 5.22 & & & & & & 4 \\
\hline 2,4'-Diphenyl methane disocyanate & 5873-54-1 & 250.26 & 5.22 & & & & & & 4 \\
\hline Benzene, 1,1'-methylenebis(isocyanato- & $26447-40-5$ & 250.26 & 5.22 & $8.29 \mathrm{E}-01$ & non toxic & & & & 3 \\
\hline Disiloxane, hexamethyl- & $107-46-0$ & 162.38 & 5.20 & $9.30 \mathrm{E}-01$ & $\begin{array}{l}\text { Pseudokirchneriella subcapitata } \\
\text { Pseudokirchneriella subcapitata }\end{array}$ & $\begin{array}{l}70 \\
95\end{array}$ & $\begin{array}{l}>0.55 \\
>0.55\end{array}$ & & 3 \\
\hline 1-Isotridecanol & 27458-92-0 & 200.37 & 5.19 & $5.24 \mathrm{E}+00$ & algae & 72 & $1.6-19$ & & 1 \\
\hline Octane & $111-65-9$ & 114.23 & 5.18 & $1.15 \mathrm{E}+00$ & & & & & 4 \\
\hline Ligroine & $8032-32-4$ & 114.23 & 5.18 & & & & & & 4 \\
\hline 1-Decanol & $112-30-1$ & 158.29 & 5.13 & $3.95 \mathrm{E}+01$ & & & & & 4 \\
\hline 1-Dodecanol & $112-53-8$ & 186.34 & 5.13 & $1.90 \mathrm{E}+00$ & Senedesmus subspicatus & & & 0.97 & 1 \\
\hline 1-Decene & 872-05-9 & 140.27 & 5.12 & $1.15 \mathrm{E}-01$ & Selenastrum capricornutum & 96 & & 22.00 & 2 \\
\hline 2,6-Di-tert-butyl-p-cresol & $128-37-0$ & 220.36 & 5.10 & $1.10 \mathrm{E}+00$ & $\begin{array}{l}\text { Scenedesmus subspicatus } \\
\text { Selenastrum capricornutum }\end{array}$ & $\begin{array}{l}72 \\
96\end{array}$ & $>.4$ & >solubility & 3 \\
\hline $\begin{array}{l}\text { 2-Propenoic acid, 2-methyl-, (1R,2R,4R)-1,7,7-trimethylbicyclo[2.2.1] hept- } \\
\text { 2-yl ester, rel- }\end{array}$ & 7534-94-3 & 222.33 & 5.09 & $5.44 \mathrm{E}+00$ & Pseudokirchneriella subcapitata & 72 & & 2.28 & 1 \\
\hline Silane, dichlorodiphenyl- & $80-10-4$ & 253.20 & 5.06 & $2.80 \mathrm{E}+00$ & & & & & 4 \\
\hline Cyclopentadiene, hexachloro- & $77-47-4$ & 272.77 & 5.04 & $1.03 \mathrm{E}+00-1.25 \mathrm{E}+00$ & Scenedesmus subspicatus & 72 & & 0.24 & 1 \\
\hline Pentaerythritol tetrakis[3-(3,5-di-tert-butyl-4-hydroxypheny & $6683-19-8$ & 1177.67 & 5.00 & $1.00 \mathrm{E}-01$ & non toxic & 12 & & $0.2+$ & 3 \\
\hline Pentene, 2,4,4-trimethyl- & $25167-70-8$ & 112.22 & 5.00 & $1.80 \mathrm{E}+00$ & Selenastrum capricornutum & 72 & 1.67 & & 1 \\
\hline 2-Ethylhexyl methacrylate & 688-84-6 & 198.31 & 4.95 & $1.60 \mathrm{E}+00$ & Selenastrum capricornutum & 72 & & 7.68 & 2 \\
\hline 2-Benzothiazolesulfenamide, $\mathrm{N}$-cyclohexyl- & $95-33-0$ & 264.41 & 4.93 & $3.20 \mathrm{E}-01$ & Selenastrum capricornutum & & & $>0.15$ & 3 \\
\hline 5-tert-Butyl-2,4,6-trinitro-m-xylene (Musk xylene) & $81-15-2$ & 297.26 & 4.90 & $1.50 \mathrm{E}-01$ & Scenedesmus subspicatus & 72 & & $>=0.15$ & 3 \\
\hline Neodecanoic acid, ethenyl ester & $51000-52-3$ & 198.30 & 4.90 & $5.90 \mathrm{E}+00$ & & & & & 4 \\
\hline Butyl benzyl phthalate & 85-68-7 & 312.36 & 4.84 & & & & & & 4 \\
\hline Perfluorooctanoic Acid & $335-67-1$ & 414.07 & 4.81 & $9.50 \mathrm{E}+03$ & Pseudokirchneriella subcapitata & 72 & & $>400$ & 3 \\
\hline 2-Benzothiazolesulfenamide, N,N-dicyclohexyl- & $4979-32-2$ & 346.55 & 4.80 & $1.90 \mathrm{E}-03$ & Selenastrum capricornutum & 72 & & $>0.0118$ & 3 \\
\hline Benzenesulfonic acid, dodecyl- & $27176-87-0$ & 326.50 & 4.78 & $6.03 \mathrm{E}-01$ & 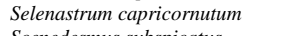 & 72 & 65.40 & & 2 \\
\hline Phenol, 5-chloro-2-(2,4-dichlorophenoxy)- & $3380-34-5$ & 289.54 & 4.76 & $1.00 \mathrm{E}+01$ & $\begin{array}{l}\text { Scenedesmus subspicatus } \\
\text { Pseudokirchneriella subcapitata }\end{array}$ & $\begin{array}{l}72 \\
72\end{array}$ & 0.00 & 0.00 & 1 \\
\hline Cyclohexane, 5-isocyanato-1-(isocyanatomethyl)-1,3,3-trimethyl- & $4098-71-9$ & 222.24 & 4.75 & $1.50 \mathrm{E}+01$ & $\begin{array}{l}\text { Desmodesmus subspicatus } \\
\text { Destate }\end{array}$ & 72 & & $>70$ & 3 \\
\hline p-Phenylenediamine, N-(1,3-dimethylbutyl)-N'-phenyl- & 793-24-8 & 268.50 & 4.68 & $1.00 \mathrm{E}+00$ & Selenastrum capricornutum & 96 & & 0.60 & 1 \\
\hline 1-Dodecanamine, $\mathrm{N}, \mathrm{N}$-dimethyl-, N-oxide & $1643-20-5$ & 229.41 & 4.67 & $3.13 \mathrm{E}+00$ & $\begin{array}{l}\text { Scenedesmus subspicatus } \\
\text { Selenastrum capricornutum }\end{array}$ & $\begin{array}{l}72 \\
72\end{array}$ & 0.11 & 0.13 & 1 \\
\hline $\begin{array}{l}\text { Amine oxides, cocoalkyldimethyl } \\
\text { Benzothiazole disulfide }\end{array}$ & $\begin{array}{c}61788-90-7 \\
120-78-5\end{array}$ & $\begin{array}{l}229.41 \\
332.47\end{array}$ & $\begin{array}{l}4.67 \\
4.66\end{array}$ & $3.13 \mathrm{E}+00$ & Selenastrum capricornutum & 72 & & 0.29 & 1 \\
\hline Heptane & $142-82-5$ & 100.21 & 4.66 & $3.55 \mathrm{E}+00$ & & & & & 4 \\
\hline 2-Naphthylisobutylether & $2173-57-1$ & 200.28 & 4.65 & $1.17 \mathrm{E}+00-6.91 \mathrm{E}+00$ & & & & & 4 \\
\hline Triphenyl phosphate & $115-86-6$ & 326.28 & 4.60 & $1.90 \mathrm{E}+00$ & Selenastrum capricornutum & 96 & & 2.00 & 1 \\
\hline Disperse Yellow-42 & $5124-25-4$ & & 4.60 & $2.00 \mathrm{E}-01$ & & & & & 4 \\
\hline
\end{tabular}


(Table S5. continued)

\begin{tabular}{|c|c|c|c|c|c|c|c|c|c|}
\hline Chemicals name & CAS Reg. no & $\begin{array}{c}\text { Molecular } \\
\text { weight } \\
\left(\mathrm{g} \mathrm{mol}^{-1}\right)\end{array}$ & $\log \mathrm{K}_{\mathrm{ow}}$ & Solubility $\left(\mathrm{mg} \mathrm{L}^{-1}\right)$ & Species & $\begin{array}{l}\text { Duration } \\
\text { (h) }\end{array}$ & $\begin{array}{c}\text { Measured } \\
\mathrm{EC}_{50} \\
\left(\mathrm{mg} \mathrm{L}^{-1}\right) \\
\end{array}$ & $\begin{array}{c}\text { Nominal } \\
E C_{50} \\
\left(\mathrm{mg} \mathrm{L}^{-1}\right) \\
\end{array}$ & Classification \\
\hline tert-Nonyl mercaptan & $25360-10-5$ & & 4.59 & $9.60 \mathrm{E}+00$ & & & & & 4 \\
\hline Dibutyl phthalate & $84-74-2$ & 278.34 & 4.57 & $1.00 \mathrm{E}+01$ & $\begin{array}{l}\text { Scenedesmus subsppcatus } \\
\text { Scenedesmus subspicatus }\end{array}$ & 72 & & $\begin{array}{l}1.20 \\
9.00\end{array}$ & 1 \\
\hline Octene & 25377-83-7 & & 4.57 & $4.10 \mathrm{E}+00$ & & & & & 4 \\
\hline Nonene & $27215-95-8$ & & 4.55 & $3.62 \mathrm{E}+00$ & & & & & 4 \\
\hline $\begin{array}{l}\text { 2-Propenoic acid, 2-methyl-, 2-ethyl-2-[([2-methyl-1-oxo-2- } \\
\text { propenyl)oxylmethyl]-1,3-propanediyl ester }\end{array}$ & $3290-92-4$ & 338.40 & 4.50 & $1.30 \mathrm{E}+00$ & & & & & 4 \\
\hline \multirow[t]{3}{*}{ Phenol, 2,6-bis(1,1-dimethylethyl)- } & $128-39-2$ & 206.33 & 4.50 & $4.11 \mathrm{E}+00$ & & & & & 4 \\
\hline & & & & & Selenastrum capricornutum & 96 & 126.00 & & \\
\hline & & & & & Selenastrum capricornutum & 72 & 120.00 & & \\
\hline \multirow[t]{3}{*}{ Perfluorooctane sulfonic acid } & 1763-23-1 & 500.13 & 4.49 & $5.70 \mathrm{E}+02$ & $\begin{array}{l}\text { Selenastrum capricornutum } \\
\text { Anabaena flosaquae }\end{array}$ & $\begin{array}{l}96 \\
96\end{array}$ & 176.00 & 82.00 & 1 \\
\hline & & & & & Navicula pelliculosa & 96 & 305.00 & & \\
\hline & & & & & Skeletonema costatum & 96 & $=>3.2$ & & \\
\hline $\begin{array}{l}\text { Cyclotrisiloxane, hexamethyl- } \\
\text { Anthraquinone, 2-ethyl- }\end{array}$ & $541-05-9$ & 222.46 & 4.47 & $\begin{array}{l}1.60 \mathrm{E}+00 \\
4.05 \mathrm{E}-01\end{array}$ & Pseudokirchneriella subcapitata & 72 & & $>1.6$ & 3 \\
\hline Anthraquinone, 2-ethyl- & $84-51-5$ & & 4.37 & 4.05E-01 & & & & & 4 \\
\hline Dicyclohexylamine & $101-83-7$ & 181.32 & 4.37 & $8.00 \mathrm{E}+02$ & $\begin{array}{l}\text { Pseudokirchnerizella subcapitata } \\
\text { Desmodesmus subspicatus }\end{array}$ & $\begin{array}{l}72 \\
72\end{array}$ & 23.00 & $>1$ & 1 \\
\hline 4-tert-Butyl-2,6-dimethyl-3,5-dinitroacetophenone (Musk ketone) & $81-14-1$ & 294.30 & 4.30 & 4.60E-01 & Selenastrum capricornutum & 72 & 0.24 & & 1 \\
\hline 2-Butanone, peroxide & $1338-23-4$ & 210.22 & 4.30 & $2.70 \mathrm{E}+00-1.40 \mathrm{E}+04$ & Pseudokirchneriella subcapitata & 72 & 0.27 & 5.60 & 1 \\
\hline 1-Undecanol & $112-42-5$ & 172.31 & 4.28 & $8.00 \mathrm{E}+00$ & & & & & 4 \\
\hline 2,6-Dichlorotoluene & $118-69-4$ & 161.03 & 4.25 & $2.60 \mathrm{E}+01$ & Selenastrum capricornutum & 72 & 17.60 & & 1 \\
\hline 1-Octanethiol & $111-88-6$ & 146.29 & 4.21 & $1.98 \mathrm{E}-02$ & Pseudokirchneriella subcapitata & 72 & 0.04 & & 2 \\
\hline Dibutyl adipate & $105-99-7$ & 258.35 & 4.17 & $3.50 \mathrm{E}+01$ & Selenastrum capricornutum & 72 & 2.80 & & 1 \\
\hline Camphene & $79-92-5$ & 136.23 & 4.12 & $4.20 \mathrm{E}+00$ & Scenedesmus subspicatus & 72 & & $>=320$ & 3 \\
\hline m-Cresol, 6-tert-butyl- & $88-60-8$ & 164.24 & 4.11 & $4.20 \mathrm{E}+02$ & Selenastrum capricornutum & 72 & 1.84 & - & 1 \\
\hline $\begin{array}{l}\text { Propanoic acid, 2-methyl-, 2,2-dimethyl-1-(1-methylethyl)-1,3-propanediyl } \\
\text { ester }\end{array}$ & 6846-50-0 & 286.41 & 4.11 & $1.50 \mathrm{E}+01$ & Selenastrum capricornutum & 72 & & 8.00 & 1 \\
\hline Toluene, 2,4-dichloro- & 95-73-8 & 161.03 & 4.10 & $2.50 \mathrm{E}+01$ & Selenastrum capricornutum & 72 & 9.70 & & 1 \\
\hline Pentane, 2,2,4-trimethyl- & 540-84-1 & 114.23 & 4.09 & $2.44 \mathrm{E}+00$ & & & & & 4 \\
\hline Phenol, 2-(1,1-dimethylethyl)-4,6-dimethyl- & 1879-09-0 & 178.27 & 4.08 & $1.50 \mathrm{E}+02$ & Selenastrum capricornutum & 72 & 3.60 & & 1 \\
\hline Benzene, 1,4-diethyl- & $105-05-5$ & 134.22 & 4.06 & $1.70 \mathrm{E}+01$ & Selenastrum capricornutum & 72 & & 29.00 & 2 \\
\hline Benzene, 1,2,4-trichloro- & $120-82-1$ & 181.45 & 4.02 & $3.60 \mathrm{E}+01-4.80 \mathrm{E}+01$ & Selenastrum capricornutum & 96 & 1.40 & & 1 \\
\hline 1,1'-Biphenyl & $92-52-4$ & 154.21 & 4.01 & $7.48 \mathrm{E}+00$ & Pseudokirchneriella subcapitata & 72 & 0.78 & & 1 \\
\hline 3-Buten-2-one, 4-(2,6,6-trimethyl-1-cyclohexen-1-yl)- & 79-77-6 & 192.30 & 4.00 & $1.69 \mathrm{E}+02$ & Scenedesmus subspicatus & 72 & & 22.20 & 1 \\
\hline 3,5,9-Undecatrien-2-one, 6,10-dimethyl- & $141-10-6$ & 192.30 & 4.00 & $9.70 \mathrm{E}+01$ & Scenedesmus subspicatus & 72 & 2.02 & & 1 \\
\hline Heptene & 25339-56-4 & & 3.99 & $1.82 \mathrm{E}+01$ & & & & & 4 \\
\hline Isooctyl acrylate & $29590-42-9$ & 184.20 & 3.93 & $1.24 \mathrm{E}+01$ & & & & & 4 \\
\hline Acenaphthylene, 1,2-dihydro- & $83-32-9$ & & 3.92 & $3.80 \mathrm{E}-02$ & & & & & 4 \\
\hline Sulfone, bis(p-chlorophenyl) & $80-07-9$ & 287.16 & 3.90 & $8.60 \mathrm{E}-01$ & Selenastrum capricornutum & 72 & $>0.8$ & & 3 \\
\hline 2-Benzothiazolesulfeamide, N-(1,1-dimethylethyl)- & $95-31-8$ & 238.37 & 3.90 & $3.45 \mathrm{E}-01$ & & & & & 4 \\
\hline p-Phenylenediamine, N-(1-methylethyl)-N'-phenyl- & $101-72-4$ & 226.32 & 3.90 & $1.50 \mathrm{E}+01$ & Selenastrum capricornutum & 96 & & 0.50 & 1 \\
\hline 2-Ethylhexyl acrylate & 103-11-7 & 184.28 & 3.90 & $9.60 \mathrm{E}+00$ & Desmodesmus subspicatus & 72 & 1.71 & & 1 \\
\hline Hexane & $110-54-3$ & 86.18 & 3.90 & & & & & & 4 \\
\hline Linalylacetate & $115-95-7$ & 196.29 & 3.90 & $3.00 \mathrm{E}+01$ & Scenedesmus subspicatus & 72 & & 62.00 & 1 \\
\hline Phenol, 2,4,6-tribromo- & $118-79-6$ & 330.80 & 3.89 & $5.90 \mathrm{E}+01$ & Selenastrum capricornutum & 72 & & 1.60 & 1 \\
\hline Naphthalene, methyl- & $1321-94-4$ & 142.20 & 3.87 & & & & & & 4 \\
\hline Naphthalene, 2-methyl- & $91-57-6$ & 142.20 & 3.86 & $2.46 \mathrm{E}+01$ & Pseudokirchneriella subcapitata & 72 & 2.30 & & 1 \\
\hline 1H-Indene, 3a,4,7,7a-tetrahydro- & $3048-65-5$ & 120.19 & 3.83 & $3.74 \mathrm{E}+01$ & Pseudokirchneriella subcapitata & 72 & 7.00 & & 1 \\
\hline 2-Norbornene, 5-ethylidene- & $16219-75-3$ & 120.19 & 3.82 & $8.00 \mathrm{E}+01$ & Selenastrum capricornutum & 72 & 2.61 & & 1 \\
\hline Aniline, 4-nitro-N-phenyl- & $836-30-6$ & 214.00 & 3.82 & $4.10 \mathrm{E}+01$ & Selenastrum capricornutum & 96 & & 27.00 & 1 \\
\hline Silane, dichloromethylphenyl- & $149-74-6$ & & 3.80 & $7.85 \mathrm{E}+01$ & & & & & 4 \\
\hline Naphthalene, 1,2,3,4-tetrahydro- & $119-64-2$ & 132.21 & 3.78 & $4.50 \mathrm{E}+01$ & Desmodesmus subspicatus & 72 & & 11.00 & 1 \\
\hline 1-Pentanamine, N-pentyl- & 2050-92-2 & 157.30 & 3.76 & $4.44 \mathrm{E}+02$ & Selenastrum capricornutum & 72 & 1.70 & & 1 \\
\hline Anthraquinone, 1-amino- & $82-45-1$ & & 3.74 & $3.20 \mathrm{E}+01$ & Selenastrum capricornutum & 72 & & 0.25 & 1 \\
\hline 2-Ethylhexyl acetate & $103-09-3$ & 172.27 & 3.74 & $3.90 \mathrm{E}+00$ & Pseudokirchneriella subcapitata & 72 & $>21.9$ & & 3 \\
\hline Naphthalene & $91-20-3$ & 128.18 & 3.73 & $3.00 \mathrm{E}+01$ & Pseudokirchneriella subcapitata & 72 & 2.30 & & 1 \\
\hline Isodecanol & $25339-17-7$ & 158.29 & 3.71 & & & & & & 4 \\
\hline
\end{tabular}




\section{(Table S5. continued)}

\begin{tabular}{|c|c|c|c|c|c|c|c|c|c|}
\hline Chemicals name & CAS Reg. no & $\begin{array}{c}\text { Molecular } \\
\text { weight } \\
(\mathrm{g} \mathrm{mol}-1)\end{array}$ & $\log \mathrm{K}_{\mathrm{ow}}$ & Solubility (mg L $\left.{ }^{-1}\right)$ & Species & $\begin{array}{l}\text { Duration } \\
\text { (h) }\end{array}$ & $\begin{array}{c}\text { Measured } \\
\mathrm{EC}_{50} \\
\left(\mathrm{mg} \mathrm{L}^{-1}\right) \\
\end{array}$ & $\begin{array}{c}\text { Nominal } \\
\mathrm{EC}_{50} \\
\left(\mathrm{mg} \mathrm{L}^{-1}\right)\end{array}$ & Classification \\
\hline $\begin{array}{l}\text { 1-Heptanol, 2-propyl- } \\
\text { Diphenyl tolyl phosphate }\end{array}$ & $\begin{array}{l}10042-59-8 \\
26444-49-5\end{array}$ & 158.29 & $\begin{array}{l}3.71 \\
3.70\end{array}$ & $2.40 \mathrm{E}+00$ & Selenastrum capricornutum & 72 & 0.99 & & $\begin{array}{l}4 \\
1\end{array}$ \\
\hline Phenol, 4-(1,1,3,3-tetramethylbutyl)- & 140-66-9 & 206.33 & 3.70 & $1.90 \mathrm{E}+01$ & $\begin{array}{l}\text { Selenastrum capricornutum } \\
\text { Printz }\end{array}$ & 96 & & 1.90 & 1 \\
\hline $\begin{array}{l}\text { Silane, triethoxyoctyl- } \\
\text { Decanamine, N,N-dimethyl-, N-oxide } \\
\text { Amines, C10-16-alkyldimethyl, N-oxides }\end{array}$ & $\begin{array}{l}2943-75-1 \\
2605-79-0 \\
70592-80-2\end{array}$ & $\begin{array}{l}201.36 \\
201.36\end{array}$ & $\begin{array}{l}3.70 \\
3.69 \\
3.69\end{array}$ & $\begin{array}{l}1.30 \mathrm{E}-01 \\
3.04 \mathrm{E}+01 \\
3.04 \mathrm{E}+01\end{array}$ & Pseudokirchneriella subcapitata & 72 & & $>0.13$ & $\begin{array}{l}3 \\
4 \\
4\end{array}$ \\
\hline $\begin{array}{l}\text { Amines, (10-16-akkyldimethyl, } \mathrm{N} \text {-oxides } \\
\text { Isooctyl mercaptoacetate }\end{array}$ & 25103-09-7 & & $\begin{array}{l}3.68- \\
3.96\end{array}$ & $1.06 \mathrm{E}+01$ & & & & & 4 \\
\hline $\begin{array}{l}\text { N-(2-methylbutyl)-1-pentanamine } \\
4,4 \text {-Methylenebis-(2-chlorobenzenamine) }\end{array}$ & $\begin{array}{c}61361-18-0 \\
101-14-4\end{array}$ & 157.30 & $\begin{array}{l}3.68 \\
3.66\end{array}$ & $5.09 \mathrm{E}-01$ & $\begin{array}{l}\text { Pseudokirchneriella subcapitata } \\
\text { Chrorella vulgaris }\end{array}$ & $\begin{array}{c}72 \\
3\end{array}$ & $\begin{array}{l}>0.85 \\
21.27\end{array}$ & & $\begin{array}{l}4 \\
3\end{array}$ \\
\hline Cumene & $98-82-8$ & 120.19 & 3.66 & $7.50 \mathrm{E}+01$ & $\begin{array}{l}\text { Chlamydomonas angulosa } \\
\text { Selenastrum capricornutum }\end{array}$ & $\begin{array}{c}3 \\
72\end{array}$ & $\begin{array}{l}8.80 \\
2.60\end{array}$ & & 1 \\
\hline $\begin{array}{l}\text { Cyclopropanecarboxylic acid, 3-(2,2-dichloroethenyl)-2,2-dimethyl-, methyl } \\
\text { ester } \\
\text { Benzene, 1,2,4-trimethyl- }\end{array}$ & $61898-95-1$ & & $\begin{array}{l}3.66 \\
3.63\end{array}$ & $5.30 \mathrm{E}+01$ & Pseudokirchneriella subcapitata & 72 & 8.30 & & 1 \\
\hline Pyridine, 2,3,5,6-tetrachloro- & $2402-79-1$ & 216.87 & $\begin{array}{l}3.03 \\
3.63\end{array}$ & $2.94 \mathrm{E}+01-3.02 \mathrm{E}+01$ & $\begin{array}{l}\text { Selenastrum capricornutum } \\
\text { Selenastrum capricornutum }\end{array}$ & $\begin{array}{l}72 \\
96\end{array}$ & $\begin{array}{l}9.70 \\
8.50\end{array}$ & & $\begin{array}{l}4 \\
1\end{array}$ \\
\hline $\begin{array}{l}\text { 2-Methyl-N-(2-methylbutyl)-1-butanamine } \\
\text { Silane, trichlorophenyl-- } \\
\text { Phenol, 2-sec-butyl-4,6-dinitro- }\end{array}$ & $\begin{array}{c}27094-65-1 \\
98-13-5 \\
88-85-7\end{array}$ & $\begin{array}{l}157.30 \\
240.21\end{array}$ & $\begin{array}{l}3.61 \\
3.60 \\
3.57\end{array}$ & $\begin{array}{l}7.85 \mathrm{E}+02 \\
3.45 \mathrm{E}+01\end{array}$ & $\begin{array}{l}\text { Pseudokirchneriella subcapitata } \\
\text { Pseudokirchneriella subcapitata }\end{array}$ & $\begin{array}{l}72 \\
72\end{array}$ & 1.40 & $>100$ & $\begin{array}{l}4 \\
3 \\
1\end{array}$ \\
\hline 1-Octene & $111-66-0$ & 112.21 & $\begin{array}{l}3.50- \\
4.60\end{array}$ & 4.10E+00 & Selenastrum capricornutum & 96 & & 200.00 & 2 \\
\hline $\begin{array}{l}\text { 2,6-Naphthalenedicarboxylic acid, dimethyl ester } \\
\text { Formic acid, chloro-, 2-ethylhexyl ester }\end{array}$ & $\begin{array}{c}840-65-3 \\
24468-13-1\end{array}$ & 192.69 & $\begin{array}{l}3.50 \\
3.50\end{array}$ & $\begin{array}{l}1.50 \mathrm{E}-01 \\
4.90 \mathrm{E}+01\end{array}$ & Selenastrum capricornutum & 72 & & $>0.1$ & $\begin{array}{l}3 \\
4\end{array}$ \\
\hline Tributyl phosphate & $126-73-8$ & 266.31 & 3.50 & $4.00 \mathrm{E}+02$ & $\begin{array}{l}\text { Scenedesmus subspicatus } \\
\text { Selenastrum capricornutum }\end{array}$ & $\begin{array}{l}72 \\
96\end{array}$ & & $\begin{array}{l}2.80 \\
4.40\end{array}$ & 1 \\
\hline Phenol, 2-sec-butyl- & $89-72-5$ & 150.22 & 3.49 & $1.52 \mathrm{E}+03$ & $\begin{array}{l}\text { Pseudokirchneriella subcapitata } \\
\text { Pseudokirchnerella subcapitata }\end{array}$ & $\begin{array}{l}72 \\
72\end{array}$ & & $\begin{array}{l}6.90 \\
10.00\end{array}$ & 1 \\
\hline $\begin{array}{l}\text { Benzenesulfonyl chloride, 4-methyl- } \\
\text { Benzene, (1-methylethenyl)- }\end{array}$ & $\begin{array}{l}98-59-9 \\
98-83-9\end{array}$ & 190.65 & $\begin{array}{l}3.49 \\
3.48\end{array}$ & $\begin{array}{l}5.12 \mathrm{E}+01 \\
1.00 \mathrm{E}+02\end{array}$ & $\begin{array}{l}\text { Selenastrum capricornutum } \\
\text { Selenastrum capricornutum }\end{array}$ & $\begin{array}{l}72 \\
72\end{array}$ & & $\begin{array}{l}>100 \\
52.60\end{array}$ & $\begin{array}{l}3 \\
1\end{array}$ \\
\hline Propanoic acid, 2-methyl-, monoester with 2,2,4-trimethyl-1,3-pentanediol & $25265-77-4$ & 216.32 & 3.47 & $8.58 \mathrm{E}+02$ & $\begin{array}{l}\text { Selenastrum capricornutum } \\
\text { Scenedesmus subspicatus }\end{array}$ & $\begin{array}{l}72 \\
96\end{array}$ & 18.40 & 42.90 & 1 \\
\hline Benzene, 1,4-dichloro- & $106-46-7$ & 147.00 & 3.44 & $6.00 \mathrm{E}+01-7.00 \mathrm{E}+01$ & $\begin{array}{l}\text { Scenedesmus pannonicus } \\
\text { Selenastrum capricornutum } \\
\text { Scenedesmus subspicatus }\end{array}$ & $\begin{array}{l}72 \\
96 \\
48\end{array}$ & $\begin{array}{l}31.00 \\
1.60\end{array}$ & 38.00 & 1 \\
\hline Cyclohexane & $110-82-7$ & 84.16 & 3.44 & $5.80 \mathrm{E}+01$ & $\begin{array}{l}\text { Selenastrum capricornutum } \\
\text { Selenastrum capricornutum }\end{array}$ & $\begin{array}{l}72 \\
72\end{array}$ & $\begin{array}{l}>4.4 \\
3.40\end{array}$ & & 1 \\
\hline Benzoyl peroxide & $94-36-0$ & 242.24 & 3.43 & $9.10 \mathrm{E}+00$ & $\begin{array}{l}\text { Selenastrum capricornutum } \\
\text { Pseudokirchneriella subcapitata }\end{array}$ & $\begin{array}{l}72 \\
72\end{array}$ & $\begin{array}{l}0.44 \\
0.83\end{array}$ & & 1 \\
\hline $\begin{array}{l}\text { Methacrylic acid, 3-(trichlorosilyl)propyl ester } \\
\text { Toluene, 2-chloro- } \\
\text { Benzene, 1,3,5-trimethyl- } \\
\text { 1-Hexanol, 3,5,5-trimethyl- }\end{array}$ & $\begin{array}{l}7351-61-3 \\
95-49-8 \\
108-67-8 \\
3452-97-9\end{array}$ & $\begin{array}{l}126.58 \\
120.20\end{array}$ & $\begin{array}{l}3.43 \\
3.42 \\
3.42 \\
3.42\end{array}$ & $\begin{array}{c}9.87 \mathrm{E}+01 \\
4.70 \mathrm{E}+01 \\
4.00 \mathrm{E}+01-7.50 \mathrm{E}+01 \\
4.50 \mathrm{E}+02\end{array}$ & $\begin{array}{l}\text { Scenedesmus subspicatus } \\
\text { Selenastrum capricornutum }\end{array}$ & 72 & 33.30 & $>100$ & $\begin{array}{l}4 \\
3 \\
4 \\
1\end{array}$ \\
\hline 2-Hydroxy-3-napthoic acid & $92-70-6$ & 188.18 & $\begin{array}{l}3.40- \\
3.59\end{array}$ & $4.74 \mathrm{E}+02$ & Pseudokirchneriella subcapitata & 72 & 65.30 & & 1 \\
\hline Bisphenol A & $80-05-7$ & 228.29 & 3.40 & $3.00 \mathrm{E}+02$ & $\begin{array}{l}\text { Pseudokirchneriella Subcapitata } \\
\text { Pseudokichneriella Subcapitata } \\
\text { Skeletonema costatum } \\
\text { Selenastrum capricornutum }\end{array}$ & $\begin{array}{l}96 \\
96 \\
96 \\
96\end{array}$ & & $\begin{array}{l}2.50 \\
2.73 \\
1.10 \\
2.20\end{array}$ & 1 \\
\hline Benzene, 1,2-dichloro- & $95-50-1$ & 147.00 & 3.40 & $1.56 \mathrm{E}+02$ & $\begin{array}{l}\text { Selennastrum c capricornutum } \\
\text { Selenastrum capricornutum } \\
\text { Skeletonema costatum }\end{array}$ & $\begin{array}{l}96 \\
96 \\
96\end{array}$ & & $\begin{array}{l}91.60 \\
44.20\end{array}$ & 1 \\
\hline $\begin{array}{l}\text { DL-menthol } \\
\text { L-Menthol } \\
\text { (+)-Menthol }\end{array}$ & $\begin{array}{l}1490-04-6 \\
2216-51-5 \\
15356-60-2\end{array}$ & $\begin{array}{l}156.27 \\
156.27\end{array}$ & $\begin{array}{l}3.40 \\
3.40 \\
3.40\end{array}$ & $\begin{array}{c}5.08 \mathrm{E}+02 \\
4.31 \mathrm{E}+02 \\
4.20 \mathrm{E}+02-5.00 \mathrm{E}+02\end{array}$ & Scenedesmus subspicatus & 72 & 21.40 & & $\begin{array}{l}4 \\
1 \\
4\end{array}$ \\
\hline Ethene, tetrachloro- & $127-18-4$ & 165.83 & 3.40 & $1.60 \mathrm{E}+02$ & $\begin{array}{l}\text { Chlamydomonas reinhardii } \\
\text { Skeletonema costatum }\end{array}$ & $\begin{array}{l}72 \\
72\end{array}$ & & $\begin{array}{c}3.64 \\
500.00\end{array}$ & 1 \\
\hline $\begin{array}{l}\text { Hexene } \\
1 \text {-Hexene }\end{array}$ & $\begin{array}{c}25264-93-1 \\
592-41-6\end{array}$ & 84.16 & $\begin{array}{l}3.39 \\
3.39\end{array}$ & $\begin{array}{l}5.00 \mathrm{E}+01 \\
5.00 \mathrm{E}+01\end{array}$ & & & & & $\begin{array}{l}4 \\
4 \\
\end{array}$ \\
\hline
\end{tabular}


(Table S5. continued)

\begin{tabular}{|c|c|c|c|c|c|c|c|c|c|}
\hline Chemicals name & CAS Reg. no & $\begin{array}{c}\text { Molecular } \\
\text { weight } \\
\left(\mathrm{g} \mathrm{mol}^{-1}\right)\end{array}$ & $\log \mathrm{K}_{\mathrm{ow}}$ & Solubility $\left(\mathrm{mg} \mathrm{L}^{-1}\right)$ & Species & $\begin{array}{l}\text { Duration } \\
\text { (h) }\end{array}$ & $\begin{array}{c}\text { Measured } \\
\mathrm{EC}_{50} \\
\left(\mathrm{mg} \mathrm{L}^{-1}\right)\end{array}$ & $\begin{array}{c}\text { Nominal } \\
\mathrm{EC}_{50} \\
\left(\mathrm{mg} \mathrm{L}^{-1}\right)\end{array}$ & Classification \\
\hline Pentane & $109-66-0$ & & 3.39 & $3.85 \mathrm{E}+01$ & Selenastrum capricornutum & 72 & 7.51 & & 1 \\
\hline Dibutyl maleate & $105-76-0$ & & 3.38 & $1.73 \mathrm{E}+02$ & Scenedesmus subspicatus & 72 & & 6.20 & 1 \\
\hline Butane, 1,1'-oxybis- & $142-96-1$ & 130.23 & 3.35 & $1.13 \mathrm{E}+02$ & & & & & 4 \\
\hline Neodecanoyl chloride & $40292-82-8$ & 190.72 & 3.35 & $6.77 \mathrm{E}+01$ & & & & & 4 \\
\hline Toluene, 4-chloro- & $106-43-4$ & 126.59 & 3.33 & $4.00 \mathrm{E}+01$ & Desmodesmus subspicatus & 72 & $>0.96$ & & 3 \\
\hline Benzene, 1-chloro-2-(chloromethyl)- & $611-19-8$ & 161.03 & 3.32 & $1.00 \mathrm{E}+02$ & Selenastrum capricornutuma & 72 & 0.78 & & 1 \\
\hline Thymol & $89-83-8$ & & 3.30 & & 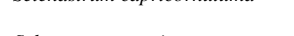 & & & & 4 \\
\hline Phenol, 4-tert-butyl- & $98-54-4$ & 150.22 & 3.29 & 6.10E+02 & Selenastrum capricornutum & 72 & 22.70 & & 1 \\
\hline 2,6-di-tert-Butyl-4-ethylphenol & $4130-42-1$ & & 3.27 & $2.10 \mathrm{E}+01$ & & & & & 4 \\
\hline Cyclohexanamine, 4,4'-methylenebis- & $1761-71-3$ & 210.37 & 3.26 & $6.42 \mathrm{E}+02$ & Desmodesmus subspicatus & 72 & & 141.42 & 1 \\
\hline Phenol, 2,4-dichloro- & $120-83-2$ & 163.00 & 3.25 & $4.50 \mathrm{E}+03$ & $\begin{array}{l}\text { Chlorella pyrenoidosa } \\
\text { Scenedesmus obliquus }\end{array}$ & 72 & & $\begin{array}{l}21.00 \\
33-39\end{array}$ & 1 \\
\hline Silane, (3-chloropropyl)trichloro- & $2550-06-3$ & & 3.24 & $6.44 \mathrm{E}+01$ & & & & & 4 \\
\hline Silane, tetramethyl- & $75-76-3$ & & 3.24 & $1.96 \mathrm{E}+01$ & Scenedesmus subspicatus & 72 & & $>0.0079$ & 3 \\
\hline Diallyl phthalate & $131-17-9$ & 246.26 & 3.23 & $1.48 \mathrm{E}+02$ & $\begin{array}{l}\text { Scenesdesmus subspicatus } \\
\text { Slpopastrum caricornutum }\end{array}$ & 72 & 1490 & & 1 \\
\hline Isononanol & $27458-94-2$ & 144.26 & 3.22 & & & & 14.90 & & 4 \\
\hline Diphenyl carbonate & $102-09-0$ & 214.22 & 3.21 & $1.30 \mathrm{E}+01$ & Desmodesmus subspicatus & 72 & 0.90 & & 1 \\
\hline Pentane, 2-methyl- & $107-83-5$ & 86.18 & 3.21 & $1.00 \mathrm{cos}$ & 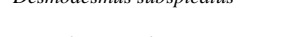 & 12 & 0.0 & & 4 \\
\hline Hexamethylene diisocyanate & $822-06-0$ & 168.20 & 3.20 & & Scenedesmus subspicatus & 72 & & $>77.4$ & 3 \\
\hline 1,3 -Xylene & $108-38-3$ & 106.17 & 3.20 & $1.61 \mathrm{E}+02$ & Selenastrum capricormutum & 72 & & 4.90 & 1 \\
\hline Peroxide, bis(tert-butyl)- & $110-05-4$ & & 3.20 & $1.71 \mathrm{E}+02$ & Pseudokirchneriella subcapitata & 72 & 36.00 & & 1 \\
\hline Benzene, 1,2-dichloro-3-nitro- & $3209-22-1$ & 192.00 & 3.20 & $7.41 \mathrm{E}+01$ & Selenastrum capricormutum & 72 & & 15.00 & 1 \\
\hline 1,4-Xylene & $106-42-3$ & 106.17 & 3.15 & $1.62 \mathrm{E}+02$ & Selenastrum capricornutum & 72 & & 3.20 & 1 \\
\hline Benzene, ethyl- & $100-41-4$ & 106.20 & 3.15 & $1.52 \mathrm{E}+02$ & $\begin{array}{l}\text { Selanastrum capricornatum } \\
\text { Skeletonema costatum }\end{array}$ & $\begin{array}{l}96 \\
96\end{array}$ & & $\begin{array}{l}3.60 \\
7.70\end{array}$ & 1 \\
\hline Ethanol, 2,2'-(dodecyloxidoimino)bis- & 2530-44-1 & 289.46 & 3.13 & $2.99 \mathrm{E}+01$ & Selenastrum capricornutum & 72 & & 0.18 & 1 \\
\hline Silane, (3-chloropropyl)triethoxy- & $5089-70-3$ & & 3.13 & $1.13 \mathrm{E}+02$ & Scenedesmus subspicatus & 72 & & $>819$ & 3 \\
\hline o-Xylene & $95-47-6$ & 106.17 & 3.12 & $1.78 \mathrm{E}+02$ & Selenastrum capricornutum & 72 & 4.70 & & 1 \\
\hline Stannane, dibutylbis[(1-oxododecyl)oxy]- & $77-58-7$ & & 3.12 & 7.00E-02 & Scenedesmus subspicatus & 72 & & $>3.3$ & 3 \\
\hline Propane, 1-(ethenyloxy)-2-methyl- & $109-53-5$ & & 3.10 & $7.20 \mathrm{E}+02$ & Desmodesmus subspicatus & 72 & 45.90 & & 1 \\
\hline Phenol, 4-chloro-2-methyl- & $1570-64-5$ & 142.59 & 3.09 & $2.30 \mathrm{E}+03$ & $\begin{array}{l}\text { Scenedesmus subspicatus } \\
\text { Scenedesmus subspicatus }\end{array}$ & 72 & & 15.00 & 1 \\
\hline Hexamethylene diacrylate & 13048-33-4 & 226.27 & 3.08 & & & & & & 4 \\
\hline \multirow[t]{3}{*}{ Dodecanedioic acid } & $693-23-2$ & 230.30 & 3.07 & $3.00 \mathrm{E}+01$ & & & & & 4 \\
\hline & & & & & Scenedesmus obliquus & 48 & 5.80 & & \\
\hline & & & & & Chlorella fusca & 24 & 0.32 & & \\
\hline \multirow{5}{*}{ 4-Nitrobenzene, 1,2-dichloro- } & & & & & Selenastrum obliquus & 96 & 10.50 & & \\
\hline & $99-54-1$ & 192.00 & 3.04 & $1.21 \mathrm{E}+02$ & Selenastrum capricornutum & 96 & 1.00 & & 1 \\
\hline & & & & & Scenedesmus obliquus & 48 & 5.80 & & \\
\hline & & & & & Haematococcus pluvialis & 4 & 2.00 & & \\
\hline & & & & & Selenastrum capricornutum & 72 & 31.20 & & \\
\hline \multirow[t]{2}{*}{ Butyl methacrylate } & $97-88-1$ & 142.20 & 3.03 & 3.60E+02 & Pseudokirchneriella subcapitata & 72 & & $>200$ & 1 \\
\hline & & & & & Pseudokirchneriella subcapitata & 5 & 934.00 & & \\
\hline 1,3-Butadiene, 2,3-dichloro- & $1653-19-6$ & 122.98 & 3.02 & & Desmodesmus subspicatus & 72 & $>2.1$ & & 3 \\
\hline Benzene, (trifluoromethyl)- & 98-08-8 & & 3.01 & $1.92 \mathrm{E}+02$ & Pseudokirchneriella subcapitata & 72 & 5.40 & & 1 \\
\hline Dibutyltin, maleate- & $78-04-6$ & 346.99 & 3.01 & & Scenedesmus subspicatus & 72 & 4.10 & & 1 \\
\hline 1-Octanol & $111-87-5$ & 130.23 & 3.00 & $5.51 \mathrm{E}+02$ & & & & & 4 \\
\hline Cyclopentane & $287-92-3$ & 70.14 & 3.00 & $1.56 \mathrm{E}+02$ & & & & & 4 \\
\hline Citral & $5392-40-5$ & 152.23 & 3.00 & $5.90 \mathrm{E}+02$ & $\begin{array}{l}\text { Scenedesmus subspicatus } \\
\text { Scenedesmus subspicatus }\end{array}$ & $\begin{array}{l}72 \\
72\end{array}$ & & $\begin{array}{l}16.00 \\
19.00\end{array}$ & 1 \\
\hline Cyclohexene & $110-83-8$ & 82.14 & 2.99 & $2.50 \mathrm{E}+02$ & & & & & 4 \\
\hline 1,6-Octadien-3-ol, 3,7-dimethyl- & $78-70-6$ & 154.24 & 2.97 & $8.54 \mathrm{E}+02-1.59 \mathrm{E}+03$ & Scenedesmus subspicatus & 96 & & 88.30 & 1 \\
\hline 2-methylpropyl 2-methyl-2-propenoate & $97-86-9$ & 142.20 & 2.95 & $4.70 \mathrm{E}+02$ & $\begin{array}{l}\text { Selenastrum capricornutum } \\
\text { Pseudokirchneriella subcapitata }\end{array}$ & $\begin{array}{l}72 \\
96\end{array}$ & 44.00 & $10-100$ & 1 \\
\hline Styrene & $100-42-5$ & 104.15 & 2.95 & $3.00 \mathrm{E}+02$ & 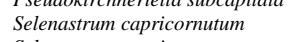 & $\begin{array}{l}90 \\
96\end{array}$ & & 6.30 & 1 \\
\hline Benzene, 1,4-dichloro-2-nitro- & $89-61-2$ & & 2.93 & $9.50 \mathrm{E}+01$ & Selenastrum capricornutum & 72 & 5.00 & & 1 \\
\hline Benzene, (trichloromethyl)- & $98-07-7$ & 195.48 & 2.92 & $1.00 \mathrm{E}+02$ & & & & & 4 \\
\hline Dodecane-12-lactam & $947-04-6$ & 197.32 & 2.92 & $3.00 \mathrm{E}+02$ & 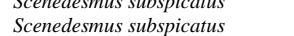 & 72 & 6.50 & & 1 \\
\hline
\end{tabular}




\section{(Table S5. continued)}

\begin{tabular}{|c|c|c|c|c|c|c|c|c|c|}
\hline Chemicals name & CAS Reg. no & $\begin{array}{c}\text { Molecular } \\
\text { weight } \\
\left(\mathrm{g} \mathrm{mol}^{-1}\right) \\
\end{array}$ & $\log \mathrm{K}_{\mathrm{ow}}$ & Solubility $\left(\mathrm{mg} \mathrm{L}^{-1}\right)$ & Species & $\begin{array}{l}\text { Duration } \\
\text { (h) }\end{array}$ & $\begin{array}{c}\text { Measured } \\
\mathrm{EC}_{50} \\
\left(\mathrm{mg} \mathrm{L}^{-1}\right) \\
\end{array}$ & $\begin{array}{c}\text { Nominal } \\
\mathrm{EC}_{50} \\
\left(\mathrm{mg} \mathrm{L}^{-1}\right) \\
\end{array}$ & Classification \\
\hline Guanidine, N,N'-bis(2-methylphenyl)- & $97-39-2$ & & 2.90 & $7.00 \mathrm{E}+01$ & Pseudokirchneriella subcapitata & 72 & 8.90 & & 1 \\
\hline Benzene, 2,4-dichloro-1-nitro- & $611-06-3$ & & 2.90 & $2.00 \mathrm{E}+02$ & Selenastrum capricornutum & 72 & & 2.00 & 1 \\
\hline 1-Octanamine & $111-86-4$ & 129.25 & 2.90 & $1.00 \mathrm{E}+06$ & Desmodesmus subspicatus & 72 & & 0.23 & 1 \\
\hline Phenol, 4-(1-methylethenyl)- & $4286-23-1$ & & 2.90 & $1.39 \mathrm{E}+03$ & Pseudokirchneriella subcapitata & 72 & 5.40 & & 1 \\
\hline $\begin{array}{l}\text { 2-Propenoic acid, 2-ethyl-2-[[(1-oxo-2-propenyl)oxy]methyl]-1,3- } \\
\text { propanediyl }\end{array}$ & $15625-89-5$ & 296.32 & 2.86 & & & & & & 4 \\
\hline (1) & $926-57-8$ & 125.00 & 2.84 & 3.63E+02 & $\begin{array}{l}\text { Pseudokirchneriella subcapitata } \\
\text { green algae } \\
\text { green algae }\end{array}$ & $\begin{array}{l}72 \\
96 \\
96\end{array}$ & $\begin{array}{c}650.00 \\
5.70 \\
104.60\end{array}$ & & 1 \\
\hline Methane, tetrachloro- & $56-23-5$ & 153.82 & 2.83 & $8.46 \mathrm{E}+02$ & Pseudokirchnerella subcapitata & 72 & 20.00 & & 1 \\
\hline Dibutylamine & $111-92-2$ & 129.25 & 2.83 & & $\begin{array}{l}\text { Desmodesmus subspicatus } \\
\text { Desmodesmus subspicatus }\end{array}$ & $\begin{array}{l}72 \\
72\end{array}$ & & $\begin{array}{l}16.91 \\
42.55\end{array}$ & 1 \\
\hline $\begin{array}{l}\text { Phosphoric acid, 2,2-bis(chloromethyl)-1,3-propanediyl tetrakis(2- } \\
\text { chloroethyl) }\end{array}$ & $38051-10-4$ & & 2.83 & $2.32 \mathrm{E}+02$ & Pseudokirchneriella subcapitata & 72 & & 35.00 & 1 \\
\hline 1-Hexanamine, 2-ethyl- & 104-75-6 & 129.24 & 2.82 & & & & & & 4 \\
\hline Butane, 1-chloro- & $109-69-3$ & & 2.82 & $3.70 \mathrm{E}+02$ & Selenastrum capricornutum & 72 & & $>1000$ & 3 \\
\hline Dicyclopentadiene & $77-73-6$ & 132.20 & 2.78 & & Selenastrum capricornutum & 72 & 27.00 & & 1 \\
\hline 1-Hexanol, 2-ethyl- & $104-76-7$ & & 2.73 & & & & & & 4 \\
\hline Butane, 2-methyl- & $78-78-4$ & & 2.72 & & green alga & 96 & 8.26 & & 1 \\
\hline Hexanal, 2-ethyl- & $123-05-7$ & 128.22 & 2.71 & $4.00 \mathrm{E}+02$ & $\begin{array}{l}\text { Scenedesmus subspicatus } \\
\text { Pheeodactslum tpicornutum }\end{array}$ & 96 & & 52.10 & 1 \\
\hline Aniline, 3,4-dichloro- & $95-76-1$ & 162.02 & 2.70 & $5.80 \mathrm{E}+02$ & $\begin{array}{l}\text { Pheoodactylum tricorrutum } \\
\text { Phaeodactylum tricornutum } \\
\text { Scenedesmus pannonicus }\end{array}$ & $\begin{array}{l}72 \\
96 \\
96\end{array}$ & $\begin{array}{l}1.10 \\
0.45 \\
4.80\end{array}$ & & 1 \\
\hline 3H-Indol-3-one, 2-(1,3-dihydro-3-oxo-2H-indol-2-ylidene)-1,2-dihydro- & $482-89-3$ & & 2.70 & $9.90 \mathrm{E}+02$ & Selenastrum capricornutum & 72 & & 6.50 & 1 \\
\hline 2-methylpropyl 2-methyl-2-propanoate & $97-85-8$ & 144.21 & 2.68 & $5.20 \mathrm{E}+02$ & Selenastrum capricornutum & 72 & & $>21.9$ & 3 \\
\hline 2-Butene, 2-methyl- & $513-35-9$ & 70.14 & 2.67 & $1.93 \mathrm{E}+02$ & Pseudokirchneriella subcapitata & 72 & & 12.00 & 1 \\
\hline Benzyl chloride & $100-44-7$ & & 2.66 & $1.20 \mathrm{E}+03$ & Selenastrum capricornutum & 72 & & 19.30 & 1 \\
\hline Toluene & $108-88-3$ & 92.14 & 2.65 & $5.15 \mathrm{E}+02$ & 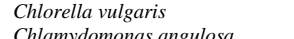 & 72 & & 207.00 & 1 \\
\hline Hexanoic acid, 2-ethyl- & $149-57-5$ & 144.22 & 2.64 & & Chlamydomonas angulosa & 72 & & 134.00 & 4 \\
\hline Diisobutylamine & $110-96-3$ & 129.25 & 2.63 & & Pseudokirchneriella subcapitata & 48 & & 16.00 & 1 \\
\hline Dehydrolinalool & 29171-20-8 & 152.24 & 2.61 & $2.45 \mathrm{E}+03$ & Scenedesmus subspicatus & 72 & & 46.00 & 1 \\
\hline Silane, dichloroethenylmethyl- & $124-70-9$ & & 2.60 & $8.02 \mathrm{E}+02$ & & & & & 4 \\
\hline Diisobutyl ketone & $108-83-8$ & & 2.56 & $4.30 \mathrm{E}+02$ & Selenastrum capricornutum & 96 & & 87.00 & 1 \\
\hline 2,2'-dimethyl-4,4'-methylenebis(cyclohexylamine) & $6864-37-5$ & & 2.51 & $3.60 \mathrm{E}+03$ & Scenedesmus subspicatus & 72 & & $>5$ & 3 \\
\hline Silane, dichloro(chloromethyl)methyl- & $1558-33-4$ & & 2.50 & $4.85 \mathrm{E}+02$ & & & & & 4 \\
\hline Silane, trichloroethyl- & $115-21-9$ & 163.51 & 2.50 & $9.87 \mathrm{E}+02$ & $\begin{array}{l}\text { Selenastrum capricornutum } \\
\text { Scenedesmus subspicatus }\end{array}$ & $\begin{array}{l}72 \\
72\end{array}$ & & $\begin{array}{l}>1053 \\
>=1000\end{array}$ & 3 \\
\hline Silane, chlorotrimethyl- & $75-77-4$ & & 2.48 & $1.01 \mathrm{E}+03$ & & & & & 4 \\
\hline Ethane, 1,1,1-trichloro- & $71-55-6$ & & 2.47 & $3.00 \mathrm{E}+02$ & Chlamydomonas reinhardtii & 72 & 0.54 & & 1 \\
\hline Benzoic acid, 4-methyl- & $99-94-5$ & & 2.44 & $3.49 \mathrm{E}+02$ & Pseudokirchneriella subcapitata & 72 & 74.00 & & 1 \\
\hline Ethene, trichloro- & $79-01-6$ & 131.39 & 2.42 & $1.10 \mathrm{E}+03$ & $\begin{array}{l}\text { Chlamydomonas reinhardii } \\
\text { Skeletonema costatum }\end{array}$ & $\begin{array}{l}72 \\
96\end{array}$ & & $\begin{array}{c}36.50 \\
150.00\end{array}$ & 1 \\
\hline $\begin{array}{l}\text { Isoprene } \\
1 \text {-Butene }\end{array}$ & $\begin{array}{c}78-79-5 \\
106-98-9\end{array}$ & 68.12 & $\begin{array}{l}2.42 \\
2.40\end{array}$ & $6.42 \mathrm{E}+02$ & $\begin{array}{l}\text { Pseudokirchneriella subcapitata } \\
\text { Petrom }\end{array}$ & 96 & 15.50 & & $\begin{array}{l}1 \\
4\end{array}$ \\
\hline 1-Butene, 2,3,4-trichloro- & $2431-50-7$ & 159.44 & 2.40 & $6.00 \mathrm{E}+02$ & & & & & 4 \\
\hline 2-Ethylhexyl mercaptoacetate & $7659-86-1$ & (5) & 2.40 & $4.73 \mathrm{E}+00$ & Pseudokirchneriella subcapitata & 72 & & 0.91 & 1 \\
\hline Hexanoyl chloride, 2-ethyl- & $760-67-8$ & 162.66 & 2.40 & $5.93 \mathrm{E}+02$ & Scenedesmus subspicatus & 96 & & 40.60 & 1 \\
\hline Hept-5-en-2-one, 6-methyl- & $110-93-0$ & 126.20 & 2.40 & $3.02 \mathrm{E}+03$ & Scenedesmus subspicatus & 72 & & 191.42 & 1 \\
\hline Silane, trichloroethenyl- & $75-94-5$ & & 2.40 & $1.34 \mathrm{E}+03$ & & & & & 4 \\
\hline Benzene, 1-chloro-4-nitro- & $100-00-5$ & 157.55 & 2.39 & 2.43E+02 & $\begin{array}{l}\text { Scenedesmus subspicatus } \\
\text { Chlorella pyrenoidosa }\end{array}$ & $\begin{array}{l}48 \\
96\end{array}$ & $\begin{array}{l}16.00 \\
4.90\end{array}$ & & 1 \\
\hline Ethane, 1,1,2,2-tetrachloro- & $79-34-5$ & 167.85 & 2.39 & $2.87 \mathrm{E}+03$ & $\begin{array}{l}\text { Scenedesmus subspicatus } \\
\text { Scenedesmus subspicatus }\end{array}$ & 72 & 47.00 & 76.00 & 1 \\
\hline Butyl acrylate & $141-32-2$ & 128.17 & 2.38 & $2.00 \mathrm{E}+03$ & $\begin{array}{l}\text { Scenedesmus subspicatus } \\
\text { Selenastrum capricornutum }\end{array}$ & 96 & & 5.20 & 1 \\
\hline Benzoic acid, 3-methyl- & $99-04-7$ & 136.15 & 2.37 & $1.00 \mathrm{E}+03$ & $\begin{array}{l}\text { Selenastrum capricornutum } \\
\text { Selenastrum capricornutum }\end{array}$ & $\begin{array}{l}72 \\
48\end{array}$ & & $\begin{array}{l}18.00 \\
15.00\end{array}$ & 1 \\
\hline Toluene, 4-nitro- & 99-99-0 & 137.14 & 2.37 & $3.45 \mathrm{E}+02$ & Chlorella pyrenoidosa & $\begin{array}{l}96 \\
48\end{array}$ & $\begin{array}{l}22.20 \\
2500\end{array}$ & & 1 \\
\hline 1-Butene, 3,4-dichloro- & $760-23-6$ & 125.00 & 2.37 & $1.10 \mathrm{E}+03$ & Selenastrum capricornutum & 72 & & 49.00 & 1 \\
\hline
\end{tabular}




\section{(Table S5. continued)}

\begin{tabular}{|c|c|c|c|c|c|c|c|c|c|}
\hline Chemicals name & CAS Reg. no & $\begin{array}{c}\text { Molecular } \\
\text { weight } \\
\left(\mathrm{g} \mathrm{mol}^{-1}\right)\end{array}$ & $\log \mathrm{K}_{\mathrm{ow}}$ & Solubility $\left(\mathrm{mg} \mathrm{L}^{-1}\right)$ & Species & $\begin{array}{l}\text { Duration } \\
\text { (h) }\end{array}$ & $\begin{array}{c}\text { Measured } \\
\mathrm{EC}_{50} \\
\left(\mathrm{mg} \mathrm{L}^{-1}\right)\end{array}$ & $\begin{array}{c}\text { Nominal } \\
\mathrm{EC}_{50} \\
\left(\mathrm{mg} \mathrm{L}^{-1}\right)\end{array}$ & Classification \\
\hline $\begin{array}{l}\text { Phenol, 4,4'-sulfonylbis- } \\
\end{array}$ & $80-09-1$ & & 2.36 & $7.70 \mathrm{E}+02$ & Pseudokirchneriella subcapitata & 72 & & 65.00 & 1 \\
\hline Phenol, 2,6-dimethyl- & $576-26-1$ & 122.17 & 2.36 & & & & & & 4 \\
\hline Propene, 2-methyl- & $115-11-7$ & 56.11 & 2.34 & 2.63E-01 & & & & & 4 \\
\hline 2-Butene, (2E)- & $624-64-6$ & 56.11 & 2.33 & $2.65 \mathrm{E}+02$ & algae & 96 & & 15.00 & 1 \\
\hline 2-Butene, (2Z)- & $590-18-1$ & 56.11 & 2.33 & $7.00 \mathrm{E}+02$ & & & & & 4 \\
\hline tert-Butyl acrylate & $1663-39-4$ & & 2.32 & $2.00 \mathrm{E}+03$ & Desmodesmus subspicatus & 72 & 14.60 & & 1 \\
\hline Toluene, 2-nitro- & $88-72-2$ & 137.14 & 2.30 & $4.37 \mathrm{E}+02$ & $\begin{array}{l}\text { Chlorella pyrenoidosa } \\
\text { Chlorella pvrenoidosa }\end{array}$ & $\begin{array}{l}72 \\
96\end{array}$ & $\begin{array}{l}22.00 \\
47.50\end{array}$ & & 1 \\
\hline Aniline, 4-(1-methylethyl)- & 99-88-7 & & 2.30 & $2.39 \mathrm{E}+03$ & Pseudokirchneriella subcapitata & 72 & 18.00 & & 1 \\
\hline Ethane, 1,1-dichloro-1-fluoro- & $1717-00-6$ & & 2.30 & $4.00 \mathrm{E}+03$ & Selenastrum capricornutum & 72 & $>44$ & & 3 \\
\hline 2-Propenamide & $79-06-1$ & 131.50 & 2.29 & $1.10 \mathrm{E}+03$ & Selenastrum capricornutum & 72 & & 67.70 & 1 \\
\hline 1-Butanethiol & $109-79-5$ & & 2.28 & $5.95 \mathrm{E}+02$ & & & & & 4 \\
\hline 2-Picoline, 5-ethyl- & $104-90-5$ & 121.18 & $2.27-$ & $1.20 \mathrm{E}+04$ & Selenastrum capricornutum & 72 & & 61.20 & 1 \\
\hline Butane, 1-isocyanato- & $111-36-4$ & 99.13 & 2.26 & $2.03 \mathrm{E}+05$ & & & & & 4 \\
\hline Dimethyl terephthalate & $120-61-6$ & 194.18 & 2.25 & $1.90 \mathrm{E}+01$ & $\begin{array}{l}\text { Scenedesmus subspicatus } \\
\text { Chlorella pvrenoidosa }\end{array}$ & $\begin{array}{l}72 \\
96\end{array}$ & 690 & $>32.3$ & 3 \\
\hline \multirow[t]{2}{*}{ Benzene, 1-chloro-2-nitro- } & $88-73-3$ & 157.55 & 2.24 & $4.41 \mathrm{E}+02$ & $\begin{array}{l}\text { Scenedesmus subspicatus } \\
\text { Scenedesmus obliquus. }\end{array}$ & $\begin{array}{l}48 \\
96\end{array}$ & $\begin{array}{l}34.00 \\
18.10\end{array}$ & & 1 \\
\hline & & & & & Scenedesmus pannonicus & 72 & 24.00 & & \\
\hline Silane, dichlorodimethyl- & $75-78-5$ & 129.06 & 2.24 & $1.75 \mathrm{E}+03$ & $\begin{array}{l}\text { Selenastrum capricornutum } \\
\text { Scenedesmus subspicatus }\end{array}$ & $\begin{array}{l}72 \\
72\end{array}$ & 555.00 & 806.00 & 1 \\
\hline Isobutyl acryliate & $106-63-8$ & & 2.22 & $2.00 \mathrm{E}+03$ & $\begin{array}{l}\text { Desmodesmus subspicatus } \\
\text { Desmestus }\end{array}$ & 72 & 3.18 & & 1 \\
\hline 1,2-ethanediyl 2-methyl-2-propenoate & $97-90-5$ & & 2.21 & & & & & & 4 \\
\hline o-Cresol & $95-48-7$ & 108.14 & 2.20 & $2.60 \mathrm{E}+04$ & & & & & 4 \\
\hline Chloroprene & $126-99-8$ & 100.14 & 2.20 & $2.56 \mathrm{E}+02-4.80 \mathrm{E}+02$ & & & & & 4 \\
\hline 3,4-Dimethylaniline & $95-64-7$ & & 2.17 & & Pseudokirchneriella subcapitata & 72 & 8.59 & & 1 \\
\hline Aniline, 2,6-dimethyl- & $87-62-7$ & & 2.17 & & Pseudokirchneriella subcapitata & 72 & $>100$ & & 3 \\
\hline 2,3-Dimethylaniline & $87-59-2$ & & 2.17 & & Pseudokirchneriella subcapitata & 72 & 41.40 & & 1 \\
\hline 3,5-Dimethylaniline & $108-69-0$ & 121.18 & 2.17 & 4.60E +03 & Pseudokirchneriella subcapitata & 72 & & 29.10 & 1 \\
\hline 2-Propenoic acid, 2-methyl, 2-propenyl ester & 0096-05-9 & & 2.15 & $2.20 \mathrm{E}+03$ & Pseudokirchneriella subcapitata & 96 & & 59.60 & 1 \\
\hline Stannane, trichlorooctyl- & $3091-25-6$ & 338.29 & 2.14 & $3.30 \mathrm{E}-01$ & Pseudokirchneriella subcapitata & 72 & 0.21 & & 1 \\
\hline 2-Propanethiol, 2-methyl- & $75-66-1$ & & 2.14 & $2.05 \mathrm{E}+03$ & Selenastrum capricornutum & 72 & & 24.00 & 1 \\
\hline Stannane, dibutyldichloro- & $683-18-1$ & 303.84 & 2.13 & $3.20 \mathrm{E}+02$ & Scenedesmus subspicatus & 72 & & 8.00 & 1 \\
\hline Benzene & $71-43-2$ & 78.11 & 2.13 & $1.80 \mathrm{E}+03$ & $\begin{array}{l}\text { Selenastrum capricornutum } \\
\text { Selenastrum capricornutum }\end{array}$ & $\begin{array}{l}72 \\
72\end{array}$ & & $\begin{array}{l}100.00 \\
29.00\end{array}$ & 1 \\
\hline 2-Butenedioic acid (2E)-, diethyl ester & $623-91-6$ & & 2.12 & $3.10 \mathrm{E}+03$ & Selenastrum capricornutum & 72 & & 1.10 & 1 \\
\hline 1-Propene, hexafluoro- & $116-15-4$ & 150.02 & 2.12 & & & & & & 4 \\
\hline Phenol, 3-methyl-4-nitro- & $2581-34-2$ & & 2.12 & $1.30 \mathrm{E}+01$ & Selenastrum capricornutum & 72 & & 8.60 & 1 \\
\hline 1-Propene, 3-chloro- & $107-05-1$ & & 2.10 & $3.60 \mathrm{E}+03$ & & & & & 4 \\
\hline 3-(Trimethoxysilyl)propyl methacrylate & $2530-85-0$ & & 2.10 & $8.26 \mathrm{E}+01$ & $\begin{array}{l}\text { Scenedesmus subspicatus } \\
\text { Scenedesmus subspicatus }\end{array}$ & $\begin{array}{l}72 \\
72\end{array}$ & $>536$ & $>100$ & 3 \\
\hline 3-(Trimethoxysilyl)propyl methacrylate & $1461-22-9$ & & 2.07 & $6.00 \mathrm{E}+00-1.00 \mathrm{E}+01$ & $\begin{array}{l}\text { Selenastrum capricornutum } \\
\text { Skeletonema costatum }\end{array}$ & $\begin{array}{l}96 \\
72\end{array}$ & & $\begin{array}{l}0.01 \\
0.00\end{array}$ & 1 \\
\hline Acrylonitrile & 107-13-1 & 53.06 & 2.05 & $7.35 \mathrm{E}+04$ & Scenedesmus subspicatus & 72 & & 3.10 & 1 \\
\hline Silane, triethoxy(2-methylpropyl)- & $17980-47-1$ & & 2.03 & $8.60 \mathrm{E}+01$ & & $\begin{array}{l}72 \\
72\end{array}$ & & $\begin{array}{l}14.10 \\
>36\end{array}$ & 3 \\
\hline 1-Hexanol & $111-27-3$ & 102.18 & 2.03 & $5.90 \mathrm{E}+03$ & $\begin{array}{l}\text { Scenedesmus subsplcatus } \\
\text { Psendokirchneriella subcapitata }\end{array}$ & 72 & 80.00 & & 1 \\
\hline Silanamine, trimethyl-N-trimethylsilyl- & $999-97-3$ & 161.40 & 2.02 & $7.61 \mathrm{E}+02$ & Scenedesmus subspicatus & 72 & 19.00 & & 1 \\
\hline Cyclohexylamine, N,N-dimethyl- & $98-94-2$ & & 2.01 & $1.34 \mathrm{E}+04$ & Desmodesmus subspicatus & 72 & $>2$ & & 3 \\
\hline Silane, trichloromethyl- & $75-79-6$ & & 2.01 & & $\begin{array}{l}\text { Selenastrum capricornutum } \\
\text { Psendekirchnoriello subcanitata }\end{array}$ & 72 & 555.00 & & 1 \\
\hline 2-Naphthol & $135-19-3$ & & $2.01-$ & $6.00 \mathrm{E}+02-9.00 \mathrm{E}+02$ & Pseudokirchneriella subcapitata & 72 & $>118$ & & 4 \\
\hline Propane, 1,2-dichloro- & & & $\begin{array}{l}2.84 \\
2.00\end{array}$ & $2.80 \mathrm{E}+03$ & Skeletonema costatum & 72 & & $14.7-16.3$ & $\begin{array}{l}4 \\
1\end{array}$ \\
\hline 1,3-Butadiene & $106-99-0$ & 54.09 & 1.99 & $7.35 \mathrm{E}+02$ & Skeletonema costatum & 72 & & & 4 \\
\hline Propane, 1,2,3-trichloro- & $96-18-4$ & & $\begin{array}{l}1.98- \\
2.54\end{array}$ & $1.75 \mathrm{E}+03$ & Selenastrum capricornutum & 72 & & $>101$ & 3 \\
\hline 2,4-Dinitrotoluene & $121-14-2$ & 182.14 & 1.98 & $3.91 E+03$ & & & & & $\begin{array}{l}4 \\
4\end{array}$ \\
\hline
\end{tabular}




\section{(Table S5. continued)}

\begin{tabular}{|c|c|c|c|c|c|c|c|c|c|}
\hline Chemicals name & CAS Reg. no & $\begin{array}{c}\text { Molecular } \\
\text { weight } \\
\left(\mathrm{g} \mathrm{mol}^{-1}\right)\end{array}$ & $\log \mathrm{K}_{\mathrm{ow}}$ & Solubility $\left(\mathrm{mg} \mathrm{L}^{-1}\right)$ & Species & $\begin{array}{l}\text { Duration } \\
\text { (h) }\end{array}$ & $\begin{array}{c}\text { Measured } \\
\mathrm{EC}_{50} \\
\left(\mathrm{mg} \mathrm{L}^{-1}\right)\end{array}$ & $\begin{array}{c}\text { Nominal } \\
\mathrm{EC}_{50} \\
\left(\mathrm{mg} \mathrm{L}^{-1}\right)\end{array}$ & Classification \\
\hline $\begin{array}{l}\text { Phenol, 3-methyl- } \\
\text { 12 4-Benzenetricarboxylic acid_cyclic-1, 2-anbydride }\end{array}$ & $\begin{array}{l}108-39-4 \\
552-30-7\end{array}$ & 108.14 & 1.96 & $\begin{array}{l}2.27 \mathrm{E}+04 \\
2.10 \mathrm{E}+04\end{array}$ & & & & & $\begin{array}{l}4 \\
4\end{array}$ \\
\hline $\begin{array}{l}\text { 1,2,4-Benzenetricarboxylic acid, cyclic-1,2-anhydride } \\
\text { Ethane, 2-chloro-1,1,1,2-tetrafluoro- }\end{array}$ & $\begin{array}{c}552-30-7 \\
2837-89-0\end{array}$ & 136.50 & $\begin{array}{l}1.95 \\
1.94\end{array}$ & $\begin{array}{l}2.10 \mathrm{E}+04 \\
1.45 \mathrm{E}+03\end{array}$ & Selenastrum capricornutum & 72 & & $>44$ & $\begin{array}{l}4 \\
3\end{array}$ \\
\hline Phenol, 4-methyl- & $106-44-5$ & 108.14 & 1.94 & $2.15 \mathrm{E}+04$ & $\begin{array}{l}\text { Scenedesmus subspicatus } \\
\text { Scenedesmus subspicatus }\end{array}$ & $\begin{array}{l}48 \\
48\end{array}$ & & $\begin{array}{l}4.60 \\
21.00\end{array}$ & 1 \\
\hline Methane, bromo- & 74-83-9 & & 1.94 & $1.61 \mathrm{E}+04$ & $\begin{array}{l}\text { Chlorella pyrenoidosa } \\
\text { Scenedesmus quadricauda }\end{array}$ & $\begin{array}{l}48 \\
48\end{array}$ & & $\begin{array}{l}5.00 \\
3.20\end{array}$ & 1 \\
\hline Silane, chlorodimethyl- & $1066-35-9$ & & 1.93 & & & & & & 4 \\
\hline Ethane, 1,1,2-trichloro- & $79-00-5$ & & 1.89 & $2.87 \mathrm{E}+03$ & Scenedesmus subspicatus & 72 & & 47.00 & 1 \\
\hline 2-Propenoic acid, 2-methyl-,1,2-ethanediylbis(oxy-2,1-ethanediyl)ester & $109-16-0$ & 286.33 & 1.88 & & & & & & 4 \\
\hline \multirow[t]{2}{*}{ Benzoic acid } & $65-85-0$ & & 1.88 & $2.90 \mathrm{E}+03$ & & & & & 4 \\
\hline & & & & & Selenastrum capricornutum & 72 & & $>110$ & \\
\hline \multirow[t]{2}{*}{ Ethyl methacrylate } & $97-63-2$ & & 1.87 & $4.69 \mathrm{E}+03$ & $\begin{array}{l}\text { Selenastrum capricornutum } \\
\text { Selenastrum capricornutum }\end{array}$ & $\begin{array}{l}72 \\
96\end{array}$ & 0.64 & $>72$ & 1 \\
\hline & & & & & Selenastrum capricornutum & 72 & & $>100$ & \\
\hline Aniline, 2-nitro- & $88-74-4$ & & 1.85 & $1.17 \mathrm{E}+03$ & $\begin{array}{l}\text { Chlorella vulgaris } \\
\text { Scenedesmus obliguus }\end{array}$ & $\begin{array}{c}6 \\
96\end{array}$ & $\begin{array}{l}91.26 \\
64.60\end{array}$ & & 1 \\
\hline 2-Butene & $107-01-7$ & 56.10 & 1.85 & & & & & & 4 \\
\hline Methacrylate, methyl- & $80-62-6$ & & 1.83 & $1.60 \mathrm{E}+04$ & Selenastrum capricornutum & 96 & 170.00 & & 1 \\
\hline 2,5-Dimethylaniline & $95-78-3$ & & 1.83 & & Pseudokirchneriella subcapitata & 72 & 30.00 & & 1 \\
\hline p-Phenylenediamine, $\mathrm{N}$-phenyl- & $101-54-2$ & & 1.82 & $5.04 \mathrm{E}+02$ & Desmodesmus subspicatus & 72 & & 4.80 & 1 \\
\hline Tripropylene glycol diacrylate & $42978-66-5$ & 300.35 & 1.82 & & & & & & 4 \\
\hline Butyl acetate & $123-86-4$ & & 1.82 & $1.40 \mathrm{E}+04$ & Microcystis aeruginosa & 72 & & 675.00 & 1 \\
\hline 1-Propanethiol & 107-03-9 & & 1.81 & $1.90 \mathrm{E}+03$ & & & & & 4 \\
\hline Thiophene & $110-02-1$ & & 1.81 & $3.02 \mathrm{E}+03$ & Pseudokirchneriella subcapitata & 72 & 113.00 & & 1 \\
\hline 2-Methylpropyl acetate & $110-19-0$ & & 1.78 & $6.00 \mathrm{E}+03$ & & & & & 4 \\
\hline 1-Propene & $115-07-1$ & & 1.77 & $2.00 \mathrm{E}+02$ & & & & & 4 \\
\hline Isophthalic acid & $121-91-5$ & & 1.76 & $1.30 \mathrm{E}+02$ & Scenedesmus subspicatus & 72 & & - & 3 \\
\hline Benzaldehyde, 4-methoxy- & $123-11-5$ & & 1.76 & $4.40 \mathrm{E}+03$ & Pseudokirchneriella subcapitata & 72 & 61.00 & 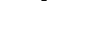 & 1 \\
\hline Ethane, 1,1,1-trifluoro- & $420-46-2$ & & 1.74 & $7.61 \mathrm{E}+02$ & Pseudokirchneriella subcapitata & 72 & 0.00 & $>44$ & 3 \\
\hline Disulfide, bis(dimethylthiocarbamoyl)- & $137-26-8$ & & 1.73 & $1.80 \mathrm{E}+01$ & $\begin{array}{l}\text { Pseudokirchnerella subcapitata } \\
\text { Psibla }\end{array}$ & 72 & & 0.19 & 1 \\
\hline Cyanuric chloride & $108-77-0$ & 184.41 & 1.70 & $4.40 \mathrm{E}+02$ & & & & & 4 \\
\hline Silane, dichloromethyl- & $75-54-7$ & & 1.70 & & & & & & 4 \\
\hline Guanidine, 1,3-diphenyl- & $102-06-7$ & & 1.69 & $4.75 \mathrm{E}+02$ & $\begin{array}{l}\text { Scenedesmus subspicatus } \\
\text { Selenastrum capricornutum }\end{array}$ & $\begin{array}{l}72 \\
96\end{array}$ & & $\begin{array}{l}7.50 \\
170\end{array}$ & 1 \\
\hline Methane, dibromo- & $74-95-3$ & & 1.68 & $9.00 \mathrm{E}+03$ & Pseudokirchneriella subcapitata & 72 & 150.00 & & 1 \\
\hline Butanoic acid, 2-ethyl & $88-09-5$ & & 1.68 & $1.70 \mathrm{E}+04$ & Pseudokirchneriella subcapitata & 72 & $>63$ & & 3 \\
\hline 2,4-Xylidine & $95-68-1$ & & 1.68 & & & & & & 4 \\
\hline 2-Pentanol, 4-methyl- & $108-11-2$ & 102.17 & 1.68 & $1.64 \mathrm{E}+04$ & Pseudokirchneriella subcapitata & 72 & 264.00 & & 1 \\
\hline 2-Cyclohexen-1-one, 3,5,5-trimethyl- & $78-59-1$ & & 1.67 & $1.45 \mathrm{E}+04$ & Scenedesmus subspicatus & 72 & & 475.00 & 1 \\
\hline Dipropylamine & $142-84-7$ & 101.19 & 1.67 & & & & & & 4 \\
\hline Phenol, 2,4-dinitro- & $51-28-5$ & & 1.67 & & & & & & 4 \\
\hline Benzaldehyde, 2-hydroxy- & $90-02-8$ & & 1.66 & $4.90 \mathrm{E}+03$ & Pseudokirchneriella subcapitata & 72 & 4.80 & & 1 \\
\hline Ethene, chlorotrifluoro- & $79-38-9$ & & 1.65 & $8.04 \mathrm{E}+02$ & & & & & 4 \\
\hline Ethane, 1-chloro-1,1-difluoro- & $75-68-3$ & 100.50 & $\begin{array}{l}1.64- \\
2.05\end{array}$ & $1.90 \mathrm{E}+03$ & & & & & 4 \\
\hline Hydroperoxide, 1-methyl-1-phenylethyl- & $80-15-9$ & & 1.60 & $1.43 \mathrm{E}+05$ & Pseudokirchneriella subcapitata & 72 & 3.10 & & 1 \\
\hline 1,3-Isobenzofurandione & $85-44-9$ & & 1.60 & $3.33 \mathrm{E}+03$ & Desmodesmus subspicatus & 72 & & $>=100$ & 3 \\
\hline Ethene, ethoxy- & $109-92-2$ & & 1.60 & $7.80 \mathrm{E}+03$ & Desmodesmus subspicatus & 72 & & $>1000$ & 3 \\
\hline Aniline, 4,4'-methylenebis- & $101-77-9$ & 198.30 & 1.59 & $1.25 \mathrm{E}+03$ & Scenedesmus subspicatus & 72 & & 11.00 & 1 \\
\hline Ethene, chloro- & $75-01-4$ & & 1.58 & $1.10 \mathrm{E}+03$ & & & & & 4 \\
\hline 2-Butoxyethyl acetate & $112-07-2$ & & 1.57 & $1.50 \mathrm{E}+04$ & $\begin{array}{l}\text { Scenedesmus subspicatus } \\
\text { Pseudokirchneriella subcapitata }\end{array}$ & 72 & & $>500$ & 1 \\
\hline tert-Amyl methyl ether & 994-05-8 & & 1.55 & $1.10 \mathrm{E}+04$ & $\begin{array}{l}\text { Pseudokirchneriella subcapitata } \\
\text { Pseudokirchneriella subcapitata }\end{array}$ & $\begin{array}{l}72 \\
72\end{array}$ & $\begin{array}{l}1570.00 \\
870.00\end{array}$ & & 1 \\
\hline Ethanol, 2-(hexyloxy)- & $112-25-4$ & & 1.55 & $9.90 \mathrm{E}+03$ & Scenedesmus subspicatus & 72 & & 198.00 & 1 \\
\hline Aniline, 3-nitro- & $99-09-2$ & 138.14 & 1.54 & $1.14 \mathrm{E}+03$ & Selenastrum capricornutum & 72 & & 20.00 & 1 \\
\hline Pyridine, 2-vinyl- & $100-69-6$ & & 1.54 & $2.67 \mathrm{E}+04$ & Pseudokirchneriella subcapitata & 72 & 62.00 & & 1 \\
\hline Carbonochloridic acid, 1-methylpropyl ester & $17462-58-7$ & 136.58 & 1.54 & $4.22 \mathrm{E}+03$ & & & & & 4 \\
\hline 1-Propanol, butoxy- & $29387-86-8$ & & 1.52 & $5.50 \mathrm{E}+04$ & & & & & 4 \\
\hline 2-Propanol, 1-phenoxy- & $770-35-4$ & 152.19 & 1.52 & $1.00 \mathrm{E}+04$ & Scenedesmus subspicatus & 72 & & $>100$ & 3 \\
\hline
\end{tabular}




\section{(Table S5. continued)}

\begin{tabular}{|c|c|c|c|c|c|c|c|c|c|}
\hline Chemicals name & CAS Reg. no & $\begin{array}{c}\text { Molecular } \\
\text { weight } \\
\left(\mathrm{g} \mathrm{mol}^{-1}\right) \\
\end{array}$ & $\log \mathrm{K}_{\mathrm{ow}}$ & Solubility $\left(\mathrm{mg} \mathrm{L}^{-1}\right)$ & Species & $\begin{array}{l}\text { Duration } \\
\text { (h) }\end{array}$ & $\begin{array}{c}\text { Measured } \\
\mathrm{ES}_{50} \\
\left(\mathrm{mg} \mathrm{L}^{-1}\right)\end{array}$ & $\begin{array}{l}\text { Nominal } \\
E_{50} \\
\left(\mathrm{mg} \mathrm{L}^{-1}\right)\end{array}$ & Classification \\
\hline Propanenitrile, 3-(trichlorosily)- & $1071-22-3$ & \multirow{3}{*}{68.00} & 1.52 & $1.37 \mathrm{E}+03$ & & & & & 4 \\
\hline 1,3-Pentadiene & $504-60-9$ & & 1.50 & $6.90 \mathrm{E}+02$ & Selenastrum capricornutum & 72 & & 293.90 & 1 \\
\hline Propylene glycol phenyl ether (beta isomer - primary alcohol) & 4169-04-4 & & 1.50 & $1.00 \mathrm{E}+04$ & Scenedesmus subspicatus & 72 & & $>100$ & 3 \\
\hline 1,4-Cyclohexanedimethanol & $105-08-8$ & \multirow{3}{*}{106.12} & 1.49 & $9.20 \mathrm{E}+05$ & Pseudokirchneriella subcapitata & 72 & & $>120$ & 3 \\
\hline Benzaldehyde & $100-52-7$ & & 1.48 & $6.55 \mathrm{E}+03$ & Scenedesmus & 24 & & 340.00 & 1 \\
\hline Ethane, pentafluoro- & 354-33-6 & & 1.48 & $4.32 \mathrm{E}-01-1.07 \mathrm{E}+00$ & & & & & 4 \\
\hline Phenol & $108-95-2$ & & 1.47 & $8.40 \mathrm{E}+04$ & $\begin{array}{l}\text { Selenastrum capricornutum } \\
\text { Selenastrum capricornutum }\end{array}$ & $\begin{array}{l}72 \\
96\end{array}$ & & $\begin{array}{c}61.10 \\
150.00\end{array}$ & 1 \\
\hline Silane, trichloro- & $10025-78-2$ & \multirow{4}{*}{101.19} & 1.46 & & & & & & 4 \\
\hline Triethylamine & $121-44-8$ & & 1.45 & & & & & & 4 \\
\hline Ethane, 1,2-dichloro- & $107-06-2$ & & 1.45 & $8.49 \mathrm{E}+03-9.00 \mathrm{E}+03$ & Scenedesmus subspicatus & 72 & 189.00 & & 1 \\
\hline Ethane, chloro- & $75-00-3$ & & 1.43 & $5.74 \mathrm{E}+03$ & Scenedesmus subspicatus & 72 & & 39.00 & 1 \\
\hline \multirow{2}{*}{ Aniline, 2-methyl- } & & & & & $\begin{array}{l}\text { Selenastrum capricornutum } \\
\text { Chlorella avrenoidosa }\end{array}$ & 72 & 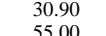 & & \\
\hline & $95-53-4$ & & 1.40 & $1.50 \mathrm{E}+04$ & $\begin{array}{l}\text { Chlorella pyrenoidosa } \\
\text { Scenedesmus obliquus }\end{array}$ & $\begin{array}{l}96 \\
48\end{array}$ & $\begin{array}{r}55.00 \\
490.00\end{array}$ & & 1 \\
\hline 2-Propanamine, N-(1-methylethyl)- & $108-18-9$ & 101.19 & 1.40 & & Selenastrum sp. & 96 & & 20.00 & 1 \\
\hline m-Toluidine & $108-44-1$ & \multirow[t]{3}{*}{107.16} & 1.40 & $1.00 \mathrm{E}+04$ & Selenastrum capricornutum & 72 & & 17.70 & 1 \\
\hline Hydroperoxide, 1,1-dimethylpropyl- & $3425-61-4$ & & 1.40 & & & & & & 4 \\
\hline Butyric anhydride & $106-31-0$ & & 1.39 & $4.56 \mathrm{E}+03$ & Scenedesmus subspicatus & 96 & & 42.90 & 1 \\
\hline Aniline, 4-methyl- & $106-49-0$ & \multirow{3}{*}{102.13} & 1.39 & $7.40 \mathrm{E}+03$ & $\begin{array}{l}\text { Scenedesmus obliquus } \\
\text { Scenedesmus quadricauda }\end{array}$ & $\begin{array}{l}48 \\
96\end{array}$ & & $\begin{array}{l}62.90 \\
8.00\end{array}$ & 1 \\
\hline Pentanoic acid & $109-52-4$ & & 1.39 & $2.40 \mathrm{E}+04$ & Pseudokirchneriella subcapitata & 72 & & 10.70 & 1 \\
\hline 1,3,5-Triazine-2,4-diamine, 6-phenyl- & $91-76-9$ & & 1.38 & $3.20 \mathrm{E}+02$ & $\begin{array}{l}\text { Selenastrum capricornutum } \\
\text { Scenedesmus subspicatus }\end{array}$ & $\begin{array}{l}72 \\
72\end{array}$ & & $\begin{array}{l}53.70 \\
22.00\end{array}$ & 1 \\
\hline Pentanal & $110-62-3$ & & 1.38 & $1.17 \mathrm{E}+04$ & $\begin{array}{l}\text { Pseudokirchnerilla subcapitata } \\
\text { Pseudokirchnerilla subcapitata }\end{array}$ & $\begin{array}{l}72 \\
72\end{array}$ & & $\begin{array}{l}32.40 \\
42.20\end{array}$ & 1 \\
\hline Benzoic acid, 4-hydroxy- & $99-96-7$ & & 1.37 & $6.00 \mathrm{E}+03$ & Selenastrum capricornutum & \multirow{4}{*}{$\begin{array}{l}72 \\
96\end{array}$} & & 68.50 & 1 \\
\hline 3-Penten-2-one, 4-methyl- (Mesityl oxide) & $141-79-7$ & 98.15 & 1.37 & $3.00 \mathrm{E}+04$ & \multirow[t]{3}{*}{ Pseudokirchnerella subcapitata } & & & \multirow[t]{3}{*}{120.00} & 1 \\
\hline Vanillin & $121-33-5$ & \multirow[t]{3}{*}{152.14} & 1.35 & $1.00 \mathrm{E}+04$ & & & & & 4 \\
\hline Trichloroacetic acid & $76-03-9$ & & 1.33 & \multirow{3}{*}{$2.00 \mathrm{E}+04$} & & & & & 4 \\
\hline Butanal, 3-methyl- & $590-86-3$ & & 1.32 & & Scenedesmus subspicatus & 72 & & 80.00 & 1 \\
\hline Ethanol, 2-(2-butoxyethoxy)-, acetate & $124-17-4$ & 204.27 & 1.30 & & $\begin{array}{l}\text { Scenedesmus subspicatus } \\
\text { Pseudokirchneriella subcapitata }\end{array}$ & 72 & & $\begin{array}{l}>500 \\
5000\end{array}$ & 1 \\
\hline 2H-Pyran, 3,4-dihydro-2-methoxy- & 4454-05-1 & 114.14 & 1.30 & $1.60 \mathrm{E}+04$ & $\begin{array}{l}\text { Sseudokirchneriella subcapitata } \\
\text { Scenedesmus subspicatus }\end{array}$ & $\begin{array}{l}72 \\
72\end{array}$ & & $\begin{array}{l}520.00 \\
>500\end{array}$ & 3 \\
\hline Aniline, 4-ethoxy- & $156-43-4$ & 137.18 & 1.28 & $2.10 \mathrm{E}+04$ & Selenastrum capricornutum & 72 & & 5.10 & 1 \\
\hline Ethanethiol & $75-08-1$ & \multirow{3}{*}{190.29} & 1.27 & $1.56 \mathrm{E}+04$ & \multirow{2}{*}{ Pseudokirchneriella subcapitata } & 72 & 3.00 & & 1 \\
\hline Ethanol, 2-[2-(hexyloxy)ethoxy]- & $112-59-4$ & & 1.27 & $1.70 \mathrm{E}+04$ & & & & & 4 \\
\hline Methane, dichloro- & $75-09-2$ & & 1.25 & $1.32 \mathrm{E}+04$ & Chlamydomonas sp. & 3 & 1478-2292 & & 1 \\
\hline Propanoic acid, 2-methyl-, anhydride & $97-72-3$ & & 1.24 & $1.60 \mathrm{E}+04$ & $\begin{array}{l}\text { Selenastrum capricornutum } \\
\text { Scenedesmus subspicatus }\end{array}$ & $\begin{array}{l}72 \\
96\end{array}$ & $\begin{array}{l}10.70 \\
42.90\end{array}$ & & 1 \\
\hline Propyl acetate & $109-60-4$ & & 1.24 & $2.00 \mathrm{E}+04$ & $\begin{array}{l}\text { Pseudokirchneriella subcapitata } \\
\text { Psiras }\end{array}$ & 72 & & $>1,000$ & 3 \\
\hline Ethene, 1,1-difluoro- & $75-38-7$ & 64.00 & 1.24 & $2.54 \mathrm{E}+02$ & & & & & 4 \\
\hline Propyl chloroformate & $109-61-5$ & 122.55 & 1.22 & $1.09 \mathrm{E}+04$ & & & & & 4 \\
\hline Ethyl acrylate & $140-88-5$ & & 1.18 & $1.50 \mathrm{E}+04$ & Scenedesmus subspicatus & 72 & & 48.00 & 1 \\
\hline Aniline, 2-methoxy- & $90-04-0$ & & 1.18 & & 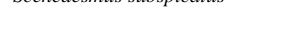 & 12 & & 70.00 & 4 \\
\hline Butanoic acid, 2-methyl- & $116-53-0$ & 102.13 & 1.18 & & & & & & 4 \\
\hline Terephthalic acid & $100-21-0$ & 102.15 & 1.16 & $1.90 \mathrm{E}+04$ & Scenedesmus subspicatus & 96 & $>927$ & & 3 \\
\hline Ethanol, 2-phenoxy- & $122-99-6$ & 138.17 & 1.16 & $2.89 \mathrm{E}+04$ & Scenedesmus subspicatus & 72 & $>500$ & & 3 \\
\hline Acetamide, N-phenyl- & $103-84-4$ & & 1.16 & $4.00 \mathrm{E}+03$ & Selenastrum capricornutum & 72 & 13.50 & & 1 \\
\hline Methyl isobutyl ketone & $108-10-1$ & 100.16 & 1.16 & $1.70 \mathrm{E}+04-2.04 \mathrm{E}+04$ & Scenedesmus subspicatus & 48 & & 2000.00 & 1 \\
\hline Phthalimide & $85-41-6$ & & 1.15 & $3.70 \mathrm{E}+02$ & Selenastrum capricornutum & 72 & 161.00 & & 1 \\
\hline 2-Propanol, 1-butoxy- & 5131-66-8 & & 1.15 & $5.50 \mathrm{E}+04$ & & & & & 4 \\
\hline 2,5,7,10-Tetraoxa-6-silaundecane, 6-ethenyl-6-(2-methoxyethoxy)- & $1067-53-4$ & & 1.14 & $5.00 \mathrm{E}+01-1.00 \mathrm{E}+06$ & & & & & 4 \\
\hline 2-(Dimethylamino)ethyl methacrylate & $2867-47-2$ & & 1.13 & $1.06 \mathrm{E}+05$ & Selenastrum capricornutum & 72 & 41.60 & & 1 \\
\hline 2-Propanol, 1-(2-butoxy-1-methylethoxy)- & 29911-28-2 & 190.29 & 1.13 & $4.50 \mathrm{E}+04$ & & & & & 4 \\
\hline Ethene & 74-85-1 & & 1.13 & $1.31 \mathrm{E}+02$ & & & & & 4 \\
\hline $\begin{array}{l}\text { Phosphoric acid, triethyl ester } \\
\text { Benpenemethanol }\end{array}$ & $\begin{array}{c}78-40-0 \\
10-5-6\end{array}$ & & 1.11 & $\begin{array}{l}\text { miscible } \\
400 \mathrm{E} \\
\mathrm{n}\end{array}$ & Scenedesmus subspicatus & 72 & & 900.00 & 1 \\
\hline $\begin{array}{l}\text { Benzenemethanol } \\
\text { Propanenitrile, 2.'-azobis 2-methyl- }\end{array}$ & $\begin{array}{l}100-51-6 \\
78-67-1\end{array}$ & & 1.10 & $4.00 \mathrm{E}+04$ & & 72 & & $>9.4$ & 4 \\
\hline Propanenitrule, 2,2-azobis[2-methyl- & $78-67-1$ & & 1.10 & $3.50 \mathrm{E}+02$ & Pseudokirchneriella subcapitata & 72 & & 6.10 & 1 \\
\hline
\end{tabular}


(Table S5. continued)

\begin{tabular}{|c|c|c|c|c|c|c|c|c|c|}
\hline Chemicals name & CAS Reg. no & $\begin{array}{c}\text { Molecular } \\
\text { weight } \\
\left(\mathrm{g} \mathrm{mol}^{-1}\right)\end{array}$ & $\log \mathrm{K}_{\mathrm{ow}}$ & Solubility $\left(\mathrm{mg} \mathrm{L}^{-1}\right)$ & Species & $\begin{array}{l}\text { Duration } \\
\text { (h) }\end{array}$ & $\begin{array}{c}\text { Measured } \\
\mathrm{EC}_{50} \\
\left(\mathrm{mg} \mathrm{L}^{-1}\right)\end{array}$ & $\begin{array}{c}\text { Nominal } \\
\mathrm{EC}_{50} \\
\left(\mathrm{mg} \mathrm{L}^{-1}\right) \\
\end{array}$ & Classification \\
\hline 1-Methylethyl carbonochloridiate & $108-23-6$ & 122.55 & 1.04 & $1.26 \mathrm{E}+04$ & & & \multirow{6}{*}{22.00} & \multirow{4}{*}{10.00} & 4 \\
\hline 1-methylethyl acetate & $108-21-4$ & & 1.02 & $3.09 \mathrm{E}+04$ & & & & & 4 \\
\hline Aniline, 3-methoxy- & $536-90-3$ & 123.00 & 1.01 & $2.05 \mathrm{E}+04$ & Selenestrum capricornutum & 72 & & & 1 \\
\hline Pyrocatechol & $120-80-9$ & & 1.01 & $4.49 \mathrm{E}+05$ & algae & 96 & & & 1 \\
\hline Cyclohexylamine, 3-aminomethyl-3,5,5-trimethyl- & 2855-13-2 & 170.30 & 0.99 & miscible & Scenedesmus subspicatus & 72 & & \multirow{2}{*}{37.00} & 1 \\
\hline 1-Butanamine & 109-73-9 & 73.14 & 0.97 & & & & & & 4 \\
\hline Diethyl malonate & $105-53-3$ & & 0.96 & $2.00 \mathrm{E}+04$ & Scenedesmus subspicatus & 72 & & \multirow{2}{*}{$\begin{array}{l}>800 \\
14.60\end{array}$} & 3 \\
\hline Glycidyl methacrylate & $106-91-2$ & & 0.96 & $5.00 \mathrm{E}+04$ & Selenastrum capricornutum & 72 & & & 1 \\
\hline 1,2,4-Benzenetricarboxylic acid & $528-44-9$ & & 0.95 & $2.10 \mathrm{E}+04$ & & & & 14.00 & 4 \\
\hline Methyl t-butyl ether & 1634-04-4 & 88.15 & 0.94 & & Scenedesmus subspicatus & 72 & & \multirow{2}{*}{$\begin{array}{l}>800 \\
10.70\end{array}$} & 3 \\
\hline Propanoic acid, 2-methyl- & $79-31-2$ & & 0.94 & $1.67 \mathrm{E}+02$ & Selenastrum capricornutum & 72 & & & 1 \\
\hline Propane, 2-nitro- & 79-46-9 & & 0.93 & $1.70 \mathrm{E}+04$ & algae & 72 & 1088.00 & \multirow{2}{*}{45.00} & 1 \\
\hline Methacrylic acid & $79-41-4$ & & 0.93 & $8.90 \mathrm{E}+04$ & Selenastrum capricornutum & 72 & & & 1 \\
\hline Dimethyl sulfide & $75-18-3$ & & 0.92 & $2.00 \mathrm{E}+04$ & $\begin{array}{l}\text { Pseudokirchneriella subcapitata } \\
\text { Pseudokirchneriella subcapitata }\end{array}$ & $\begin{array}{l}96 \\
72\end{array}$ & & $\begin{array}{l}19-26 \\
>113.7\end{array}$ & 1 \\
\hline $\begin{array}{l}\text { 2-Buten-1-ol, 3-methyl- } \\
\text { Methane, chloro- }\end{array}$ & $\begin{array}{l}556-82-1 \\
74-87-3\end{array}$ & 50.49 & $\begin{array}{l}0.91 \\
0.91\end{array}$ & $\begin{array}{c}1.70 \mathrm{E}+05 \\
4.80 \mathrm{E}+03-5.32 \mathrm{E}+03\end{array}$ & $\begin{array}{l}\text { Scenedesmus subspicatus } \\
\text { Scatia }\end{array}$ & 72 & & $>500$ & $\begin{array}{l}3 \\
4\end{array}$ \\
\hline Propanoic acid, 3-mercapto-, methyl ester & $2935-90-2$ & & 0.90 & $2.10 \mathrm{E}+04$ & Scenedesmus subspicatus & 72 & & & 1 \\
\hline Aniline & $62-53-3$ & & 0.90 & $3.50 \mathrm{E}+04$ & $\begin{array}{l}\text { Selenastrum capricornutum } \\
\text { Scenedesmus subspicatus }\end{array}$ & $\begin{array}{l}96 \\
72\end{array}$ & & & 1 \\
\hline Phenol, 2,4,6-trinitro- & $88-89-1$ & & 0.89 & $1.18 \mathrm{E}+04$ & $\begin{array}{l}\text { Scenedesmus subspicatus } \\
\text { Desmodesmus subspicatus }\end{array}$ & 72 & $>500$ & $\begin{array}{l}19.00 \\
>750\end{array}$ & 3 \\
\hline $\begin{array}{l}\text { Propanoyl chloride, 2,2-dimethyl- } \\
\text { n-Butanal }\end{array}$ & $\begin{array}{c}3282-30-2 \\
123-72-8\end{array}$ & $\begin{array}{l}120.58 \\
72.11\end{array}$ & $\begin{array}{l}0.89 \\
0.88\end{array}$ & $\begin{array}{l}3.48 \mathrm{E}+04 \\
1.18 \mathrm{E}+02\end{array}$ & Pseudokirchneriella subcapitata & 72 & & 878.00 & $\begin{array}{l}1 \\
4\end{array}$ \\
\hline n-Butanal & $123-72-8$ & 72.11 & 0.88 & $1.18 \mathrm{E}+02$ & Selenastrum capricornutum & 96 & 225.00 & \multirow{3}{*}{$>500$} & \\
\hline 1-Butanol & $71-36-3$ & & 0.88 & $7.70 \mathrm{E}+04$ & Scenedesmus subspicatus & 96 & & & 1 \\
\hline Butanamide, N-(2-methylphenyl)-3-oxo- & $93-68-5$ & & 0.85 & $3.00 \mathrm{E}+03$ & Selenastrum capricornutum & 72 & 383.00 & & 1 \\
\hline $\mathrm{o}$-Toluenesulfonamide & $88-19-7$ & & 0.84 & $1.60 \mathrm{E}+03$ & Selenastrum capricornutum & 72 & & \multirow[t]{2}{*}{170.00} & 1 \\
\hline D-Mannitol & $69-65-8$ & 171.23 & 0.84 & & & & & & 4 \\
\hline Carbon dioxide & $124-38-9$ & 44.01 & 0.83 & & & & & \multirow{2}{*}{23.00} & 4 \\
\hline p-Toluenesulfonamide & $70-55-3$ & & 0.82 & $3.20 \mathrm{E}+03$ & Selenastrum capricornutum & 72 & & & 1 \\
\hline Ethanol, 2-butoxy- & $111-76-2$ & & 0.80 & $5.00 \mathrm{E}+01$ & & & & \multirow{3}{*}{$>97$} & 4 \\
\hline 1,3-Benzenediol & $108-46-3$ & & 0.80 & 7.17E+05 & Pseudokirchneriello subcapitata & 72 & & & 3 \\
\hline Butanoic acid & $107-92-6$ & & 0.79 & miscible & & & & & 4 \\
\hline Propane, 1-nitro- & $108-03-2$ & & 0.79 & $1.40 \mathrm{E}+04$ & $\begin{array}{l}\text { Pseudokirchneriella subcapitata } \\
\text { Scenedesmus subspicatus }\end{array}$ & $\begin{array}{l}96 \\
96\end{array}$ & & \multirow{2}{*}{$\begin{array}{c}=>456 \\
98.00 \\
290.00\end{array}$} & 1 \\
\hline $\begin{array}{l}\text { 1-Propanol, 2-methyl- } \\
\text { Methanethiol }\end{array}$ & $\begin{array}{l}78-83-1 \\
74-93-1\end{array}$ & 74.12 & $\begin{array}{l}0.79 \\
0.78\end{array}$ & $\begin{array}{l}8.50 \mathrm{E}+04 \\
2.33 \mathrm{E}+01\end{array}$ & Selenastrum capricornutum & 48 & & & $\begin{array}{l}1 \\
4\end{array}$ \\
\hline Propanal, 2-methyl- & $78-84-2$ & 72.11 & 0.77 & $8.90 \mathrm{E}+04$ & Scenedesmus subspicatus & 72 & & \multirow{2}{*}{$\begin{array}{c}84.00 \\
2200.00\end{array}$} & 1 \\
\hline Hexane-1,6-diol & $629-11-8$ & 118.18 & 0.76 & miscible & Scenedesmus subspicatus & 72 & & & 1 \\
\hline Acetic acid, cyano- & $372-09-8$ & 1100.10 & 0.76 & $8.90 \mathrm{E}+05-1.00 \mathrm{E}+06$ & & 12 & & 200.00 & 4 \\
\hline Ethyl cyanoacetate & $105-56-6$ & & 0.76 & $2.00 \mathrm{E}+04$ & Scenedesmus subspicatus & 72 & & \multirow[t]{2}{*}{141.90} & 1 \\
\hline Ethane, 1,1-difluoro- & $75-37-6$ & 66.10 & 0.75 & $2.67 \mathrm{E}+03$ & & & & & 4 \\
\hline Methyl acrylate & $96-33-3$ & & 0.74 & $5.20 \mathrm{E}+04$ & $\begin{array}{l}\text { Selenastrum capricornutum } \\
\text { Scenedesmus subspicatus }\end{array}$ & $\begin{array}{l}72 \\
72\end{array}$ & $\begin{array}{c}3.55 \\
15.00\end{array}$ & & 1 \\
\hline Silanetriol, ethyl-, triacetate & $17689-77-9$ & 234.28 & 0.74 & $4.16 \mathrm{E}+04$ & Scenedesmus subspicatus & 72 & & 73.00 & 1 \\
\hline 2-Butanamine & $13952-84-6$ & 73.14 & 0.74 & & Desmodesmus subspicatus & 72 & & 2.03 & 1 \\
\hline Triethyl phosphite & $122-52-1$ & 166.16 & 0.74 & $1.50 \mathrm{E}+04$ & Scenedesmus subspicatus & 72 & & $>73.6$ & 3 \\
\hline Vinyl acetate & $108-05-4$ & 86.09 & 0.73 & & & & & & 4 \\
\hline Ethyl acetate & $141-78-6$ & & 0.73 & $8.30 \mathrm{E}+04$ & Scenedesmus subspicatus & 48 & & 5600.00 & 1 \\
\hline Ethylamine, N,N-dimethyl- & 598-56-1 & 73.14 & 0.70 & & Desmodesmus subspicatus & 72 & & 24.20 & 1 \\
\hline Acetoacetanilide & $102-01-2$ & & 0.70 & $8.38 \mathrm{E}+02-1.00 \mathrm{E}+04$ & Selenastrum capricornutum & 72 & 318.00 & & 1 \\
\hline Hydroperoxide, tert-butyl- & $75-91-2$ & 90.12 & 0.70 & $2.20 \mathrm{E}+01$ & & & & & 4 \\
\hline Mucochloric acid & $87-56-9$ & & 0.70 & $2.70 \mathrm{E}+04$ & Scenedesmus subspicatus & 72 & 62.00 & & 1 \\
\hline $\begin{array}{l}\text { 1-Propanaminium, N-(carboxymethyl)-N,N-dimethyl-3-[(1- } \\
\text { oxododecyl)amino]-, }\end{array}$ & 4292-10-8 & 343.53 & 0.69 & $1.76 \mathrm{E}+03$ & & & & & 4 \\
\hline 2-(Dimethylamino)ethyl acrylate & 2439-35-2 & & 0.68 & $2.40 \mathrm{E}+04$ & $\begin{array}{l}\text { Selenastrum capricornutum } \\
\text { Scenedesmus subspicatus }\end{array}$ & $\begin{array}{l}72 \\
72\end{array}$ & & $\begin{array}{l}>1.00 \\
0.88\end{array}$ & 1 \\
\hline 2-Oxepanone & 502-44-3 & & 0.68 & miscible & Scenedesmus subspicatus & 72 & & 1217.00 & 1 \\
\hline 2-Propenenitrile, 2-methyl- & $126-98-7$ & & 0.68 & $2.90 \mathrm{E}+04$ & Selenastrum capricornutum & 72 & & 25.30 & 1 \\
\hline
\end{tabular}


(Table S5. continued)

\begin{tabular}{|c|c|c|c|c|c|c|c|c|c|}
\hline Chemicals name & CAS Reg. no & $\begin{array}{c}\text { Molecular } \\
\text { weight } \\
\left(\mathrm{g} \mathrm{mol}^{-1}\right)\end{array}$ & $\log \mathrm{K}_{\mathrm{ow}}$ & Solubility $\left(\mathrm{mg} \mathrm{L}^{-1}\right)$ & Species & $\begin{array}{l}\text { Duration } \\
\text { (h) }\end{array}$ & $\begin{array}{c}\text { Measured } \\
E_{50} \\
\left(\mathrm{mg} \mathrm{L}^{-1}\right)\end{array}$ & $\begin{array}{c}\text { Nominal } \\
\mathrm{EC}_{50} \\
\left(\mathrm{mg} \mathrm{L}^{-1}\right)\end{array}$ & Classification \\
\hline Butane, 1,2-ероху- & $106-88-7$ & & 0.68 & $5.90 \mathrm{E}+04$ & Scenedesmus subspicatus & 72 & & $>500$ & 3 \\
\hline Diaminotoluene & $25376-45-8$ & & 0.66 & $1.39 \mathrm{E}+01$ & $\begin{array}{l}\text { Desmodesmus subspicatus } \\
\text { Desmodesmus subspicatus }\end{array}$ & $\begin{array}{l}72 \\
72\end{array}$ & 0.38 & 0.94 & 1 \\
\hline Toluene-3,4-diamine & 496-72-0 & & 0.66 & $1.39 \mathrm{E}+04$ & Desmodesmus subspicatus & 72 & & 0.94 & 1 \\
\hline 3-Buten-2-ol, 2-methyl- & $115-18-4$ & & 0.66 & $1.90 \mathrm{E}+05$ & Scenedesmus subspicatus & 72 & & $>500$ & 3 \\
\hline 2-Butanone, oxime & $96-29-7$ & & 0.65 & $1.10 \mathrm{E}+05$ & & & & & 4 \\
\hline $\begin{array}{l}\text { Benzenesulfonic acid, 2,2'-(1,2-ethenediyl)bis[5-[[4-[bis(2- } \\
\text { hydroxyethylaminol-6-(phenylamino)-1,3,5-triazin-2-yl]amino]- }\end{array}$ & 4404-43-7 & 960.95 & 0.65 & & & & & & 4 \\
\hline Carbonochloridic acid, ethyl ester & $541-41-3$ & 108.53 & 0.63 & 3.21E+04 & & & & & 4 \\
\hline Butan-2-ol & $78-92-2$ & & 0.61 & $4.50 \mathrm{E}+05$ & & & & & 4 \\
\hline Propanal & $123-38-6$ & 58.08 & 0.59 & $2.20 \mathrm{E}+05$ & Selenastrum capricornutum & 72 & & 58.00 & 1 \\
\hline Silane, dimethoxydimethyl- & $1112-39-6$ & & 0.59 & $6.80 \mathrm{E}+03$ & & & & & 4 \\
\hline Phthalonitrile & $91-15-6$ & & 0.58 & $5.60 \mathrm{E}+02$ & $\begin{array}{l}\text { Selenastrum capricornutum } \\
\text { Scenedesmus subspicatus }\end{array}$ & $\begin{array}{l}72 \\
72\end{array}$ & $\begin{array}{r}68.00 \\
421.00\end{array}$ & & 1 \\
\hline 2,4-Pentanediol, 2-methyl- & $107-41-5$ & & 0.58 & miscible & Selenastrum capricornutum & 72 & $>429$ & & 3 \\
\hline & & & & & Pseudokirchneriella subcapitata & 72 & 54.00 & & \\
\hline Diethylamine & $109-89-7$ & 73.14 & 0.58 & miscible & Pseudokirchneriella subcapitata & 96 & & 20.00 & 1 \\
\hline & & & & & Chlorella pyrenoidosa & 96 & & 56.00 & \\
\hline $\begin{array}{l}\text { Dibutyl phosphate } \\
\text { Ethanol, 2-(butoxyethoxy)- }\end{array}$ & $107-66-4$ & & 0.57 & $1.70 \mathrm{E}+04$ & Selenastrum capricornutum & 72 & & 92.00 & 1 \\
\hline Ethanol, 2-(butoxyethoxy)- & $112-34-5$ & 162.23 & 0.56 & miscible & Scenedesmus subspicatus & 96 & & $>100$ & 3 \\
\hline Silane, (3-chloropropyl)trimethoxy- & $2530-87-2$ & 199.00 & 0.56 & $6.50 \mathrm{E}+05$ & $\begin{array}{l}\text { Scenedesmus subspicatus } \\
\text { Selenastrum capricornutum }\end{array}$ & $\begin{array}{l}72 \\
72\end{array}$ & & $\begin{array}{r}>833 \\
555.00\end{array}$ & 1 \\
\hline Silane, trimethoxyphenyl- & 2996-92-1 & & 0.55 & $6.90 \mathrm{E}+04$ & Pseudokirchneriella subcapitata & 72 & & $>0.2$ & 3 \\
\hline 2-Butenal, 3-methyl- & $107-86-8$ & & 0.53 & $1.10 \mathrm{E}+05$ & Scenedesmus subspicatus & 72 & & 22.40 & 1 \\
\hline Triethyleneglycol, monobutylether & $143-22-6$ & & 0.51 & $1.00 \mathrm{E}+06$ & Scenedesmus subspicatus & 72 & & $>500$ & 3 \\
\hline Propanol, [(1-methyl-1,2-ethanediyl)bis(oxy)]bis- & $24800-44-0$ & & $\begin{array}{c}0.50- \\
0.60\end{array}$ & & Selenastrum capricornutum & 72 & & $>1000$ & 3 \\
\hline Hydroquinone & $123-31-9$ & & $\begin{array}{c}0.50- \\
0.61\end{array}$ & $7.30 \mathrm{E}+04$ & & & & & 4 \\
\hline 1,4-Butanediol & $110-63-4$ & & 0.50 & $1.00 \mathrm{E}+05$ & Selenastrum capricornutum & 72 & & $>1000$ & 3 \\
\hline Acrylic acid & 79-10-7 & & 0.46 & miscible & $\begin{array}{l}\text { Scenedesmus subspicatus } \\
\text { Scenedesmus subspicatus }\end{array}$ & $\begin{array}{l}72 \\
72\end{array}$ & $\begin{array}{l}0.13 \\
0.21\end{array}$ & & 1 \\
\hline Tetrahydrofuran & $109-99-9$ & 72.11 & 0.46 & & & & & & 4 \\
\hline Maleic acid & $110-16-7$ & 116.07 & 0.46 & 4.07E+05 & & & & & 4 \\
\hline Oxirane, chloromethyl- & $106-89-8$ & & 0.45 & $6.60 \mathrm{E}+04$ & 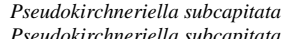 & $\begin{array}{l}72 \\
72\end{array}$ & 7.10 & $27.0-30.6$ & 1 \\
\hline Propionic acid, 3-mercapto- & $107-96-0$ & & 0.43 & $6.03 \mathrm{E}+05$ & $\begin{array}{l}\text { Pseudokirchneriella subcapitata } \\
\text { Pscila }\end{array}$ & 72 & 26.00 & $27.0=30.0$ & 1 \\
\hline 2-Hydroxyethyl methacrylate & 868-77-9 & & 0.42 & & Selenastrum capricornutum & 72 & & 345.00 & 1 \\
\hline 2-Furaldehyde & 98-01-1 & & 0.41 & $8.30 \mathrm{E}+04$ & & & & & 4 \\
\hline N-Vinyl-pyrrolidinone & $88-12-0$ & & 0.40 & & $\begin{array}{l}\text { Scenedesmus subspicatus } \\
\text { Scenedesmus subspicatus }\end{array}$ & $\begin{array}{l}72 \\
96\end{array}$ & & $\begin{array}{l}780.00 \\
770.00\end{array}$ & 1 \\
\hline Ether, methyl vinyl & $107-25-5$ & 58.08 & 0.40 & $1.71 \mathrm{E}+04$ & & & & & 4 \\
\hline tert-Butylamine & 75-64-9 & & 0.40 & & Pseudokirchnerella subcapitata & 96 & 16.00 & & 1 \\
\hline 2-Propanol, 1-methoxy-, acetate & $108-65-6$ & & 0.36 & $1.00 \mathrm{E}+05$ & Selenastrum capricornutum & 72 & & $>1,000$ & 3 \\
\hline Ethanol, 2-tert-butoxy- & $7580-85-0$ & 118.17 & 0.36 & $1.00 \mathrm{E}+05$ & Selenastrum capricornutum & 72 & & $>866$ & 3 \\
\hline Acrylic acid, monoester with 1,2-propanediol & $25584-83-2$ & 130.14 & 0.35 & $3.07 \mathrm{E}+05$ & Selenastrum capricornutum & 96 & & 6.67 & 1 \\
\hline Formaldehyde & $50-00-0$ & & 0.35 & & Scenedesmus quadricauda & 24 & & 14.70 & 1 \\
\hline 2,4-Pentanedione & $123-54-6$ & 100.12 & $\begin{array}{c}0.34- \\
0.40\end{array}$ & $1.66 \mathrm{E}+05$ & & & & & 4 \\
\hline Propane, 1-(allyloxy)-2,3-epoxy- & $106-92-3$ & & 0.34 & $1.28 \mathrm{E}+05$ & Pseudokirchneriella subcapitata & 72 & & $>79$ & 3 \\
\hline Propanal, 3-(methylthio)- & $3268-49-3$ & & 0.34 & $7.79 \mathrm{E}+04$ & Scenedesmus subspicatus & 72 & & 5.70 & 1 \\
\hline Propanoic acid, anhydride & $123-62-6$ & & 0.33 & & Scenedesmus subspicatus & 72 & & 45.80 & 1 \\
\hline Propionic acid & $79-09-4$ & & 0.33 & miscible & Scenedesmus subspicatus & 72 & & 45.80 & 1 \\
\hline 2-(2-Ethoxyethoxy)ethyl acetate & $112-15-2$ & 176.21 & 0.32 & $1.00 \mathrm{E}+06$ & & & & & 4 \\
\hline 3-Butyn-2-ol, 2-methyl- & $115-19-5$ & & 0.32 & miscible & Scenedesmus subspicatus & 72 & & $>500$ & 3 \\
\hline Propionitrile, 3-(triethoxysilyl)- & 919-31-3 & & 0.32 & $1.00 \mathrm{E}+06$ & Pseudokirchneriella subcapitata & 72 & $>3.6$ & & 3 \\
\hline Pentaerythritol & $115-77-5$ & & 0.30 & $2.50 \mathrm{E}+04$ & Selenastrum capricornutum & 72 & & $>1000$ & 3 \\
\hline Isocyanuric acid & $108-80-5$ & & 0.30 & $2.70 \mathrm{E}+03$ & Selenastrum capricornutum & 72 & & 620.00 & 1 \\
\hline 2-Butanone & $78-93-3$ & 72.11 & 0.29 & $2.76 \mathrm{E}+05$ & Scenedesmus quadricauda & 96 & & 1167.00 & 1 \\
\hline Acetic acid, mercapto- & $68-11-1$ & & 0.27 & $>1.00 \mathrm{E}+06$ & & & & & 4 \\
\hline
\end{tabular}


(Table S5. continued)

\begin{tabular}{|c|c|c|c|c|c|c|c|c|c|}
\hline Chemicals name & CAS Reg. no & $\begin{array}{c}\text { Molecular } \\
\text { weight } \\
\left(\mathrm{g} \mathrm{mol}^{-1}\right)\end{array}$ & $\log \mathrm{K}_{\mathrm{ow}}$ & Solubility $\left(\mathrm{mg} \mathrm{L}^{-1}\right)$ & Species & $\begin{array}{l}\text { Duration } \\
\text { (h) }\end{array}$ & $\begin{array}{c}\text { Measured } \\
\mathrm{EC}_{50} \\
\left(\mathrm{mg} \mathrm{L}^{-1}\right)\end{array}$ & $\begin{array}{c}\text { Nominal } \\
\mathrm{EC}_{50} \\
\left(\mathrm{mg} \mathrm{L}^{-1}\right)\end{array}$ & Classification \\
\hline 2-Propanamine & $75-31-0$ & & 0.26 & & Desmodesmus subspicatus & 72 & & 18.90 & 1 \\
\hline Ethyl acetoacetate & $141-97-9$ & & 0.25 & $1.25 \mathrm{E}+05$ & Scenedesmus subspicatus & 72 & & $>500$ & 3 \\
\hline Silanetriol, methyl-, triacetate & $4253-34-3$ & 220.00 & 0.25 & $9.10 \mathrm{E}+04$ & Scenedesmus subspicatus & 72 & 73.00 & & 1 \\
\hline 1-Propanol & $71-23-8$ & & 0.25 & & & & & & 4 \\
\hline Trimethylamine & $75-50-3$ & & 0.25 & 4.10E +05 & Desmodesmus subspicatus & 72 & & 150.00 & 1 \\
\hline 4-Piperidinol, 2,2,6,6-tetramethyl- & $2403-88-5$ & & 0.24 & $1.00 \mathrm{E}+05$ & $\begin{array}{l}\text { Selenastrum capricornutum } \\
\text { Selenastrum capricornutum }\end{array}$ & 72 & & $\begin{array}{l}155.00 \\
158.00\end{array}$ & 1 \\
\hline Dimethyl carbonate & $616-38-6$ & 90.08 & 0.23 & & & & & & 4 \\
\hline Chloroacetic acid & $79-11-8$ & 94.50 & 0.22 & $4.26 \mathrm{E}+03$ & Scenedesmus subspicatus & 48 & & 0.07 & 1 \\
\hline Ethanol, 2-(diethylamino)- & $100-37-8$ & & 0.21 & miscible & Scenedesmus subspicatus & 72 & & 44.00 & 1 \\
\hline Difluoromethane & $75-10-5$ & & 0.21 & $4.40 \mathrm{E}+03$ & & & & & 4 \\
\hline Acetin, tri- & $102-76-1$ & & 0.21 & $7.00 \mathrm{E}+04$ & Selenastrum capricornutum & 72 & & $>1,000$ & 3 \\
\hline Nitric acid & 7697-37-2 & 62.00 & 0.21 & very soluble & & & & & 4 \\
\hline Methyl acetate & $79-20-9$ & 74.08 & 0.18 & $2.50 \mathrm{E}+05-2.95 \mathrm{E}+05$ & Scenedesmus subspicatus & 72 & $>120$ & & 3 \\
\hline m-Phenylene-bis(methylamine) & $1477-55-0$ & & 0.18 & $1.00 \mathrm{E}+05$ & & & & & 4 \\
\hline Stannane, butyltrichloro- & $1118-46-3$ & & 0.18 & $1.00 \mathrm{E}+03-1.00 \mathrm{E}+05$ & Scenedesmus subspicatus & 72 & 0.31 & & 1 \\
\hline Butanol, 3-methoxy-3-methyl- & $56539-66-3$ & & 0.18 & $1.00 \mathrm{E}+05$ & Selenastrum capricornutum & 72 & & $>1,000$ & 3 \\
\hline Ethane, nitro- & $79-24-3$ & & 0.18 & $4.50 \mathrm{E}+04$ & Scenedesmus subspicatus & 96 & & 6.00 & 1 \\
\hline 2-Propen-1-ol & $107-18-6$ & 58.08 & 0.17 & miscible & Pseudokirchneriella subcapitata & 72 & 11.00 & & 1 \\
\hline Dimethyl sulfate & $77-78-1$ & & 0.16 & $2.80 \mathrm{E}+04$ & $\begin{array}{l}\text { Pseudokirchneriella subcapitata } \\
\text { Scenedesmus subspicatus }\end{array}$ & 72 & 7.80 & & 1 \\
\hline Formic acid, chloro-, methyl ester & $79-22-1$ & & 0.14 & $9.28 \mathrm{E}+05$ & Scenedesmus subspicatus & 72 & & 46.90 & 4 \\
\hline 1,3-Propanediol, 2,2-dimethyl- & $126-30-7$ & 104.15 & 0.12 & $1.90 \mathrm{E}+03$ & Selenastrum capricornutum & 72 & & $>1000$ & 3 \\
\hline 2H-Azepin-2-one, hexahydro- & $105-60-2$ & & 0.12 & $4.56 \mathrm{E}+06$ & $\begin{array}{l}\text { Scenedesmus subspicatus } \\
\text { Scenedesmus subspicatus }\end{array}$ & 72 & & 34.00 & 1 \\
\hline Adipic acid & $124-04-9$ & & 0.09 & $2.30 \mathrm{E}+04$ & $\begin{array}{l}\text { Scenedesmus subspicatus } \\
\text { Desmodesmus subspicatus }\end{array}$ & $\begin{array}{l}72 \\
72\end{array}$ & & $\begin{array}{c}4550.00 \\
31.30\end{array}$ & 1 \\
\hline Cyclohexanone & $108-94-1$ & 98.14 & 0.08 & $2.30 \mathrm{E}+04$ & & & & & 4 \\
\hline Benzenesulfonic acid, 4,4'-oxybis-, dihydrazide & $80-51-3$ & & 0.08 & $6.25 \mathrm{E}+01$ & $\begin{array}{l}\text { Pseudokirchneriella subcapitata } \\
\text { Pseudokirchneriella subcapitata }\end{array}$ & $\begin{array}{l}72 \\
72\end{array}$ & 3.00 & 6.70 & 1 \\
\hline Morpholine, 4-ethyl- & $100-74-3$ & & 0.08 & $3.03 \mathrm{E}+05$ & $\begin{array}{l}\text { Pseudokirchneriella subcapitata } \\
\text { Scenedesmus subspicatus }\end{array}$ & 72 & & $\begin{array}{c}>53 \\
58000\end{array}$ & 1 \\
\hline Ethanol, 2-propoxy- & $2807-30-9$ & & 0.08 & completely soluble & & & & & 4 \\
\hline Toluene, 2,4-diamine & 95-80-7 & & 0.07 & $3.80 \mathrm{E}+04$ & Selenastrum capricornutum & 96 & 9.54 & & 1 \\
\hline Phosphoryl trichloride & $10025-87-3$ & 153.33 & 0.06 & & Desmodesmus subspicatus & 72 & & 28.00 & 1 \\
\hline Oxirane, methyl- & $75-56-9$ & 58.08 & 0.06 & $3.95 \mathrm{E}+05-4.05 \mathrm{E}+05$ & Selenastrum capricornutum & 96 & & 240.00 & 1 \\
\hline 2-Propanol & $67-63-0$ & & 0.05 & $1.00 \mathrm{E}+06$ & & & & & 4 \\
\hline Phenol, 4-amino- & $123-30-8$ & & 0.04 & $1.57 \mathrm{E}+04$ & Pseudokirchneriella subcapitata & 72 & 0.15 & & 1 \\
\hline Silicic acid, (H4SiO4), tetraethyl ester & $78-10-4$ & & 0.04 & & Pseudokirchneriella subcapitata & 72 & & $>100$ & 3 \\
\hline Ethanol, 2-(1-methylethoxy)- & $109-59-1$ & & 0.04 & $1.00 \mathrm{E}+05$ & $\begin{array}{l}\text { Scenedesmus subspicatus } \\
\text { Pseudokirchneriella subcapitata }\end{array}$ & $\begin{array}{l}72 \\
72\end{array}$ & & $\begin{array}{l}>1039.3 \\
>1.000\end{array}$ & 3 \\
\hline $\begin{array}{l}\text { 1,2-Benzenedicarboxylic acid, bis(2-methoxyethyl) ester } \\
\text { (Di(methoxyethyl)phthalate) }\end{array}$ & $117-82-8$ & & 0.04 & $8.50 \mathrm{E}+00$ & & & & & 4 \\
\hline Methyl formate & $107-31-3$ & & 0.03 & $2.40 \mathrm{E}+04$ & $\begin{array}{l}\text { Desmodesmus subspicatus } \\
\text { Desmodesmus subspicatus }\end{array}$ & $\begin{array}{l}72 \\
72\end{array}$ & & $\begin{array}{l}1062.70 \\
1079.00\end{array}$ & 1 \\
\hline 1,6-Hexanediamine & $124-09-4$ & 116.24 & 0.02 & $8.00 \mathrm{E}+02$ & Selenastrum capricornutum & 96 & & 14.80 & 1 \\
\hline Toluene-2,3-diamine & $2687-25-4$ & 122.17 & 0.01 & $1.39 \mathrm{E}+04$ & & & & & 4 \\
\hline Propanol, 1(or 2)-(2-methoxymethylethoxy)- & $34590-94-8$ & & 0.01 & & Selenastrum capricornum & 72 & & $>969$ & 3 \\
\hline Chlorosulfuric acid & 7790-94-5 & 116.52 & 0.00 & & & & & & 4 \\
\hline Theophylline & $58-55-9$ & & -0.01 & $5.50 \mathrm{E}+03-8.30 \mathrm{E}+03$ & Scenedesmus subspicatus & 72 & & $>100$ & 3 \\
\hline Acrolein & $107-02-8$ & 56.06 & -0.01 & $2.06 \mathrm{E}+05-2.70 \mathrm{E}+05$ & Scenedesmus subspicatus & 72 & & 0.06 & 1 \\
\hline Glutaraldehyde & $111-30-8$ & & -0.01 & miscible & $\begin{array}{l}\text { Selenastrum capric } \\
\text { Scenedesmus supspicatus }\end{array}$ & 96 & & 3.90 & 1 \\
\hline Phosphonic acid, (1-hydroxyethylidene)bis- & 2809-21-4 & 206.03 & -0.01 & $6.90 \mathrm{E}+05$ & Selenastrum capricornutum & 96 & & 7.23 & 1 \\
\hline 1H-Imidazole & $288-32-4$ & & -0.02 & $6.63 \mathrm{E}+05$ & Scenedesmus subspicatus & 72 & & 133.00 & 1 \\
\hline 1,5-Pentanediol, 3-methyl- & $4457-71-0$ & & -0.03 & miscible & Selenastrum capricornutum & 72 & & $>1000$ & 3 \\
\hline Propanenitrile, 2-hydroxy-2-methyl- & $75-86-5$ & & -0.03 & miscible & Ankistrodesmus falcatus & 72 & & 1.25 & 1 \\
\hline Silane, ethenyltrimethoxy- & 2768-02-7 & 148.23 & -0.03 & $5.04 \mathrm{E}+05$ & $\begin{array}{l}\text { Scenedesmus subspicatus } \\
\text { Scenedesmus subspicatus }\end{array}$ & $\begin{array}{l}72 \\
72\end{array}$ & & $\begin{array}{l}>100 \\
>957\end{array}$ & 3 \\
\hline Dimethyl malonate & $108-59-8$ & 132.12 & -0.05 & $9.90 \mathrm{E}+04$ & $\begin{array}{l}\text { ceeneaesmus susppicatus } \\
\text { Scenedesmus subspicatus }\end{array}$ & $\begin{array}{l}12 \\
72 \\
\end{array}$ & & 386.00 & 1 \\
\hline
\end{tabular}


(Table S5. continued)

\begin{tabular}{|c|c|c|c|c|c|c|c|c|c|}
\hline Chemicals name & CAS Reg. no & $\begin{array}{c}\text { Molecular } \\
\text { weight } \\
\left(\mathrm{g} \mathrm{mol}^{-1}\right) \\
\end{array}$ & $\log \mathrm{K}_{\mathrm{ow}}$ & Solubility $\left(\mathrm{mg} \mathrm{L}^{-1}\right)$ & Species & $\begin{array}{l}\text { Duration } \\
\text { (h) }\end{array}$ & $\begin{array}{c}\text { Measured } \\
E C_{50} \\
\left(\mathrm{mg} \mathrm{L}^{-1}\right)\end{array}$ & $\begin{array}{l}\text { Nominal } \\
E_{50} \\
\left(\mathrm{mg} \mathrm{L}^{-1}\right)\end{array}$ & Classification \\
\hline Ethanol, 2-mercapto- & $60-24-2$ & 78.13 & -0.06 & miscible & $\begin{array}{l}\text { Desmodesmus subspicatus } \\
\text { Desmodesmus }\end{array}$ & 72 & $\begin{array}{l}19 \\
34\end{array}$ & & 1 \\
\hline Caffeine & $58-08-2$ & 194.19 & -0.09 & $2.00 \mathrm{E}+01$ & 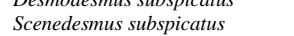 & 72 & & $>100$ & 3 \\
\hline 2-Furanmethanol, tetrahydro- & $97-99-4$ & 102.13 & -0.11 & $2.50 \mathrm{E}+05$ & Pseudokirchneriella subcapitata & 72 & & $>98.9$ & 3 \\
\hline Ethanamine & $75-04-7$ & & -0.13 & & & & & & 4 \\
\hline Acetaldehyde, oxime & $107-29-9$ & 59.07 & -0.13 & $>1.00 \mathrm{E}+04$ & & & & & 4 \\
\hline Diacetone alcohol & $123-42-2$ & 116.16 & -0.14 & $1.00 \mathrm{E}+05$ & Selenastrum capricornutum & 72 & & $>1000$ & 3 \\
\hline 2-Propanol, 1,1',1"-nitrilotri- & $122-20-3$ & 191.27 & -0.15 & miscible & Desmodesmus subspicatus & 72 & & 710.00 & 1 \\
\hline 2-Propenamide, 2-methyl- & $79-39-0$ & 85.10 & -0.15 & $1.00 \mathrm{E}+05$ & $\begin{array}{l}\text { Desmodesmus subspicatus } \\
\text { Selenastrum capricornutum }\end{array}$ & 72 & & $\begin{array}{l}>100 \\
>1000\end{array}$ & 3 \\
\hline Undecanoic acid, 11-amino- & 2432-99-7 & 201.31 & -0.16 & $8.00 \mathrm{E}+02$ & Pseudokirchneriella subcapitata & 72 & 53.00 & & 1 \\
\hline 2-Propanol, 1-[2-(2-methoxy-1-methylethoxy)-1-methylethoxy]- & 20324-33-8 & 206.28 & -0.20 & & & & & & 4 \\
\hline Ethanol, 2-(2-propoxyethoxy)- & 6881-94-3 & 148.20 & -0.20 & $1.00 \mathrm{E}+06$ & & & & & 4 \\
\hline 2-Hydroxyethyl acrylate & $818-61-1$ & 116.12 & -0.21 & & Selenastrum capricornutum & 72 & & 8.81 & 1 \\
\hline 2,3-Dibromosuccinic acid & $526-78-3$ & & -0.21 & $2.00 \mathrm{E}+04$ & & & & & 4 \\
\hline Acetone & 67-64-1 & 58.08 & -0.24 & $1.00 \mathrm{E}+06$ & & & & & 4 \\
\hline 1,4-Dioxane & $123-91-1$ & 88.11 & -0.27 & miscible & & & & & 4 \\
\hline Ethanol & $64-17-5$ & 46.07 & -0.31 & miscible & $\begin{array}{l}\text { Chlorella vulgaris } \\
\text { Selenastrum capricornutum }\end{array}$ & $\begin{array}{l}96 \\
96\end{array}$ & $\begin{array}{c}1000.00 \\
10000.00\end{array}$ & & 1 \\
\hline 1,4-Dicyanobutane & $111-69-3$ & 108.14 & -0.32 & $8.30 \mathrm{E}+04$ & Selenastrum capricornutum & 72 & & $>100$ & 3 \\
\hline Propanenitrile, 2-hydroxy- & $78-97-7$ & & -0.32 & infinite & Selenastrum capricornutum & 72 & & 0.14 & 1 \\
\hline Methane, nitro- & $75-52-5$ & & -0.33 & $1.11 \mathrm{E}+05$ & Scenedesmus subspicatus & 96 & 36.00 & & 1 \\
\hline Butanedioic acid, methylene- & $97-65-4$ & & -0.34 & & Scenedesmus subspicatus & 72 & 47.00 & & 1 \\
\hline 1,2-Butylene glycol & $584-03-2$ & 90.14 & -0.34 & $1.00 \mathrm{E}+05$ & Selenastrum capricornutum & 72 & & 10000.00 & 1 \\
\hline Acetonitrile & $75-05-8$ & 41.05 & -0.34 & $1.00 \mathrm{E}+06$ & & & & & 4 \\
\hline Oxydiethylene chloroformate & $106-75-2$ & & -0.34 & $3.00 \mathrm{E}+00$ & & & & & 4 \\
\hline Propane, 1-Amino-3-dimethylamino- & $109-55-7$ & & -0.35 & miscible & Scenedesmus subspicatus & 72 & & 56.20 & 1 \\
\hline Nicotinamide & $98-92-0$ & 122.13 & -0.38 & $6.91 \mathrm{E}+05-1.00 \mathrm{E}+06$ & Scenedesmus subspicatus, & 72 & $>1000$ & & 3 \\
\hline Dimethylamine & $124-40-3$ & 45.08 & -0.38 & . & 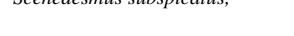 & 12 & 1000 & & 4 \\
\hline Propylamine, 3-methoxy- & $5332-73-0$ & 89.14 & $\begin{array}{l}-0.00 \\
-0.42\end{array}$ & & & & & & 4 \\
\hline 2-Propanol, 1-methoxy- & $107-98-2$ & 90.12 & -0.44 & $2.00 \mathrm{E}+05$ & & & & & 4 \\
\hline 2-Pyrrolidinone, 1-methyl- & $872-50-4$ & 99.13 & -0.46 & $1.00 \mathrm{E}+06$ & Scenedesmus subspicatus & 72 & $>500$ & & 3 \\
\hline Trimethyl phosphate & $512-56-1$ & & -0.46 & miscible & Selenastrum capricornutum & 72 & & $>1,000$ & 3 \\
\hline 1,3-Propanediol, 2-ethyl-2-(hydroxymethyl)- & $77-99-6$ & 134.20 & -0.47 & $>1.00 \mathrm{E}+05$ & Selenastrum capricornutum & 72 & & $>1000$ & 3 \\
\hline \multirow[t]{2}{*}{$\begin{array}{l}\text { Guanidine, cyano- } \\
\text { - }\end{array}$} & $461-58-5$ & 84.08 & -0.52 & $4.00 \mathrm{E}+04$ & Selenastrum capricornutum & 72 & 935.00 & & 1 \\
\hline & & & & & Selenastrum capricornutum & 120 & & 0.18 & \\
\hline \multirow[t]{2}{*}{ Peroxyacetic acid } & $79-21-0$ & 76.05 & -0.52 & $1.00 \mathrm{E}+06$ & Selenastrum capricornutum & 72 & & 0.86 & 1 \\
\hline & & & & & Selenastrum capricornutum & 72 & & $<1$ & \\
\hline \multirow{2}{*}{ Ethaneamine, 2'-oxibis[N,N-dimethyl-] } & $3033-62-3$ & 160.26 & -0.54 & $1.00 \mathrm{E}+06$ & Pseudokirchnerella subcapitata & 72 & 24.00 & & 1 \\
\hline & & & & & 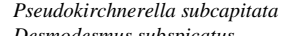 & 72 & 23.00 & & \\
\hline Formic acid & 64-18-6 & 46.03 & -0.54 & miscible & $\begin{array}{l}\text { Desmodesmus subspicatus } \\
\text { Scenedesmus subspicatus }\end{array}$ & $\begin{array}{l}72 \\
72\end{array}$ & & $\begin{array}{l}30.20 \\
26.90\end{array}$ & 1 \\
\hline Methanamine & $74-89-5$ & 31.06 & -0.57 & $1.00 \mathrm{E}+06$ & & & & & 4 \\
\hline Acetic anhydride & $108-24-7$ & 102.09 & -0.58 & $2.60 \mathrm{E}+04$ & & & & & 4 \\
\hline Nicotinic acid & $59-67-6$ & 123.11 & -0.59 & & Scenedesmus subspicatus & 72 & & 90.00 & 1 \\
\hline Lactic acid & $50-21-5$ & 90.10 & -0.62 & $8.76 \mathrm{E}+05$ & Pseudokirchneriella subcapitata & 70 & & 3500.00 & 1 \\
\hline 2-Propanol, 1,1'-oxydi- & $110-98-5$ & 134.18 & -0.64 & miscible & & & & & 4 \\
\hline $\mathrm{m}$-Toluenesulfonic acid, 6-amino- & $88-44-8$ & 187.22 & -0.67 & $6.00 \mathrm{E}+03$ & Selenastrum capricornutum & 72 & $>10$ & & 3 \\
\hline Silane, trimethoxymethyl- & $1185-55-3$ & & -0.67 & $1.00 \mathrm{E}+06$ & Pseudokirchneriella subcapitata & 72 & 210 & $>120$ & 3 \\
\hline DL-Pantoyl lactone & $79-50-5$ & 130.14 & -0.69 & $5.00 \mathrm{E}+02$ & Selenastrum capricornutum & 72 & & $>78$ & 3 \\
\hline Methyl acetoacetate & $105-45-3$ & & -0.69 & & & & & & 4 \\
\hline Ethanol, 2-(2-ethoxyethoxy)- & $111-90-0$ & 134.18 & -0.69 & $1.00 \mathrm{E}+06$ & & & & & 4 \\
\hline Silicic acid, ethyl ester & $11099-06-2$ & 106.15 & -0.72 & $1.00 \mathrm{E}+05$ & Scenedesmus subspicatus & 72 & & $>207$ & 3 \\
\hline 2-Butyne-1,4-diol & $110-65-6$ & 86.09 & -0.73 & $7.50 \mathrm{E}+05$ & Scenedesmus subspicatus & 72 & & 483.70 & 1 \\
\hline Methanol & $67-56-1$ & 32.04 & -0.74 & miscible & & & & & 4 \\
\hline Ethanol, 2,2'-thiodi- & $111-48-8$ & & -0.75 & miscible & Desmodesmus subspicatus & 72 & & $>500$ & 3 \\
\hline Phosphoric acid & 7664-38-2 & 98.00 & -0.77 & $5.48 \mathrm{E}+06$ & Pseudokirchneriella subcapitata & 72 & 77.90 & & 1 \\
\hline Thiophene, tetrahydro-, 1,1-dioxide & $126-33-0$ & 120.17 & -0.77 & $1.00 \mathrm{E}+05$ & Selenastrum capricornutum & 72 & & $>1000$ & 3 \\
\hline Acetamide, N,N-dimethyl- & $127-19-5$ & 87.12 & -0.77 & miscible & Scenedesmus subspicatus & 72 & & $>500$ & 3 \\
\hline
\end{tabular}


(Table S5. continued)

\begin{tabular}{|c|c|c|c|c|c|c|c|c|c|}
\hline Chemicals name & CAS Reg. no & $\begin{array}{c}\text { Molecular } \\
\text { weight } \\
\left(\mathrm{g} \mathrm{mol}^{-1}\right)\end{array}$ & $\log \mathrm{K}_{\mathrm{ow}}$ & Solubility $\left(\mathrm{mg} \mathrm{L}^{-1}\right)$ & Species & $\begin{array}{l}\text { Duration } \\
\text { (h) }\end{array}$ & $\begin{array}{c}\text { Measured } \\
\mathrm{EC}_{50} \\
\left(\mathrm{mg} \mathrm{L}^{-1}\right) \\
\end{array}$ & $\begin{array}{c}\text { Nominal } \\
\mathrm{EC}_{50} \\
\left(\mathrm{mg} \mathrm{L}^{-1}\right) \\
\end{array}$ & Classification \\
\hline Urea, 1,3-dimethyl- & $96-31-1$ & & -0.78 & $7.65 \mathrm{E}+05$ & Scenedesmus subspicatus & 72 & & $>500$ & 3 \\
\hline 2-Propanol, 1,1'-iminodi- & $110-97-4$ & 133.19 & -0.79 & $4.00 \mathrm{E}+05$ & $\begin{array}{l}\text { Scenedesmus subspicatus } \\
\text { Scenedesmus subspicatus }\end{array}$ & $\begin{array}{l}72 \\
72\end{array}$ & & $\begin{array}{l}339.00 \\
270.00\end{array}$ & 1 \\
\hline Formamide & $75-12-7$ & 45.04 & -0.82 & $1.00 \mathrm{E}+06$ & $\begin{array}{l}\text { Scenedesmus subspicatus } \\
\text { Sces }\end{array}$ & 96 & & $>500$ & 3 \\
\hline Formamide, N,N-dimethyl- & $68-12-2$ & 73.09 & -0.85 & miscible & $\begin{array}{l}\text { Scenedesmus subspicatus } \\
\text { Scenedesmus subspicatus }\end{array}$ & 96 & & $>1000$ & 3 \\
\hline & & & & & $\begin{array}{l}\text { Scenedesmus subspicatus } \\
\text { Sces }\end{array}$ & 72 & & 0.03 & \\
\hline Stannane, trichloromethyl- & $993-16-8$ & 240.10 & -0.90 & $1.04 \mathrm{E}+06$ & Ankistrodesmus falcatus & 24 & & 23.00 & 1 \\
\hline $\begin{array}{l}\text { Urea, N,N"-(2-methylpropylidene)bis- } \\
\text { Ethanol, 2-(methylamino)- }\end{array}$ & $\begin{array}{c}6104-30-9 \\
109-83-1\end{array}$ & 174.20 & $\begin{array}{l}-0.90 \\
-0.91\end{array}$ & $\begin{array}{c}3.00 \mathrm{E}+02-3.00 \mathrm{E}+03 \\
1.00 \mathrm{E}+06\end{array}$ & $\begin{array}{l}\text { Skeletonema costatum } \\
\text { Scenedesmus subspicatus } \\
\text { Desmodesmus subspicatus }\end{array}$ & $\begin{array}{l}96 \\
96 \\
72\end{array}$ & 28.10 & $\begin{array}{l}2.60 \\
>500\end{array}$ & $\begin{array}{l}3 \\
1\end{array}$ \\
\hline Silane, trimethoxy[3-(oxiranylmethoxy)propyl]- & $2530-83-8$ & 236.00 & -0.92 & $1.00 \mathrm{E}+06$ & $\begin{array}{l}\text { Selenastrum capricornutum } \\
\text { Scenedesmus subspicatus }\end{array}$ & $\begin{array}{l}96 \\
72\end{array}$ & & $\begin{array}{l}350.00 \\
255.00\end{array}$ & 1 \\
\hline Ethanol, 2-(dimethylamino)- & $108-01-0$ & 89.14 & -0.94 & miscible & $\begin{array}{l}\text { Scenedesmus sp } \\
\text { Spentus }\end{array}$ & 72 & & 35.00 & 1 \\
\hline 1,3-Propanediol, 2-(hydroxymethyl)-2-methyl- & $77-85-0$ & & -0.95 & $3.00 \mathrm{E}+05$ & Pseudokirchneriella subcapitata & 72 & & $>1000$ & 3 \\
\hline Triethylene glycol, monoethyl ether & $112-50-5$ & 178.20 & -0.96 & soluble & & & & & 4 \\
\hline 2-Propanol, 1-amino- & $78-96-6$ & 191.27 & -0.96 & & Desmodesmus subspicatus & 72 & & 32.70 & 1 \\
\hline Triethanolamine & $102-71-6$ & 149.19 & -1.00 & & $\begin{array}{l}\text { Scenedesmus subspicatus } \\
\text { Scenedesmus subspicatus }\end{array}$ & $\begin{array}{l}72 \\
96\end{array}$ & & $\begin{array}{l}216.00 \\
169.00\end{array}$ & 1 \\
\hline Ethanol, 2,2'-(methylimino)di- & $105-59-9$ & 119.16 & -1.08 & $1.00 \mathrm{E}+06$ & $\begin{array}{l}\text { Desmodesmus subspicatus } \\
\text { Skeletonema costatum }\end{array}$ & $\begin{array}{l}72 \\
72\end{array}$ & & $\begin{array}{l}175.70 \\
410.00\end{array}$ & 1 \\
\hline Bicyclo[2.2.2]octane, 1,4-diaza- & $280-57-9$ & & -1.13 & $6.10 \mathrm{E}+05$ & 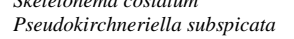 & 72 & & 180 & 1 \\
\hline Ethanol, 2-(2-methoxyethoxy)- & $111-77-3$ & 120.15 & -1.18 & & $\begin{array}{l}\text { Selenastrum cap } \\
\text { Scenedesmus subspicatus }\end{array}$ & $\begin{array}{l}96 \\
72\end{array}$ & & $\begin{array}{l}>1000 \\
>500\end{array}$ & 3 \\
\hline Dimethyl phosphonate & $868-85-9$ & & -1.20 & $>1.00 \mathrm{E}+5$ & Desmodesmus subspicatus & 72 & & $\geq 100$ & 3 \\
\hline Silane, trimethoxy- & $2487-90-3$ & & -1.22 & $1.00 \mathrm{E}+06$ & Pseudokirchneriello subcapitata & 72 & & $>100$ & 3 \\
\hline Piperazine & $110-85-0$ & & -1.24 & $1.50 \mathrm{E}+05$ & $\begin{array}{l}\text { Selenastrum capricornutum } \\
\text { Selenastrum capricornutum }\end{array}$ & $\begin{array}{l}72 \\
96\end{array}$ & & 34560 & 3 \\
\hline Diethylenetriamine & $111-40-0$ & 103.20 & -1.30 & miscible & $\begin{array}{l}\text { Scenedesmus subspicatus } \\
\text { Selenastrum cap }\end{array}$ & $\begin{array}{l}96 \\
72\end{array}$ & & $\begin{array}{c}592.00 \\
1164.00\end{array}$ & 1 \\
\hline Dimethyl sulfoxide & $67-68-5$ & 78.13 & -1.35 & $1.00 \mathrm{E}+06$ & & & & & 4 \\
\hline Ethylene glycol & $107-21-1$ & 62.07 & -1.36 & miscible & Selenastrum capriocornutum & 72 & non toxic & & 3 \\
\hline Melamine & $108-78-1$ & 126.12 & -1.37 & $3.10 \mathrm{E}+03$ & Scenedesmus & 96 & & 940.00 & 1 \\
\hline Ammonia & 7664-41-7 & 17.03 & -1.38 & $8.90 \mathrm{E}+05$ & & & & & 4 \\
\hline 7-Amino-4-hydroxy-2-naphthalenesulfonic acid & $87-02-5$ & & -1.39 & $5.00 \mathrm{E}+00$ & & & & & 4 \\
\hline Triethylene tetramine & $112-24-3$ & 146.23 & -1.40 & miscible & $\begin{array}{l}\text { Scenedesmus subspicatus } \\
\text { Selenastrum cappicornutum }\end{array}$ & $\begin{array}{l}72 \\
72\end{array}$ & & $\begin{array}{r}2.50 \\
2000\end{array}$ & 1 \\
\hline 1,2-Propanediol & $57-55-6$ & 76.09 & -1.41 & soluble & $\begin{array}{l}\text { Delenastrum capricornutum } \\
\text { Selentrum colum }\end{array}$ & 96 & & 19000.00 & 1 \\
\hline Ethanol, 2-(2-(2-methoxyethoxy)ethoxy)- & $112-35-6$ & 164.20 & -1.46 & soluble & Scenedesmus subspicatus & 72 & & $>500$ & 3 \\
\hline Ethanol, 2-((2-aminoethyl)amino)- & $111-41-1$ & 104.15 & -1.46 & unlimited & Scenedesmus subspicatus & 72 & & 354.00 & 1 \\
\hline Ethanol, 2,2'-oxybis- & $111-46-6$ & 106.12 & -1.47 & miscible & Skeletonema costatum & 72 & & $>1000$ & 3 \\
\hline Piperazine, 1-(2-aminoethyl)- & $140-31-8$ & 129.20 & -1.48 & miscible & Selenastrum capricornutum & 72 & & $>1000$ & 1 \\
\hline Propanol, oxybis- & $25265-71-8$ & & -1.49 & & Selenastrum capricornutum & 72 & & & 4 \\
\hline Stannane, tetrachloro- & $7646-78-8$ & 260.52 & -1.53 & water reactive & $\begin{array}{l}\text { Synechocystis aquatilis } \\
\text { Pseudokirchneriella subcapitata }\end{array}$ & $\begin{array}{l}96 \\
72\end{array}$ & & $\begin{array}{l}>23 \\
0.49\end{array}$ & 1 \\
\hline $\begin{array}{l}\text { Urea } \\
\text { Ure }\end{array}$ & $57-13-6$ & 60.06 & $\begin{array}{r}-1.59 \\
-1.63\end{array}$ & $\begin{array}{l}1.08 \mathrm{E}+06 \\
8.20 \mathrm{E}+05\end{array}$ & & & & & $\begin{array}{l}4 \\
3\end{array}$ \\
\hline $\begin{array}{l}\text { 1,3,5-Triazine-2,4,6(1H,3H,5H)-trione, 1,3,5-tris(2-hydroxyethyl)- } \\
\text { Glyoxal }\end{array}$ & $\begin{array}{l}839-90-7 \\
107-22-2\end{array}$ & 58.04 & $\begin{array}{r}-1.63 \\
-1.65\end{array}$ & $\begin{array}{c}8.20 \mathrm{E}+05 \\
\text { miscible }\end{array}$ & $\begin{array}{l}\text { Selenastrum capricornutum } \\
\text { Scenedesmus subspicatus }\end{array}$ & $\begin{array}{l}72 \\
72\end{array}$ & & $\begin{array}{l}>1000 \\
>250 \mathrm{mg}\end{array}$ & 3 \\
\hline Glyoxal & $101-22-2$ & 0.04 & -1.05 & miscible & $\begin{array}{l}\text { Scenedesmus subspicatus } \\
\text { Scenedsesmus subsicatus }\end{array}$ & $\begin{array}{l}96 \\
72\end{array}$ & 140.00 & $>500$ & \\
\hline 1,2,4-Butanetricarboxylic acid, 2-phosphono- & $37971-36-1$ & 270.13 & -1.66 & miscible & $\begin{array}{l}\text { Scenedesmus subspicatus } \\
\text { Scenedesmus subspicatus }\end{array}$ & 72 & 140.00 & $>1081$ & 1 \\
\hline 1,2-Ethanediamine, N-[3-(trimethoxysilyl)propyl]- & $1760-24-3$ & 222.00 & -1.67 & 1.00E-06 & Selenastrum capricornutum & 72 & & 8.80 & 1 \\
\hline 4,4'-Diaminostilbene-2,2'-disulfonic acid & $81-11-8$ & & -1.70 & $3.20 \mathrm{E}+01$ & Selenastrum capricornutum & 72 & & 76.00 & 2 \\
\hline Diazenedicarboxamide & $123-77-3$ & & -1.70 & $3.54 \mathrm{E}+04$ & Scenedesmus subspicatus & 72 & & $>36$ & 3 \\
\hline Citric acid & $77-92-9$ & 192.12 & -1.72 & $5.76 \mathrm{E}+05-7.71 \mathrm{E}+05$ & & & & & 4 \\
\hline 13-Tridecanol, 2,5,8,11-tetraoxa- & $23783-42-8$ & 208.26 & -1.73 & $1.00 \mathrm{E}+06$ & & & & & 4 \\
\hline Triethylene glycol & $112-27-6$ & 150.18 & -1.75 & completely soluble & & & & & 4 \\
\hline Glycerol & $56-81-5$ & 92.00 & -1.76 & miscible & & & & & 4 \\
\hline
\end{tabular}


(Table S5. continued)

\begin{tabular}{|c|c|c|c|c|c|c|c|c|c|}
\hline Chemicals name & CAS Reg. no & $\begin{array}{c}\text { Molecular } \\
\text { weight } \\
\left(\mathrm{g} \mathrm{mol}^{-1}\right)\end{array}$ & $\log \mathrm{K}_{\mathrm{ow}}$ & Solubility $\left(\mathrm{mg} \mathrm{L}^{-1}\right)$ & Species & $\begin{array}{l}\text { Duration } \\
\text { (h) }\end{array}$ & $\begin{array}{c}\text { Measured } \\
\mathrm{EC}_{50} \\
\left(\mathrm{mg} \mathrm{L}^{-1}\right) \\
\end{array}$ & $\begin{array}{c}\text { Nominal } \\
\mathrm{EC}_{50} \\
\left(\mathrm{mg} \mathrm{L}^{-1}\right) \\
\end{array}$ & Classification \\
\hline D-Gluconic acid & $526-95-4$ & 196.16 & -1.87 & $1.00 \mathrm{E}+03$ & Selenastrum capricornutum & 72 & & $>1000$ & 3 \\
\hline D-Glucono-1,5-lactone & $90-80-2$ & 178.14 & -1.98 & $5.90 \mathrm{E}+05$ & $\begin{array}{l}\text { Selenastrum capricornutum } \\
\text { Desmodesmus subspicatus }\end{array}$ & $\begin{array}{l}72 \\
72\end{array}$ & & $\begin{array}{l}>1000 \\
>100\end{array}$ & 3 \\
\hline $\begin{array}{l}\text { Dipentaerythritol } \\
\text { Tetraethylene glycol }\end{array}$ & $\begin{array}{l}126-58-9 \\
112-60-7\end{array}$ & $\begin{array}{l}254.00 \\
194.23\end{array}$ & $\begin{array}{l}-2.00 \\
-2.02\end{array}$ & $\begin{array}{l}3.00 \mathrm{E}+03 \\
\text { miscible }\end{array}$ & Scenedesmus subspicatus & 72 & & 100.00 & $\begin{array}{l}1 \\
4\end{array}$ \\
\hline Ethylenediamine & $107-15-3$ & 60.10 & -2.04 & $1.10 \mathrm{E}+05$ & Scenedesmus subspicatus & 48 & & $>100$ & 1 \\
\hline L-Ascorbic acid & $50-81-7$ & 176.12 & -2.05 & $3.30 \mathrm{E}+05$ & Selenastrum capricornutum & 72 & & 641.00 & 4 \\
\hline Stannane, dichlorodimethyl- & 753-73-1 & 219.69 & -2.18 & $8.23 \mathrm{E}+02$ & $\begin{array}{l}\text { Scenedesmus subspicatus } \\
\text { Scenedesmus obliquus }\end{array}$ & $\begin{array}{l}72 \\
96\end{array}$ & $\begin{array}{c}37.00 \\
1.20\end{array}$ & & 1 \\
\hline & & & & & Skeletonema costatum & 72 & & $>9.8$ & \\
\hline 2,2'-Iminodiethanol & $111-42-2$ & 105.14 & -2.18 & miscible & $\begin{array}{l}\text { Pseudokirchneriella subcapitata } \\
\text { Desmodesmus subspicatus }\end{array}$ & $\begin{array}{l}96 \\
72\end{array}$ & & $\begin{array}{l}2.10 \\
7.80\end{array}$ & 1 \\
\hline $\begin{array}{l}\text { 2-Imidazolidinone, 4,5-dihydroxy-1,3-bis } \\
\text { D-Glucose }\end{array}$ & $\begin{array}{c}1854-26-8 \\
50-99-7\end{array}$ & $\begin{array}{l}178.14 \\
180.16\end{array}$ & $\begin{array}{l}-2.20 \\
-2.20\end{array}$ & & Scenedesmus subspicatus & 72 & & 36.90 & $\begin{array}{l}1 \\
4\end{array}$ \\
\hline Sulfuric acid & 7664-93-9 & 98.07 & -2.21 & miscible & & & & & 4 \\
\hline Ethanol, 2-amino- & $141-43-5$ & 61.08 & -2.30 & & Pseudokirchneriella subcapitata & 72 & & 2.80 & 1 \\
\hline Pentaethylene glycol & $4792-15-8$ & 238.28 & -2.30 & $1.00 \mathrm{E}+06$ & & & & & 4 \\
\hline Trimethyl methylamine hydroxide & $75-59-2$ & 91.15 & -2.47 & $1.00 \mathrm{E}+06$ & Pseudokirchneriella subcapitata & 72 & & 96.00 & 1 \\
\hline Morpholine & $110-91-8$ & 87.12 & -2.55 & miscible & Pseudokirchneriella subcapitata & 72 & & 58.00 & 1 \\
\hline Maleic anhydride & $108-31-6$ & 98.06 & -2.61 & $4.00 \mathrm{E}+05$ & $\begin{array}{l}\text { Pseudokirchneriella subcapitata } \\
\text { Scenedesmus subspicata }\end{array}$ & $\begin{array}{l}72 \\
72\end{array}$ & & $\begin{array}{l}>150 \\
29.00\end{array}$ & 1 \\
\hline D-Glucitol & $50-70-4$ & 182.17 & -3.10 & & scenedesmus subspicata & & & & 4 \\
\hline Tetraethylenepentamine & $112-57-2$ & 189.30 & -3.16 & $1.00 \mathrm{E}+06$ & $\begin{array}{l}\text { Selenastrum capricornutum } \\
\text { Selenastrum capricornutum }\end{array}$ & $\begin{array}{l}72 \\
72\end{array}$ & & $\begin{array}{l}2.10 \\
6.80\end{array}$ & 1 \\
\hline Methanesulfinic acid, aminoimino- & $1758-73-2$ & 108.12 & -3.23 & $2.70 \mathrm{E}+04$ & Scenedesmus subspicatus & 72 & & 32.00 & 1 \\
\hline Syrups, hydrolyzed starch & 8029-43-4 & 180.16 & -3.24 & & non toxic & & & & 4 \\
\hline Glycine, N,N-1,3-propanediylbis[N-(carboxymethyl)- & $1939-36-2$ & 306.27 & -3.37 & & & & & & 4 \\
\hline Phosphonic acid, [nitrilotris(methylene)]tris- & $6419-19-8$ & 299.07 & -3.53 & $6.10 \mathrm{E}+05$ & Selenastrum & 96 & & 20.00 & 1 \\
\hline 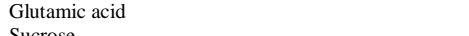 & $56-86-0$ & 147.13 & -3.69 & $8.64 \mathrm{E}+03$ & Pseudokirchneriella subcapitata & 72 & & 68.50 & 1 \\
\hline Sucrose & $57-50-1$ & 342.30 & -3.70 & & & & & & 4 \\
\hline Methenamine & $100-97-0$ & 140.19 & $\begin{array}{r}-4.15 \\
5.01\end{array}$ & $6.67 \mathrm{E}+05$ & Selenastrum capricornutum & 24 & & 0.30 & 1 \\
\hline E.D.T.A & $60-00-4$ & 292.30 & -5.01 & $4.00 \mathrm{E}+02$ & & & & & 4 \\
\hline $\begin{array}{l}\text { Phosphonic acid, [[(phosphonomethyl)imino]bis[2,1- } \\
\text { ethanediylnitrilobis(methylene)]]tetrakis- }\end{array}$ & $15827-60-8$ & 573.20 & -9.72 & $5.00 \mathrm{E}+05$ & $\begin{array}{l}\text { Selenastrum capricornutum } \\
\text { Selenastrum capricornutum } \\
\text { Selenastrum capricornutum }\end{array}$ & $\begin{array}{l}95 \\
95 \\
96\end{array}$ & & $\begin{array}{l}0.45 \\
>10 \\
0.83\end{array}$ & 1 \\
\hline $\begin{array}{l}\text { Maltodextrin } \\
\text { Hexafluorosilicic acid }\end{array}$ & $\begin{array}{r}9050-36-6 \\
16961-83-4\end{array}$ & $\begin{array}{l}342.30 \\
144.09\end{array}$ & & & & & & & $\begin{array}{l}4 \\
4 \\
\end{array}$ \\
\hline
\end{tabular}


Table S6. Summary of reported toxicity test results using invertebrates

\begin{tabular}{|c|c|c|c|c|c|c|c|c|c|}
\hline Chemicals name & CAS Reg. no & $\begin{array}{c}\text { Molecular } \\
\text { weight } \\
\left(\mathrm{g} \mathrm{mol}^{-1}\right)\end{array}$ & $\log \mathrm{K}_{\mathrm{ow}}$ & Solubility $\left(\mathrm{mg} \mathrm{L}^{-1}\right)$ & Species & $\begin{array}{l}\text { Duration } \\
\text { (h) }\end{array}$ & $\begin{array}{c}\text { Measured } \\
\mathrm{EC}_{50} \\
\left(\mathrm{mg} \mathrm{L}^{-1}\right)\end{array}$ & $\begin{array}{c}\text { Nominal } \\
E C_{50} \\
\left(\mathrm{mg} \mathrm{L}^{-1}\right)\end{array}$ & Classification \\
\hline C.I. Pigment Green 7 & $1328-53-6$ & 1127.19 & 17.40 & $7.00 \mathrm{E}-18-2.00 \mathrm{E}-16$ & $\begin{array}{l}\text { Daphnia magna } \\
\text { Daphnia magna }\end{array}$ & $\begin{array}{l}48 \\
48\end{array}$ & & $\begin{array}{r}>500 \\
153.60\end{array}$ & 2 \\
\hline Stannane, tetraoctyl- & $3590-84-9$ & 571.61 & 17.20 & 1.30E-12 & Daphnia magna & 24 & $<0.13$ & & 3 \\
\hline Di-n-octyltin bis(ethylhexylthioglycolate) & 15571-58-1 & 751.80 & 15.35 & $<1.00 \mathrm{E}-01$ & $\begin{array}{l}\text { Daphnia magna } \\
\text { Daphnia magna }\end{array}$ & $\begin{array}{l}48 \\
24\end{array}$ & $\begin{array}{l}0.17 \\
>0.06\end{array}$ & & 2 \\
\hline & & & & & Daphnia magna & 48 & 0.17 & & \\
\hline Diisooctyl 2,2'-[(dioctylstannylene)bis(thio)]diacetate & $26401-97-8$ & 751.80 & 15.35 & $<1.00 \mathrm{E}-01$ & Daphnia magna & 24 & $>0.38$ & & 2 \\
\hline Triisooctyl 2,2',2"-[(octylstannylidyne)tris(thio)]triacetate & $26401-86-5$ & 841.90 & 14.40 & $5.00 \mathrm{E}-01-2.70 \mathrm{E}+00$ & Daphnia magna & 48 & & 1.00 & 1 \\
\hline $\begin{array}{l}\text { 2-Ethylhexyl 10-ethyl-4-[[2-[(2-ethylhexyl)oxy]-2-oxoethyl]thio]-4-octyl-7- } \\
\text { oxo-8-oxa-3,5-dithia-4-stannatetradecanoate }\end{array}$ & 27107-89-7 & 841.90 & 14.10 & $5.00 \mathrm{E}-01-2.70 \mathrm{E}+00$ & Daphnia magna & 48 & & 1.00 & 1 \\
\hline Octadecanamide, N,N'-1,2-ethanediylbis- & $110-30-5$ & 593.04 & 13.98 & 1.10E-10 & & & & & 4 \\
\hline Ditridecyl phthalate & $119-06-2$ & 530.84 & 13.45 & $1.48 \mathrm{E}-09$ & & & & & 4 \\
\hline Octadecyl 3-(3,5-di-tert-butyl-4-hydroxyphenyl)propionate & 2082-79-3 & 530.88 & 13.40 & $2.85 \mathrm{E}-02$ & Daphnia magna & 24 & >solubility & & 3 \\
\hline Monobutyltin tris (2-ethylhexylmercaptoacetate) & 26864-37-9 & 785.79 & 12.45 & $6.00 \mathrm{E}-02-4.40 \mathrm{E}+00$ & & & & & 4 \\
\hline Bis-(2-Ethylhexyl) nonanedioate & $103-24-2$ & 412.66 & 11.90 & $4.00 \mathrm{E}-01$ & Daphnia magna & 48 & & >solubility & 3 \\
\hline Di-Undecyl phthalate & $3648-20-2$ & 474.00 & 11.49 & $1.61 \mathrm{E}-07$ & non toxic & & & & 3 \\
\hline $\begin{array}{l}\text { 8-Oxa-3,5-dithia-4-stannatetradecanoic acid, 4,4-dibutyl-10-ethyl-7-oxo-, 2- } \\
\text { ethylhexyl ester }\end{array}$ & $10584-98-2$ & 639.59 & 11.43 & $3.20 \mathrm{E}+02$ & $\begin{array}{l}\text { Daphnia magna } \\
\text { Daphnia magna }\end{array}$ & $\begin{array}{l}48 \\
48\end{array}$ & $>1.4$ & 0.04 & 1 \\
\hline $\begin{array}{l}\text { 8-Oxa-3,5-dithia-4-stannatetradecanoic acid, 10-ethyl-4-[[2-[(2- } \\
\text { ethylhexyl)oxy]-2-oxoethyl]thio]-4-methyl-7-oxo-, 2-ethylhexyl ester }\end{array}$ & 57583-34-3 & 743.71 & 10.98 & $1.80 \mathrm{E}+00-6.00 \mathrm{E}+00$ & non toxic & & & & 3 \\
\hline $\begin{array}{l}\text { Methacrylic acid, 2-methyl-, eicosyl ester } \\
\text { - Onde, }\end{array}$ & 45294-18-6 & 366.63 & 10.61 & 5.01E-06 & & & & & 4 \\
\hline Methacrylic acid, 2-methyl-, eicosyl ester & $26761-40-0$ & 446.68 & 10.36 & $1.04 \mathrm{E}-05$ & Daphnia magna & 48 & >solubility & & 3 \\
\hline 1,2-benzenedicarboxylic acid, di-2-propylheptyl ester & 53306-54-0 & 446.68 & 10.36 & $2.24 \mathrm{E}-06$ & non toxic & & & & 3 \\
\hline 2-Butanone, $\mathrm{O}, \mathrm{O}^{\prime}, \mathrm{O}^{\prime \prime}$-(ethenylsilylidyne)trioxime & 2224-33-1 & 313.48 & 10.19 & 3.00E-05 & & & & & 4 \\
\hline Docosanoic acid & $112-85-6$ & 340.60 & 9.91 & $1.60 \mathrm{E}-02$ & Daphnia magna & 48 & & $>5.00$ & 3 \\
\hline 2-Butanone, $\mathrm{O}, \mathrm{O}^{\prime}, \mathrm{O}$-(methylsilylidyne)trioxime & 22984-54-9 & 301.46 & 9.83 & $6.00 \mathrm{E}-05$ & Daphnia magna & 48 & & $>120$ & 3 \\
\hline 1-Docosanol & $661-19-8$ & 326.61 & 9.68 & $2.70 \mathrm{E}-03$ & & & & & 4 \\
\hline Octadecyl methacrylate & $32360-05-7$ & 338.58 & 9.62 & $5.49 \mathrm{E}-05$ & & & & & 4 \\
\hline Stannane, tetrabutyl- & $1461-25-2$ & 347.18 & 9.37 & $<1.00 \mathrm{E}-01$ & $\begin{array}{l}\text { Daphnia magna } \\
\text { Daphnia mogna }\end{array}$ & ${ }_{24}^{48}$ & 0.20 & 160 & 2 \\
\hline $\begin{array}{l}\text { Di(isononyl) phthalate } \\
\text { Stannane, dioctyloxo- }\end{array}$ & $\begin{array}{c}28553-12-0 \\
870-08-6\end{array}$ & $\begin{array}{l}418.62 \\
361.16\end{array}$ & $\begin{array}{l}9.37 \\
9.26\end{array}$ & $\begin{array}{l}2.32 \mathrm{E}-05 \\
6.57 \mathrm{E}-05\end{array}$ & non toxic & & & 1.00 & $\begin{array}{l}3 \\
4\end{array}$ \\
\hline 1-Octadecene & $112-88-9$ & 252.49 & 9.04 & $1.51 \mathrm{E}-04$ & Daphnia magna & 48 & & $\overrightarrow{\text { solubility }}$ & 3 \\
\hline Cyclohexasiloxane, dodecamethyl- & $540-97-6$ & 444.39 & 8.82 & $5.13 \mathrm{E}-03$ & & & & & 4 \\
\hline 1-Eicosanol & $629-96-9$ & 298.56 & 8.70 & $2.70 \mathrm{E}-03$ & & & & & 4 \\
\hline Hexadecyl methacrylate & $2495-27-4$ & 310.52 & 8.64 & 4.44E-04 & & & & & 4 \\
\hline Dimethylin bis[2-ethylhexylthioglycolate] & 57583-35-4 & 555.42 & 8.48 & $1.00 \mathrm{E}-01-4.90 \mathrm{E}+00$ & Daphnia magna & 48 & & 32.00 & 2 \\
\hline Bis(2-ethylhexyl) adipate & 103-23-1 & 370.64 & 8.39 & $3.20 \mathrm{E}-03$ & Daphnia magna & 48 & & >solubility & 3 \\
\hline Terephthalic acid, bis(2-ethylhexyl) ester & $6422-86-2$ & 390.57 & 8.39 & 4.00E-04 & Daphnia magna & 48 & & $\geq 0.0014$ & 3 \\
\hline 1-Octadecanol & $112-92-5$ & 270.50 & 8.22 & $1.10 \mathrm{E}-03$ & Daphnia magna & 48 & & 1700.00 & 2 \\
\hline Hexadecane & $544-76-3$ & 226.45 & 8.20 & $9.19 \mathrm{E}-04$ & non toxic & 40 & & 1700.00 & 3 \\
\hline Methacrylic acid, pentadecyl ester & $6140-74-5$ & 296.50 & 8.15 & $1.41 \mathrm{E}-03$ & & & & & 4 \\
\hline Pigment Yellow 13 & $5102-83-0$ & 685.62 & 8.11 & $3.50 \mathrm{E}-04$ & & & & >solubility & 3 \\
\hline 1-Hexadecen-3-ol, 3,7,11,15-tetramethyl- & $505-32-8$ & 296.54 & 8.10 & $5.80 \mathrm{E}+00$ & Daphnia magna & 48 & 0.13 & & 1 \\
\hline 1-Hexadecene & $629-73-2$ & 224.43 & 8.06 & $1.44 \mathrm{E}-03$ & Daphnia magna & 48 & & solubility & 3 \\
\hline 1-Dodecene & $112-41-4$ & 168.33 & 8.00 & $1.13 \mathrm{E}-01$ & Daphnia magna & 48 & & 480.00 & 2 \\
\hline Trisiloxane, 1,1,1,3,5,5,5-heptamethyl- & $1873-88-7$ & 222.51 & 7.84 & $2.00 \mathrm{E}-02$ & Daphnia mag & 48 & $>0.030$ & & 3 \\
\hline Trisiloxane, 1,1,1,5,5,5-hexamethyl-3,3-bis[(trimethylsilyl)oxy]- (M4Q) & $3555-47-3$ & 384.85 & 7.80 & $1.50 \mathrm{E}-04$ & & & & & 4 \\
\hline Cyclododecane, 1,2,5,6,9,10-hexabromo- & $3194-55-6$ & 641.70 & 7.74 & 3.40E-03 & Daphnia magna & 48 & & 0.00 & 2 \\
\hline Pentadecane & $629-62-9$ & 212.42 & 7.71 & $2.87 \mathrm{E}-03$ & non toxic & & & & 3 \\
\hline Tetradecyl methacrylate & $2549-53-3$ & 282.47 & 7.66 & 4.46E- 03 & & & & & 4 \\
\hline Octadecanamine, N,N-dimethyl-, N-oxide & 2571-88-2 & 313.57 & 7.62 & $3.18 \mathrm{E}-03$ & & & & & 4 \\
\hline $\begin{array}{l}\text { Butanamide, 2,2'-[(3,3'-dichloro[1,1'-biphenyl]-4,4'-diyl)bis(azo)]bis[N-(4- } \\
\text { chloro-2,5-dimethoxyphenyl)-3-oxo- }\end{array}$ & $5567-15-7$ & 818.50 & 7.54 & $8.10 \mathrm{E}-03$ & non toxic & & & & 3 \\
\hline 9-Octadecen-1-ol, (9Z)- & $143-28-2$ & 268.49 & 7.50 & 4.20E-02 & & & & & 4 \\
\hline Phenol, dodecyl- & $27193-86-8$ & 262.44 & 7.46 & & & & & & 4 \\
\hline
\end{tabular}


(Table S6. continued)

\begin{tabular}{|c|c|c|c|c|c|c|c|c|c|}
\hline Chemicals name & CAS Reg. no & $\begin{array}{c}\text { Molecular } \\
\text { weight } \\
\left(\mathrm{g} \mathrm{mol}^{-1}\right)\end{array}$ & $\log \mathrm{K}_{\mathrm{ow}}$ & Solubility $\left(\mathrm{mg} \mathrm{L}^{-1}\right)$ & Species & $\begin{array}{l}\text { Duration } \\
\text { (h) }\end{array}$ & $\begin{array}{c}\text { Measured } \\
\mathrm{EC}_{50} \\
\left(\mathrm{mg} \mathrm{L}^{-1}\right)\end{array}$ & $\begin{array}{c}\text { Nominal } \\
\text { EC } \text { So }_{50} \\
\left(\mathrm{mg} \mathrm{L}^{-1}\right)\end{array}$ & Classification \\
\hline \multirow[b]{2}{*}{ Benzene, undecyl- } & \multirow[b]{2}{*}{$6742-54-7$} & \multirow[b]{2}{*}{232.41} & \multirow[b]{2}{*}{7.45} & \multirow[b]{2}{*}{$4.10 \mathrm{E}-02$} & Daphnia magna & 48 & & 0.08 & \\
\hline & & & & & $\begin{array}{l}\text { Daphnia magna } \\
\text { Daphnia magna }\end{array}$ & 48 & & $\begin{array}{l}0.01 \\
0.01\end{array}$ & 1 \\
\hline Tetradecane & $629-59-4$ & 198.40 & 7.20 & $9.19 \mathrm{E}-03$ & $\begin{array}{l}\text { Dappnia magna } \\
\text { non toxic }\end{array}$ & 48 & & & 3 \\
\hline Tridecyl 2-methacrylate & $2495-25-2$ & 268.44 & 7.17 & & & & & & 4 \\
\hline Phenol, tetrapropylene & $57427-55-1$ & & 7.14 & & Daphnia magna & 48 & 0.02 & & 1 \\
\hline 1-Tetradecene & $1120-36-1$ & 196.38 & 7.08 & 4.00E-04 & Daphnia magna & 48 & & $=>1000$ & 3 \\
\hline \multirow[t]{2}{*}{ C.I. Pigment Yellow 12} & $6358-85-6$ & 629.51 & 7.05 & 4.00E-04 & Daphnia magna & 48 & & $\begin{array}{l}>\text { water } \\
\text { solubility }\end{array}$ & 3 \\
\hline & & & & & Daphnia pulex & 48 & & 0.13 & \\
\hline \multirow[t]{2}{*}{ Bis(2-ethylhexyl) phthalate } & $117-81-7$ & 390.57 & 7.00 & $1.30 \mathrm{E}+00$ & Daphnia carinata & 24 & & 0.33 & 1 \\
\hline & & & & & Daphnia magna & 48 & & 2.00 & \\
\hline Cyclotetrasiloxane, octamethyl- & $556-67-2$ & 296.62 & 6.79 & & & & & & 4 \\
\hline Tridecane & $629-50-5$ & 184.37 & 6.73 & & & & & & 4 \\
\hline 1-Hexadecanol & $36653-82-4$ & 242.45 & 6.73 & $1.30 \mathrm{E}-02$ & non toxic & & & & 3 \\
\hline Dodecyl methacrylate & $142-90-5$ & 254.42 & 6.68 & & & & & & 4 \\
\hline hexadecyldimethylamine $\mathrm{N}$-oxide & $7128-91-8$ & 285.52 & 6.64 & $3.20 \mathrm{E}-02$ & Daphnia magna & 48 & & 0.65 & 2 \\
\hline Trisiloxane, octamethyl- & $107-51-7$ & 236.54 & 6.60 & $3.40 \mathrm{E}-02$ & Daphnia magna & 48 & $>0.02$ & & 3 \\
\hline Methyl laurate & $111-82-0$ & 214.35 & 6.50 & $1.39 \mathrm{E}+00$ & Daphnia magna & 48 & 0.23 & & 1 \\
\hline Decanedioic acid, bis(2,2,6,6-tetramethyl-4-piperidinyl) ester & $52829-07-9$ & 480.74 & 6.50 & $1.88 \mathrm{E}+01$ & Daphnia magna & 24 & & 17.00 & 1 \\
\hline $\begin{array}{l}\text { Benzene, 1,1'-oxybis-, octabromo deriv. } \\
\text { B. }\end{array}$ & $32536-52-0$ & 801.38 & 6.29 & $5.00 \mathrm{E}-04$ & Daprina magna & 24 & & 17.00 & 4 \\
\hline Benzene, 1,1'-oxybis[2,3,4,5,6-pentabromo]- & $1163-19-5$ & 959.17 & 6.27 & $<1.00 \mathrm{E}-05$ & & & & & 4 \\
\hline p-Cresol, 6,6'-di-tert-butyl-2,2'-methylenedi- & $119-47-1$ & 340.51 & 6.25 & $2.00 \mathrm{E}-02$ & Daphnia magna & 48 & $>4.8$ & & 3 \\
\hline 1-Pentadecanol & $629-76-5$ & 228.42 & 6.24 & $1.02 \mathrm{E}-01$ & & & & & 4 \\
\hline Dodecane & $112-40-3$ & 170.34 & 6.23 & $1.10 \mathrm{E}-01$ & & & & & 4 \\
\hline 1-Dodecanethiol & $112-55-0$ & 202.40 & 6.20 & $1.00 \mathrm{E}+00$ & Daphnia magna & 48 & & not toxic & 3 \\
\hline \multirow[t]{2}{*}{ 1,1'-(1,1-dimethyl-3-methylene-1,3-propanediy)bisbenzene } & $6362-80-7$ & 236.36 & 6.20 & $2.30 \mathrm{E}-01$ & Daphnia magna & 48 & 0.06 & & 1 \\
\hline & & & & & Daphnia magna & 48 & 0.16 & & \\
\hline \multirow[t]{2}{*}{ tert-Dodecanethiol } & $25103-58-6$ & 202.40 & 6.20 & $2.50 \mathrm{E}-01$ & Daphnia magna & 24 & & 3.90 & 1 \\
\hline & & & & & Daphnia magna & 48 & & $>=0.25$ & \\
\hline Cyclohexane, 1,1'-methylenebis[4-isocyanato- & 5124-30-1 & 262.35 & 6.11 & $1.21 \mathrm{E}-01$ & Daphnia magna & 48 & $>8.3$ & & 3 \\
\hline Phenol, 2,6-bis(1,1-dimethylethyl)-4-(1-methylpropyl)- & $17540-75-9$ & 262.44 & 6.10 & $2.50 \mathrm{E}+00$ & & & & & 4 \\
\hline \multirow{4}{*}{$\begin{array}{l}\text { Dodecene } \\
\text { Ethanol, 2,2'-(octadecyloxidoimino)bis- }\end{array}$} & $25378-22-7$ & 168.33 & 6.10 & $1.25 \mathrm{E}-01$ & & & & & 4 \\
\hline & $14048-77-2$ & 373.63 & 6.08 & $2.90 \mathrm{E}-02$ & & & & & 4 \\
\hline & & & & & Daphnia magna & 48 & 0.19 & & \\
\hline & & & & & Daphnia magna & 48 & 0.09 & & \\
\hline \multirow[t]{3}{*}{ Phenol, nonyl- } & $25154-52-3$ & 220.36 & 5.99 & $1.57 \mathrm{E}+00$ & Daphnia magna & 48 & & 0.14 & 1 \\
\hline & (20) & 200.00 & ( & 1.50 & Ceriodaphnia dubia & 96 & 0.07 & & \\
\hline & & & & & Lumbriculus varieg & 96 & 0.27 & & \\
\hline 1,2,4-Benzenetricarboxylic acid, tris(2-ethylhexyl) ester & $3319-31-1$ & 546.79 & 5.94 & $1.30 \mathrm{E}-01$ & Daphnia magna & 48 & 0.21 & $>180$ & 3 \\
\hline $\begin{array}{l}2,2^{\prime}, 6,6 \text { '-Tetrabromo-4,4'-isopropylidenediphenol } \\
\text { lat }\end{array}$ & $79-94-7$ & 543.90 & 5.90 & $1.26 \mathrm{E}+00$ & Daphnia magna & 48 & 0.96 & 100 & 1 \\
\hline Ethanol, 2,2'-[(9Z)-9-octadecenyloxidoimino]bis- & 93962-62-0 & 371.61 & 5.86 & $4.50 \mathrm{E}-02$ & Daphnia & 48 & & $0.55,0.64$ & 2 \\
\hline $\begin{array}{l}\text { Triisobutylene } \\
\text { The }\end{array}$ & $7756-94-7$ & 168.33 & 5.85 & $1.90 \mathrm{E}-01$ & & 40 & & $0.00,0.04$ & 4 \\
\hline Stannane, dichlorodiocytyl- & $3542-36-7$ & 416.07 & 5.82 & $2.40 \mathrm{E}-01-2.80 \mathrm{E}-01$ & Daphnia magna & 48 & $>0.28$ & & 3 \\
\hline Benzene, dodecyl- & $123-01-3$ & 246.44 & 575 & $4.10 \mathrm{E}-02$ & $\begin{array}{l}\text { Daphhia magna } \\
\text { Daphnia magna }\end{array}$ & $\begin{array}{l}24 \\
48\end{array}$ & $\begin{array}{c}>0.005 \\
0.08\end{array}$ & & 2 \\
\hline 1-Tetradecanol & $112-72-1$ & 214.39 & 5.75 & $1.91 \mathrm{E}-01$ & $\begin{array}{l}\text { Daphnia magna } \\
\text { non toxic }\end{array}$ & & & & 3 \\
\hline $\mathrm{n}$-Undecane & $1120-21-4$ & 156.31 & 5.74 & $4.00 \mathrm{E}-03$ & Daphnia magna & 48 & 0.01 & & 2 \\
\hline Decene & 25339-53-1 & 140.27 & 5.70 & $2.10 \mathrm{E}-01$ & Daphnid & 48 & 0.01 & 0.16 & 1 \\
\hline Phosphine, triphenyl- & 603-35-0 & 262.29 & 5.69 & $1.65 \mathrm{E}-01$ & Daphnia magna & 48 & & $>5$ & 3 \\
\hline 1-Tetradecanamine, N,N-dimethyl-, N-oxide & $3332-27-2$ & 257.46 & 5.66 & 3.20E-01 & Daphnia magna & 48 & & 2.60 & 2 \\
\hline Nonane & $111-84-2$ & 128.26 & 5.65 & 4.06E-01 & Daphnia magna & 48 & 0.20 & & 1 \\
\hline Peroxide, bis(1-methyl-1-phenylethyl)- & $80-43-3$ & 270.37 & 5.60 & 4.30E-01 & Daphnia magna & 48 & $\begin{array}{l}>\text { water } \\
\text { solubility }\end{array}$ & & 3 \\
\hline Bumetrizole & $3896-11-5$ & 315.81 & 5.55 & $<1.00 \mathrm{E}+00$ & Daphnia magna & 48 & $\begin{array}{l}>\text { water } \\
\text { solubility }\end{array}$ & & 3 \\
\hline Metilox & $6386-38-5$ & 292.42 & $\begin{array}{l}5.50- \\
5.90\end{array}$ & $2.20 \mathrm{E}+00$ & Daphnia magna & 24 & & $\begin{array}{l}\text { >water } \\
\text { solubility }\end{array}$ & 3 \\
\hline 2-Ethylhexyl vinyl ether & $103-44-6$ & 156.27 & $\begin{array}{l}5.90 \\
5.50 \\
\end{array}$ & $1.80 \mathrm{E}+00$ & & & & & 4 \\
\hline
\end{tabular}


(Table S6. continued)

\begin{tabular}{|c|c|c|c|c|c|c|c|c|c|}
\hline Chemicals name & CAS Reg. no & $\begin{array}{c}\text { Molecular } \\
\text { weight } \\
\left(\mathrm{g} \mathrm{mol}^{-1}\right)\end{array}$ & $\log \mathrm{K}_{\mathrm{ow}}$ & Solubility $\left(\mathrm{mg} \mathrm{L}^{-1}\right)$ & Species & $\begin{array}{l}\text { Duration } \\
\text { (h) }\end{array}$ & $\begin{array}{c}\text { Measured } \\
\mathrm{ES}_{50} \\
\left(\mathrm{mg} \mathrm{L}^{-1}\right)\end{array}$ & $\begin{array}{c}\text { Nominal } \\
\text { EC } \\
\left(\mathrm{ES}_{50}\right. \\
\left(\mathrm{mg} \mathrm{L}^{-1}\right) \\
\end{array}$ & Classification \\
\hline $\begin{array}{l}\text { Peroxide, [1,3(or 1,4)-phenylenebis(1-methylethylidene)]bis[(1,1- } \\
\text { dimethylethyl) }\end{array}$ & 25155-25-3 & 338.49 & 5.50 & $4.00 \mathrm{E}-02$ & \multirow{3}{*}{ Daphnia magna } & \multirow{3}{*}{48} & \multirow{3}{*}{0.08} & & 4 \\
\hline 1-Dodecanamine, N,N-dimethyl- & $112-18-5$ & 213.41 & 5.47 & $1.00 \mathrm{E}+01$ & & & & & 1 \\
\hline $\begin{array}{l}\text { 1,3,5-Triazine-2,4,6(1H,3H,5H)-trione, 1,3,5-tris[[3,5-bis }(1,1- \\
\text { dimethylethyl)-4-hydroxyphenyl]methyl]- }\end{array}$ & $27676-62-6$ & 784.10 & 5.45 & $1.00 \mathrm{E}+01$ & & & & & 4 \\
\hline Ethanone, 1-(5,6,7,8-tetrahydro-3,5,5,6,8,8-hexamethyl-2-naphthalenyl)- & $1506-02-1$ & 258.41 & 5.40 & $1.22 \mathrm{E}+00$ & Daphnia magna & 72 & $>0.80$ & & 3 \\
\hline Ethanone, 1-(5,6,7,8-tetrahydro-3,5,5,6,8,8-hexamethyl-2-naphthalenyl)- & $21145-77-7$ & 258.41 & 5.40 & $1.22 \mathrm{E}+00$ & Daphnia magna & 72 & $>0.80$ & \multirow{3}{*}{0.25} & 3 \\
\hline Benzene, 1,4-dimethyl-2-(1-phenylethyl) & $6165-51-1$ & 210.31 & 5.39 & 9.60E-01 & \multirow[t]{2}{*}{ Daphnia magna } & \multirow[t]{2}{*}{48} & & & 1 \\
\hline 2,2',3,3'-Tetrachloro-4,4'-diaminodiphenylmethane (TCDAM) & 42240-73-3 & 322.02 & 5.39 & $2.27 \mathrm{E}-01-7.88 \mathrm{E}-01$ & & & & & 4 \\
\hline Disiloxane, 1,3-diethenyl-1,1,3,3-tetramethyl- & $2627-95-4$ & 186.40 & 5.36 & $2.07 \mathrm{E}-01$ & Daphnia magna & 48 & $>0.10$ & \multirow{4}{*}{$\begin{array}{c}\text { >solubility } \\
1.50\end{array}$} & 3 \\
\hline Stannane, dibutyloxo- & $818-08-6$ & 248.94 & 5.33 & $6.73 \mathrm{E}-01$ & Daphnia magna & 48 & & & 2 \\
\hline Cyclopenta[g]-2-benzopyran, 1,3,4,6,7,8-hexahydro-4,6,6,7,8,8-hexamethyl- & $1222-05-5$ & 258.41 & 5.30 & $1.75 \mathrm{E}+00$ & \multirow{3}{*}{ Daphnia magna } & \multirow{9}{*}{72} & 0.88 & & 1 \\
\hline Isodecyl methacrylate & 29964-84-9 & 226.36 & 5.28 & & & & & & 4 \\
\hline $\begin{array}{l}\text { 1(3H)-Isobenzofuranone, 6-(dimethylamino)-3,3-bis[4- } \\
\text { (dimethylamino)phenyl]- }\end{array}$ & $1552-42-7$ & 415.54 & 5.27 & $1.00 \mathrm{E}+01$ & & & & & 4 \\
\hline 1-Tridecanol & $112-70-9$ & 200.37 & 5.26 & $3.80 \mathrm{E}-01$ & \multirow[t]{3}{*}{ non toxic } & & & & 3 \\
\hline Triphenylmethyl chloride & $76-83-5$ & 278.78 & 5.25 & 5.35E-01 & & & & & 4 \\
\hline n-Decane & $124-18-5$ & 142.29 & 5.25 & & & & & & 4 \\
\hline Diphenylmethane diisocyanate (4,4'-M.D.I.) & $101-68-8$ & 250.25 & 5.22 & & \multirow[t]{3}{*}{ non toxic } & & & & 3 \\
\hline 2,2'-Diphenyl methane diisocyanate & $2536-05-2$ & 250.26 & 5.22 & & & & & & 4 \\
\hline 2,4'-Diphenyl methane diisocyanate & 5873-54-1 & 250.26 & 5.22 & & & & & & 4 \\
\hline Benzene, 1,1 '-methylenebis(isocyanato- & $26447-40-5$ & 250.26 & 5.22 & $8.29 \mathrm{E}-01$ & \multirow[t]{2}{*}{ Daphnia magna } & \multirow[t]{3}{*}{24} & & $>1,000$ & 3 \\
\hline Disiloxane, hexamethyl- & $107-46-0$ & 162.38 & 5.20 & $9.30 \mathrm{E}-01$ & & & & & 4 \\
\hline 1-Isotridecanol & 27458-92-0 & 200.37 & 5.19 & $5.24 \mathrm{E}+00$ & invertebrate & & $0.39-17.1$ & & 1 \\
\hline Octane & $111-65-9$ & 114.23 & 5.18 & $1.15 \mathrm{E}+00$ & \multirow[t]{2}{*}{ Daphnia magna } & \multirow[t]{2}{*}{48} & 0.30 & & 1 \\
\hline Ligroine & $8032-32-4$ & 114.23 & 5.18 & 1. HDLTOO & & & & & 4 \\
\hline 1-Decanol & $112-30-1$ & 158.29 & 5.13 & $3.95 \mathrm{E}+01$ & D. magna & \multirow{2}{*}{\multicolumn{2}{|c|}{48}} & 2.90 & 2 \\
\hline 1-Dodecanol & $112-53-8$ & 186.34 & 5.13 & $1.90 \mathrm{E}+00$ & Daphnids & & & 320.00 & 2 \\
\hline 1-Decene & $872-05-9$ & 140.27 & 5.12 & $1.15 \mathrm{E}-01$ & \multirow{2}{*}{$\begin{array}{l}\text { Daphnia magna } \\
\text { Daphnia magna }\end{array}$} & \multirow{2}{*}{\multicolumn{2}{|c|}{$\begin{array}{l}48 \\
48\end{array}$}} & 480.00 & 2 \\
\hline 2,6-Di-tert-butyl-p-cresol & $128-37-0$ & 220.36 & 5.10 & $1.10 \mathrm{E}+00$ & & & & >solubility & 3 \\
\hline $\begin{array}{l}\text { 2-Propenoic acid, 2-methyl-, (1R,2R,4R)-1,7,7-trimethylbicyclo[2.2.1] hept- } \\
\text { 2-yl ester, rel- }\end{array}$ & 7534-94-3 & 222.33 & 5.09 & $5.44 \mathrm{E}+00$ & Daphnia magna & 48 & & 1.10 & 1 \\
\hline Silane, dichlorodiphenyl- & $80-10-4$ & 253.20 & 5.06 & $2.80 \mathrm{E}+00$ & & & & & 4 \\
\hline Cyclopentadiene, hexachloro- & $77-47-4$ & 272.77 & 5.04 & $1.03 \mathrm{E}+00-1.25 \mathrm{E}+00$ & Daphnia magna & 48 & $\begin{array}{c}0.039- \\
0.052\end{array}$ & & 1 \\
\hline Pentaerythritol tetrakis[3-(3,5-di-tert-butyl-4-hydroxypheny & 6683-19-8 & 1177.67 & 5.00 & 1.00E-01 & non toxic & & & & 3 \\
\hline Pentene, 2,4,4-trimethyl- & $25167-70-8$ & 112.22 & 5.00 & $1.80 \mathrm{E}+00$ & Daphnia magna & 48 & 1.20 & & 1 \\
\hline 2-Ethylhexyl methacrylate & 688-84-6 & 198.31 & $\begin{array}{l}4.95- \\
5.59\end{array}$ & $1.60 \mathrm{E}+00$ & Daphnia magna & 48 & 4.56 & & 2 \\
\hline 2-Benzothiazolesulfenamide, N-cyclohexyl- & 95-33-0 & 264.41 & 4.93 & $3.20 \mathrm{E}-01$ & Daphnia magna & 48 & 0.79 & & 2 \\
\hline 5-tert-Butyl-2,4,6-trinitro-m-xylene (Musk xylene) & $81-15-2$ & 297.26 & 4.90 & $1.50 \mathrm{E}-01$ & Daphnia magna & 48 & & $>=0.15$ & 3 \\
\hline Neodecanoic acid, ethenyl ester & $51000-52-3$ & 198.30 & 4.90 & $5.90 \mathrm{E}+00$ & & & & & 4 \\
\hline Butyl benzyl phthalate & 85-68-7 & 312.36 & 4.84 & & & & & & 4 \\
\hline Perfluorooctanoic Acid & $335-67-1$ & 414.07 & 4.81 & $9.50 \mathrm{E}+03$ & Daphnia magna & 48 & 480.00 & & 1 \\
\hline 2-Benzothiazolesulfenamide, N,N-dicyclohexyl- & 4979-32-2 & 346.55 & 4.80 & $1.90 \mathrm{E}-03$ & $\begin{array}{l}\text { Daphnia magna } \\
\text { Daphnia magna }\end{array}$ & $\begin{array}{l}24 \\
24\end{array}$ & & $\begin{array}{l}>1000 \\
>0.0314\end{array}$ & 3 \\
\hline Benzenesulfonic acid, dodecyl- & $27176-87-0$ & 326.50 & 4.78 & 6.03E-01 & $\begin{array}{l}\text { Daphnia magna } \\
\text { Daphnia magna }\end{array}$ & $\begin{array}{l}24 \\
48\end{array}$ & & $\begin{array}{l}>0.0314 \\
3.5 \pm 1.0\end{array}$ & 2 \\
\hline Phenol, 5-chloro-2-(2,4-dichlorophenoxy)- & $3380-34-5$ & 289.54 & 4.76 & $1.00 \mathrm{E}+01$ & Daphnia magna & 48 & & 0.55 & 1 \\
\hline Cyclohexane, 5-isocyanato-1-(isocyanatomethyl)-1,3,3-trimethyl- & 4098-71-9 & 222.24 & 4.75 & $1.50 \mathrm{E}+01$ & Daphnia magna & 48 & & $\begin{array}{r}27.00 \\
35.00\end{array}$ & 2 \\
\hline p-Phenylenediamine, N-(1,3-dimethylbutyl)-N'-phenyl- & $793-24-8$ & 268.50 & 4.68 & $1.00 \mathrm{E}+00$ & $\begin{array}{l}\text { Daphnia magna } \\
\text { Daphnia magna }\end{array}$ & $\begin{array}{l}48 \\
48\end{array}$ & & $\begin{array}{l}35.00 \\
0.23\end{array}$ & 1 \\
\hline 1-Dodecanamine, N,N-dimethyl-, N-oxide & $1643-20-5$ & 229.41 & 4.67 & 3.13E+00 & $\begin{array}{l}\text { Daphnia magna } \\
\text { Daphnia magna }\end{array}$ & $\begin{array}{l}48 \\
48\end{array}$ & & 3.90 & 1 \\
\hline Amine oxides, cocoalkyldimethyl & 61788-90-7 & 229.41 & 4.67 & $3.13 \mathrm{E}+00$ & $\begin{array}{l}\text { Daphnia magna } \\
\text { Daphnia magna }\end{array}$ & $\begin{array}{l}48 \\
48\end{array}$ & 2.23 & 2.90 & 1 \\
\hline Benzothiazole disulfide & $120-78-5$ & 332.47 & 4.66 & & & & & & 4 \\
\hline Heptane & $142-82-5$ & 100.21 & 4.66 & $3.55 \mathrm{E}+00$ & Daphnia magna & 48 & & 3.90 & 2 \\
\hline 2-Naphthylisobutylether & $2173-57-1$ & 200.28 & 4.65 & $1.17 \mathrm{E}+00-6.91 \mathrm{E}+00$ & & & & & 4 \\
\hline Triphenyl phosphate & $115-86-6$ & 326.28 & 4.60 & $1.90 \mathrm{E}+00$ & $\begin{array}{l}\text { Daphnia magna } \\
\text { Daphnia magna }\end{array}$ & $\begin{array}{l}48 \\
48\end{array}$ & & $\begin{array}{l}1.00 \\
1.35\end{array}$ & 1 \\
\hline & & & & & Crangon crangon & 96 & 0.30 & & \\
\hline
\end{tabular}


(Table S6. continued)

\begin{tabular}{|c|c|c|c|c|c|c|c|c|c|}
\hline Chemicals name & CAS Reg. no & $\begin{array}{c}\text { Molecular } \\
\text { weight } \\
\left(\mathrm{g} \mathrm{mol}^{-1}\right)\end{array}$ & $\log \mathrm{K}_{\mathrm{ow}}$ & Solubility $\left(\mathrm{mg} \mathrm{L}^{-1}\right)$ & Species & $\begin{array}{l}\text { Duration } \\
\text { (h) }\end{array}$ & $\begin{array}{c}\text { Measured } \\
\mathrm{EC}_{50} \\
\left(\mathrm{mg} \mathrm{L}^{-1}\right) \\
\end{array}$ & $\begin{array}{c}\text { Nominal } \\
\mathrm{EC}_{50} \\
\left(\mathrm{mg} \mathrm{L}^{-1}\right) \\
\end{array}$ & Classification \\
\hline $\begin{array}{l}\text { Disperse Yellow-42 } \\
\text { tert-Nonyl mercaptan }\end{array}$ & $\begin{array}{c}5124-25-4 \\
25360-10-5\end{array}$ & & $\begin{array}{l}4.60 \\
4.59\end{array}$ & $\begin{array}{l}2.00 \mathrm{E}-01 \\
9.60 \mathrm{E}+00\end{array}$ & & & & & $\begin{array}{l}4 \\
4\end{array}$ \\
\hline Dibutyl phthalate & $84-74-2$ & 278.34 & 4.57 & $1.00 \mathrm{E}+01$ & $\begin{array}{l}\text { Daphnia magna } \\
\text { Daphnia magna }\end{array}$ & $\begin{array}{l}48 \\
48\end{array}$ & & $\begin{array}{l}3.20 \\
5.20\end{array}$ & 1 \\
\hline $\begin{array}{l}\text { Octene } \\
\text { Nonene }\end{array}$ & $\begin{array}{l}25377-83-7 \\
27215-95-8\end{array}$ & & 4.57 & $4.10 \mathrm{E}+00$ & & & & & 4 \\
\hline \multirow{3}{*}{$\begin{array}{l}\text { 2-Propenoic acid, 2-methyl-, 2-ethyl-2-[(2-methyl-1-oxo-2- } \\
\text { propenyl)oxy]methyl]-1,3-propanediyl ester }\end{array}$} & $27215-95-8$ & & 4.55 & $3.62 \mathrm{E}+00$ & & & & & 4 \\
\hline & $3290-92-4$ & 338.40 & 4.50 & $1.30 \mathrm{E}+00$ & & & & & 4 \\
\hline & & & & & Gammarus fasciatus & 48 & 0.80 & & \\
\hline \multirow[t]{4}{*}{ Phenol, 2,6-bis(1,1-dimethylethyl)- } & $128-39-2$ & 206.33 & 4.50 & $4.11 \mathrm{E}+00$ & $\begin{array}{l}\text { Gammarus fasciatus } \\
\text { Daphnia magna }\end{array}$ & $\begin{array}{l}72 \\
24\end{array}$ & 0.70 & 1.70 & 1 \\
\hline & & & & & Daphnia magna & 48 & 0.45 & & \\
\hline & & & & & Daphnia magna & 48 & 61.00 & & \\
\hline & & & & & Daphnia magna & 48 & & 27.00 & \\
\hline \multirow[t]{2}{*}{ Perfluorooctane sulfonic acid } & 1763-23-1 & 500.13 & 4.49 & $5.70 \mathrm{E}+02$ & Daphnia magna & 48 & & 210.00 & 1 \\
\hline & & & & & Daphnia magna & 48 & & 58.00 & \\
\hline Cyclotrisiloxane, hexamethyl- & $541-05-9$ & 222.46 & 4.47 & $1.60 \mathrm{E}+00$ & $\begin{array}{l}\text { Crassostrea virginica } \\
\text { Daphnia magna }\end{array}$ & $\begin{array}{l}48 \\
48\end{array}$ & $=>3.0$ & $>1.6$ & 3 \\
\hline Anthraquinone, 2-ethyl- & $84-51-5$ & & 4.37 & $4.05 \mathrm{E}-01$ & & & & 1.0 & 4 \\
\hline Dicyclohexylamine & 101-83-7 & 181.32 & 4.37 & $8.00 \mathrm{E}+02$ & $\begin{array}{l}\text { Daphnia magna } \\
\text { Daphnia magna }\end{array}$ & $\begin{array}{l}48 \\
48\end{array}$ & 8.00 & 70.1 & 1 \\
\hline 4-tert-Butyl-2,6-dimethyl-3,5-dinitroacetophenone (Musk ketone) & $81-14-1$ & 294.30 & 4.30 & $4.60 \mathrm{E}-01$ & Daphnia magna & 48 & & $>=0.46$ & 3 \\
\hline 2-Butanone, peroxide & $1338-23-4$ & 210.22 & 4.30 & $2.70 \mathrm{E}+00-1.40 \mathrm{E}+05$ & Daphnia magna & 48 & & 39.00 & 1 \\
\hline 1-Undecanol & $112-42-5$ & 172.31 & 4.28 & $8.00 \mathrm{E}+00$ & Nitocra spinipes & 96 & & $0.8-1.1$ & 1 \\
\hline \multirow{2}{*}{$\begin{array}{l}\text { 2,6-Dichlorotoluene } \\
\text { 1-Octanethiol }\end{array}$} & $118-69-4$ & 161.03 & 4.25 & $2.60 \mathrm{E}+01$ & Daphnia Magna & 24 & & 1.80 & 1 \\
\hline & $111-88-6$ & 146.29 & 4.21 & $1.98 \mathrm{E}-02$ & $\begin{array}{l}\text { Daphnia magna } \\
\text { Daphnia magna }\end{array}$ & $\begin{array}{l}48 \\
48\end{array}$ & 0.02 & $<0.42$ & 2 \\
\hline \multirow{4}{*}{$\begin{array}{l}\text { Dibutyl adipate } \\
\text { Camphene } \\
\text { m-Cresol, 6-tert-butyl- } \\
\text { Propanoic acid, 2-methyl-, 2,2-dimethyl-1-(1-methylethyl)-1,3-propanediyl } \\
\text { ester }\end{array}$} & $105-99-7$ & 258.35 & 4.17 & $3.50 \mathrm{E}+01$ & Daphnia magna & 24 & & 17.00 & 1 \\
\hline & $79-92-5$ & 136.23 & 4.12 & $4.20 \mathrm{E}+00$ & Daphnia magna & 48 & & 22.00 & 2 \\
\hline & $88-60-8$ & 164.24 & 4.11 & $4.20 \mathrm{E}+02$ & Daphnia magna & 48 & 2.77 & & 1 \\
\hline & $6846-50-0$ & 286.41 & 4.11 & $1.50 \mathrm{E}+01$ & Daphnia magna & 24 & & 300.00 & 2 \\
\hline Toluene, 2,4-dichloro- & $95-73-8$ & 161.03 & 4.10 & $2.50 \mathrm{E}+01$ & Daphnia magna & 24 & 19.00 & & 1 \\
\hline \multirow{2}{*}{$\begin{array}{l}\text { Pentane, 2,2,4-trimethyl- } \\
\text { Phenol, 2-(1,1-dimethylethyl)-4,6-dimethyl- }\end{array}$} & $540-84-1$ & 114.23 & 4.09 & $2.44 \mathrm{E}+00$ & & & & & 4 \\
\hline & 1879-09-0 & 178.27 & 4.08 & $1.50 \mathrm{E}+02$ & Daphnia magna & 24 & 5.60 & & 1 \\
\hline Benzene, 1,4-diethyl- & $105-05-5$ & 134.22 & 4.06 & $1.70 \mathrm{E}+01$ & & & & & 4 \\
\hline Benzene, 1,2,4-trichloro- & $120-82-1$ & 181.45 & 4.02 & $3.60 \mathrm{E}+01-4.80 \mathrm{E}+01$ & Daphnia magna & 48 & 3.39 & & 1 \\
\hline \multirow{2}{*}{$\begin{array}{l}\text { 1,1-Biphenyl } \\
\text { 3-Buten-2-one, 4-(2,6,6-trimethyl-1-cyclohexen-1-yl)- }\end{array}$} & $92-52-4$ & 154.21 & 4.01 & $7.48 \mathrm{E}+00$ & & & & & 4 \\
\hline & $79-77-6$ & 192.30 & 4.00 & $1.69 \mathrm{E}+02$ & Daphnia magna & 48 & 3.70 & & 1 \\
\hline \multirow{2}{*}{$\begin{array}{l}3,5,9 \text {-Undecatrien-2-one, 6,10-dimethyl- } \\
\text { Heptene }\end{array}$} & $141-10-6$ & 192.30 & 4.00 & $9.70 \mathrm{E}+01$ & Daphnia magna & 48 & 3.70 & & 1 \\
\hline & $25339-56-4$ & 172.00 & 3.99 & 1. $.82 \mathrm{E}+01$ & 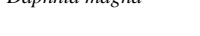 & 70 & . & & 4 \\
\hline \multirow{2}{*}{$\begin{array}{l}\text { Heptene } \\
\text { Isooctyl acrylate }\end{array}$} & 29590-42-9 & 184.20 & 3.93 & $1.24 \mathrm{E}+01$ & Daphnia magna & 48 & 0.40 & & 1 \\
\hline & $83-32-9$ & & 3.92 & $3.80 \mathrm{E}-03$ & & & & & 4 \\
\hline Acenaphthylene, 1,2-dihydro- & $80-07-9$ & 287.16 & 3.90 & $8.60 \mathrm{E}-01$ & Daphnia magna & 48 & & $>0.93$ & 3 \\
\hline & $95-31-8$ & 238.37 & 3.90 & $3.45 \mathrm{E}-01$ & Daphnia magna & 48 & 0.35 & & 1 \\
\hline $\begin{array}{l}\text { 2-Benzothiazolesulfeamide, N-(1,1-dimethylethyl)- } \\
\text { p-Phenylenediamine, N-(1-methylethyl)-N'-phenyl- }\end{array}$ & $101-72-4$ & 226.32 & 3.90 & $1.50 \mathrm{E}+01$ & Daphnia magna & 48 & & 1.10 & 1 \\
\hline $\begin{array}{l}\text { p-Phenylenediamine, N-(1-methylethyl)-N'-phenyl- } \\
\text { 2-Ethylhexyl acrylate }\end{array}$ & $103-11-7$ & 184.28 & 3.90 & $9.60 \mathrm{E}+00$ & Daphnia magna & 48 & 1.30 & & 1 \\
\hline \multirow{2}{*}{$\begin{array}{l}\text { Hexane } \\
\text { Linalylacetate }\end{array}$} & $110-54-3$ & 86.18 & 3.90 & & & & & & 4 \\
\hline & $115-95-7$ & 196.29 & 3.90 & $3.00 \mathrm{E}+01$ & Daphnia magna & 48 & 6.20 & & 1 \\
\hline $\begin{array}{l}\text { Linalylacetate } \\
\text { Phenol, 2,4,6-tribromo- }\end{array}$ & $118-79-6$ & 330.80 & 3.89 & $5.90 \mathrm{E}+01$ & $\begin{array}{l}\text { Daphnia magna } \\
\text { Daphnia magna }\end{array}$ & $\begin{array}{l}48 \\
48\end{array}$ & $\begin{array}{l}2.20 \\
0.26\end{array}$ & & 1 \\
\hline Naphthalene, methyl- & $1321-94-4$ & 142.20 & 3.87 & & & & & & 4 \\
\hline Naphthalene, 2-methyl- & $91-57-6$ & 142.20 & 3.86 & $2.46 \mathrm{E}+01$ & Daphnia magna & 48 & $1.42-2.99$ & & 1 \\
\hline 1H-Indene, 3a,4,7,7a-tetrahydro- & $3048-65-5$ & 120.19 & 3.83 & $3.74 \mathrm{E}+01$ & Daphnia magna & 48 & 0.73 & & 1 \\
\hline 2-Norbornene, 5-ethylidene- & $16219-75-3$ & 120.19 & 3.82 & $8.00 \mathrm{E}+01$ & $\begin{array}{l}\text { Daphnia magna } \\
\text { Daphnia magna }\end{array}$ & $\begin{array}{l}48 \\
48\end{array}$ & $\begin{array}{l}7.30 \\
3.34\end{array}$ & & 1 \\
\hline Aniline, 4-nitro-N-phenyl- & 836-30-6 & 214.00 & 3.82 & 4.10E+01 & $\begin{array}{l}\text { Daphnia magna } \\
\text { Daphnia magna }\end{array}$ & $\begin{array}{l}48 \\
48\end{array}$ & & 1.00 & 1 \\
\hline Silane, dichloromethylphenyl- & $149-74-6$ & & 3.80 & $7.85 \mathrm{E}+01$ & Daphnia magna & 48 & & 38.00 & 1 \\
\hline Naphthalene, 1,2,3,4-tetrahydro- & $119-64-2$ & 132.21 & 3.78 & $4.50 \mathrm{E}+01$ & Daphnia magna & 48 & & 9.50 & 1 \\
\hline 1-Pentanamine, N-pentyl- & $2050-92-2$ & 157.30 & 3.76 & $4.44 \mathrm{E}+02$ & Daphnia magna & 48 & 23.00 & & 1 \\
\hline
\end{tabular}


(Table S6. continued)

\begin{tabular}{|c|c|c|c|c|c|c|c|c|c|}
\hline Chemicals name & CAS Reg. no & $\begin{array}{c}\text { Molecular } \\
\text { weight } \\
\left(\mathrm{g} \mathrm{mol}^{-1}\right) \\
\end{array}$ & $\log \mathrm{K}_{\mathrm{ow}}$ & Solubility $\left(\mathrm{mg} \mathrm{L}^{-1}\right)$ & Species & $\begin{array}{l}\text { Duration } \\
\text { (h) }\end{array}$ & $\begin{array}{c}\text { Measured } \\
\mathrm{EC}_{50} \\
\left(\mathrm{mg} \mathrm{L}^{-1}\right) \\
\end{array}$ & $\begin{array}{c}\text { Nominal } \\
\mathrm{EC}_{50} \\
\left(\mathrm{mg} \mathrm{L}^{-1}\right) \\
\end{array}$ & Classification \\
\hline Anthraquinone, 1-amino- & $82-45-1$ & & 3.74 & $3.20 \mathrm{E}+01$ & Daphnia magna & 48 & & >solubility & 3 \\
\hline 2-Ethylhexyl acetate & $103-09-3$ & 172.27 & 3.74 & $3.90 \mathrm{E}+00$ & Daphnia magna & 48 & 22.90 & & 2 \\
\hline Naphthalene & $91-20-3$ & 128.18 & 3.73 & $3.00 \mathrm{E}+01$ & Daphnia magna & 24 & $1.42-2.99$ & & 1 \\
\hline Isodecanol & $25339-17-7$ & 158.29 & 3.71 & & & & & & 4 \\
\hline 1-Heptanol, 2-propyl- & $10042-59-8$ & 158.29 & 3.71 & & & & & & 4 \\
\hline Diphenyl tolyl phosphate & $26444-49-5$ & & 3.70 & $2.40 \mathrm{E}+00$ & Daphnia magna & 24 & 3.70 & & 2 \\
\hline Phenol, 4-(1,1,3,3-tetramethylbutyl)- & $140-66-9$ & 206.33 & 3.70 & $1.90 \mathrm{E}+01$ & Daphnia magna & 48 & & 0.27 & 1 \\
\hline Silane, triethoxyoctyl- & 2943-75-1 & & 3.70 & $1.30 \mathrm{E}-01$ & Daphnia magna & 48 & $>0.049$ & & 3 \\
\hline Decanamine, N,N-dimethyl-, N-oxide & $2605-79-0$ & 201.36 & 3.69 & $3.04 \mathrm{E}+01$ & & & & & 4 \\
\hline Amines, C10-16-alkyldimethyl, N-oxides & $70592-80-2$ & 201.36 & 3.69 & $3.04 \mathrm{E}+01$ & Daphnia magna & 96 & $0.8-1.2$ & & 1 \\
\hline Isooctyl mercaptoacetate & 25103-09-7 & & $3.68-$ & $1.06 \mathrm{E}+01$ & Daphnia magna & 48 & 0.39 & 024 & 1 \\
\hline N-(2-methylbutyl)-1-pentanamine & $61361-18-0$ & 157.30 & $\begin{array}{l}3.96 \\
3.68\end{array}$ & & Daphnia magna & & & 0.24 & 4 \\
\hline 4,4'-Methylenebis-(2-chlorobenzenamine) & $101-14-4$ & & 3.66 & $5.09 \mathrm{E}-01$ & Daphnia magna & 48 & $0.25-0.92$ & & 1 \\
\hline Cumene & $98-82-8$ & 120.19 & 3.66 & $7.50 \mathrm{E}+01$ & Dicanophorus forcipatus & 24 & 172.00 & & 2 \\
\hline $\begin{array}{l}\text { Cyclopropanecarboxylic acid, 3-(2,2-dichloroethenyl)-2,2-dimethyl-, methyl } \\
\text { ester }\end{array}$ & 61898-95-1 & & 3.66 & $5.30 \mathrm{E}+01$ & Daphnia magna & 48 & 7.04 & & 1 \\
\hline Benzene, 1,2,4-trimethyl- & $95-63-6$ & & 3.63 & & & & & & 4 \\
\hline Pyridine, 2,3,5,6-tetrachloro- & $2402-79-1$ & 216.87 & 3.63 & $2.94 \mathrm{E}+01-3.02 \mathrm{E}+01$ & $\begin{array}{l}\text { Daphnia magna } \\
\text { Daphnia magna }\end{array}$ & $\begin{array}{l}48 \\
48\end{array}$ & $\begin{aligned} & 1.93 \\
= & >2.67\end{aligned}$ & & 1 \\
\hline 2-Methyl-N-(2-methylbutyl)-1-butanamine & 27094-65-1 & 157.30 & 3.61 & & & & & & 4 \\
\hline Silane, trichlorophenyl- & $98-13-5$ & & 3.60 & $7.85 \mathrm{E}+02$ & Daphnia magna & 48 & & $>100$ & 3 \\
\hline Phenol, 2-sec-butyl-4,6-dinitro- & $88-85-7$ & 240.21 & 3.57 & $3.45 \mathrm{E}+01$ & $\begin{array}{l}\text { Daphnia magna } \\
\text { Daphnia magna } \\
\text { Daphnia magna }\end{array}$ & $\begin{array}{l}48 \\
48 \\
48\end{array}$ & 0.40 & $\begin{array}{l}0.24 \\
0.74\end{array}$ & 1 \\
\hline 1-Octene & $111-66-0$ & 112.21 & $\begin{array}{l}3.50- \\
460\end{array}$ & $4.10 \mathrm{E}+00$ & $\begin{array}{l}\text { Daphnia magna } \\
\text { Daphnia magna }\end{array}$ & 48 & & 67.00 & 1 \\
\hline 2,6-Naphthalenedicarboxylic acid, dimethyl ester & 840-65-3 & & 3.50 & $1.50 \mathrm{E}-01$ & Daphnia magna & 48 & $>0.1$ & 3.2-10 & 3 \\
\hline $\begin{array}{l}\text { Formic acid, chloro-, 2-ethylhexyl ester } \\
\text { Tributyl phoshate }\end{array}$ & $24468-13-1$ & 192.69 & 3.50 & $4.90 \mathrm{E}+01$ & Daphnia maong & 48 & & & 4 \\
\hline Tributyl phosphate & 126-73-8 & 266.31 & 3.50 & $4.00 \mathrm{E}+02$ & $\begin{array}{l}\text { Daphnia magna } \\
\text { Daphnia magna }\end{array}$ & $\begin{array}{l}48 \\
48\end{array}$ & & $\begin{array}{c}2.6-9 \\
4.00\end{array}$ & 1 \\
\hline Phenol, 2-sec-butyl- & $89-72-5$ & 150.22 & 3.49 & $1.52 \mathrm{E}+03$ & $\begin{array}{l}\text { Dappnna magna } \\
\text { Daphnia magna }\end{array}$ & 48 & & 3.70 & 1 \\
\hline Benzenesulfonyl chloride, 4-methyl- & $98-59-9$ & 190.65 & 3.49 & $5.12 \mathrm{E}+01$ & Daphnia magna & 48 & & 70.00 & 2 \\
\hline $\begin{array}{l}\text { Benzene, (1-methylethenyl)- } \\
\text { Propanoic acid, 2-methyl-, monoester with 2,2,4-trimethyl-1,3-pentanediol }\end{array}$ & $\begin{array}{c}98-83-9 \\
25265-77-4\end{array}$ & 216.32 & $\begin{array}{l}3.48 \\
3.47\end{array}$ & $\begin{array}{l}1.00 \mathrm{E}+02 \\
8.58 \mathrm{E}+02\end{array}$ & $\begin{array}{l}\text { Daphnia Magna } \\
\text { Daphnia magna }\end{array}$ & $\begin{array}{l}48 \\
48\end{array}$ & & $\begin{array}{l}54.00 \\
>=95\end{array}$ & $\begin{array}{l}1 \\
3\end{array}$ \\
\hline Benzene, 1,4-dichloro- & $106-46-7$ & 147.00 & 3.44 & $6.00 \mathrm{E}+01-7.00 \mathrm{E}+01$ & $\begin{array}{l}\text { Daphnia magna } \\
\text { Daphnia magna }\end{array}$ & $\begin{array}{l}48 \\
24\end{array}$ & $\begin{array}{l}0.70 \\
1.60\end{array}$ & & 1 \\
\hline Cyclohexane & $110-82-7$ & 84.16 & 3.44 & $5.80 \mathrm{E}+01$ & $\begin{array}{l}\text { Daphnia magna } \\
\text { Daphnia magna }\end{array}$ & $\begin{array}{l}48 \\
48\end{array}$ & 0.90 & 3.78 & 1 \\
\hline Benzoyl peroxide & $94-36-0$ & 242.24 & 3.43 & $9.10 \mathrm{E}+00$ & Daphnia Magna & 48 & 0.07 & & 1 \\
\hline Methacrylic acid, 3-(trichlorosilyl)propyl ester & $7351-61-3$ & & 3.43 & $9.87 \mathrm{E}+01$ & Daphnia Magna & 48 & 2.91 & & 4 \\
\hline Toluene, 2-chloro- & $95-49-8$ & 126.58 & 3.42 & $4.70 \mathrm{E}+01$ & Daphnia magna & $\begin{array}{l}24 \\
96\end{array}$ & & $\begin{array}{c}20.00 \\
40-50\end{array}$ & 1 \\
\hline $\begin{array}{l}\text { Benzene, 1,3,5-trimethyl- } \\
\text { 1-Hexanol, 3,5,5-trimethyl- }\end{array}$ & $\begin{array}{l}108-67-8 \\
3452-97-9\end{array}$ & 120.20 & $\begin{array}{l}3.42 \\
3.42\end{array}$ & $\begin{array}{l}4.00 \mathrm{E}+01-7.50 \mathrm{E}+01 \\
4.50 \mathrm{E}+02\end{array}$ & Daphnia magna & 48 & 6.77 & & $\begin{array}{l}4 \\
1\end{array}$ \\
\hline 2-Hydroxy-3-napthoic acid & $92-70-6$ & 188.18 & $\begin{array}{l}3.40- \\
3.59\end{array}$ & $4.74 \mathrm{E}+02$ & $\begin{array}{l}\text { Daphnia magna } \\
\text { Daphnia magna }\end{array}$ & $\begin{array}{l}24 \\
48\end{array}$ & $\begin{array}{r}137.03 \\
32.90\end{array}$ & & 1 \\
\hline Bisphenol A & $80-05-7$ & 228.29 & 3.40 & $3.00 \mathrm{E}+02$ & $\begin{array}{l}\text { Daphnia magna } \\
\text { Daphnia magna }\end{array}$ & $\begin{array}{l}48 \\
48\end{array}$ & 10.20 & 3.90 & 1 \\
\hline & & & & & Daphnia magna & 48 & & 2.40 & \\
\hline Benzene, 1,2-dichloro- & $95-50-1$ & 147.00 & 3.40 & $1.56 \mathrm{E}+02$ & Daphnia magna & 48 & & 25.70 & 1 \\
\hline DL-menthol & $1490-04-6$ & & 3.40 & $5.08 \mathrm{E}+02$ & Daphnia magna & 48 & & 16.00 & 4 \\
\hline L-Menthol & $2216-51-5$ & 156.27 & 3.40 & $4.31 \mathrm{E}+02$ & $\begin{array}{l}\text { Daphnia magna } \\
\text { Daphnia magna }\end{array}$ & $\begin{array}{l}48 \\
24\end{array}$ & 26.60 & 37.70 & 1 \\
\hline (+)-Menthol & $15356-60-2$ & 156.27 & 3.40 & $4.20 \mathrm{E}+02-5.00 \mathrm{E}+02$ & & & & & 4 \\
\hline Ethene, tetrachloro- & $127-18-4$ & 165.83 & 3.40 & $1.60 \mathrm{E}+02$ & $\begin{array}{l}\text { Daphnia magna } \\
\text { Daphnia magna }\end{array}$ & $\begin{array}{l}48 \\
48\end{array}$ & $\begin{array}{l}22.00 \\
8.50\end{array}$ & & 1 \\
\hline Hexene & $25264-93-1$ & & 3.39 & $5.00 \mathrm{E}+01$ & & & & & 4 \\
\hline
\end{tabular}




\section{(Table S6. continued)}

\begin{tabular}{|c|c|c|c|c|c|c|c|c|c|}
\hline Chemicals name & CAS Reg. no & $\begin{array}{c}\text { Molecular } \\
\text { weight } \\
\left(\mathrm{g} \mathrm{mol}-I^{-1}\right) \\
\end{array}$ & $\log \mathrm{K}_{\mathrm{ow}}$ & Solubility $\left(\mathrm{mg} \mathrm{L}^{-1}\right)$ & Species & $\begin{array}{l}\text { Duration } \\
\text { (h) }\end{array}$ & $\begin{array}{c}\text { Measured } \\
\mathrm{EC}_{50} \\
\left(\mathrm{mg} \mathrm{L}^{-1}\right) \\
\end{array}$ & $\begin{array}{c}\text { Nominal } \\
\text { EC } \\
\left(\mathrm{CE}_{50}^{-1}\right) \\
\left(\mathrm{mg} \mathrm{L}^{-1}\right) \\
\end{array}$ & Classification \\
\hline 1-Hexene & $592-41-6$ & 84.16 & 3.39 & $5.00 \mathrm{E}-02$ & Daphnia magna & 48 & & 230.00 & 2 \\
\hline Pentane & $109-66-0$ & & 3.39 & $3.85 \mathrm{E}+01$ & Daphnia magna & 48 & 2.70 & 2000 & 1 \\
\hline Dibutyl maleate & $105-76-0$ & & 3.38 & $1.73 \mathrm{E}+02$ & Daphnia magna & 24 & & 45.00 & 1 \\
\hline Butane, 1,1'-oxybis- & $142-96-1$ & 130.23 & 3.35 & $1.13 \mathrm{E}+02$ & Daphnia magna & 48 & & 26.00 & 1 \\
\hline Neodecanoyl chloride & $40292-82-8$ & 190.72 & 3.35 & $6.77 \mathrm{E}+01$ & Daphnia magna & 48 & & 47.00 & 1 \\
\hline Toluene, 4-chloro- & $106-43-4$ & 126.59 & 3.33 & $4.00 \mathrm{E}+01$ & Ceriodaphnia dubia & 48 & & 1.70 & 1 \\
\hline Benzene, 1-chloro-2-(chloromethyl)- & $611-19-8$ & 161.03 & 3.32 & $1.00 \mathrm{E}+02$ & Daphnia magna & 48 & 0.38 & & 1 \\
\hline Thymol & $89-83-8$ & & 3.30 & & & & & & 4 \\
\hline Phenol, 4-tert-butyl- & $98-54-4$ & 150.22 & 3.29 & $6.10 \mathrm{E}+02$ & $\begin{array}{l}\text { Daphnia magna } \\
\text { Daphnia magna }\end{array}$ & $\begin{array}{l}48 \\
48\end{array}$ & $\begin{array}{l}6.70 \\
3.40\end{array}$ & & 1 \\
\hline 2,6-di-tert-Butyl-4-ethylphenol & $4130-42-1$ & & 3.27 & $2.10 \mathrm{E}+01$ & & & & & 4 \\
\hline Cyclohexanamine, 4,4'-methylenebis- & $1761-71-3$ & 210.37 & 3.26 & $6.42 \mathrm{E}+02$ & Daphnia magna & 48 & & 6.84 & 1 \\
\hline Phenol, 2,4-dichloro- & $120-83-2$ & 163.00 & 3.25 & $4.50 \mathrm{E}+03$ & Daphnia magna & 48 & 1.40 & & 1 \\
\hline Silane, (3-chloropropyl)trichloro- & $2550-06-3$ & & 3.24 & $6.44 \mathrm{E}+01$ & & 列 & & & 4 \\
\hline Silane, tetramethyl- & $75-76-3$ & & 3.24 & $1.96 \mathrm{E}+01$ & Daphnia magna & 48 & & $>103$ & 3 \\
\hline Diallyl phthalate & $131-17-9$ & 246.26 & 3.23 & $1.48 \mathrm{E}+02$ & $\begin{array}{l}\text { Daphnia magna } \\
\text { Daphnia magna }\end{array}$ & $\begin{array}{l}48 \\
48\end{array}$ & $\begin{array}{c}5.50 \\
16.20\end{array}$ & & 1 \\
\hline Isononanol & 27458-94-2 & 144.26 & 3.22 & & & & & & 4 \\
\hline Diphenyl carbonate & $102-09-0$ & 214.22 & $\begin{array}{l}3.21- \\
3.28\end{array}$ & $1.30 \mathrm{E}+01$ & Daphnia magna & 48 & 6.50 & & 1 \\
\hline Pentane, 2-methyl- & $107-83-5$ & 86.18 & 3.21 & & & & & & 4 \\
\hline Hexamethylene diisocyanate & $822-06-0$ & 168.20 & 3.20 & & & & & & 4 \\
\hline 1,3-Xylene & $108-38-3$ & 106.17 & 3.20 & $1.61 \mathrm{E}+02$ & Daphnia magna & 48 & & 9.56 & 1 \\
\hline Peroxide, bis(tert-butyl)- & $110-05-4$ & & 3.20 & $1.71 \mathrm{E}+02$ & Daphnia magna & 48 & $>73.1$ & & 3 \\
\hline Benzene, 1,2-dichloro-3-nitro- & $3209-22-1$ & 192.00 & 3.20 & $7.41 \mathrm{E}+01$ & Daphnia magna & 24 & & 5.00 & 1 \\
\hline 1,4-Xylene & $106-42-3$ & 106.17 & 3.15 & $1.62 \mathrm{E}+02$ & Daphnia magna & 48 & & 8.50 & 1 \\
\hline Benzene, ethyl- & $100-41-4$ & 106.20 & 3.15 & $1.52 \mathrm{E}+02$ & $\begin{array}{l}\text { Crangon franciscorum } \\
\text { Daphnia magna }\end{array}$ & $\begin{array}{l}96 \\
48\end{array}$ & & $\begin{array}{c}2.00 \\
1.81-2.38\end{array}$ & 1 \\
\hline Ethanol, 2,2'-(dodecyloxidoimino)bis- & $2530-44-1$ & 289.46 & 3.13 & $2.99 \mathrm{E}+01$ & Daphnia magna & 48 & 10.80 & & 1 \\
\hline Silane, (3-chloropropyl)triethoxy- & $5089-70-3$ & & 3.13 & $1.13 \mathrm{E}+02$ & Daphnia magna & 48 & & 140.00 & 2 \\
\hline o-Xylene & $95-47-6$ & 106.17 & 3.12 & $1.78 \mathrm{E}+02$ & Daphnia magna & 48 & 3.20 & & 1 \\
\hline Stannane, dibutylbis[(1-oxododecyloxy]- & $77-58-7$ & & 3.12 & $7.00 \mathrm{E}-02$ & $\begin{array}{l}\text { Crangon franciscorum } \\
\text { Daphim mgong }\end{array}$ & 96 & 1.30 & 1938 & 2 \\
\hline \multirow{2}{*}{$\begin{array}{l}\text { Propane, 1-(ethenyloxy)-2-methyl- } \\
\text { Pall }\end{array}$} & $109-53-5$ & & 3.10 & $7.20 \mathrm{E}+02$ & $\begin{array}{l}\text { Dappnia magna } \\
\text { Daphnia magna }\end{array}$ & $\begin{array}{l}48 \\
48\end{array}$ & 46.30 & $1.9-3.8$ & $\begin{array}{l}2 \\
1\end{array}$ \\
\hline & & & & & Daphnia magna & 48 & & 0.29 & \\
\hline \multirow[t]{2}{*}{ Phenol, 4-chloro-2-methyl- } & $1570-64-5$ & 142.59 & 3.09 & $2.30 \mathrm{E}+03$ & Daphnia magna & 48 & & $\begin{array}{l}1.00 \\
0.63\end{array}$ & 1 \\
\hline & & & & & $\begin{array}{l}\text { Daphnia magna } \\
\text { Daphnia magna }\end{array}$ & $\begin{array}{l}48 \\
48\end{array}$ & & $\begin{array}{l}0.63 \\
>0.56\end{array}$ & \\
\hline Hexamethylene diacrylate & $13048-33-4$ & 226.27 & 3.08 & & & & & & 4 \\
\hline \multirow{2}{*}{ Dodecanedioic acid } & 693-23-2 & 230.30 & 3.07 & $3.00 \mathrm{E}+01$ & & & & & 4 \\
\hline & & & & & Daphnia magna & 24 & 3.00 & & \\
\hline \multirow{3}{*}{ 4-Nitrobenzene, 1,2-dichloro- } & 99-54-7 & 192.00 & 3.04 & $1.21 \mathrm{E}+02$ & Daphnia carinata & 48 & 8.20 & & 1 \\
\hline & & & & & Daphnia magna & 24 & 11.30 & & 1 \\
\hline & & & & & Daphnia & 48 & 3.00 & & \\
\hline Butyl methacrylate & $97-88-1$ & 142.20 & 3.03 & 3.60E+02 & $\begin{array}{l}\text { Daphnia magna } \\
\text { Daphnia magna }\end{array}$ & $\begin{array}{l}48 \\
48\end{array}$ & $\begin{array}{l}25.40 \\
32.00\end{array}$ & & 1 \\
\hline \multirow{2}{*}{ 1,3-Butadiene, 2,3-dichloro- } & $1653-19-6$ & 122.98 & 3.02 & & Daphnia magna & 48 & 1.5 & & 1 \\
\hline & $98-08-8$ & & 3.01 & $1.92 \mathrm{E}+02$ & $\begin{array}{l}\text { Daphnia magna } \\
\text { Daphnia magna }\end{array}$ & $\begin{array}{l}48 \\
48\end{array}$ & & 22.00 & 1 \\
\hline \multirow{3}{*}{$\begin{array}{l}\text { Dibutyltin, maleate- } \\
\text { 1-Octanol } \\
\text { Cyclopentane }\end{array}$} & $78-04-6$ & 346.99 & 3.01 & & $\begin{array}{l}\text { Daphnia magna } \\
\text { Daphnia magna }\end{array}$ & $\begin{array}{l}48 \\
48\end{array}$ & $\begin{array}{l}3.10 \\
0.21\end{array}$ & & 1 \\
\hline & $111-87-5$ & 130.23 & 3.00 & $5.51 \mathrm{E}+02$ & Daphnia magna & 24 & & 20.00 & 1 \\
\hline & $287-92-3$ & 70.14 & 3.00 & $1.56 \mathrm{E}+02$ & Daphnia magna & 48 & & 10.50 & 1 \\
\hline Citral & $5392-40-5$ & 152.23 & 3.00 & $5.90 \mathrm{E}+02$ & $\begin{array}{l}\text { Daphnia magna } \\
\text { Daphnia magna }\end{array}$ & $\begin{array}{l}24 \\
48\end{array}$ & & $\begin{array}{l}11.00 \\
7.00\end{array}$ & 1 \\
\hline \multirow{2}{*}{$\begin{array}{l}\text { Cyclohexene } \\
\text { 1,6-Octadien-3-ol, 3,7-dimethyl- }\end{array}$} & $110-83-8$ & 82.14 & 2.99 & $2.50 \mathrm{E}+02$ & Daphnia magna & 48 & 2.10 & & 1 \\
\hline & $78-70-6$ & 154.24 & 2.97 & 854-1589 & $\begin{array}{l}\text { Daphnia magna } \\
\text { Daphnia magna }\end{array}$ & $\begin{array}{l}48 \\
48\end{array}$ & & 59.00 & 1 \\
\hline \multirow{3}{*}{$\begin{array}{l}\text { 2-methylpropyl 2-methyl-2-propenoate } \\
\text { Styrene }\end{array}$} & & & & & $\begin{array}{l}\text { Daphnia magna } \\
\text { Daphnia magna }\end{array}$ & 48 & $>29$ & & 1 \\
\hline & $97-86-9$ & 142.20 & 2.95 & $4.70 \mathrm{E}+02$ & Daphnia magna & 48 & 23.00 & & 1 \\
\hline & $100-42-5$ & 104.15 & 2.95 & $3.00 \mathrm{E}+02$ & Daphnia magna & 48 & 4.70 & & 1 \\
\hline
\end{tabular}


(Table S6. continued)

\begin{tabular}{|c|c|c|c|c|c|c|c|c|c|}
\hline Chemicals name & CAS Reg. no & $\begin{array}{c}\text { Molecular } \\
\text { weight } \\
\left(\mathrm{g} \mathrm{mol}^{-1}\right)\end{array}$ & $\log K_{\text {ow }}$ & Solubility $\left(\mathrm{mg} \mathrm{L}^{-1}\right)$ & Species & $\begin{array}{l}\text { Duration } \\
\text { (h) }\end{array}$ & $\begin{array}{c}\text { Measured } \\
\mathrm{EC}_{50} \\
\left(\mathrm{mg} \mathrm{L}^{-1}\right) \\
\end{array}$ & $\begin{array}{c}\text { Nominal } \\
\mathrm{EC}_{50} \\
\left(\mathrm{mg} \mathrm{L}^{-1}\right) \\
\end{array}$ & Classification \\
\hline Benzene, 1,4-dichloro-2-nitro- & $89-61-2$ & & 2.93 & $9.50 \mathrm{E}+01$ & Daphnia magna & 24 & 8.00 & & 1 \\
\hline Benzene, (trichloromethyl)- & 98-07-7 & 195.48 & 2.92 & $1.00 \mathrm{E}+02$ & Daphnia magna & 24 & & $>100$ & 3 \\
\hline Dodecane-12-lactam & $947-04-6$ & 197.32 & 2.92 & $3.00 \mathrm{E}+02$ & Daphnia magna & 48 & 59.00 & & 1 \\
\hline Guanidine, N.N'-bis(2-methylphenyl)- & $97-39-2$ & & 2.90 & $7.00 \mathrm{E}+01$ & $\begin{array}{l}\text { Daphhia magna } \\
\text { Daphnia magna }\end{array}$ & $\begin{array}{l}24 \\
48\end{array}$ & $\begin{array}{l}41.00 \\
7.20\end{array}$ & & 1 \\
\hline Benzene, 2,4-dichloro-1-nitro- & $611-06-3$ & & 2.90 & $2.00 \mathrm{E}+02$ & Daphnia magna & & & $>0.56$ & 3 \\
\hline 1-Octanamine & $111-86-4$ & 129.25 & 2.90 & $1.00 \mathrm{E}+06$ & Daphnia magna & 48 & & 1.90 & 1 \\
\hline Phenol, 4-(1-methylethenyl)- & $4286-23-1$ & & 2.90 & $1.39 \mathrm{E}+03$ & Daphnia magna & 48 & 4.10 & & 1 \\
\hline \multirow{2}{*}{$\begin{array}{l}\text { 2-Propenoic acid, 2-ethyl-2-[[(1-oxo-2-propenyl)oxy]methyl]-1,3- } \\
\text { propanediyl }\end{array}$} & $15625-89-5$ & 296.32 & 2.86 & & & & & & 4 \\
\hline & & & & & Daphnia magna & 48 & 0.32 & & \\
\hline 2-Butene, 1,3-dichloro- & $926-57-8$ & 125.00 & 2.84 & 3.63E+02 & Daphnia magna & 48 & 11.00 & & 1 \\
\hline Methane, tetrachloro- & $56-23-5$ & 153.82 & 2.83 & $8.46 \mathrm{E}+02$ & $\begin{array}{l}\text { Dappnia manna } \\
\text { Daphnia magna }\end{array}$ & 48 & $\begin{array}{l}9.20 \\
8.10\end{array}$ & & 1 \\
\hline Dibutylamine & $111-92-2$ & 129.25 & 2.83 & & Daphnia magna & 48 & & 65.98 & 1 \\
\hline $\begin{array}{l}\text { Phosphoric acid, 2,2-bis(chloromethyl)-1,3-propanediyl tetrakis(2- } \\
\text { chloroethyl) }\end{array}$ & $38051-10-4$ & & 2.83 & $2.32 \mathrm{E}+02$ & Daphnia magna & 48 & & 42.00 & 1 \\
\hline 1-Hexanamine, 2-ethyl- & 104-75-6 & 129.24 & 2.82 & & & & & & 4 \\
\hline Butane, 1-chloro- & $109-69-3$ & & 2.82 & $3.70 \mathrm{E}+02$ & Daphnia Magna & 24 & & 380.00 & 1 \\
\hline Dicyclopentadiene & 77-73-6 & 132.20 & 2.78 & & Daphnia magna & 24 & 8.60 & & 1 \\
\hline 1-Hexanol, 2-ethyl- & $104-76-7$ & & 2.73 & & Daphnia magna & 48 & 8.00 & & 4 \\
\hline Butane, 2-methyl- & $78-78-4$ & & 2.72 & & Daphnia magna & 48 & 2.30 & & 1 \\
\hline \multirow{2}{*}{$\begin{array}{l}\text { Butane, 2-methyl- } \\
\text { Hexanal, 2-ethyl- }\end{array}$} & $123-05-7$ & 128.22 & 2.71 & $4.00 \mathrm{E}+02$ & Daphnia magna & 48 & & 11.50 & 1 \\
\hline & & & & & Daphnia magna & 48 & 0.23 & & \\
\hline \multirow{2}{*}{ Aniline, 3,4-dichloro- } & $95-76-1$ & 162.02 & 2.70 & $5.80 \mathrm{E}+02$ & Daphnia longispina & 48 & 0.44 & & 1 \\
\hline & & & & & Artemia salina & 48 & 9.00 & & \\
\hline 3H-Indol-3-one, 2-(1,3-dihydro-3-oxo-2H-indol-2-ylidene)-1,2-dihydro- & $482-89-3$ & & 2.70 & $9.90 \mathrm{E}+02$ & Daphnia magna & 24 & & 250.00 & 1 \\
\hline 2-methylpropyl 2-methyl-2-propanoate & $97-85-8$ & 144.21 & 2.68 & $5.20 \mathrm{E}+02$ & $\begin{array}{l}\text { Daphnia magna } \\
\text { Daphnia magna }\end{array}$ & $\begin{array}{l}48 \\
48\end{array}$ & 57.55 & & 1 \\
\hline \multirow{2}{*}{$\begin{array}{l}\text { 2-Butene, 2-methyl- } \\
\text { Benzyl chloride }\end{array}$} & 513-35-9 & 70.14 & 2.67 & $1.93 \mathrm{E}+02$ & $\begin{array}{l}\text { Daphnia magna } \\
\text { Daphnia magna }\end{array}$ & $\begin{array}{l}48 \\
48\end{array}$ & & 3.84 & 1 \\
\hline & $100-44-7$ & & 2.66 & $1.20 \mathrm{E}+03$ & Daphnia magna & 48 & 3.20 & & 1 \\
\hline Toluene & $108-88-3$ & 92.14 & 2.65 & $5.15 \mathrm{E}+02$ & Daphnia magna & 48 & & 11.50 & 1 \\
\hline \multirow{2}{*}{$\begin{array}{l}\text { Hexanoic acid, 2-ethyl- } \\
\text { Diisobutylamine }\end{array}$} & $149-57-5$ & 144.22 & 2.64 & & & & & & 4 \\
\hline & $110-96-3$ & 129.25 & 2.63 & & Daphnia magna & 48 & $>71$ & 3500 & 1 \\
\hline \multirow{5}{*}{$\begin{array}{l}\text { Dehydrolinalool } \\
\text { Silane, dichloroethenylmethyl- } \\
\text { Diisobutyl ketone } \\
2,2 \text { '-dimethyl-4,4'-methylenebis(cyclohexylamine) } \\
\text { Silane, dichloro(chloromethyl)methyl- }\end{array}$} & 29171-20-8 & 152.24 & 2.61 & $2.45 \mathrm{E}+03$ & $\begin{array}{l}\text { Daphnia magna } \\
\text { Danna }\end{array}$ & 48 & & 45.10 & 1 \\
\hline & $124-70-9$ & & 2.60 & $8.02 \mathrm{E}+02$ & & & & & 4 \\
\hline & $108-83-8$ & & 2.56 & $4.30 \mathrm{E}+02$ & Daphnia magna & 48 & & 250.00 & 1 \\
\hline & $6864-37-5$ & & 2.51 & $3.60 \mathrm{E}+03$ & Daphnia magna & 48 & & 15.20 & 1 \\
\hline & $1558-33-4$ & & 2.50 & $4.85 \mathrm{E}+02$ & & & & & 4 \\
\hline Silane, trichloroethyl- & $115-21-9$ & 163.51 & 2.50 & $9.87 \mathrm{E}+02$ & Daphnia magna & $\begin{array}{l}48 \\
48\end{array}$ & & 124.00 & 1 \\
\hline \multirow{3}{*}{$\begin{array}{l}\text { Silane, chlorotrimethyl- } \\
\text { Ethane, } 1,1,1 \text {-trichloro- } \\
\text { Benzoic acid, 4-methyl- }\end{array}$} & $75-77-4$ & & 2.48 & $1.01 \mathrm{E}+03$ & $\begin{array}{l}\text { Daphnia magna } \\
\text { Daphnia magna }\end{array}$ & $\begin{array}{l}48 \\
48\end{array}$ & 124.00 & & 1 \\
\hline & $71-55-6$ & & 2.47 & 3.00E +02 & Daphnia magna & 48 & 60.00 & & 1 \\
\hline & $99-94-5$ & & 2.44 & $3.49 \mathrm{E}+02$ & Daphnia magna & 48 & 42.00 & & 1 \\
\hline Ethene, trichloro- & $79-01-6$ & 131.39 & 2.42 & $1.10 \mathrm{E}+03$ & Daphnia cucullata & 48 & & $56-58$ & 1 \\
\hline \multirow{7}{*}{$\begin{array}{l}\text { Isoprene } \\
\text { 1-Butene } \\
\text { 1-Butene, 2,3,4-trichloro- } \\
\text { 2-Ethylhexyl mercaptoacetate } \\
\text { Hexanoyl chloride, 2-ethyl- } \\
\text { Hept-5-en-2-one, 6-methyl- } \\
\text { Silane, trichloroethenyl- }\end{array}$} & $78-79-5$ & 68.12 & 2.42 & $6.42 \mathrm{E}+02$ & Daphnia magna & $\begin{array}{l}48 \\
48\end{array}$ & 5.77 & 7.80 & 1 \\
\hline & $106-98-9$ & & 2.40 & & & & & & 4 \\
\hline & 2431-50-7 & 159.44 & 2.40 & $6.00 \mathrm{E}+02$ & Daphnia magna & 48 & & 50.00 & 1 \\
\hline & 7659-86-1 & & 2.40 & 4.73E+00 & Daphnia magna & 48 & 0.38 & & 2 \\
\hline & $760-67-8$ & 162.66 & 2.40 & $5.93 \mathrm{E}+02$ & Daphnia magna & 48 & & 85.40 & 1 \\
\hline & $110-93-0$ & 126.20 & 2.40 & $3.02 \mathrm{E}+03$ & Daphnia magna & 48 & & 129.00 & 1 \\
\hline & $75-94-5$ & & 2.40 & $1.34 \mathrm{E}+03$ & & & & & 4 \\
\hline \multirow{2}{*}{ Benzene, 1-chloro-4-nitro- } & $100-00-5$ & 157.55 & 239 & $2,43 \mathrm{E}+02$ & Daphnia magna & $\begin{array}{l}48 \\
24\end{array}$ & $\begin{array}{l}2.70 \\
1500\end{array}$ & & 1 \\
\hline & $100-00-5$ & 157.53 & 2.39 & $2.43 \mathrm{E}+02$ & $\begin{array}{l}\text { Dapphia magna } \\
\text { Daphnia magna }\end{array}$ & $\begin{array}{l}24 \\
48\end{array}$ & 6.70 & & 1 \\
\hline
\end{tabular}


(Table S6. continued)

\begin{tabular}{|c|c|c|c|c|c|c|c|c|c|}
\hline Chemicals name & CAS Reg. no & $\begin{array}{c}\text { Molecular } \\
\text { weight } \\
\left(\mathrm{g} \mathrm{mol}^{-1}\right)\end{array}$ & $\log \mathrm{K}_{\mathrm{ow}}$ & Solubility $\left(\mathrm{mg} \mathrm{L}^{-1}\right)$ & Species & $\begin{array}{l}\text { Duration } \\
\text { (h) }\end{array}$ & $\begin{array}{c}\text { Measured } \\
\mathrm{EC}_{50} \\
\left(\mathrm{mg} \mathrm{L}^{-1}\right)\end{array}$ & $\begin{array}{c}\text { Nominal } \\
\mathrm{EC}_{50} \\
\left(\mathrm{mg} \mathrm{L}^{-1}\right)\end{array}$ & Classification \\
\hline Ethane, 1,1,2,2-tetrachloro- & $79-34-5$ & 167.85 & 2.39 & $2.87 \mathrm{E}+03$ & $\begin{array}{l}\text { Daphnia magna } \\
\text { Daphnia magna }\end{array}$ & $\begin{array}{l}48 \\
48\end{array}$ & 23.00 & 9.30 & 1 \\
\hline Butyl acrylate & $141-32-2$ & 128.17 & 2.38 & $2.00 \mathrm{E}+03$ & Daphnia magna & 48 & 8.20 & & 1 \\
\hline \multirow[t]{2}{*}{ Benzoic acid, 3-methyl- } & 99-04-7 & 136.15 & 2.37 & $1.00 \mathrm{E}+03$ & Daphnia magna & 24 & & 75.00 & 1 \\
\hline & & & & & Daphnia magna & 48 & 11.80 & & \\
\hline Toluene, 4-nitro- & $99-99-0$ & 137.14 & 2.37 & $3.45 \mathrm{E}+02$ & $\begin{array}{l}\text { Daphnia magna } \\
\text { Daphnia manga }\end{array}$ & $\begin{array}{l}48 \\
48\end{array}$ & $\begin{array}{r}7.50 \\
4.20\end{array}$ & & 1 \\
\hline 1-Butene, 3,4-dichloro- & $760-23-6$ & 125.00 & 2.37 & $1.10 \mathrm{E}+03$ & Daphnia magna & $\begin{array}{l}40 \\
48\end{array}$ & & 10.00 & 1 \\
\hline Phenol, 4,4'-sulfonylbis- & $80-09-1$ & & 2.36 & $7.70 \mathrm{E}+02$ & Daphnia magna & 48 & & 100.00 & 1 \\
\hline Phenol, 2,6-dimethyl- & $576-26-1$ & 122.17 & 2.36 & & & & & & 4 \\
\hline Propene, 2-methyl- & $115-11-7$ & 56.11 & 2.34 & $2.63 \mathrm{E}-01$ & & & & & 4 \\
\hline 2-Butene, (2E)- & $624-64-6$ & 56.11 & 2.33 & $2.65 \mathrm{E}+02$ & Daphnid & 48 & & 23.00 & 1 \\
\hline 2-Butene, (2Z)- & $590-18-1$ & 56.11 & 2.33 & $7.00 \mathrm{E}+02$ & & 40 & & 2.00 & 4 \\
\hline \multirow[t]{3}{*}{ tert-Butyl acrylate } & $1663-39-4$ & & 2.32 & $2.00 \mathrm{E}+03$ & Daphnia magna & 48 & 8.74 & 5700 & 1 \\
\hline & & & & & Daphnia magna & $\begin{array}{l}48 \\
48\end{array}$ & 12.30 & 37.00 & \\
\hline & & & & & Daphnia magna & 24 & 13.20 & & \\
\hline \multirow{3}{*}{ Toluene, 2-nitro- } & $88-72-2$ & 137.14 & 2.30 & $4.37 \mathrm{E}+02$ & Daphnia magna & 48 & 10.90 & & 1 \\
\hline & & & & & Daphnia magna & 24 & 16.00 & & \\
\hline & & & & & Daphnia magna & 24 & 10.01 & & \\
\hline Aniline, 4-(1-methylethyl)- & 99-88-7 & & 2.30 & $2.39 \mathrm{E}+03$ & Daphnia magna & 48 & 1.50 & & 1 \\
\hline Ethane, 1,1-dichloro-1-fluoro- & $1717-00-6$ & & 2.30 & $4.00 \mathrm{E}+03$ & Daphnia magna & 48 & & 31.20 & 1 \\
\hline 2-Propenamide & $79-06-1$ & 131.50 & 2.29 & $1.10 \mathrm{E}+03$ & $\begin{array}{l}\text { Daphnia magna } \\
\text { Paratanytarsus parthenogenetica }\end{array}$ & $\begin{array}{l}48 \\
48\end{array}$ & $\begin{array}{r}98.00 \\
230.00\end{array}$ & & 1 \\
\hline 1-Butanethiol & $109-79-5$ & & 2.28 & $5.95 \mathrm{E}+02$ & Paratanytarsus parthenogenetica & 48 & 230.00 & & 4 \\
\hline -Picoline 5-thyl & & & $2.27-$ & $120 \mathrm{~F}+04$ & Daphnia magna & 24 & & 83.80 & \\
\hline \multirow{3}{*}{ Butane, 1-isocyanato- } & 104-90-5 & 121.18 & 2.52 & $1.20 \mathrm{E}+04$ & Daphnia magna & 48 & & 39.60 & 1 \\
\hline & $111-36-4$ & 99.13 & 2.26 & 202900 & Daphnia magna & 24 & & 43.00 & 1 \\
\hline & & & & 202900 & Daphnia magna & 48 & & $>100$ & 1 \\
\hline \multirow{3}{*}{ Dimethyl terephthalate } & $120-61-6$ & 194.18 & 2.25 & $190 \mathrm{E}+01$ & Daphnia magna & 48 & & $>100$ & 3 \\
\hline & & & & & Daphnia magna & 48 & & $>30$ & 3 \\
\hline & & & & & Daphnia carinata & 48 & 21.30 & & \\
\hline \multirow{3}{*}{ Benzene, 1-chloro-2-nitro- } & $88-73-3$ & 157.55 & 224 & $4,41 E+02$ & Daphnia magna & 24 & 12.00 & & 1 \\
\hline & & & & & Daphnia magna & 24 & 23.90 & & 1 \\
\hline & & & & & Daphnia magna & 48 & 3.20 & & \\
\hline Silane, dichlorodimethyl- & $75-78-5$ & 129.06 & 2.24 & $1.75 \mathrm{E}+03$ & Daphnia magna & 48 & $>117$ & & 3 \\
\hline Isobutyl acryliate & $106-63-8$ & & 2.22 & $2.00 \mathrm{E}+03$ & Daphnia magna & 38 & & 9.70 & 1 \\
\hline 1,2-ethanediyl 2-methyl-2-propenoate & $97-90-5$ & & 2.21 & & & & & & 4 \\
\hline o-Cresol & $95-48-7$ & 108.14 & 2.20 & $2.60 \mathrm{E}+04$ & Daphnia magna & 48 & $9.2-23.5$ & & 1 \\
\hline Chloroprene & $126-99-8$ & & 2.20 & $2.56 \mathrm{E}+02-4.80 \mathrm{E}+02$ & Daphnia magna & 48 & & 348.00 & 1 \\
\hline 3,4-Dimethylaniline & $95-64-7$ & & 2.17 & & Daphnia magna & 48 & 1.09 & & 1 \\
\hline Aniline, 2,6-dimethyl- & $87-62-7$ & & 2.17 & & Daphnia magna & 48 & 20.00 & & 1 \\
\hline 2,3-Dimethylaniline & $87-59-2$ & & 2.17 & & Daphnia magna & 48 & 8.90 & & 1 \\
\hline 3,5-Dimethylaniline & $108-69-0$ & 121.18 & 2.17 & $4.60 \mathrm{E}+03$ & Daphnia magna & 48 & & 2.20 & 1 \\
\hline 2-Propenoic acid, 2-methyl, 2-propenyl ester & $0096-05-9$ & 121.10 & 2.15 & $2.20 \mathrm{E}+03$ & Daphnia magna & 48 & 2.40 & 2.20 & 1 \\
\hline Stannane, trichlorooctyl- & $3091-25-6$ & 338.29 & 2.14 & 3.30E-01 & Daphnia magna & 48 & $>0.33$ & & 3 \\
\hline 2-Propanethiol, 2-methyl- & $75-66-1$ & & 2.14 & $2.05 \mathrm{E}+03$ & Daphnia magna & 48 & 6.70 & & 1 \\
\hline \multirow{2}{*}{ Stannane, dibutyldichloro- } & $683-18-1$ & 303.84 & 2.13 & $3.20 \mathrm{E}-01$ & Daphnia magna & 48 & & 0.84 & 2 \\
\hline & & & & & Daphnia magna & 48 & & 1.40 & 2 \\
\hline Benzene & $71-43-2$ & 78.11 & 2.13 & $1.80 \mathrm{E}+03$ & Daphnia magna & 48 & & 10.00 & 1 \\
\hline \multirow{4}{*}{$\begin{array}{l}\text { 2-Butenedioic acid (2E)-, diethyl ester } \\
\text { 1-Propene, hexafluoro- } \\
\text { Phenol, 3-methyl-4-nitro- } \\
\text { 1-Propene, 3-chloro- }\end{array}$} & $623-91-6$ & & 2.12 & $3.10 \mathrm{E}+03$ & Daphnia magna & $\begin{array}{l}24 \\
24\end{array}$ & & $\begin{array}{l}18.00 \\
11.00\end{array}$ & 1 \\
\hline & $116-15-4$ & 150.02 & 2.12 & & & & & & 4 \\
\hline & $2581-34-2$ & & 2.12 & $1.30 \mathrm{E}+01$ & Daphnia magna & 24 & & 9.10 & 1 \\
\hline & $107-05-1$ & & 2.10 & $3.60 \mathrm{E}+03$ & Daphnia magna & 24 & & 250.00 & 1 \\
\hline 3-(Trimethoxysilyl)propyl methacrylate & $2530-85-0$ & & 2.10 & $8.26 \mathrm{E}+01$ & Daphnia magna & $\begin{array}{l}48 \\
48\end{array}$ & 2876 & $>100$ & 3 \\
\hline
\end{tabular}


(Table S6. continued)

\begin{tabular}{|c|c|c|c|c|c|c|c|c|c|}
\hline Chemicals name & CAS Reg. no & $\begin{array}{c}\text { Molecular } \\
\text { weight } \\
\left(\mathrm{g} \mathrm{mol}^{-1}\right) \\
\end{array}$ & $\log \mathrm{K}_{\text {ow }}$ & Solubility $\left(\mathrm{mg} \mathrm{L}^{-1}\right)$ & Species & $\begin{array}{l}\text { Duration } \\
\text { (h) }\end{array}$ & $\begin{array}{c}\text { Measured } \\
E_{50} \\
\left(\mathrm{mg} \mathrm{L}^{-1}\right) \\
\end{array}$ & $\begin{array}{c}\text { Nominal } \\
E_{50} \\
\left(\mathrm{mg} \mathrm{L}^{-1}\right)\end{array}$ & Classification \\
\hline \multirow{3}{*}{ Stannane, tributylchloro- } & \multirow{3}{*}{$1461-22-9$} & & \multirow{3}{*}{2.07} & \multirow{3}{*}{$6.00 \mathrm{E}+00-1.00 \mathrm{E}+01$} & Daphnia magna & 48 & 0.02 & & \multirow{3}{*}{1} \\
\hline & & & & & Daphnia magna & 48 & & 0.01 & \\
\hline & & & & & $\begin{array}{l}\text { Daphnia magna } \\
\text { Daphnia magna }\end{array}$ & $\begin{array}{l}24 \\
24\end{array}$ & & $\begin{array}{l}0.01 \\
0.00\end{array}$ & \\
\hline \multirow{2}{*}{ Acrylonitrile } & \multirow{3}{*}{$\begin{array}{c}107-13-1 \\
17980-47-1\end{array}$} & \multirow{2}{*}{53.06} & 205 & \multirow{2}{*}{$7.35 \mathrm{E}+04$} & Daphnia magna & 48 & & 10.00 & \multirow[b]{2}{*}{1} \\
\hline & & & 2.05 & & Daphnia magna & 48 & & 7.60 & \\
\hline \multirow{4}{*}{$\begin{array}{l}\text { Silane, triethoxy(2-methylpropyl)- } \\
\text { 1-Hexanol } \\
\text { Silanamine, trimethyl-N-trimethylsilyl- } \\
\text { Cyclohexylamine, N,N-dimethyl- }\end{array}$} & & \multirow{4}{*}{$\begin{array}{l}102.18 \\
161.40\end{array}$} & 2.03 & \multirow{2}{*}{$\begin{array}{l}8.60 \mathrm{E}+01 \\
5.90 \mathrm{E}+03\end{array}$} & Daphnia magna & 48 & & $>49.1$ & \multirow{3}{*}{$\begin{array}{l}3 \\
1 \\
1\end{array}$} \\
\hline & $111-27-3$ & & 2.03 & & Daphnia magna & 24 & & \multirow{2}{*}{200.00} & \\
\hline & $999-97-3$ & & 2.02 & \multirow{4}{*}{$\begin{array}{l}7.61 \mathrm{E}+02 \\
1.34 \mathrm{E}+04\end{array}$} & Daphnia magna & 48 & 80.00 & & \\
\hline & $98-94-2$ & & 2.01 & & Daphnia magna & 48 & 75.00 & & 1 \\
\hline Silane, trichloromethyl- & \multicolumn{2}{|l|}{$75-79-6$} & 2.01 & & Daphnia magna & $\begin{array}{l}48 \\
48\end{array}$ & 124.00 & & 1 \\
\hline 2-Naphthol & $135-19-3$ & & $2.01-$ & & Daphnia magna & 48 & $>117$ & & \\
\hline 2-1vapitior & $103-12=2$ & & 2.84 & $600-900$ & Daphnia magna & 48 & & 3.54 & 1 \\
\hline $\begin{array}{l}\text { Propane, 1,2-dichloro- } \\
\text { 1,3-Butadiene }\end{array}$ & $\begin{array}{c}78-87-5 \\
106-99-0\end{array}$ & $\begin{array}{c}112.99 \\
54.09\end{array}$ & $\begin{array}{l}2.00 \\
1.99\end{array}$ & $\begin{array}{l}2.80 \mathrm{E}+03 \\
735 \mathrm{E}+02\end{array}$ & Daphnia magna & 48 & 55.90 & & 1 \\
\hline Promance & & & $1.98-$ & $1.35 \mathrm{E}+02$ & Daphnia magna & 48 & 20.00 & & 4 \\
\hline Propane, 1,2,3-trichloro- & $96-18-4$ & & 2.54 & $1.75 \mathrm{E}+03$ & Ceriodaphnia cf. dubia & 48 & 4.10 & & 1 \\
\hline 2,4-Dinitrotoluene & $121-14-2$ & 182.14 & 1.98 & & Daphnia magna & 24 & 31.20 & & 1 \\
\hline Ethane, 1,2-dibromo- & $106-93-4$ & & 1.96 & $3.91 \mathrm{E}+03$ & $\begin{array}{l}\text { Daphnia magna } \\
\text { Daphnia magna }\end{array}$ & 48 & $\begin{array}{l}30.40 \\
6.50\end{array}$ & & 1 \\
\hline & & & & & Daphnia magna & 24 & & 25.00 & \\
\hline Phenol, 3-methyl- & $108-39-4$ & 108.14 & 1.96 & $2.27 \mathrm{E}+04$ & Daphnia magna & 24 & & 8.90 & 1 \\
\hline & & & & & Daphnia pulicaria & 48 & $>99.5(\mathrm{e})$ & & \\
\hline 1,2,4-Benzenetricarboxylic acid, cyclic-1,2-anhydride & $552-30-7$ & & 1.95 & 2.10E+04 & Daphnia magna & 48 & $\begin{array}{c}\text { not } \\
\text { determined }\end{array}$ & & 3 \\
\hline & & & & & Daphnid & 48 & & 145.00 & \\
\hline & & & & & Mysid shrimp & 96 & & 44.00 & \\
\hline Ethane, 2-chloro-1,1,1,2-tetrafluoro- & $2837-89-0$ & 136.50 & 1.94 & $1.43 \mathrm{E}+03$ & Daphnia magna & 48 & & 31.20 & 1 \\
\hline & & & & & Daphnia magna & 48 & & 160.00 & \\
\hline Phenol, 4-methyl- & $106-44-5$ & 108.14 & 1.94 & $2.15 \mathrm{E}+04$ & $\begin{array}{l}\text { Daphnia magna } \\
\text { Daphnia pulicaria }\end{array}$ & 48 & & 4.90 & 1 \\
\hline & & & & & $\begin{array}{l}\text { Daphhia pulicaria } \\
\text { Daphnia magna }\end{array}$ & $\begin{array}{l}48 \\
48\end{array}$ & 22.70 & & \\
\hline Methane, bromo- & 74-83-9 & & 1.94 & $1.61 \mathrm{E}+04$ & $\begin{array}{l}\text { Daphina magna } \\
\text { Daphnia magna }\end{array}$ & $\begin{array}{l}48 \\
48\end{array}$ & & 2.60 & 1 \\
\hline Silane, chlorodimethyl- & $1066-35-9$ & & 1.93 & & & & & & 4 \\
\hline Ethane, 1,1,2-trichloro- & $79-00-5$ & & 1.89 & $2.87 \mathrm{E}+03$ & Daphnia magna & 48 & & 23.00 & 1 \\
\hline 2-Propenoic acid, 2-methyl-,1,2-ethanediylbis(oxy-2,1-ethanediyl)ester & 109-16-0 & 286.33 & 1.88 & & & & & & 4 \\
\hline Benzoic acid & $65-85-0$ & & 1.88 & $2.90 \mathrm{E}+03$ & $\begin{array}{l}\text { Daphnia magna } \\
\text { Daphnia magna }\end{array}$ & $\begin{array}{l}24 \\
48\end{array}$ & & 500.00 & 1 \\
\hline Fthyl methacrylate & $97-63-2$ & & & 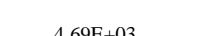 & Daphnia magna & 48 & $>66$ & & \\
\hline Etrnyı metnacrylate & 9l-63-2 & & 1.81 & 4.09E+03 & Daphnia magna & 48 & $>100$ & & 3 \\
\hline & & & & & Daphnia magna & 48 & 14.00 & & \\
\hline Aniline, 2-nitro- & $88-74-4$ & & 1.85 & $1.17 \mathrm{E}+03$ & Daphnia magna & 24 & 8.30 & & 1 \\
\hline & & & & & Daphnia magna & 48 & 4.90 & & 1 \\
\hline 2-Butene & $107-01-7$ & 56.10 & 1.85 & & Daphnia carinata & 48 & 10.50 & & \\
\hline Methacrylate, methyl- & $80-62-6$ & & 1.83 & $1.60 \mathrm{E}+04$ & Daphnia magna & & & & 4 \\
\hline 2,5-Dimethylaniline & $95-78-3$ & & 1.83 & & $\begin{array}{l}\text { Daphnia magnaa } \\
\text { Daphnia magna }\end{array}$ & $\begin{array}{l}48 \\
48\end{array}$ & $\begin{array}{l}09.00 \\
18.00\end{array}$ & & 1 \\
\hline p-Phenylenediamine, N-phenyl- & $101-54-2$ & & 1.82 & $5.04 \mathrm{E}+02$ & Daphnia magna & 48 & & 0.37 & 1 \\
\hline Tripropylene glycol diacrylate & $42978-66-5$ & 300.35 & 1.82 & & & & & & 4 \\
\hline Butyl acetate & $123-86-4$ & & 1.82 & $1.40 \mathrm{E}+04$ & & & & & 4 \\
\hline 1-Propanethiol & $107-03-9$ & & 1.81 & $1.90 \mathrm{E}+03$ & Daphnia magna & 48 & 70.00 & & 1 \\
\hline Thiophene & $110-02-1$ & & 1.81 & $3.02 \mathrm{E}+03$ & Daphnia magna & 48 & 21.00 & & 1 \\
\hline 2-Methylpropyl acetate & $110-19-0$ & & 1.78 & $6.00 \mathrm{E}+03$ & & & & & 4 \\
\hline 1-Propene & $115-07-1$ & & 1.77 & $2.00 \mathrm{E}+02$ & & & & & 4 \\
\hline Isophthalic acid & $121-91-5$ & & 1.76 & $1.30 \mathrm{E}+02$ & Daphnia magna & 48 & & - & 3 \\
\hline Benzaldehyde, 4-methoxy- & $123-11-5$ & & 1.76 & $4.40 \mathrm{E}+03$ & $\begin{array}{l}\text { Daphnia magna } \\
\text { Daphnia magna }\end{array}$ & $\begin{array}{l}48 \\
48\end{array}$ & 45.00 & 83.00 & 1 \\
\hline Ethane, 1,1,1-trifluoro- & $420-46-2$ & & 1.74 & $7.61 \mathrm{E}+02$ & Daphnia magna & 48 & & 300.00 & 1 \\
\hline Disulfide, bis(dimethylthiocarbamoyl)- & $137-26-8$ & & 1.73 & $1.80 \mathrm{E}+01$ & Daphnia magna & 48 & & 0.04 & 1 \\
\hline
\end{tabular}


(Table S6. continued)

\begin{tabular}{|c|c|c|c|c|c|c|c|c|c|}
\hline Chemicals name & CAS Reg. no & $\begin{array}{c}\text { Molecular } \\
\text { weight } \\
\left(\mathrm{g} \mathrm{mol}^{1}\right)\end{array}$ & $\log \mathrm{K}_{\mathrm{ow}}$ & Solubility $\left(\mathrm{mg} \mathrm{L}^{-1}\right)$ & Species & $\begin{array}{l}\text { Duration } \\
\text { (h) }\end{array}$ & $\begin{array}{c}\text { Measured } \\
\mathrm{EC}_{50} \\
\left(\mathrm{mg} \mathrm{L}^{-1}\right)\end{array}$ & $\begin{array}{c}\text { Nominal } \\
\mathrm{EC}_{50} \\
\left(\mathrm{mg} \mathrm{L}^{-1}\right)\end{array}$ & Classification \\
\hline $\begin{array}{l}\text { Cyanuric chloride } \\
\text { Silane, dichloromethyl- }\end{array}$ & $\begin{array}{l}108-77-0 \\
75-54-7\end{array}$ & 184.41 & $\begin{array}{l}1.70 \\
1.70\end{array}$ & $4.40 \mathrm{E}+02$ & & & & & $\begin{array}{l}4 \\
4\end{array}$ \\
\hline Guanidine, 1,3-diphenyl- & $102-06-7$ & & 1.69 & $4.75 \mathrm{E}+02$ & Daphnia magna & $\begin{array}{l}48 \\
24\end{array}$ & & $\begin{array}{l}17.00 \\
73.60\end{array}$ & 1 \\
\hline Methane, dibromo- & $74-95-3$ & & 1.68 & $9.00 \mathrm{E}+03$ & Daphnia magna & 48 & & 66.00 & 1 \\
\hline Butanoic acid, 2-ethyl & $88-09-5$ & & 1.68 & $1.70 \mathrm{E}+04$ & Daphnia magna & 48 & 70.00 & & 1 \\
\hline 2,4-Xylidine & $95-68-1$ & & 1.68 & & Daphnia magna & 48 & 9.90 & & 1 \\
\hline 2-Pentanol, 4-methyl- & $108-11-2$ & 102.17 & 1.68 & $1.64 \mathrm{E}+04$ & Daphnia magna & 48 & 337.00 & & 1 \\
\hline 2-Cyclohexen-1-one, 3,5,5-trimethyl- & $78-59-1$ & & 1.67 & $1.45 \mathrm{E}+04$ & Daphnia magna & 48 & 120.00 & & 1 \\
\hline Dipropylamine & $142-84-7$ & 101.19 & 1.67 & & & & & & 4 \\
\hline Phenol, 2,4-dinitro- & $51-28-5$ & & 1.67 & & & & & & 4 \\
\hline Benzaldehyde, 2-hydroxy- & $90-02-8$ & & 1.66 & $4.90 \mathrm{E}+03$ & Daphnia magna & 48 & 2.60 & & 1 \\
\hline Ethene, chlorotrifluoro- & $79-38-9$ & & 1.65 & $8.04 \mathrm{E}+02$ & & & & & 4 \\
\hline Ethane, 1-chloro-1,1-difluoro- & $75-68-3$ & 100.50 & $\begin{array}{l}1.64- \\
2.05\end{array}$ & $1.90 \mathrm{E}+03$ & Daphnia magna & 48 & & 160.00 & 1 \\
\hline Hydroperoxide, 1-methyl-1-phenylethyl- & $80-15-9$ & & 1.60 & $1.43 \mathrm{E}+05$ & Daphnia magna & 48 & 18.00 & & 1 \\
\hline 1,3-Isobenzofurandione & $85-44-9$ & & 1.60 & & Daphnia magna & 24 & & 140.00 & 1 \\
\hline Ethene, ethoxy- & $109-92-2$ & & 1.60 & $7.80 \mathrm{E}+03$ & Daphnia magna & 48 & & $>100$ & 3 \\
\hline Aniline, 4,4'-methylenebis- & $101-77-9$ & 198.30 & 1.59 & $1.25 \mathrm{E}+03$ & Moina macrocopa & 24 & & 2.30 & 1 \\
\hline Ethene, chloro- & $75-01-4$ & & 1.58 & $1.10 \mathrm{E}+03$ & & & & & 4 \\
\hline 2-Butoxyethyl acetate & $112-07-2$ & & 1.57 & $1.50 \mathrm{E}+04$ & $\begin{array}{l}\text { Daphnia magna } \\
\text { Daphnia magna }\end{array}$ & $\begin{array}{l}48 \\
24\end{array}$ & 143.00 & 150.00 & 1 \\
\hline tert-Amyl methyl ether & $994-05-8$ & & 1.55 & $1.10 \mathrm{E}+04$ & $\begin{array}{l}\text { Dappnia magna } \\
\text { Daphnia magna }\end{array}$ & 48 & 100.00 & & 1 \\
\hline Ethanol, 2-(hexyloxy)- & $112-25-4$ & & 1.55 & $9.90 \mathrm{E}+03$ & Daphnia magna & 48 & & 145.00 & 1 \\
\hline Aniline, 3-nitro- & $99-09-2$ & 138.14 & 1.54 & $1.14 \mathrm{E}+03$ & $\begin{array}{l}\text { Daphnia magna } \\
\text { Daphnia magna }\end{array}$ & $\begin{array}{l}48 \\
24\end{array}$ & 305.00 & 3600 & 1 \\
\hline Pyridine, 2-vinyl- & $100-69-6$ & & 1.54 & $2.67 \mathrm{E}+04$ & Daphnia magna & $\begin{array}{l}24 \\
48\end{array}$ & 9.50 & 36.00 & 1 \\
\hline Carbonochloridic acid, 1-methylpropyl ester & $17462-58-7$ & 136.58 & 1.54 & $4.22 \mathrm{E}+03$ & & & & & 4 \\
\hline 1-Propanol, butoxy- & $29387-86-8$ & & 1.52 & $5.50 \mathrm{E}+04$ & & & & & 4 \\
\hline 2-Propanol, 1-phenoxy- & $770-35-4$ & 152.19 & 1.52 & $1.00 \mathrm{E}+04$ & Daphnia magna & 48 & & $<100$ & 3 \\
\hline Propanenitrile, 3-(trichlorosilyl)- & $1071-22-3$ & & 1.52 & $1.37 \mathrm{E}+03$ & & & & & 4 \\
\hline 1,3-Pentadiene & $504-60-9$ & 68.00 & 1.50 & $6.90 \mathrm{E}+02$ & Daphnia magna & 48 & & 221.50 & 1 \\
\hline Propylene glycol phenyl ether (beta isomer - primary alcohol) & 4169-04-4 & & 1.50 & $1.00 \mathrm{E}+04$ & & & & & 4 \\
\hline 1,4-Cyclohexanedimethanol & $105-08-8$ & & 1.49 & $9.20 \mathrm{E}+05$ & Daphnia magna & 48 & & $>100$ & 3 \\
\hline Benzaldehyde & $100-52-7$ & 106.12 & 1.48 & $6.55 \mathrm{E}+03$ & Daphnia magna & 24 & & 50.00 & 1 \\
\hline Ethane, pentafluoro- & $354-33-6$ & & 1.48 & $4.32 \mathrm{E}-01-1.07 \mathrm{E}+00$ & & & & & 4 \\
\hline Phenol & $108-95-2$ & & 1.47 & $8.40 \mathrm{E}+04$ & $\begin{array}{l}\text { Daphnia magna } \\
\text { Daphnia magna }\end{array}$ & $\begin{array}{l}24 \\
48\end{array}$ & & $\begin{array}{l}12.00 \\
12.00\end{array}$ & 1 \\
\hline Silane, trichloro- & $10025-78-2$ & & 1.46 & & & & & & 4 \\
\hline $\begin{array}{l}\text { Triethylamine } \\
\text { S }\end{array}$ & $121-44-8$ & 101.19 & 1.45 & & Daphnia magna & 48 & & 200.00 & 1 \\
\hline Ethane, 1,2-dichloro- & $107-06-2$ & & 1.45 & $8.49 \mathrm{E}+03-9.00 \mathrm{E}+03$ & Daphnia magna & 24 & 150.00 & & 1 \\
\hline \multirow[t]{4}{*}{ Ethane, chloro- } & $75-00-3$ & & 1.43 & $5.74 \mathrm{E}+03$ & Daphnia magna & 48 & 150.00 & 58.00 & 1 \\
\hline & & & & & Daphnia magna & 48 & 15.60 & & \\
\hline & & & & & Daphnia magna & 48 & 5.00 & & \\
\hline & & & & & Daphnia magna & 48 & 0.52 & & \\
\hline \multirow[t]{4}{*}{ Aniline, 2-methyl- } & $95-53-4$ & & 1.40 & $1.50 \mathrm{E}+04$ & Daphnia magna & 72 & 0.62 & & 1 \\
\hline & & & & & Daphnia magna & 24 & 6.60 & & \\
\hline & & & & & Daphnia magna & 24 & 26.00 & & \\
\hline & & & & & Daphnia magna & 48 & 4.80 & & \\
\hline 2-Propanamine, N-(1-methylethyl)- & $108-18-9$ & 101.19 & 1.40 & & Daphnia magna & 48 & & 110.00 & 1 \\
\hline m-Toluidine & $108-44-1$ & 107.16 & 1.40 & $1.00 \mathrm{E}+04$ & Daphnia magna & 24 & & 3.80 & 1 \\
\hline Hydroperoxide, 1,1-dimethylpropyl- & $3425-61-4$ & & 1.40 & & & & & & 4 \\
\hline Butyric anhydride & $106-31-0$ & & 1.39 & $4.56 \mathrm{E}+03$ & $\begin{array}{l}\text { Daphnia magna } \\
\text { Daphnia magna Straus }\end{array}$ & $\begin{array}{l}48 \\
48\end{array}$ & & $\begin{array}{l}22.70 \\
51.25\end{array}$ & 1 \\
\hline Aniline, 4-methyl- & $106-49-0$ & & 1.39 & $7.40 \mathrm{E}+03$ & Daphnia magna & 48 & 0.12 & & 1 \\
\hline Pentanoic acid & $109-52-4$ & 102.13 & 1.39 & $2.40 \mathrm{E}+04$ & $\begin{array}{l}\text { Daphnia magna } \\
\text { Daphnia magna }\end{array}$ & $\begin{array}{l}48 \\
48\end{array}$ & & $\begin{array}{l}88.10 \\
45.00\end{array}$ & 1 \\
\hline 1,3,5-Triazine-2,4-diamine, 6-phenyl- & $91-76-9$ & & 1.38 & $3.20 \mathrm{E}+02$ & $\begin{array}{l}\text { Daphnia magna } \\
\text { Daphnia magna }\end{array}$ & $\begin{array}{l}48 \\
48\end{array}$ & 52.00 & & 1 \\
\hline Pentanal & $110-62-3$ & & 1.38 & $1.17 \mathrm{E}+04$ & $\begin{array}{l}\text { Daphnia magna } \\
\text { Daphnia magna }\end{array}$ & $\begin{array}{l}48 \\
48\end{array}$ & $\begin{array}{l}70.70 \\
>100\end{array}$ & & 1 \\
\hline
\end{tabular}


(Table S6. continued)

\begin{tabular}{|c|c|c|c|c|c|c|c|c|c|}
\hline Chemicals name & CAS Reg. no & $\begin{array}{c}\text { Molecular } \\
\text { weight } \\
\left(\mathrm{g} \mathrm{mol}^{-1}\right)\end{array}$ & $\log \mathrm{K}_{\mathrm{ow}}$ & Solubility $\left(\mathrm{mg} \mathrm{L}^{-1}\right)$ & Species & $\begin{array}{l}\text { Duration } \\
\text { (h) }\end{array}$ & $\begin{array}{c}\text { Measured } \\
\mathrm{EC}_{50} \\
\left(\mathrm{mg} \mathrm{L}^{-1}\right)\end{array}$ & $\begin{array}{c}\text { Nominal } \\
E_{50} \\
\left(\mathrm{mg} \mathrm{L}^{-1}\right)\end{array}$ & Classification \\
\hline Benzoic acid, 4-hydroxy- & $99-96-7$ & & 1.37 & $6.00 \mathrm{E}+03$ & Daphnia Magna. & 48 & & 135.70 & 1 \\
\hline 3-Penten-2-one, 4-methyl- (Mesityl oxide) & $141-79-7$ & 98.15 & 1.37 & $3.00 \mathrm{E}+04$ & $\begin{array}{l}\text { Daphnia magna } \\
\text { Daphnia magna }\end{array}$ & $\begin{array}{l}48 \\
48\end{array}$ & & $\begin{array}{l}406.00 \\
970.00\end{array}$ & 1 \\
\hline \multirow{2}{*}{ Vanillin } & $121-33-5$ & 152.14 & 1.35 & $1.00 \mathrm{E}+04$ & Daphnia magna & 24 & & 180.00 & 1 \\
\hline & & & & & Daphnia magna & 24 & 8370.00 & & \\
\hline \multirow[t]{2}{*}{ Trichloroacetic acid } & $76-03-9$ & & 1.33 & $1.20 \mathrm{E}+04$ & $\begin{array}{l}\text { Daphnia magna } \\
\text { Daphnia magna }\end{array}$ & $\begin{array}{l}24 \\
48\end{array}$ & $>10000$ & & 1 \\
\hline & & & & & Daphnia magna & 24 & 110.00 & & \\
\hline Butanal, 3-methyl- & $590-86-3$ & & 1.32 & $2.00 \mathrm{E}+04$ & Daphnia magna & 48 & & 177.00 & 1 \\
\hline Ethanol, 2-(2-butoxyethoxy)-, acetate & $124-17-4$ & 204.27 & 1.30 & & Daphnia magna & 48 & & 143.00 & 1 \\
\hline 2H-Pyran, 3,4-dihydro-2-methoxy- & 4454-05-1 & 114.14 & 1.30 & $1.60 \mathrm{E}+04$ & $\begin{array}{l}\text { Daphnia magna } \\
\text { Daphnia magna }\end{array}$ & $\begin{array}{l}48 \\
48\end{array}$ & & $\begin{array}{l}>100 \\
176.78\end{array}$ & 1 \\
\hline Aniline, 4-ethoxy- & $156-43-4$ & 137.18 & 1.28 & $2.10 \mathrm{E}+04$ & Daphnia magna & 24 & & 170.00 & 1 \\
\hline Ethanethiol & $75-08-1$ & & 1.27 & $1.56 \mathrm{E}+04$ & $\begin{array}{l}\text { Daphnia magna } \\
\text { Daphnia magna }\end{array}$ & $\begin{array}{l}24 \\
48\end{array}$ & & $\begin{array}{l}<0.38 \\
90-280\end{array}$ & 1 \\
\hline Ethanol, 2-[2-(hexyloxy)ethoxy]- & $112-59-4$ & 190.29 & 1.27 & $1.70 \mathrm{E}+04$ & Daphnia magna & 48 & & 433.00 & 1 \\
\hline Methane, dichloro- & $75-09-2$ & & 1.25 & $1.32 \mathrm{E}+04$ & Daphnia magna & 48 & 27.00 & & 1 \\
\hline Propanoic acid, 2-methyl-, anhydride & $97-72-3$ & & 1.24 & $1.60 \mathrm{E}+04$ & $\begin{array}{l}\text { Palamomonetes pugio } \\
\text { Daphnia magna Straus }\end{array}$ & $\begin{array}{l}48 \\
48\end{array}$ & $\begin{array}{l}109.00 \\
51.25\end{array}$ & & 1 \\
\hline Propyl acetate & $109-60-4$ & & 1.24 & $2.00 \mathrm{E}+04$ & Daphnia magna & 48 & $>970$ & & 3 \\
\hline Ethene, 1,1-difluoro- & $75-38-7$ & 64.00 & 1.24 & $2.54 \mathrm{E}+02$ & & & & & 4 \\
\hline Propyl chloroformate & $109-61-5$ & 122.55 & 1.22 & $1.09 \mathrm{E}+04$ & & & & & 4 \\
\hline Ethyl acrylate & $140-88-5$ & & 1.18 & $1.50 \mathrm{E}+04$ & Daphnia magna & 48 & & 4.41 & 1 \\
\hline Aniline, 2-methoxy- & $90-04-0$ & & 1.18 & & & & & & 4 \\
\hline Butanoic acid, 2-methyl- & $116-53-0$ & 102.13 & 1.18 & & & & & & 4 \\
\hline Terephthalic acid & $100-21-0$ & & 1.16 & $1.90 \mathrm{E}+04$ & Daphnia magna & & $>982$ & & 3 \\
\hline Ethanol, 2-phenoxy- & $122-99-6$ & 138.17 & 1.16 & $2.89 \mathrm{E}+04$ & Daphnia magna & 48 & & $>500$ & 3 \\
\hline Acetamide, N-phenyl- & $103-84-4$ & & 1.16 & $4.00 \mathrm{E}+03$ & Daphnia magna & 48 & & $>100$ & 3 \\
\hline Methyl isobutyl ketone & $108-10-1$ & 100.16 & 1.16 & $1.70 \mathrm{E}+04-2.04 \mathrm{E}+04$ & Daphnia magna & 48 & & 4300.00 & 1 \\
\hline Phthalimide & $85-41-6$ & & 1.15 & $3.70 \mathrm{E}+02$ & Daphnia magna & 48 & 20.80 & & 1 \\
\hline 2-Propanol, 1-butoxy- & $5131-66-8$ & & 1.15 & $5.50 \mathrm{E}+04$ & & & & & 4 \\
\hline 2,5,7,10-Tetraoxa-6-silaundecane, 6-ethenyl-6-(2-methoxyethoxy)- & $1067-53-4$ & & 1.14 & $5.00 \mathrm{E}+03-1.00 \mathrm{E}+06$ & & & & & 4 \\
\hline 2-(Dimethylamino)ethyl methacrylate & $2867-47-2$ & & 1.13 & $1.06 \mathrm{E}+05$ & Daphnia magna & 48 & 33.00 & & 1 \\
\hline 2-Propanol, 1-(2-butoxy-1-methylethoxy)- & 29911-28-2 & 190.29 & 1.13 & $4.50 \mathrm{E}+04$ & & & & & 4 \\
\hline Ethene & 74-85-1 & & 1.13 & $1.31 \mathrm{E}+02$ & & & & & 4 \\
\hline Phosphoric acid, triethyl ester & $78-40-0$ & & 1.11 & miscible & Daphnia magna & 48 & & 350.00 & 1 \\
\hline \multirow{2}{*}{ Benzenemethanol } & $100-51-6$ & & 1.10 & $4.00 \mathrm{E}+04$ & Daphnia magna & 24 & & 400.00 & 1 \\
\hline & & & & & Daphnia magna & $\begin{array}{l}48 \\
48\end{array}$ & & 360.00 & \\
\hline \multirow{2}{*}{ Propanenitrile, 2,2'-azobis[2-methyl- } & $78-67-1$ & & 1.10 & $3.50 \mathrm{E}+02$ & $\begin{array}{l}\text { Daphnia Magna. } \\
\text { Daphnia Magna }\end{array}$ & $\begin{array}{l}48 \\
48\end{array}$ & & $>367$ & 3 \\
\hline & $108-23-6$ & 122.55 & 1.04 & $1.26 \mathrm{E}+04$ & & & & & 4 \\
\hline 1-methylethyl acetate & $108-21-4$ & & 1.02 & $3.09 \mathrm{E}+04$ & Daphnia magna & 24 & & 4150.00 & 1 \\
\hline Aniline, 3-methoxy- & 536-90-3 & 123.00 & 1.01 & $2.05 \mathrm{E}+04$ & Daphnia magna & 24 & & 100.00 & 1 \\
\hline Pyrocatechol & $120-80-9$ & & 1.01 & $4.49 \mathrm{E}+05$ & Daphnia magna & 24 & 1.70 & & 1 \\
\hline Cyclohexylamine, 3-aminomethyl-3,5,5-trimethyl- & 2855-13-2 & 170.30 & 0.99 & miscible & Daphnia magna & 48 & 23.00 & & 1 \\
\hline 1-Butanamine & $109-73-9$ & 73.14 & 0.97 & & Ceriodaphnia dubia & 48 & 8.20 & & 1 \\
\hline Diethyl malonate & $105-53-3$ & & 0.96 & $2.00 \mathrm{E}+04$ & Daphnia magna & 48 & & 202.00 & 1 \\
\hline Glycidyl methacrylate & $106-91-2$ & & 0.96 & $5.00 \mathrm{E}+04$ & Daphnia magna & 48 & 24.90 & & 1 \\
\hline \multirow[t]{2}{*}{ 1,2,4-Benzenetricarboxylic acid } & $528-44-9$ & & 0.95 & $2.10 \mathrm{E}+04$ & Daphnia magna & 48 & & $\begin{array}{l}\text { not } \\
\text { determined }\end{array}$ & 3 \\
\hline & & & & & Daphnia magna & 48 & 651.40 & & \\
\hline \multirow{3}{*}{ Methyl t-butyl ether } & 163204040 & & & & Daphnia magna & 48 & 472.00 & & 1 \\
\hline & $1634-04-4$ & 88.15 & 0.94 & & $\begin{array}{l}\text { Brachionus calyciflorus } \\
\text { Physa gyrina }\end{array}$ & $\begin{array}{l}24 \\
96\end{array}$ & $\begin{array}{l}960.00 \\
559.00\end{array}$ & & 1 \\
\hline & & & & & Hexagenia limbata & 96 & 581.00 & & \\
\hline Propanoic acid, 2-methyl- & $79-31-2$ & & 0.94 & $1.67 \mathrm{E}+02$ & Daphnia magna Straus & 48 & & 51.25 & 1 \\
\hline Propane, 2-nitro- & $79-46-9$ & & 0.93 & $1.70 \mathrm{E}+04$ & Daphnid & 24 & 290.00 & & 1 \\
\hline Methacrylic acid & $79-41-4$ & & 0.93 & $8.90 \mathrm{E}+04$ & Selenastrum capricornutum & 72 & & 45.00 & 1 \\
\hline Dimethyl sulfide & $75-18-3$ & & 0.92 & $2.00 \mathrm{E}+04$ & $\begin{array}{l}\text { Daphnia magna } \\
\text { Daphnia magna }\end{array}$ & $\begin{array}{l}48 \\
48\end{array}$ & & $\begin{array}{l}81.00 \\
29.00\end{array}$ & 1 \\
\hline
\end{tabular}


(Table S6. continued)

\begin{tabular}{|c|c|c|c|c|c|c|c|c|c|}
\hline Chemicals name & CAS Reg. no & $\begin{array}{c}\text { Molecular } \\
\text { weight } \\
\left(\mathrm{g} \mathrm{mol}^{-1}\right)\end{array}$ & $\log \mathrm{K}_{\mathrm{ow}}$ & Solubility $\left(\mathrm{mg} \mathrm{L}^{-1}\right)$ & Species & $\begin{array}{l}\text { Duration } \\
\text { (h) }\end{array}$ & $\begin{array}{c}\text { Measured } \\
\mathrm{EC}_{50} \\
\left(\mathrm{mg} \mathrm{L}^{-1}\right)\end{array}$ & $\begin{array}{c}\text { Nominal } \\
\mathrm{EC}_{50} \\
\left(\mathrm{mg} \mathrm{L}^{-1}\right)\end{array}$ & Classification \\
\hline 2-Buten-1-ol, 3-methyl- & $556-82-1$ & & 0.91 & $1.70 \mathrm{E}+05$ & Daphnia magna & 48 & & 144.00 & 1 \\
\hline Methane, chloro- & $74-87-3$ & 50.49 & 0.91 & $4.80 \mathrm{E}+03-5.32 \mathrm{E}+03$ & Daphnia magna & 48 & & 200.00 & 1 \\
\hline \multirow[t]{2}{*}{ Propanoic acid, 3-mercapto-, methyl ester } & 2935-90-2 & & 0.90 & $2.10 \mathrm{E}+04$ & Daphnia magna & 48 & 0.55 & & 1 \\
\hline & & & & & Daphnia pulex & 48 & & 0.10 & \\
\hline \multirow{3}{*}{ Aniline } & $62-53-3$ & & 0.90 & $3.50 \mathrm{E}+04$ & Daphnia magna & 48 & & 0.17 & 1 \\
\hline & & & & & Daphnia magna & 48 & & 0.30 & \\
\hline & & & & & Daphnia magna & 24 & & 85.00 & \\
\hline \multirow[t]{2}{*}{ Phenol, 2,4,6-trinitro- } & $88-89-1$ & & 0.89 & $1.18 \mathrm{E}+04$ & Daphnia magna & 48 & & 90.00 & 1 \\
\hline & & & & & Daphnia magna & 48 & & 86.00 & \\
\hline Propanoyl chloride, 2,2-dimethyl- & $3282-30-2$ & 120.58 & 0.89 & $3.48 \mathrm{E}+04$ & Daphnia magna & 48 & & 202.94 & 1 \\
\hline $\mathrm{n}$-Butanal & $123-72-8$ & 72.11 & 0.88 & $1.18 \mathrm{E}+05$ & Daphnia magna & 24 & & 194.00 & 1 \\
\hline \multirow[t]{2}{*}{ 1-Butanol } & $71-36-3$ & & 0.88 & $7.70 \mathrm{E}+04$ & $\begin{array}{l}\text { Daphnia magna } \\
\text { Daphnia magna }\end{array}$ & $\begin{array}{l}48 \\
48\end{array}$ & 1328.00 & 2337.00 & 1 \\
\hline & & & & & Daphnia magna & 48 & 931.00 & & \\
\hline \multirow[t]{2}{*}{ Butanamide, N-(2-methylphenyl)-3-oxo- } & $93-68-5$ & & 0.85 & $3.00 \mathrm{E}+03$ & Daphnia magna & 96 & 41.10 & & 1 \\
\hline & & & & & Daphnia magna & 96 & $10-100$ & & \\
\hline o-Toluenesulfonamide & $88-19-7$ & & 0.84 & $1.60 \mathrm{E}+03$ & Daphnia magna & 48 & 210.00 & & 1 \\
\hline D-Mannitol & $69-65-8$ & 171.23 & 0.84 & & & & & & 4 \\
\hline Carbon dioxide & $124-38-9$ & 44.01 & 0.83 & & & & & & 4 \\
\hline $\mathrm{p}$-Toluenesulfonamide & $70-55-3$ & 7.0.01 & 0.82 & $3.20 \mathrm{E}+03$ & Daphnia magna & 24 & & 150.00 & 1 \\
\hline Ethanol, 2-butoxy- & $111-76-2$ & & 0.80 & $5.00 \mathrm{E}+01$ & & & & & 4 \\
\hline 1,3-Benzenediol & $108-46-3$ & & 0.80 & $7.17 \mathrm{E}+05$ & Daphnia magna & 48 & & 1.28 & 1 \\
\hline Butanoic acid & $107-92-6$ & & 0.79 & miscible & Daphnia magna & 24 & & 1950.00 & 1 \\
\hline Propane, 1-nitro- & $108-03-2$ & & 0.79 & $1.40 \mathrm{E}+04$ & $\begin{array}{l}\text { Daphnia magna } \\
\text { Daphnia magna }\end{array}$ & $\begin{array}{l}48 \\
48\end{array}$ & & 380.00 & 1 \\
\hline & & & & & $\begin{array}{l}\text { Daphnia magna } \\
\text { Daphnia magna Straus }\end{array}$ & $\begin{array}{l}48 \\
48\end{array}$ & & $\begin{array}{l}258.00 \\
1300.00\end{array}$ & \\
\hline 1-Propanol, 2-methyl- & $78-83-1$ & 74.12 & 0.79 & $8.50 \mathrm{E}+04$ & Daphnia pulex & 48 & & 1100.00 & 1 \\
\hline Methanethiol & $74-93-1$ & & 0.78 & $2.33 \mathrm{E}+01$ & & & & & 4 \\
\hline Propanal, 2-methyl- & $78-84-2$ & 72.11 & 0.77 & $8.90 \mathrm{E}+04$ & $\begin{array}{l}\text { Daphnia magna Strauss } \\
\text { Daphnia magna Strauss }\end{array}$ & $\begin{array}{l}48 \\
48\end{array}$ & & $\begin{array}{l}308.00 \\
277.00\end{array}$ & 1 \\
\hline Hexane-1,6-diol & $629-11-8$ & 118.18 & 0.76 & miscible & Daphnia magna & 48 & & $>500$ & 3 \\
\hline Acetic acid, cyano- & $372-09-8$ & & 0.76 & $8.90 \mathrm{E}+05-1.00 \mathrm{E}+06$ & Daphnia magna & 48 & & 59.20 & 1 \\
\hline Ethyl cyanoacetate & $105-56-6$ & & 0.76 & $2.00 \mathrm{E}+04$ & Daphnia magna & 48 & & 471.00 & 1 \\
\hline \multirow{2}{*}{ Ethane, 1,1-difluoro- } & $75-37-6$ & 66.10 & 0.75 & $2.67 \mathrm{E}+03$ & Daphnia magna & 48 & & 980.00 & 1 \\
\hline & & & & & Daphnia magna & 48 & 2.60 & & \\
\hline \multirow[t]{2}{*}{ Methyl acrylate } & $96-33-3$ & & 0.74 & $5.20 \mathrm{E}+04$ & Mysidopsis bahia & 96 & 1.60 & & 1 \\
\hline & 年 & & . & Thent & Daphnia magna & 48 & 2.21 & & te \\
\hline Silanetriol, ethyl-, triacetate & $17689-77-9$ & 234.28 & 0.74 & 4.16E+04 & Daphnia magna & 48 & & 62.00 & 1 \\
\hline 2-Butanamine & $13952-84-6$ & 73.14 & 0.74 & 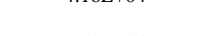 & Daphnia magna & 48 & & 40.00 & 1 \\
\hline Triethyl phosphite & $122-52-1$ & 166.16 & 0.74 & $1.50 \mathrm{E}+04$ & Daphnia magna & 24 & & 94.10 & 1 \\
\hline Vinyl acetate & $108-05-4$ & 86.09 & 0.73 & & & & & & 4 \\
\hline Ethyl acetate & $141-78-6$ & & 0.73 & $8.30 \mathrm{E}+04$ & Daphnia magna & 24 & & 3090.00 & 1 \\
\hline Ethylamine, $\mathrm{N}, \mathrm{N}$-dimethyl- & $598-56-1$ & 73.14 & 0.70 & & $\begin{array}{l}\text { Dappnia magna } \\
\text { Daphnia manga }\end{array}$ & $\begin{array}{l}24 \\
48\end{array}$ & & $\begin{array}{c}2500.00 \\
39.23\end{array}$ & 1 \\
\hline Acetoacetanilide & $102-01-2$ & & 070 & $8.375-10$ & Daphnia magna & 24 & & $70-200$ & 1 \\
\hline Acetoacetanninae & $102-01-2$ & & 0.10 & $8.3 / 5-10$ & Daphnia magna & 96 & & $>100$ & 1 \\
\hline Hydroperoxide, tert-butyl- & $\begin{array}{r}75-91-2 \\
87-56-9\end{array}$ & 90.12 & 0.70 & $2.20 \mathrm{E}+01$ & & & & & 4 \\
\hline Mucochloric acid & $87-56-9$ & & 0.70 & $2.70 \mathrm{E}+04$ & Daphnia magna & 48 & 12.90 & & 1 \\
\hline $\begin{array}{l}\text { 1-Propanaminium, N-(carboxymethyl)-N,N-dimethyl-3-[(1- } \\
\text { oxododecylamino]-, }\end{array}$ & $4292-10-8$ & 343.53 & 0.69 & $1.76 \mathrm{E}+03$ & & & & & 4 \\
\hline 2-(Dimethylamino)ethyl acrylate & $2439-35-2$ & & 0.68 & $2.40 \mathrm{E}+04$ & Daphnia magna & 48 & & 9.92 & 1 \\
\hline 2-Oxepanone & $502-44-3$ & & 0.68 & miscible & Daphnia magna & 48 & & 204.00 & 1 \\
\hline 2-Propenenitrile, 2-methyl- & $126-98-7$ & & 0.68 & $2.90 \mathrm{E}+04$ & Daphnia magna & 48 & & 200.00 & 1 \\
\hline Butane, 1,2-ероху- & $106-88-7$ & & 0.68 & $5.90 \mathrm{E}+04$ & Daphnia magna & 24 & & 159.70 & 1 \\
\hline Diaminotoluene & $25376-45-8$ & & 0.66 & $1.39 \mathrm{E}+01$ & Daphnia magna & 48 & & 1.73 & 1 \\
\hline Toluene-3,4-diamine & $496-72-0$ & & 0.66 & $1.39 \mathrm{E}+04$ & Daphnia magna & 48 & & 1.73 & 1 \\
\hline 3-Buten-2-ol, 2-methyl- & $115-18-4$ & & 0.66 & $1.90 \mathrm{E}+05$ & Daphnia magna Straus & 48 & & $>500$ & 3 \\
\hline 2-Butanone, oxime & $96-29-7$ & & 0.65 & $1.10 \mathrm{E}+05$ & & & & & 4 \\
\hline $\begin{array}{l}\text { Benzenesulfonic acid, 2,2'-(1,2-ethenediyl)bis[5-[[4-[bis(2- } \\
\text { hydroxyethyl)amino]-6-(phenylamino)-1,3,5-triazin-2-yl]amino-- }\end{array}$ & 4404-43-7 & 960.95 & 0.65 & & & & & & 4 \\
\hline
\end{tabular}


(Table S6. continued)

\begin{tabular}{|c|c|c|c|c|c|c|c|c|c|}
\hline Chemicals name & CAS Reg. no & $\begin{array}{c}\text { Molecular } \\
\text { weight } \\
\left(\mathrm{g} \mathrm{mol}^{-1}\right)\end{array}$ & $\log \mathrm{K}_{\mathrm{ow}}$ & Solubility $\left(\mathrm{mg} \mathrm{L}^{-1}\right)$ & Species & $\begin{array}{l}\text { Duration } \\
\text { (h) }\end{array}$ & $\begin{array}{c}\text { Measured } \\
\mathrm{EC}_{50} \\
\left(\mathrm{mg} \mathrm{L}^{-1}\right) \\
\end{array}$ & $\begin{array}{c}\text { Nominal } \\
\mathrm{EC}_{50} \\
\left(\mathrm{mg} \mathrm{L}^{-1}\right) \\
\end{array}$ & Classification \\
\hline Carbonochloridic acid, ethyl ester & $541-41-3$ & 108.53 & 0.63 & $3.21 \mathrm{E}+04$ & & & & & 4 \\
\hline Butan-2-ol & $78-92-2$ & & 0.61 & $4.50 \mathrm{E}+05$ & Daphnia magna & 48 & & 4227.00 & 1 \\
\hline Propanal & $123-38-6$ & 58.08 & 0.59 & $2.20 \mathrm{E}+05$ & Daphnia magna & 48 & & 125.00 & 1 \\
\hline \multirow[t]{2}{*}{ Silane, dimethoxydimethyl- } & $1112-39-6$ & 50.00 & 0.59 & $6.80 \mathrm{E}+03$ & Daphnia magna & 48 & & $>100$ & 3 \\
\hline & & & & & Daphnia magna & $\begin{array}{l}40 \\
48\end{array}$ & & 211.00 & 3 \\
\hline \multirow[t]{2}{*}{ Phthalonitrile } & $91-15-6$ & & 0.58 & $5.60 \mathrm{E}+02$ & Daphnia magna & 24 & & 330.00 & 1 \\
\hline & & & & & Daphnia magna & 48 & & 219.00 & \\
\hline \multirow{3}{*}{ 2,4-Pentanediol, 2-methyl- } & & & & & Daphnia magna & 48 & & 3200.00 & \\
\hline & $107-41-5$ & & 0.58 & miscible & Daphnia pulex & 48 & & 3300.00 & 1 \\
\hline & & & & & Daphnia magna & 48 & & 56.00 & \\
\hline \multirow[t]{2}{*}{ Diethylamine } & $109-89-7$ & 73.14 & 0.58 & miscible & Daphnia magna & 48 & & 58.00 & 1 \\
\hline & & & & & Daphnia magna & 48 & & 100.00 & \\
\hline Dibutyl phosphate & $107-66-4$ & & 0.57 & $1.70 \mathrm{E}+04$ & Daphnia magna & 24 & & 210.00 & 1 \\
\hline Ethanol, 2-(butoxyethoxy)- & $112-34-5$ & 162.23 & 0.56 & miscible & $\begin{array}{l}\text { Daphnia magna } \\
\text { Daphnia magna }\end{array}$ & 24 & & $\begin{array}{l}2850.00 \\
3200.00\end{array}$ & 1 \\
\hline \multirow{2}{*}{ Silane, (3-chloropropyl)trimethoxy- } & $2530-87-2$ & 19900 & 056 & $650 \mathrm{~F}+05$ & Daphnia magna & 48 & 869.00 & & 1 \\
\hline & $2530-87-2$ & 199.00 & 0.56 & $6.50 \mathrm{E}+05$ & Daphnia magna & 48 & 124.00 & & 1 \\
\hline \multirow{3}{*}{$\begin{array}{l}\text { Silane, trimethoxyphenyl- } \\
\text { 2-Butenal, 3-methyl- } \\
\text { Triethyleneglycol, monobutylether }\end{array}$} & 2996-92-1 & & 0.55 & $6.90 \mathrm{E}+04$ & Daphnia magna & 48 & $>0.0029$ & & 3 \\
\hline & $107-86-8$ & & 0.53 & $1.10 \mathrm{E}+05$ & Daphnia magna & 48 & & 13.50 & 1 \\
\hline & $143-22-6$ & & 0.51 & $1.00 \mathrm{E}+06$ & & & & & 4 \\
\hline Propanol, [(1-methyl-1,2-ethanediyl)bis(oxy)]bis- & 24800-44-0 & & $\begin{array}{l}0.50- \\
0.60\end{array}$ & & Daphnia magna & 24 & $>1000$ & & 3 \\
\hline \multirow{2}{*}{ Hydroquinone } & $123-31-9$ & & $0.50-$ & $7.30 \mathrm{E}+04$ & Daphnia magna & 24 & & 0.12 & 1 \\
\hline & $110-63-4$ & & $\begin{array}{l}0.61 \\
0.50\end{array}$ & $1.00 \mathrm{E}+05$ & Daphnia magna & 48 & & $>1000$ & 3 \\
\hline 1,4-Butanediol & & & & & Daphnia magna & 24 & & 765.00 & \\
\hline Acrylic acid & $79-10-7$ & & 0.46 & miscible & $\begin{array}{l}\text { Daphnia magna } \\
\text { Daphnia mana }\end{array}$ & $\begin{array}{l}48 \\
48\end{array}$ & $\begin{array}{l}95.00 \\
47.00\end{array}$ & & 1 \\
\hline \multirow{2}{*}{$\begin{array}{l}\text { Tetrahydrofuran } \\
\text { Maleic acid }\end{array}$} & $109-99-9$ & 72.11 & 0.46 & & Daphnia magna & & & & 4 \\
\hline & $110-16-7$ & 116.07 & 0.46 & $4.07 \mathrm{E}+05$ & & & & & 4 \\
\hline Oxirane, chloromethyl- & $106-89-8$ & & 0.45 & $6.60 \mathrm{E}+04$ & Daphnia magna & 24 & & 30.00 & 1 \\
\hline \multirow{2}{*}{$\begin{array}{l}\text { Propionic acid, 3-mercapto- } \\
\text { 2-Hydroxyethyl methacrylate }\end{array}$} & $107-96-0$ & & 0.43 & $6.03 \mathrm{E}+05$ & Daphnia magna & 48 & 9.00 & 40.00 & 1 \\
\hline & $868-77-9$ & & 0.42 & & Daphnia magna & 48 & & 380.00 & 1 \\
\hline 2-Furaldehyde & $98-01-1$ & & 0.41 & $8.30 \mathrm{E}+04$ & Daphnia magna & 72 & & $\begin{array}{r}13.00 \\
29.00\end{array}$ & 1 \\
\hline \multirow{2}{*}{$\mathrm{N}$-Vinyl-pyrrolidinone } & & & & & $\begin{array}{l}\text { Daphnia sp. } \\
\text { Dana }\end{array}$ & 24 & 130.00 & & 1 \\
\hline & $88-12-0$ & & 0.40 & & Daphnia sp. & 48 & 45.00 & & 1 \\
\hline $\begin{array}{l}\text { Ether, methyl vinyl } \\
\text { tert-Butylamine }\end{array}$ & $\begin{array}{c}107-25-5 \\
75-64-9\end{array}$ & 58.08 & $\begin{array}{l}0.40 \\
0.40\end{array}$ & $1.71 \mathrm{E}+04$ & & & & & $\begin{array}{l}4 \\
4\end{array}$ \\
\hline 2-Propanol, 1-methoxy-, acetate & $108-65-6$ & & 0.36 & $1.00 \mathrm{E}+05$ & Daphnia magna & 48 & & 373.00 & 1 \\
\hline Ethanol, 2-tert-butoxy- & $7580-85-0$ & 118.17 & 0.36 & $1.00 \mathrm{E}+05$ & $\begin{array}{l}\text { Dappnia magna } \\
\text { Daphnia magna }\end{array}$ & $\begin{array}{l}24 \\
48\end{array}$ & & $>>500$ & 3 \\
\hline Acrylic acid, monoester with 1,2-propanediol & $25584-83-2$ & 130.14 & 0.35 & $3.07 \mathrm{E}+05$ & Daphnia magna & 48 & & 24.00 & 1 \\
\hline & & & & & Daphnia magna & 24 & & 52.00 & \\
\hline Formaldehyde & $50-00-0$ & & 0.35 & & Daphnia pulex & 48 & & 5.80 & 1 \\
\hline & & & & & Daphnia magna & 24 & & 42.00 & \\
\hline & & & $0.34-$ & & Daphnia magna & 48 & & 34.40 & \\
\hline 2,4-Pentanedione & $123-54-6$ & 100.12 & 0.40 & $1.66 \mathrm{E}+05$ & Daphnia magna & 48 & & 47.60 & 1 \\
\hline Propane, 1-(allyloxy)-2,3-epoxy- & $106-92-3$ & & 0.34 & $1.28 \mathrm{E}+05$ & Daphnia magna & 48 & & 50.00 & 1 \\
\hline Propanal, 3-(methylthio)- & $3268-49-3$ & & 0.34 & $7.79 \mathrm{E}+04$ & Daphnia magna & 24 & & 25.00 & 1 \\
\hline Propanoic acid, anhydride & $123-62-6$ & & 0.33 & & $\begin{array}{l}\text { Daphnia magna } \\
\text { Daphnia magna }\end{array}$ & $\begin{array}{l}48 \\
48\end{array}$ & 4.50 & 22.70 & 1 \\
\hline Propionic acid & $79-09-4$ & & 0.33 & miscible & Daphnia magna & 48 & & 22.70 & 1 \\
\hline 2-(2-Ethoxyethoxy)ethyl acetate & $112-15-2$ & 176.21 & 0.32 & $1.00 \mathrm{E}+06$ & & & & & 4 \\
\hline 3-Butyn-2-ol, 2-methyl- & $115-19-5$ & & 0.32 & miscible & Daphnia magna & 48 & & $>500$ & 3 \\
\hline Propionitrile, 3-(triethoxysilyl)- & $919-31-3$ & & 0.32 & $1.00 \mathrm{E}+06$ & Daphnia magna & 48 & $>100$ & & 3 \\
\hline Pentaerythritol & $115-77-5$ & & 0.30 & $2.50 \mathrm{E}+04$ & Daphnia magna & 48 & & 600.00 & 1 \\
\hline Isocyanuric acid & $108-80-5$ & & 0.30 & $2.70 \mathrm{E}+03$ & Daphnia magna & 48 & & 1000.00 & 1 \\
\hline 2-Butanone & $78-93-3$ & 72.11 & 0.29 & $2.76 \mathrm{E}+05$ & Daphnia magna & 48 & & 5091.00 & 1 \\
\hline
\end{tabular}




\section{(Table S6. continued)}

\begin{tabular}{|c|c|c|c|c|c|c|c|c|c|}
\hline Chemicals name & CAS Reg. no & $\begin{array}{c}\text { Molecular } \\
\text { weight } \\
\left(\mathrm{g} \mathrm{mol}^{-1}\right)\end{array}$ & $\log \mathrm{K}_{\mathrm{ow}}$ & Solubility $\left(\mathrm{mg} \mathrm{L}^{-1}\right)$ & Species & $\begin{array}{l}\text { Duration } \\
\text { (h) }\end{array}$ & $\begin{array}{c}\text { Measured } \\
\mathrm{EC}_{50} \\
\left(\mathrm{mg} \mathrm{L}^{-1}\right)\end{array}$ & $\begin{array}{c}\text { Nominal } \\
\mathrm{EC}_{50} \\
\left(\mathrm{mg} \mathrm{L}^{-1}\right)\end{array}$ & Classification \\
\hline Acetic acid, mercapto- & $68-11-1$ & & 0.27 & $>1.00 \mathrm{E}+06$ & Daphnia magna & 48 & & 38.00 & 1 \\
\hline 2-Propanamine & $75-31-0$ & & 0.26 & & Daphnia magna & 48 & & 47.40 & 1 \\
\hline Ethyl acetoacetate & $141-97-9$ & & 0.25 & $1.25 \mathrm{E}+05$ & Daphnia magna & 24 & & $790-800$ & 1 \\
\hline Silanetriol, methyl-, triacetate & $4253-34-3$ & 220.00 & 0.25 & $9.10 \mathrm{E}+04$ & $\begin{array}{l}\text { Daphnia magna } \\
\text { Daphnia magna }\end{array}$ & $\begin{array}{l}48 \\
48\end{array}$ & & $\begin{array}{l}62.00 \\
6000.00\end{array}$ & 1 \\
\hline 1-Propanol & $71-23-8$ & & 0.25 & & & & & & 4 \\
\hline Trimethylamine & $75-50-3$ & & 0.25 & $4.10 \mathrm{E}+05$ & Daphnia magna & 48 & & 139.95 & 1 \\
\hline 4-Piperidinol, 2,2,6,6-tetramethyl- & $2403-88-5$ & & 0.24 & $1.00 \mathrm{E}+05$ & Daphnia magna & $\begin{array}{l}24 \\
48\end{array}$ & & $\begin{array}{l}130.10 \\
100.10\end{array}$ & 1 \\
\hline Dimethyl carbonate & $616-38-6$ & 90.08 & 0.23 & & Daphnia magna & & & & 4 \\
\hline Chloroacetic acid & $79-11-8$ & 94.50 & 0.22 & $4.26 \mathrm{E}+03$ & $\begin{array}{l}\text { Daphnia magna } \\
\text { Daphnia magna }\end{array}$ & 48 & & $\begin{array}{c}75.00 \\
180.00\end{array}$ & 1 \\
\hline & & & & & Daphnia magna & 48 & & 165.00 & \\
\hline Ethanol, 2-(diethylamino)- & $100-37-8$ & & 0.21 & miscible & $\begin{array}{l}\text { Daphnia magna } \\
\text { Dapnagian }\end{array}$ & 48 & & 92.40 & 1 \\
\hline Difluoromethane & $75-10-5$ & & 0.21 & $4.40 \mathrm{E}+03$ & & & & & 4 \\
\hline Acetin, tri- & $102-76-1$ & & 0.21 & $7.00 \mathrm{E}+04$ & $\begin{array}{l}\text { Daphnia magna } \\
\text { Daphnia magna }\end{array}$ & $\begin{array}{l}48 \\
48\end{array}$ & $\begin{array}{l}768.00 \\
810.90\end{array}$ & & 1 \\
\hline Nitric acid & $7697-37-2$ & 62.00 & 0.21 & very soluble & & & & & 4 \\
\hline Methyl acetate & $79-20-9$ & 74.08 & 0.18 & $2.50 \mathrm{E}+05-2.95 \mathrm{E}+05$ & Daphnia magna & 48 & 1027.00 & & 1 \\
\hline m-Phenylene-bis(methylamine) & $1477-55-0$ & & 0.18 & $1.00 \mathrm{E}+05$ & Daphnia magna & 48 & $\begin{array}{l}15.20 \\
1600\end{array}$ & & 1 \\
\hline Stannane, butyltrichloro- & $1118-46-3$ & & 0.18 & $1.00 \mathrm{E}+03-1.00 \mathrm{E}+04$ & Daphnia magna & 48 & $\begin{array}{l}10.00 \\
83.00\end{array}$ & & 1 \\
\hline Butanol, 3-methoxy-3-methyl- & $56539-66-3$ & & 0.18 & $1.00 \mathrm{E}+05$ & Daphnia magna & 48 & & $>1000$ & 3 \\
\hline Ethane, nitro- & $79-24-3$ & & 0.18 & $4.50 \mathrm{E}+04$ & Daphnia magna & 24 & 1200.00 & & 1 \\
\hline 2-Propen-1-ol & $107-18-6$ & 58.08 & 0.17 & miscible & $\begin{array}{l}\text { Daphnia magna } \\
\text { Ophryotrocha diadema }\end{array}$ & $\begin{array}{l}48 \\
48\end{array}$ & $\begin{array}{c}2.10 \\
0.33-1.0\end{array}$ & & 1 \\
\hline Dimethyl sulfate & $77-78-1$ & & 0.16 & $2.80 \mathrm{E}+04$ & Daphnia magna & 48 & & 17.00 & 1 \\
\hline Formic acid, chloro-, methyl ester & $79-22-1$ & & 0.14 & $9.28 \mathrm{E}+05$ & & & & & 4 \\
\hline 1,3-Propanediol, 2,2-dimethyl- & $126-30-7$ & 104.15 & 0.12 & $1.90 \mathrm{E}+03$ & Daphnia magna & 48 & & $>1000$ & 3 \\
\hline 2H-Azepin-2-one, hexahydro- & $105-60-2$ & & 0.12 & $4.56 \mathrm{E}+06$ & Daphnia magna & 48 & & 2430.00 & 1 \\
\hline Adipic acid & $124-04-9$ & & 0.09 & $2.30 \mathrm{E}+04$ & Daphnia magna & 48 & & 85.60 & 1 \\
\hline Cyclohexanone & $108-94-1$ & 98.14 & 0.08 & $2.30 \mathrm{E}+04$ & $\begin{array}{l}\text { Daphnia magna } \\
\text { Daphnia magna Straus }\end{array}$ & 24 & & 800.00 & 1 \\
\hline Benzenesulfonic acid, 44'-oxybis- dihydrazide & $80-51-3$ & & 0.08 & $625 \mathrm{~F}+01$ & Daphnia magna & 24 & & $\begin{array}{l}820.00 \\
15.00\end{array}$ & 1 \\
\hline Benzenesuronic acia, 4,4-oxyois", anyourazide & $80-51-3$ & & 0.08 & $0.25 \mathrm{E}+01$ & Daphnia magna & & 2.90 & & 1 \\
\hline Morpholine, 4-ethyl- & $100-74-3$ & & 0.08 & $3.03 \mathrm{E}+05$ & $\begin{array}{l}\text { Daphnia magna } \\
\text { Daphnia magna }\end{array}$ & 48 & $>92$ & $>580$ & 3 \\
\hline Ethanol, 2-propoxy- & 2807-30-9 & & 0.08 & completely soluble & Daphnia magna & 48 & & $>5000$ & 3 \\
\hline Toluene, 2,4-diamine & $95-80-7$ & & 0.07 & $3.80 \mathrm{E}+04$ & $\begin{array}{l}\text { Daphnia magna } \\
\text { Daphnia magna }\end{array}$ & $\begin{array}{l}24 \\
48\end{array}$ & $\begin{array}{c}12.00 \\
1.60\end{array}$ & & 1 \\
\hline Phosphoryl trichloride & $10025-87-3$ & 153.33 & 0.06 & & Daphnia magna & 48 & & 35.40 & 1 \\
\hline Oxirane, methyl- & $75-56-9$ & 58.08 & 0.06 & $3.95 \mathrm{E}+05-4.05 \mathrm{E}+05$ & Daphnia magna & 48 & & 350.00 & 1 \\
\hline 2-Propanol & $67-63-0$ & & 0.05 & $1.00 \mathrm{E}+06$ & Daphnia magna & 24 & & $>10000$ & 3 \\
\hline Phenol, 4-amino- & $123-30-8$ & & 0.04 & $1.57 \mathrm{E}+04$ & Daphnia magna & 48 & 0.10 & & 1 \\
\hline Silicic acid, (H4SiO4), tetraethyl ester & $78-10-4$ & & 0.04 & & $\begin{array}{l}\text { Daphnia magna } \\
\text { Daphnia magna }\end{array}$ & $\begin{array}{l}48 \\
48\end{array}$ & $\begin{array}{l}>75 \\
>844\end{array}$ & & 3 \\
\hline Ethanol, 2-(1-methylethoxy)- & 109-59-1 & & 0.04 & $1.00 \mathrm{E}+05$ & $\begin{array}{l}\text { Daphnia magna } \\
\text { Daphnia magna }\end{array}$ & $\begin{array}{l}48 \\
48\end{array}$ & $>844$ & & 3 \\
\hline $\begin{array}{l}\text { 1,2-Benzenedicarboxylic acid, bis (2-methoxyethyl) ester } \\
\text { (Di(methoxyethyl)phthalate) }\end{array}$ & $117-82-8$ & & 0.04 & $8.50 \mathrm{E}+00$ & & & & & 4 \\
\hline Methyl formate & $107-31-3$ & & 0.03 & $2.40 \mathrm{E}+04$ & $\begin{array}{l}\text { Daphnia magna } \\
\text { Chaetogammarus marinus }\end{array}$ & $\begin{array}{l}48 \\
96\end{array}$ & & $\begin{array}{l}>500 \\
320-560\end{array}$ & 1 \\
\hline 1,6-Hexanediamine & $124-09-4$ & 116.24 & 0.02 & $8.00 \mathrm{E}+02$ & $\begin{array}{l}\text { Chaetogammarus marnus } \\
\text { Daphnia magna }\end{array}$ & $\begin{array}{l}96 \\
48\end{array}$ & & $\begin{aligned} 320-560 \\
23.40\end{aligned}$ & 1 \\
\hline Toluene-2,3-diamine & $2687-25-4$ & 122.17 & 0.01 & $1.39 \mathrm{E}+04$ & & & & & 4 \\
\hline Propanol, 1(or 2)-(2-methoxymethylethoxy)- & $34590-94-8$ & & 0.01 & & & & & & 4 \\
\hline $\begin{array}{l}\text { Chlorosulfuric acid } \\
\text { Theophylline }\end{array}$ & $\begin{array}{c}7790-94-5 \\
58-55-9\end{array}$ & 116.52 & 0.00 & & & & & & 4 \\
\hline Theophylline & $58-55-9$ & & -0.01 & $5.50 \mathrm{E}+03-8.30 \mathrm{E}+03$ & Daphnia magna & 48 & & 178.00 & 1 \\
\hline Acrolein & $107-02-8$ & 56.06 & -0.01 & $2.06 \mathrm{E}+05-2.70 \mathrm{E}+05$ & $\begin{array}{l}\text { Daphnia magna } \\
\text { Daphnia magna }\end{array}$ & $\begin{array}{l}48 \\
48\end{array}$ & 0.05 & 0.06 & 1 \\
\hline Glutaraldehyde & $111-30-8$ & & -0.01 & miscible & $\begin{array}{l}\text { Daphnia magna } \\
\text { Daphnia magna }\end{array}$ & $\begin{array}{l}48 \\
48\end{array}$ & & $\begin{array}{c}0.35 \\
16.30\end{array}$ & 1 \\
\hline
\end{tabular}


(Table S6. continued)

\begin{tabular}{|c|c|c|c|c|c|c|c|c|c|}
\hline Chemicals name & CAS Reg. no & $\begin{array}{c}\text { Molecular } \\
\text { weight } \\
\left(\mathrm{g} \mathrm{mol}^{-1}\right) \\
\end{array}$ & $\log \mathrm{K}_{\mathrm{ow}}$ & Solubility $\left(\mathrm{mg} \mathrm{L}^{-1}\right)$ & Species & $\begin{array}{l}\text { Duration } \\
\text { (h) }\end{array}$ & $\begin{array}{c}\text { Measured } \\
\mathrm{EC}_{50} \\
\left(\mathrm{mg} \mathrm{L}^{-1}\right) \\
\end{array}$ & $\begin{array}{c}\text { Nominal } \\
E_{50} \\
\left(\mathrm{mg} \mathrm{L}^{-1}\right) \\
\end{array}$ & Classification \\
\hline Phosphonic acid, (1-hydroxyethylidene)bis- & $2809-21-4$ & \multirow[t]{4}{*}{206.03} & -0.01 & $6.90 \mathrm{E}+05$ & $\begin{array}{l}\text { Daphnia magna } \\
\text { Daphnia magna } \\
\text { Daphnia magna }\end{array}$ & $\begin{array}{l}24 \\
48 \\
48\end{array}$ & & $\begin{array}{l}165.00 \\
166.70 \\
527.00\end{array}$ & 1 \\
\hline 1H-Imidazole & $288-32-4$ & & -0.02 & $6.63 \mathrm{E}+05$ & Daphnia magna & 48 & & 341.50 & 1 \\
\hline 1,5-Pentanediol, 3-methyl- & 4457-71-0 & & -0.03 & miscible & Daphnia magna & 48 & & $>1000$ & 3 \\
\hline Propanenitrile, 2-hydroxy-2-methyl- & $75-86-5$ & & -0.03 & miscible & Daphnia magna & 48 & & 0.13 & 1 \\
\hline Silane, ethenyltrimethoxy- & $2768-02-7$ & 148.23 & -0.03 & $5.04 \mathrm{E}+05$ & $\begin{array}{l}\text { Daphnia magna } \\
\text { Daphnia magna }\end{array}$ & $\begin{array}{l}48 \\
48\end{array}$ & & $\begin{array}{l}168.70 \\
>100\end{array}$ & 1 \\
\hline Dimethyl malonate & $108-59-8$ & 132.12 & -0.05 & $9.90 \mathrm{E}+04$ & Daphnia magna & 48 & & $>1000$ & 3 \\
\hline Ethanol, 2-mercapto- & $60-24-2$ & 78.13 & -0.06 & miscible & $\begin{array}{l}\text { Daphnia magna } \\
\text { Daphnia magna }\end{array}$ & $\begin{array}{l}48 \\
48\end{array}$ & \multirow{2}{*}{$\begin{array}{l}0.40 \\
1.00\end{array}$} & & 1 \\
\hline $\begin{array}{l}\text { Caffeine } \\
\text { 2-Furanmethanol, tetrahydro- }\end{array}$ & $\begin{array}{l}58-08-2 \\
97-99-4\end{array}$ & $\begin{array}{l}194.19 \\
102.13\end{array}$ & $\begin{array}{l}-0.09 \\
-0.11\end{array}$ & \multirow[t]{2}{*}{$2.50 \mathrm{E}+05$} & Daphnia magna & 48 & & 182.00 & $\begin{array}{l}2 \\
4\end{array}$ \\
\hline Ethanamine & 75-04-7 & & -0.13 & & Ceriodaphnia dubia & 48 & \multirow[t]{2}{*}{7.90} & & 1 \\
\hline Acetaldehyde, oxime & $107-29-9$ & 59.07 & -0.13 & $>1.00 \mathrm{E}+04$ & Daphnia magna & 48 & & 0.40 & 1 \\
\hline Diacetone alcohol & $123-42-2$ & 116.16 & -0.14 & $1.00 \mathrm{E}+05$ & Daphnia magna & 48 & & $>1000$ & 3 \\
\hline 2-Propanol, 1,1',1"-nitrilotri- & $122-20-3$ & 191.27 & -0.15 & miscible & $\begin{array}{l}\text { Daphnia magna } \\
\text { Daphnia magna }\end{array}$ & $\begin{array}{l}48 \\
48\end{array}$ & & $\begin{array}{c}857.00 \\
>500\end{array}$ & 1 \\
\hline 2-Propenamide, 2-methyl- & $79-39-0$ & 85.10 & -0.15 & \multirow{3}{*}{$\begin{array}{l}1.000+05 \\
8.00 \mathrm{E}+02\end{array}$} & Daphnia magna & 48 & & $>1000$ & 3 \\
\hline Undecanoic acid, 11-amino- & $2432-99-7$ & 201.31 & -0.16 & & Daphnia magna & 48 & \multirow[t]{2}{*}{$>350$} & 1000 & 3 \\
\hline 2-Propanol, 1-[2-(2-methoxy-1-methylethoxy)-1-methylethoxy]- & 20324-33-8 & 206.28 & -0.20 & & & 然 & & & 4 \\
\hline Ethanol, 2-(2-propoxyethoxy)- & 6881-94-3 & 148.20 & -0.20 & \multirow[t]{2}{*}{$1.00 \mathrm{E}+06$} & Daphnia magna & 48 & \multirow{3}{*}{0.78} & $>10000$ & 3 \\
\hline 2-Hydroxyethyl acrylate & $818-61-1$ & 116.12 & -0.21 & & Daphnia magna & 48 & & & 1 \\
\hline 2,3-Dibromosuccinic acid & $526-78-3$ & & -0.21 & $2.00 \mathrm{E}+04$ & & & & & 4 \\
\hline Acetone & $67-64-1$ & 58.08 & -0.24 & $1.00 \mathrm{E}+06$ & $\begin{array}{l}\text { Daphnia magna } \\
\text { Daphnia pulex }\end{array}$ & $\begin{array}{l}48 \\
48\end{array}$ & & $\begin{array}{l}12600.00 \\
8800.00\end{array}$ & 1 \\
\hline 1,4-Dioxane & $123-91-1$ & 88.11 & -0.27 & miscible & $\begin{array}{l}\text { Daphnia magna } \\
\text { Daphnia magna }\end{array}$ & $\begin{array}{l}24 \\
24\end{array}$ & & $\begin{array}{l}4700.00 \\
8450.00\end{array}$ & 1 \\
\hline Ethanol & $64-17-5$ & 46.07 & -0.31 & miscible & Daphnia magna & 48 & & 12340.00 & 1 \\
\hline 1,4-Dicyanobutane & $111-69-3$ & 108.14 & -0.32 & $8.30 \mathrm{E}+04$ & Daphnia magna & 24 & & 445.00 & 1 \\
\hline Propanenitrile, 2-hydroxy- & $78-97-7$ & & -0.32 & infinite & Daphnia magna & 48 & & 17.00 & 1 \\
\hline Methane, nitro- & $75-52-5$ & & -0.33 & $1.11 \mathrm{E}+05$ & Daphnia magna & 24 & & 450.00 & 1 \\
\hline Butanedioic acid, methylene- & $97-65-4$ & & -0.34 & & Daphnia magna & 24 & 240.00 & & 1 \\
\hline 1,2-Butylene glycol & $584-03-2$ & 90.14 & -0.34 & $1.00 \mathrm{E}+05$ & Daphnia magna & 48 & & $>1000$ & 3 \\
\hline Acetonitrile & $75-05-8$ & 41.05 & -0.34 & $1.00 \mathrm{E}+06$ & $\begin{array}{l}\text { Daphnia magna } \\
\text { Dapnia pulex }\end{array}$ & $\begin{array}{l}24 \\
18\end{array}$ & & $\begin{array}{l}>10,000 \\
5838.00\end{array}$ & 1 \\
\hline Oxydiethylene chloroformate & $106-75-2$ & & -0.34 & $3.00 \mathrm{E}+00$ & Dapniapulex & & & & 4 \\
\hline Propane, 1-Amino-3-dimethylamino- & $109-55-7$ & & -0.35 & miscible & Daphnia magna & 48 & & 59.50 & 1 \\
\hline Nicotinamide & $98-92-0$ & 122.13 & -0.38 & $6.91 \mathrm{E}+05-1.00 \mathrm{E}+06$ & Daphnia magna & 24 & $>1000$ & (1..00 & 3 \\
\hline Dimethylamine & $124-40-3$ & 45.08 & -0.38 & 年. & 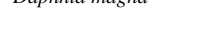 & 27 & 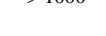 & & 4 \\
\hline Propylamine, 3-methoxy- & 5332-73-0 & 89.14 & -0.42 & & & & & & 4 \\
\hline 2-Propanol, 1-methoxy- & $107-98-2$ & 90.12 & -0.44 & $2.00 \mathrm{E}+05$ & $\begin{array}{l}\text { Daphnia magna } \\
\text { Daphnia magna }\end{array}$ & $\begin{array}{l}48 \\
48\end{array}$ & & $\begin{array}{l}>500 \\
>23300\end{array}$ & 3 \\
\hline & & & & & Daphnia magna & 24 & $>1000$ & & \\
\hline & & & & & Daphnia magna & 48 & 4897.00 & & \\
\hline 2-Pyrrolidinone 1-methyl- & $872-50-4$ & 99.13 & -0.46 & $100 \mathrm{E}+06$ & Daphnia magna & 48 & 1.23 & & 1 \\
\hline 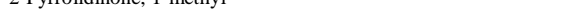 & 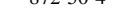 & (15 & -0.40 & $1.000+00$ & Gammarus sp. & 48 & 4655.00 & & 1 \\
\hline & & & & & Neopanope texana sayi & 96 & 1585.00 & & \\
\hline & & & & & Palaemonetes vulgaris & 96 & 1107.00 & & \\
\hline Trimethyl phosphate & $512-56-1$ & & -0.46 & miscible & Daphnia magna & 24 & & $>1,000$ & 3 \\
\hline 1,3-Propanediol, 2-ethyl-2-(hydroxymethyl)- & $77-99-6$ & 134.20 & -0.47 & $>1.00 \mathrm{E}+05$ & $\begin{array}{l}\text { Daphnia magna } \\
\text { Daphnia magna }\end{array}$ & $\begin{array}{l}24 \\
48\end{array}$ & & $\begin{array}{l}>1000 \\
13000.00\end{array}$ & 1 \\
\hline Guanidine, cyano- & $461-58-5$ & 84.08 & -0.52 & $4.00 \mathrm{E}+04$ & Daphnia magna & 24 & $>1000$ & & 3 \\
\hline & & & & & Daphnia magna & 48 & & 0.73 & \\
\hline Peroxyacetic acid & $79-21-0$ & 76.05 & -0.52 & $1.00 \mathrm{E}+06$ & Daphnia magna & 48 & & 1.10 & 1 \\
\hline & & & & & Daphnia magna & 48 & $10200-3$ & 0.50 & \\
\hline Ethaneamine, 2'-oxibis[N,N-dimethyl-] & $3033-62-3$ & 160.26 & -0.54 & $1.00 \mathrm{E}+06$ & $\begin{array}{l}\text { Daphnia magna } \\
\text { Daphizi magna }\end{array}$ & $\begin{array}{l}48 \\
48\end{array}$ & 102.00 & & 1 \\
\hline Formic acid & $64-18-6$ & 46.03 & -0.54 & miscible & $\begin{array}{l}\text { Daphnia magna } \\
\text { Carcinus maenas }\end{array}$ & $\begin{array}{l}48 \\
48\end{array}$ & & $\begin{array}{r}32.19 \\
80-90\end{array}$ & 1 \\
\hline 1 & (3) & (1) & 0.01 & moverie & Daphnia magna & 48 & & 151.20 & 1 \\
\hline
\end{tabular}


(Table S6. continued)

\begin{tabular}{|c|c|c|c|c|c|c|c|c|c|}
\hline Chemicals name & CAS Reg. no & $\begin{array}{c}\text { Molecular } \\
\text { weight } \\
\left(\mathrm{g} \mathrm{mol}^{-1}\right)\end{array}$ & $\log \mathrm{K}_{\mathrm{ow}}$ & Solubility $\left(\mathrm{mg} \mathrm{L}^{-1}\right)$ & Species & $\begin{array}{l}\text { Duration } \\
\text { (h) }\end{array}$ & $\begin{array}{c}\text { Measured } \\
\mathrm{EC}_{50} \\
\left(\mathrm{mg} \mathrm{L}^{-1}\right)\end{array}$ & $\begin{array}{c}\text { Nominal } \\
\mathrm{EC}_{50} \\
\left(\mathrm{mg} \mathrm{L}^{-1}\right) \\
\end{array}$ & Classification \\
\hline Methanamine & $74-89-5$ & 31.06 & -0.57 & $1.00 \mathrm{E}+06$ & Daphnia magna & 48 & & 702.00 & 1 \\
\hline Acetic anhydride & $108-24-7$ & 102.09 & -0.58 & $2.60 \mathrm{E}+04$ & Daphnia magna & 24 & & 55.00 & 1 \\
\hline Nicotinic acid & $59-67-6$ & 123.11 & -0.59 & & Daphnia magna & 48 & & 77.00 & 1 \\
\hline Lactic acid & $50-21-5$ & 90.10 & -0.62 & $8.76 \mathrm{E}+05$ & Daphnia magna & 48 & & 240.00 & 1 \\
\hline 2-Propanol, 1,1'-oxydi- & $110-98-5$ & 134.18 & -0.64 & miscible & & & & & 4 \\
\hline m-Toluenesulfonic acid, 6-amino- & $88-44-8$ & 187.22 & -0.67 & $6.00 \mathrm{E}+03$ & Daphnia magna & 48 & $>10$ & & 3 \\
\hline Silane, trimethoxymethyl- & $1185-55-3$ & & -0.67 & $1.00 \mathrm{E}+06$ & Daphnia magna & 48 & $>122$ & & 3 \\
\hline DL-Pantoyl lactone & $79-50-5$ & 130.14 & -0.69 & $5.00 \mathrm{E}+02$ & Daphnia magna & 48 & & $>130$ & 3 \\
\hline Methyl acetoacetate & $105-45-3$ & & -0.69 & & & & & & 4 \\
\hline Ethanol, 2-(2-ethoxyethoxy)- & $111-90-0$ & 134.18 & -0.69 & $1.00 \mathrm{E}+06$ & Daphnia magna & 48 & & 3996.00 & 1 \\
\hline Silicic acid, ethyl ester & $11099-06-2$ & 106.15 & -0.72 & $1.00 \mathrm{E}+05$ & Daphnia magna & 48 & & $>193$ & 3 \\
\hline 2-Butyne-1,4-diol & $110-65-6$ & 86.09 & -0.73 & $7.50 \mathrm{E}+05$ & Daphnia magna Straus & 48 & & 26.80 & 1 \\
\hline Methanol & $67-56-1$ & 32.04 & -0.74 & miscible & Daphnia magna & 48 & & $>10,000$ & 3 \\
\hline Ethanol, 2,2'-thiodi- & $111-48-8$ & & -0.75 & miscible & Daphnia magna & 48 & & $>500$ & 3 \\
\hline Phosphoric acid & $7664-38-2$ & 98.00 & -0.77 & $5.48 \mathrm{E}+06$ & Daphnia magna & 48 & $>376$ & & 3 \\
\hline Thiophene, tetrahydro-, 1,1-dioxide & $126-33-0$ & 120.17 & -0.77 & $1.00 \mathrm{E}+05$ & $\begin{array}{l}\text { Daphnia magna } \\
\text { Daphnia magna }\end{array}$ & $\begin{array}{l}48 \\
48\end{array}$ & & $\begin{array}{l}852.00 \\
95.00\end{array}$ & 1 \\
\hline Acetamide, N,N-dimethyl- & $127-19-5$ & 87.12 & -0.77 & miscible & $\begin{array}{l}\text { Daphnia magna } \\
\text { Daphnia magna }\end{array}$ & $\begin{array}{l}24 \\
24 \\
48\end{array}$ & & $\begin{array}{l}>>500 \\
>500\end{array}$ & 3 \\
\hline Urea, 1,3-dimethyl- & $96-31-1$ & & -0.78 & $7.65 \mathrm{E}+05$ & Daphnia magna & 48 & $>500$ & & 3 \\
\hline 2-Propanol, 1,1'-iminodi- & $110-97-4$ & 133.19 & -0.79 & $4.00 \mathrm{E}+05$ & Daphnia magna & 48 & & 277.70 & 1 \\
\hline \multirow[t]{2}{*}{ Formamide } & $75-12-7$ & 45.04 & -0.82 & $1.00 \mathrm{E}+06$ & Daphnia magna & 48 & & $>500$ & 3 \\
\hline & & & & & Daphnia magna & 48 & $>100$ & & \\
\hline \multirow[t]{2}{*}{ Formamide, N,N-dimethyl- } & $68-12-2$ & 73.09 & -0.85 & miscible & Daphnia magna & 24 & $\begin{array}{l}19800.00 \\
150000\end{array}$ & & 1 \\
\hline & & & & & Daphnia magna & 48 & 15700.00 & & \\
\hline Stannane, trichloromethyl- & $993-16-8$ & 240.10 & -0.90 & $1.04 \mathrm{E}+06$ & $\begin{array}{l}\text { Daphnia magna } \\
\text { Daphnia magna }\end{array}$ & 48 & $>101$ & & 1 \\
\hline Urea, N,N"-(2-methylpropylidene)bis- & $6104-30-9$ & 174.20 & -0.90 & $300-3000$ & Daphnia magna & 48 & & 500.00 & 1 \\
\hline Ethanol, 2-(methylamino)- & $109-83-1$ & & -0.91 & $1.00 \mathrm{E}+06$ & Daphnia magna & 48 & 33.00 & & 1 \\
\hline Silane, trimethoxy[3-(oxiranylmethoxy)propyl]- & 2530-83-8 & 236.00 & -0.92 & $1.00 \mathrm{E}+06$ & $\begin{array}{l}\text { Daphnia magna } \\
\text { Daphnia magna }\end{array}$ & $\begin{array}{l}48 \\
48\end{array}$ & & $\begin{array}{l}710.00 \\
473.00\end{array}$ & 1 \\
\hline Ethanol, 2-(dimethylamino)- & $108-01-0$ & 89.14 & -0.94 & miscible & Daphnia magna & 48 & & 98.77 & 1 \\
\hline 1,3-Propanediol, 2-(hydroxymethyl)-2-methyl- & $77-85-0$ & & -0.95 & $3.00 \mathrm{E}+05$ & Daphnia magna Straus & $\begin{array}{l}48 \\
48\end{array}$ & & $\begin{array}{r}98.37 \\
>100\end{array}$ & 3 \\
\hline $\begin{array}{l}\text { Triethylene glycol, monoethyl ether } \\
\text { Ther }\end{array}$ & $112-50-5$ & 178.20 & $\begin{array}{l}-0.93 \\
-0.96\end{array}$ & 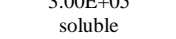 & $\begin{array}{l}\text { Daphnna magna } \\
\text { Daphnia magna }\end{array}$ & 48 & & $>10000$ & 3 \\
\hline 2-Propanol, 1-amino- & $78-96-6$ & 191.27 & -0.96 & & Daphnia magna & 48 & & 109.00 & 1 \\
\hline Triethanolamine & $102-71-6$ & 149.19 & -1.00 & & Daphnia magna & 24 & & 1390.00 & 1 \\
\hline \multirow{2}{*}{ Ethanol, 2,2'-(methylimino)di- } & $105-59-9$ & 119.16 & -1.08 & $1.00 \mathrm{E}+06$ & $\begin{array}{l}\text { Daphnia magna } \\
\text { Daphnia magna }\end{array}$ & $\begin{array}{l}48 \\
48\end{array}$ & & $\begin{array}{l}233.00 \\
230.00\end{array}$ & 1 \\
\hline & $280-57-9$ & & -1.13 & $6.10 \mathrm{E}+05$ & $\begin{array}{l}\text { Daphnia magna } \\
\text { Daphnia magna }\end{array}$ & $\begin{array}{l}48 \\
48\end{array}$ & & $\begin{array}{c}230.00 \\
232\end{array}$ & 3 \\
\hline Ethanol, 2-(2-methoxyethoxy)- & $111-77-3$ & 120.15 & -1.18 & & Daphnia magna & 48 & & 1192.00 & 1 \\
\hline Dimethyl phosphonate & $868-85-9$ & & -1.20 & $>1.00 \mathrm{E}+05$ & Daphnia magna & 48 & & 25.00 & 1 \\
\hline Silane, trimethoxy- & $2487-90-3$ & & -1.22 & $1.00 \mathrm{E}+06$ & Daphnia magna & 48 & & $>100$ & 3 \\
\hline Piperazine & $110-85-0$ & & -1.24 & $1.50 \mathrm{E}+05$ & Daphnia magna & 48 & & 21.00 & 1 \\
\hline Diethylenetriamine & $111-40-0$ & 103.20 & -1.30 & miscible & Daphnia magna & 48 & & 53.50 & 1 \\
\hline Dimethyl sulfoxide & $67-68-5$ & 78.13 & -1.35 & $1.00 \mathrm{E}+06$ & $\begin{array}{l}\text { Daphnia magna } \\
\text { Daphnia magna }\end{array}$ & $\begin{array}{l}48 \\
24\end{array}$ & \multirow{11}{*}{$\begin{array}{c}\text { low-level } \\
\text { toxicity }\end{array}$} & $\begin{array}{l}24600.00 \\
58200.00\end{array}$ & 1 \\
\hline Ethylene glycol & 107-21-1 & 62.07 & -1.36 & miscible & Daphnia magna Straus & 48 & & & 3 \\
\hline Melamine & $108-78-1$ & 126.12 & -1.37 & $3.10 \mathrm{E}+03$ & Daphnia magna & 48 & & $>2000$ & 3 \\
\hline Ammonia & 7664-41-7 & 17.03 & -1.38 & $8.90 \mathrm{E}+05$ & Daphnia magna & 48 & & 25.00 & 1 \\
\hline 7-Amino-4-hydroxy-2-naphthalenesulfonic acid & $87-02-5$ & & -1.39 & $5.00 \mathrm{E}+00$ & & & & & 4 \\
\hline Triethylene tetramine & $112-24-3$ & 146.23 & -1.40 & miscible & Daphnia magna & 48 & & 31.00 & 1 \\
\hline & $57-55-6$ & 76.09 & -1.41 & soluble & Daphnia magna & 48 & & 43500.00 & 1 \\
\hline \multirow[t]{2}{*}{ Ethanol, 2-(2-(2-methoxyethoxy)ethoxy)- } & $112-35-6$ & 164.20 & -1.46 & soluble & Daphnia magna & 48 & & $>10000$ & 3 \\
\hline & & & & & Daphnia magna & 48 & & 22.00 & \\
\hline \multirow{2}{*}{ Ethanol, 2-((2-aminoethyl)amino)- } & $111-41-1$ & 104.15 & -1.46 & unlimited & Daphnia magna & 48 & & 190.00 & 1 \\
\hline & $111-46-6$ & 106.12 & -1.47 & miscible & $\begin{array}{l}\text { Daphnia magna } \\
\text { Daphnia magna }\end{array}$ & $\begin{array}{l}48 \\
48\end{array}$ & & $\begin{array}{c}140.00 \\
48900.00\end{array}$ & 1 \\
\hline
\end{tabular}


(Table S6. continued)

\begin{tabular}{|c|c|c|c|c|c|c|c|c|c|}
\hline Chemicals name & CAS Reg. no & $\begin{array}{c}\text { Molecular } \\
\text { weight } \\
\left(\mathrm{g} \mathrm{mol}^{-1}\right)\end{array}$ & $\log K_{\text {ow }}$ & Solubility $\left(\mathrm{mg} \mathrm{L}^{-1}\right)$ & Species & $\begin{array}{l}\text { Duration } \\
\text { (h) }\end{array}$ & $\begin{array}{c}\text { Measured } \\
\mathrm{EC}_{50} \\
\left(\mathrm{mg} \mathrm{L}^{-1}\right)\end{array}$ & $\begin{array}{c}\text { Nominal } \\
\mathrm{EC}_{50} \\
\left(\mathrm{mg} \mathrm{L}^{-1}\right)\end{array}$ & Classification \\
\hline \multirow{2}{*}{ Piperazine, 1-(2-aminoethyl)- } & \multirow{2}{*}{$140-31-8$} & \multirow{2}{*}{129.20} & & \multirow{2}{*}{ miscible } & Daphnia magna & 48 & \multirow{3}{*}{35.40} & 32.00 & \multirow[b]{2}{*}{1} \\
\hline & & & -1.48 & & Daphnia magna & 24 & & 190.00 & \\
\hline Propanol, oxybis- & $25265-71-8$ & \multirow[b]{2}{*}{260.52} & -1.49 & \multirow[b]{2}{*}{ water reactive } & Daphnia magna & 48 & & \multirow[b]{2}{*}{$\begin{array}{c}21.60 \\
0.49\end{array}$} & 4 \\
\hline Stannane, tetrachloro- & $7646-78-8$ & & -1.53 & & $\begin{array}{l}\text { Daphnia magna } \\
\text { Daphnia magna }\end{array}$ & $\begin{array}{l}48 \\
48\end{array}$ & \multirow{9}{*}{11.00} & & 1 \\
\hline Urea & $57-13-6$ & \multirow[t]{2}{*}{60.06} & -1.59 & \multirow{4}{*}{$\begin{array}{c}1.08 \mathrm{E}+06 \\
8.20 \mathrm{E}+05 \\
\text { miscible } \\
\text { miscible }\end{array}$} & Daphnia magna & 24 & & \multirow{2}{*}{ 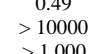 } & 3 \\
\hline 1,3,5-Triazine-2,4,6(1H,3H,5H)-trione, 1,3,5-tris(2-hydroxyethyl)- & $839-90-7$ & & -1.63 & & Daphnia magna & 48 & & & 3 \\
\hline Glyoxal & $107-22-2$ & \multirow{2}{*}{$\begin{array}{c}58.04 \\
270.13\end{array}$} & -1.65 & & Daphnia magna & 48 & & 404.00 & 1 \\
\hline 1,2,4-Butanetricarboxylic acid, 2-phosphono- & $37971-36-1$ & & -1.66 & & Daphnia magna & 24 & & \multirow{2}{*}{265.00} & 1 \\
\hline 1,2-Ethanediamine, N-[3-(trimethoxysilyl)propyl]- & 1760-24-3 & \multirow[t]{3}{*}{222.00} & -1.67 & $1.00 \mathrm{E}-06$ & Daphnia magna & $\begin{array}{r}48 \\
48\end{array}$ & & & 2 \\
\hline 4,4'-Diaminostilbene-2,2'-disulfonic acid & $81-11-8$ & & -1.70 & $3.20 \mathrm{E}+01$ & Daphnia magna & $\begin{array}{l}48 \\
24\end{array}$ & & $\begin{array}{c}90.00 \\
37.00 \\
210.00\end{array}$ & 2 \\
\hline Diazenedicarboxamide & $123-77-3$ & & -1.70 & $3.54 \mathrm{E}+04$ & Daphnia magna & 48 & & & 1 \\
\hline Citric acid & $77-92-9$ & 192.12 & -1.72 & $5.76 \mathrm{E}+05-7.71 \mathrm{E}+05$ & $\begin{array}{l}\text { Daphnia magna } \\
\text { Daphnia magna }\end{array}$ & $\begin{array}{l}48 \\
48\end{array}$ & & $\begin{array}{c}1535.00 \\
85.00\end{array}$ & 1 \\
\hline 13-Tridecanol, 2,5,8,11-tetraoxa- & $23783-42-8$ & 208.26 & -1.73 & $1.00 \mathrm{E}+06$ & & & & & 4 \\
\hline Triethylene glycol & $112-27-6$ & 150.18 & -1.75 & completely soluble & Daphnia magna & 48 & & 35.00 & 1 \\
\hline Glycerol & $56-81-5$ & 92.00 & -1.76 & $\begin{array}{l}\text { miscible } \\
\text { mats }\end{array}$ & Daphnia magna & 48 & & $>10000$ & 3 \\
\hline D-Gluconic acid & $526-95-4$ & 196.16 & -1.87 & $1.00 \mathrm{E}+03$ & Daphnia magna & 48 & & $>1000$ & 3 \\
\hline D-Glucono-1,5-lactone & $90-80-2$ & 178.14 & -1.98 & $5.90 \mathrm{E}+05$ & $\begin{array}{l}\text { Daphnia magna } \\
\text { Daphnia manga }\end{array}$ & $\begin{array}{l}24 \\
48\end{array}$ & & $\begin{array}{l}> \\
>\end{array} 1000$ & 3 \\
\hline Dipentaerythritol & $126-58-9$ & 254.00 & -2.00 & $3.00 \mathrm{E}+03$ & $\begin{array}{l}\text { Dappnia magna } \\
\text { Daphnia magna }\end{array}$ & $\begin{array}{l}48 \\
24\end{array}$ & & 100.00 & 1 \\
\hline Tetraethylene glycol & $112-60-7$ & 194.23 & -2.02 & miscible & Daphnia magna & 48 & & 7746.00 & 1 \\
\hline Ethylenediamine & $107-15-3$ & 60.10 & -2.04 & $1.10 \mathrm{E}+05$ & Daphnia magna & $\begin{array}{l}48 \\
48\end{array}$ & 17.00 & 2650 & 1 \\
\hline L-Ascorbic acid & $50-81-7$ & 176.12 & -2.05 & $3.30 \mathrm{E}+05$ & & & & 26.50 & 4 \\
\hline Stannane, dichlorodimethyl- & $753-73-1$ & 219.69 & -2.18 & $8.23 \mathrm{E}+02$ & Daphnia magna & 48 & 17.00 & & 1 \\
\hline 2,2'-Iminodiethanol & $111-42-2$ & 105.14 & -2.18 & miscible & Daphnia magna & 48 & & $\begin{array}{l}171.00 \\
10900\end{array}$ & 1 \\
\hline 2-Imidazolidinone, 4,5-dihydroxy-1,3-bis & $1854-26-8$ & 178.14 & -2.20 & & $\begin{array}{l}\text { Daphnia magna } \\
\text { Daphnia magna }\end{array}$ & $\begin{array}{l}48 \\
48\end{array}$ & & $>500$ & 3 \\
\hline D-Glucose & $50-99-7$ & 180.16 & -2.20 & & & & & & 4 \\
\hline Sulfuric acid & 7664-93-9 & 98.07 & -2.21 & miscible & Daphnia magna & 24 & & 29.00 & 1 \\
\hline Ethanol, 2-amino- & $141-43-5$ & 61.08 & -2.30 & & Daphnia magna & 48 & 32.60 & & 1 \\
\hline Pentaethylene glycol & $4792-15-8$ & 238.28 & -2.30 & $1.00 \mathrm{E}+06$ & Daphnia magna & 48 & & $>20,000$ & 3 \\
\hline Trimethyl methylamine hydroxide & $75-59-2$ & 91.15 & -2.47 & $1.00 \mathrm{E}+06$ & Daphnia magna & 48 & & 14.00 & 1 \\
\hline Morpholine & $110-91-8$ & 87.12 & -2.55 & miscible & Daphnia magna & 48 & & 45.00 & 1 \\
\hline Maleic anhydride & $108-31-6$ & 98.06 & -2.61 & $4.00 \mathrm{E}+05$ & Daphnia magna & 48 & & 33.00 & 1 \\
\hline D-Glucitol & $50-70-4$ & 182.17 & -3.10 & & & & & & 4 \\
\hline Tetraethylenepentamine & $112-57-2$ & 189.30 & -3.16 & $1.00 \mathrm{E}+06$ & Daphnia magna & 48 & & 24.10 & 1 \\
\hline Methanesulfinic acid, aminoimino- & $1758-73-2$ & 108.12 & -3.23 & $2.70 \mathrm{E}+04$ & $\begin{array}{l}\text { Daphnia magna } \\
\text { Daphnia magna }\end{array}$ & $\begin{array}{l}48 \\
24\end{array}$ & & $\begin{array}{l}14.60 \\
390.00\end{array}$ & 1 \\
\hline $\begin{array}{l}\text { Syrups, hydrolyzed starch } \\
\text { S }\end{array}$ & $8029-43-4$ & 180.16 & -3.24 & & & & & & 4 \\
\hline Glycine, N,N'-1,3-propanediylbis[N-(carboxymethyl)- & $1939-36-2$ & 306.27 & -3.37 & & & & & & 4 \\
\hline & & & & & Daphnia magna & 48 & & 297.00 & \\
\hline Phosphonic acid, [nitrilotris(methylene)]tris- & 6419-19-8 & 299.07 & -3.53 & $6.10 \mathrm{E}+05$ & Daphnia magna & 48 & & $\begin{array}{c}>25 \text { and } \\
<50\end{array}$ & 1 \\
\hline Glutamic acid & $\begin{array}{l}56-86-0 \\
57-50-1\end{array}$ & $\begin{array}{l}147.13 \\
342.30\end{array}$ & $\begin{array}{l}-3.69 \\
-3.70\end{array}$ & $8.64 \mathrm{E}+03$ & Daphnia magna & 48 & $>83.14$ & & 3 \\
\hline Sucrose & & 342.30 & $\begin{array}{r}-3.70 \\
-15\end{array}$ & $6675+05$ & Daphnia magna & 48 & & 36000.00 & 4 \\
\hline Methenamine & $100-97-0$ & 140.19 & -4.15 & $6.67 \mathrm{E}+05$ & Daphnia pulex & 48 & & 5.80 & 1 \\
\hline E.D.T.A & $60-00-4$ & 292.30 & -5.01 & $4.00 \mathrm{E}+02$ & & & & & 4 \\
\hline Phosphonic acid, [[(phosphonomethyl)imino]bis[2,1- & $15827-60-8$ & 573.20 & -9.72 & $5.00 \mathrm{E}+05$ & Daphnia magna & 48 & & 242.00 & 1 \\
\hline $\begin{array}{l}\text { ethanediylnitrilobis(methylene)]]tetrakis- } \\
\text { Maltodextrin }\end{array}$ & 9050-36-6 & 342.30 & & & Daphnia magna & 48 & & 667.00 & 4 \\
\hline Hexafluorosilicic acid & $16961-83-4$ & 144.09 & & & & & & & $\begin{array}{l}4 \\
4\end{array}$ \\
\hline
\end{tabular}


Table S7. Summary of reported toxicity test results using fish

\begin{tabular}{|c|c|c|c|c|c|c|c|c|c|c|}
\hline Chemicals name & CAS Reg. no & $\begin{array}{c}\text { Molecular } \\
\text { weight } \\
\left(\mathrm{g} \mathrm{mol}^{-1}\right)\end{array}$ & $\log \mathrm{K}_{\mathrm{ow}}$ & Solubility $\left(\mathrm{mg} \mathrm{L}^{-1}\right)$ & Species & Exposure method & $\begin{array}{l}\text { Duration } \\
\text { (h) }\end{array}$ & $\begin{array}{c}\text { Measured } \\
\mathrm{LC}_{50} \\
\left(\mathrm{mg} \mathrm{L}^{-1}\right)\end{array}$ & $\begin{array}{c}\text { Nominal } \\
\mathrm{LC}_{50} \\
\left(\mathrm{mg} \mathrm{L}^{-1}\right)\end{array}$ & Classification \\
\hline \multirow[b]{2}{*}{ C.I. Pigment Green 7} & \multirow[b]{2}{*}{$1328-53-6$} & \multirow[b]{2}{*}{1127.19} & \multirow[b]{2}{*}{17.40} & \multirow[b]{2}{*}{$7.00 \mathrm{E}-18-2.00 \mathrm{E}-16$} & Lepomis macrochirus & static & 96 & \multirow{4}{*}{$>0.52$} & 752.40 & \multirow[b]{2}{*}{2} \\
\hline & & & & & $\begin{array}{l}\text { Oncorhynchus mykiss } \\
\text { Oryzias latipes }\end{array}$ & $\begin{array}{l}\text { static } \\
\text { static }\end{array}$ & $\begin{array}{l}96 \\
48\end{array}$ & & $\begin{array}{l}355.60 \\
250.00\end{array}$ & \\
\hline Stannane, tetraoctyl- & $3590-84-9$ & 571.61 & 17.20 & $1.30 \mathrm{E}-12$ & Brachydanio rerio & semi-static, WAF & 96 & & & 3 \\
\hline Di-n-octyltin bis(ethylhexylthioglycolate) & $15571-58-1$ & 751.80 & 15.35 & $<1.00 \mathrm{E}+00$ & $\begin{array}{l}\text { Brachydanio rerio } \\
\text { Brachydanio rerio }\end{array}$ & $\begin{array}{l}\text { semi-static } \\
\text { static }\end{array}$ & $\begin{array}{l}96 \\
96\end{array}$ & & $>24.8$ & 3 \\
\hline Diisooctyl 2,2'-[(dioctylstannylene)bis(thio)]diacetate & 26401-97-8 & 751.80 & 15.35 & $<1.00 \mathrm{E}+00$ & Brachydanio rerio & semi-static & 96 & & $>24.8$ & 3 \\
\hline Triisooctyl 2,2',2"-[(octylstannylidyne)tris(thio)]triacetate & $26401-86-5$ & 841.90 & 14.40 & $5.00 \mathrm{E}-01-2.70 \mathrm{E}+00$ & $\begin{array}{l}\text { Brachydanio rerio } \\
\text { Brachydanio rerio }\end{array}$ & $\begin{array}{l}\text { semi-static } \\
\text { static }\end{array}$ & $\begin{array}{l}96 \\
96\end{array}$ & $\begin{array}{l}>2.3 \\
2.30\end{array}$ & & 1 \\
\hline $\begin{array}{l}\text { 2-Ethylhexyl 10-ethyl-4-[[2-[(2-ethylhexyl)oxy]-2-oxoethy]]thio]-4- } \\
\text { octyl-7-oxo-8-oxa-3,5-dithia-4-stannatetradecanoate }\end{array}$ & $27107-89-7$ & 841.90 & 14.10 & $5.00 \mathrm{E}-01-2.70 \mathrm{E}+00$ & Brachydanio rerio & semi-static & 96 & $>2.3$ & & 3 \\
\hline $\begin{array}{l}\text { Octadecanamide, N,N'-1,2-ethanediylbis- } \\
\text { Ditridecyl phthalate }\end{array}$ & $\begin{array}{l}110-30-5 \\
119-06-2\end{array}$ & $\begin{array}{l}593.04 \\
530.84\end{array}$ & $\begin{array}{l}13.98 \\
13.45\end{array}$ & $\begin{array}{l}1.10 \mathrm{E}-10 \\
1.48 \mathrm{E}-09\end{array}$ & & & & & & $\begin{array}{l}4 \\
4\end{array}$ \\
\hline Octadecyl 3-(3,5-di-tert-butyl-4-hydroxyphenvl)propionate & & & 13,40 & $\begin{array}{l}1.48 \mathrm{E}-09 \\
2.85 \mathrm{E}-02\end{array}$ & Lepomis macrochirus & static & 96 & >solubility & & 4 \\
\hline Octadecy 1 3-(3,5-dil-tert-butyl-4-hydroxyphenyl)propionate & $2082-19-3$ & 530.88 & 13.40 & 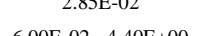 & Salmo gairdneri & static & 96 & >solubility & & 3 \\
\hline Monobutyltin tris (2-ethylhexylmercaptoacetate) & $26864-37-9$ & 785.79 & 12.45 & $6.00 \mathrm{E}-02-4.40 \mathrm{E}+00$ & Brachydanio rerio & semi-static & 96 & $>2.3$ & & 3 \\
\hline Bis-(2-Ethylhexyl) nonanedioate & $103-24-2$ & 412.66 & 11.90 & 4.00E-01 & Oryzias latipes & semi-static & 96 & & >solubility & 3 \\
\hline Di-Undecyl phthalate & $3648-20-2$ & 474.00 & 11.49 & $<1.70 \mathrm{E}-02$ & non toxic & & & & & 3 \\
\hline $\begin{array}{l}\text { 8-Oxa-3,5-dithia-4-stannatetradecanoic acid, 4,4-dibutyl-10-ethyl-7- } \\
\text { oxo-, 2-ethylhexyl ester }\end{array}$ & 10584-98-2 & 639.59 & 11.43 & $3.20 \mathrm{E}-01$ & $\begin{array}{l}\text { Brachydanio rerio } \\
\text { Brachydanio rerio }\end{array}$ & $\begin{array}{l}\text { static } \\
\text { static }\end{array}$ & $\begin{array}{l}96 \\
96\end{array}$ & $\begin{array}{l}>11 \\
12.00\end{array}$ & & 2 \\
\hline $\begin{array}{l}\text { 8-O-Ox-3,5-dithia-4-stannatetradecanoic acid, 10-ethyl-4-[[2-[(2-- } \\
\text { ethylhexyl)oxy]-2-oxoethyl]thio]-4-methyl-7-oxo-, 2-ethylhexyl } \\
\text { ester }\end{array}$ & 57583-34-3 & 743.71 & 10.98 & $1.80 \mathrm{E}+00-6.00 \mathrm{E}+00$ & non toxic & semi-static & & & & 3 \\
\hline Methacrylic acid, 2-methyl-, eicosyl ester & $45294-18-6$ & 366.63 & 10.61 & $5.01 \mathrm{E}-06$ & & & & & & 4 \\
\hline Di(isodecyl) phthalate & $26761-40-0$ & 446.68 & 10.36 & $1.04 \mathrm{E}-05$ & $\begin{array}{l}\text { Pimephales promelas } \\
\text { Lepomis macrochirus }\end{array}$ & $\begin{array}{l}\text { flow-through } \\
\text { static }\end{array}$ & $\begin{array}{l}96 \\
96\end{array}$ & $\begin{array}{l}\text { >solubility } \\
\text { >solubility }\end{array}$ & & 3 \\
\hline 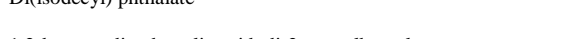 & & $4+40.00$ & & & Oncorhynchus mykiss & flow-through & 96 & solubility & & 3 \\
\hline 1,2-benzenedicarboxylic acid, di-2-propylheptyl ester & $53306-54-0$ & 446.68 & 10.36 & 2.24E-06 & non toxic & & & & & 3 \\
\hline 2-Butanone, $\mathrm{O}, \mathrm{O}^{\prime}, \mathrm{O}^{\prime}-($ ethenylsilylidyne)trioxime & 2224-33-1 & 313.48 & $\begin{array}{l}10.19 \\
0.91\end{array}$ & 3.00E-05 & & & & & & 4 \\
\hline Docosanoic acid & $112-85-6$ & 340.60 & 9.91 & $1.60 \mathrm{E}-02$ & Oryzias latipes & semi-static & 96 & & $>5.00$ & 3 \\
\hline 2-Butanone, $\mathrm{O}, \mathrm{O}^{\prime}, \mathrm{O}^{\prime}$-(methylsilylidyne)trioxime & 22984-54-9 & 301.46 & 9.83 & $6.00 \mathrm{E}-05$ & Oncorhynchus mykiss & static & 96 & & $>120$ & 3 \\
\hline 1-Docosanol & 661-19-8 & 326.61 & 9.68 & $2.70 \mathrm{E}-03$ & Oncorhynchus mykiss & semi-static & 96 & & $>1000$ & 3 \\
\hline Octadecyl methacrylate & $32360-05-7$ & 338.58 & 9.62 & $5.49 \mathrm{E}-05$ & & & & & & 4 \\
\hline Stannane, tetrabutyl- & $1461-25-2$ & 347.18 & 9.37 & $<1.00 \mathrm{E}-01$ & $\begin{array}{l}\text { Pimephales promelas } \\
\text { Pimenhales romelas }\end{array}$ & $\begin{array}{l}\text { flow-through } \\
\text { flow-through }\end{array}$ & $\begin{array}{l}96 \\
96\end{array}$ & 0.05 & 0.19 & 1 \\
\hline Di(isononyl) phthalate & 28553-12-0 & 418.62 & 9.37 & $2.32 \mathrm{E}-05$ & & & & & 0.19 & 4 \\
\hline Stannane, dioctyloxo- & $870-08-6$ & 361.16 & 9.26 & $6.57 \mathrm{E}-05$ & & & & & & 4 \\
\hline 1-Octadecene & $112-88-9$ & 252.49 & 9.04 & $1.51 \mathrm{E}-04$ & Salmo gairdneri & & 96 & & >solubility & 3 \\
\hline Cyclohexasiloxane, dodecamethyl- & $540-97-6$ & 444.39 & 8.82 & $5.13 \mathrm{E}-03$ & & & & & & 4 \\
\hline 1-Eicosanol & $629-96-9$ & 298.56 & 8.70 & $2.70 \mathrm{E}-03$ & & & & & & 4 \\
\hline Hexadecyl methacrylate & $2495-27-4$ & 310.52 & 8.64 & 4. $44 \mathrm{E}-04$ & & & & & & 4 \\
\hline Dimethylin bis[2-ethylhexylthioglycolate] & $57583-35-4$ & 555.42 & 8.48 & $1.00 \mathrm{E}-01-4.90 \mathrm{E}+00$ & Pimephales promelas & static & 96 & & >solubility & 3 \\
\hline & & & & & $\begin{array}{l}\text { Lepomis macrochirus } \\
\text { Pimphas }\end{array}$ & static & 96 & & >solubility & \\
\hline Bis(2-ethylhexyl) adipate & $103-23-1$ & 370.64 & 8.39 & $3.20 \mathrm{E}-03$ & $\begin{array}{l}\text { Pimephales promelas } \\
\text { Oncorhnychus mykiss }\end{array}$ & $\begin{array}{l}\text { static } \\
\text { static }\end{array}$ & $\begin{array}{l}96 \\
96\end{array}$ & & $\begin{array}{l}\text { >solubility } \\
\text { >solubility }\end{array}$ & 3 \\
\hline Terephthalic acid, bis(2-ethylhexyl) ester & $6422-86-2$ & 390.57 & 8.39 & $4.00 \mathrm{E}-04$ & $\begin{array}{l}\text { Oncorhynchus mykiss } \\
\text { Pimephales promelas }\end{array}$ & static & 96 & & $\begin{array}{c}>\text { solubility } \\
\geq 984\end{array}$ & 3 \\
\hline 1-Octadecanol & $112-92-5$ & 270.50 & 8.22 & $1.10 \mathrm{E}-03$ & Lenciscus idus & static & 96 & & >solubility & 3 \\
\hline Hexadecane & $544-76-3$ & 226.45 & 8.20 & $9.19 \mathrm{E}-04$ & non toxic & & & & & 3 \\
\hline Methacrylic acid, pentadecyl ester & $6140-74-5$ & 296.50 & 8.15 & $1.41 \mathrm{E}-03$ & & & & & & 4 \\
\hline Pigment Yellow 13 & 5102-83-0 & 685.62 & 8.11 & 3.50E-04 & & & & & & 4 \\
\hline 1-Hexadecen-3-ol, 3,7,11,15-tetramethyl- & $505-32-8$ & 296.54 & 8.10 & $5.80 \mathrm{E}+00$ & Leuciscus idus & static & 96 & & >solubility & 3 \\
\hline 1-Hexadecene & $629-73-2$ & 224.43 & 8.06 & $1.44 \mathrm{E}-03$ & $\begin{array}{l}\text { Oncorhynchus mykiss } \\
\text { Scopthalmus maximus }\end{array}$ & & $\begin{array}{l}96 \\
96\end{array}$ & & $\begin{array}{l}\text { > solubility } \\
\text { > solubility }\end{array}$ & 3 \\
\hline 1-Dodecene & $112-41-4$ & 168.33 & 8.00 & 1.13E-01 & Salmo gairdneri & static & 96 & 1.00 & & 2 \\
\hline Trisiloxane, 1,1,1,3,5,5,5-heptamethyl- & $1873-88-7$ & 222.51 & 7.84 & $2.00 \mathrm{E}-02$ & Brachydanio rerio & semi-static & 96 & $>0.108$ & & 3 \\
\hline $\begin{array}{l}\text { Trisiloxane, 1,1,1,5,5,5-hexamethyl-3,3-bis[(trimethylsilyl)oxy]- } \\
\text { (M4Q) }\end{array}$ & $3555-47-3$ & 384.85 & 7.80 & $1.50 \mathrm{E}-04$ & fish & flow-through & 96 & 0.000182 & & 3 \\
\hline $\begin{array}{l}\text { (M4Q) } \\
\text { Cyclodecane, 1,2,5,6,9,10-hexabromo- }\end{array}$ & $3194-55-6$ & 641.70 & 7.74 & 3.40E-03 & Oncorhynchus mykiss & & 96 & & $>=0.0025$ & 3 \\
\hline Pentadecane & $629-62-9$ & 212.42 & 7.71 & $2.87 \mathrm{E}-03$ & non toxic & & & & & 3 \\
\hline Tetradecyl methacrylate & $2549-53-3$ & 282.47 & 7.66 & 4.46E- 03 & & & & & & 4 \\
\hline
\end{tabular}


(Table S7. continued)

\begin{tabular}{|c|c|c|c|c|c|c|c|c|c|c|}
\hline Chemicals name & CAS Reg. no & $\begin{array}{c}\text { Molecular } \\
\text { weight } \\
\left(\mathrm{g} \mathrm{mol}^{-1}\right)\end{array}$ & $\log \mathrm{K}_{\mathrm{ow}}$ & Solubility $\left(\mathrm{mg} \mathrm{L}^{-1}\right)$ & Species & Exposure method & $\begin{array}{l}\text { Duration } \\
\text { (h) }\end{array}$ & $\begin{array}{l}\text { Measured } \\
\mathrm{LC}_{50} \\
\left(\mathrm{mg} \mathrm{L}^{-1}\right)\end{array}$ & $\begin{array}{l}\text { Nominal } \\
\mathrm{LC}_{50} \\
\left(\mathrm{mg} \mathrm{L}^{-1}\right)\end{array}$ & Classification \\
\hline Pentadecane & $\begin{array}{l}629-62-9 \\
2540-33\end{array}$ & 212.42 & 7.71 & $2.87 \mathrm{E}-03$ & non toxic & & & & & 3 \\
\hline Tetradecyl methacrylate & 2549-53-3 & 282.47 & 7.66 & $4.46 \mathrm{E}-03$ & & & & & & 4 \\
\hline Octadecanamine, N,N-dimethyl-, N-oxide & 2571-88-2 & 313.57 & 7.62 & $3.18 \mathrm{E}-03$ & Brachydanio rerio & semi-static & 96 & & 1.40 & 2 \\
\hline $\begin{array}{l}\text { Butanamide, 2,2'-[(3,3'-dichloro[1,1'-biphenyl]-4,4'- } \\
\text { diyl)bis(azo)]bis[N-(4-chloro-2,5-dimethoxyphenyl)-3-oxo- }\end{array}$ & $5567-15-7$ & 818.50 & 7.54 & $8.10 \mathrm{E}-03$ & Brachydanio rerio & static & 96 & & no effects & 3 \\
\hline $\begin{array}{l}\text { 9--Cctadecen-1-ol, (9Z)- } \\
\text { Phenol dodecyl. }\end{array}$ & $\begin{array}{c}143-28-2 \\
27193-86-8\end{array}$ & $\begin{array}{l}268.49 \\
262.44\end{array}$ & $\begin{array}{l}7.50 \\
7.46\end{array}$ & $4.20 \mathrm{E}-02$ & & & & & & $\begin{array}{l}4 \\
4\end{array}$ \\
\hline Phenol, dodecyl- & $27193-86-8$ & 262.44 & 7.46 & & & & & & & 4 \\
\hline Benzene, undecyl- & 6742-54-7 & 232.41 & 7.45 & $4.10 \mathrm{E}-02$ & $\begin{array}{l}\text { Salmo gairdneri } \\
\text { Pimphales promelas } \\
\text { Lepomis macrochirus }\end{array}$ & $\begin{array}{l}\text { static } \\
\text { static } \\
\text { static }\end{array}$ & $\begin{array}{l}96 \\
96 \\
96\end{array}$ & & $\begin{array}{l}\text { > water sol } \\
\text { > water sol } \\
\text { > water sol }\end{array}$ & 3 \\
\hline Tetradecane & $629-59-4$ & 198.40 & 7.20 & $9.19 \mathrm{E}-03$ & non toxic & static & & & $>$ water sol & 3 \\
\hline Tridecyl 2-methacrylate & $2495-25-2$ & 268.44 & 7.17 & & & & & & & 4 \\
\hline Phenol, tetrapropylene & $57427-55-1$ & & 7.14 & & & & & & & 4 \\
\hline 1-Tetradecene & $1120-36-1$ & 196.38 & 7.08 & $4.00 \mathrm{E}-04$ & Oncorhynchus mykiss & semi-static & 96 & & > water sol & 3 \\
\hline C.I. Pigment Yellow 12 & 6358-85-6 & 629.51 & 7.05 & $4.00 \mathrm{E}-04$ & Brachydanio rerio & static & 96 & & $\begin{array}{c}\text { >water } \\
\text { solubility }\end{array}$ & 3 \\
\hline Bis(2-ethylhexyl) phthalate & $117-81-7$ & 390.57 & 7.00 & $1.30 \mathrm{E}+00$ & $\begin{array}{l}\text { Pimephales promelas } \\
\text { Oryzias latipes } \\
\text { Oncorhynchus mykiss }\end{array}$ & & $\begin{array}{l}96 \\
96 \\
96\end{array}$ & & $\begin{array}{l}\text { >solubility } \\
\text { >solubility } \\
\text { >solubility }\end{array}$ & 3 \\
\hline Cyclotetrasiloxane, octamethyl- & $556-67-2$ & 296.62 & 6.79 & & & & & & & 4 \\
\hline Tridecane & $629-50-5$ & 184.37 & 6.73 & & & & & & & 4 \\
\hline 1-Hexadecanol & $\begin{array}{c}36653-82-4 \\
142-90-5\end{array}$ & $\begin{array}{r}242.45 \\
254.42\end{array}$ & $\begin{array}{l}6.73 \\
6.68\end{array}$ & $1.30 \mathrm{E}-02$ & Salmo gairdneri & semistatic & 96 & & $>0.4$ & 3 \\
\hline Dodecyl methacrylate & $142-90-5$ & 254.42 & 6.68 & & Brachydani rerio & semi-static & 9696 & & & 4 \\
\hline hexadecyldimethylamine $\mathrm{N}$-oxide & 7128-91-8 & 285.52 & 6.64 & $3.20 \mathrm{E}-02$ & $\begin{array}{l}\text { Brachydanolo rerio } \\
\text { Brachydanio rerio }\end{array}$ & 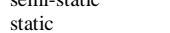 & 9090 & & $\begin{array}{l}0.45 \\
0.37\end{array}$ & 2 \\
\hline Trisiloxane, octamethyl- & $107-51-7$ & 236.54 & 6.60 & $3.40 \mathrm{E}-02$ & Oncorhynchus mykiss & & 96 & $>0.019$ & & 3 \\
\hline Methyl laurate & $111-82-0$ & 214.35 & 6.50 & $1.39 \mathrm{E}+00$ & Oryzias latipes & & 96 & $>0.52$ & & 3 \\
\hline Decanedioic acid, bis(2,2,6,6-tetramethyl-4-piperidinyl) ester & $52829-07-9$ & 480.74 & 6.50 & $1.88 \mathrm{E}+01$ & $\begin{array}{l}\text { Oncorhynchus mykiss } \\
\text { Lepomis macrochirus }\end{array}$ & & $\begin{array}{l}96 \\
96\end{array}$ & $\begin{array}{l}4.30 \\
4.30\end{array}$ & & 1 \\
\hline Benzene, 1,1'-oxybis-, octabromo deriv. & $32536-52-0$ & 801.38 & 6.29 & $5.00 \mathrm{E}-04$ & & & & & & 4 \\
\hline Benzene, 1,1'-oxybis[2,3,4,5,6-pentabromo]- & 1163-19-5 & 959.17 & 6.27 & $<1.00 \mathrm{E}-05$ & Oryzias latipes & & 48 & & $>$ solubility & 3 \\
\hline & & & & & Oryzias latipes & semistatic & 96 & & $>5.0$ & \\
\hline p-Cresol, 6,6'-di-tert-butyl-2,2'-methylenedi- & $119-47-1$ & 340.51 & 6.25 & $2.00 \mathrm{E}-02$ & Oryzias latipes & semistatic & 48 & & $>500$ & 2 \\
\hline 1-Pentadecanol & $629-76-5$ & 228.42 & 6.24 & $1.02 \mathrm{E}-01$ & Pimephales promelas & static & 96 & & 0.31 & 4 \\
\hline Dodecane & $112-40-3$ & 170.34 & 6.23 & $1.10 \mathrm{E}-01$ & & & & & & 4 \\
\hline 1-Dodecanethiol & $112-55-0$ & 202.40 & 6.20 & $1.00 \mathrm{E}+00$ & & & & & & 4 \\
\hline 1,1-(1,1-dimethyl-3-methylene-1,3-propanediyl)bisbenzene & $6362-80-7$ & 236.36 & 6.20 & $2.30 \mathrm{E}-01$ & Oryzias latipes & semi-static & & $>0.092$ & & 3 \\
\hline tert-Dodecanethiol & $25103-58-6$ & 202.40 & 6.20 & $2.50 \mathrm{E}-01$ & 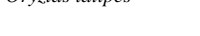 & serm sture & & - 0.072 & & 4 \\
\hline Cyclohexane, 1,1'-methylenebis[4-isocyanato- & $5124-30-1$ & 262.35 & 6.11 & $1.21 \mathrm{E}-01$ & Danio rerio & static & 96 & $>8.1$ & & 3 \\
\hline Phenol, 2,6-bis(1,1-dimethylethyl)-4-(1-methylpropyl)- & $17540-75-9$ & 262.44 & 6.10 & $2.50 \mathrm{E}+00$ & & & & & & 4 \\
\hline Dodecene & 25378-22-7 & 168.33 & 6.10 & $1.25 \mathrm{E}-01$ & & & & & & 4 \\
\hline Ethanol, 2,2'-(octadecyloxidoimino)bis- & $14048-77-2$ & 373.63 & 6.08 & $2.90 \mathrm{E}-02$ & & & & & & 4 \\
\hline Phenol, nonyl- & 25154-52-3 & 220.36 & 5.99 & $1.57 \mathrm{E}+00$ & $\begin{array}{l}\text { Pimephales promelas } \\
\text { Lepomis macrochirus }\end{array}$ & $\begin{array}{l}\text { low-through } \\
\text { flow-through }\end{array}$ & 96 & 0.13 & & 1 \\
\hline 1,2,4-Benzenetricarboxylic acid, tris(2-ethylhexyl) ester & $3319-31-1$ & 546.79 & 5.94 & $1.30 \mathrm{E}-01$ & Oryzias latipes & $\begin{array}{l}\text { flow-through } \\
\text { flons }\end{array}$ & 96 & & $>100$ & 3 \\
\hline 2,2',6,6'-Tetrabromo-4,4'-isopropylidenediphenol & 79-94-7 & 543.90 & 5.90 & $1.26 \mathrm{E}+00$ & Pimephales promelas & & 96 & 0.54 & & 1 \\
\hline Ethanol, 2,2'-[(9Z)-9-octadecenyloxidoimino]bis- & 93962-62-0 & 371.61 & 5.86 & $4.50 \mathrm{E}-02$ & Brachydanio rerio & semi-static & 96 & 0.70 & & 2 \\
\hline Triisobutylene & 7756-94-7 & 168.33 & 5.85 & $1.90 \mathrm{E}-01$ & & & & & & 4 \\
\hline Stannane, dichlorodiocytyl- & $3542-36-7$ & 416.07 & 5.82 & $2.40 \mathrm{E}-01-2.80 \mathrm{E}-01$ & $\begin{array}{l}\text { Brachydanio rerio } \\
\text { STlmogivdnori }\end{array}$ & semi-static & ${ }_{24}^{96}$ & $>0.24$ & & 3 \\
\hline Benzene, dodecyl- & $123-01-3$ & 246.44 & 5.75 & $4.10 \mathrm{E}-02$ & $\begin{array}{l}\text { Salmo gairdneri } \\
\text { Pimephales promelas }\end{array}$ & $\begin{array}{l}\text { static } \\
\text { static }\end{array}$ & $\begin{array}{l}24-96 \\
24-96\end{array}$ & $\begin{array}{l}\text { >solubility } \\
\text { >solubility }\end{array}$ & & 3 \\
\hline & & & & & Lepomis macrochi & static & $24-96$ & >solubility & & \\
\hline 1-Tetradecanol & $112-72-1$ & 214.39 & 5.75 & $1.91 \mathrm{E}-01$ & non toxic & & & & & 3 \\
\hline $\mathrm{n}$-Undecane & $1120-21-4$ & 156.31 & 5.74 & $4.00 \mathrm{E}-03$ & Oryzias latipes & semi-static & 96 & $>0.013$ & & 3 \\
\hline Decene & $25339-53-1$ & 140.27 & 5.70 & $2.10 \mathrm{E}-01$ & & & & & & 4 \\
\hline Phosphine, triphenyl- & 603-35-0 & 262.29 & 5.69 & $1.65 \mathrm{E}-01$ & Leuciscus idus & static & 96 & & $>10000$ & 3 \\
\hline 1-Tetradecanamine, $\mathrm{N}, \mathrm{N}$-dimethyl-, $\mathrm{N}$-oxide & $3332-27-2$ & 257.46 & 5.66 & 3.20E-01 & Brachydanio rerio & semi-static & 96 & 2.40 & & 2 \\
\hline Nonane & $111-84-2$ & 128.26 & 5.65 & $4.06 \mathrm{E}-01$ & & & & & & 4 \\
\hline
\end{tabular}


(Table S7. continued)

\begin{tabular}{|c|c|c|c|c|c|c|c|c|c|c|}
\hline Chemicals name & CAS Reg. no & $\begin{array}{c}\text { Molecular } \\
\text { weight } \\
\left(\mathrm{g} \mathrm{mol}^{-1}\right)\end{array}$ & $\log \mathrm{K}_{\mathrm{ow}}$ & Solubility $\left(\mathrm{mg} \mathrm{L}^{-1}\right)$ & Species & Exposure method & $\begin{array}{l}\text { Duration } \\
\text { (h) }\end{array}$ & $\begin{array}{c}\text { Measured } \\
\mathrm{LC}_{50} \\
\left(\mathrm{mg} \mathrm{L}^{-1}\right)\end{array}$ & $\begin{array}{c}\text { Nominal } \\
\mathrm{LC}_{50} \\
\left(\mathrm{mg} \mathrm{L}^{-1}\right)\end{array}$ & Classification \\
\hline Peroxide, bis(1-methyl-1-phenylethyl)- & $80-43-3$ & 270.37 & 5.60 & $4.30 \mathrm{E}-01$ & & & & & & 4 \\
\hline Bumetrizole & $3896-11-5$ & 315.81 & 5.55 & $<1.00 \mathrm{E}+00$ & Danio rerio & static & 96 & $\begin{array}{l}>\text { water } \\
\text { solubility }\end{array}$ & & 3 \\
\hline Metilox & $6386-38-5$ & 292.42 & $5.50-5.90$ & $2.20 \mathrm{E}+00$ & Brachydanio rerio & flow-through & 96 & & $\begin{array}{l}\text { >water } \\
\text { solubility }\end{array}$ & 3 \\
\hline 2-Ethylhexyl vinyl ether & $103-44-6$ & 156.27 & 5.50 & $1.80 \mathrm{E}+00$ & & & & & & 4 \\
\hline $\begin{array}{l}\text { Peroxide, [1,3(or 1,4)-phenylenebis(1-methylethylidene)]bis[(1,1- } \\
\text { dimethylethyl) }\end{array}$ & $25155-25-3$ & 338.49 & 5.50 & $4.00 \mathrm{E}-02$ & Poecilia reticulata & semi-static & 96 & $\begin{array}{l}\text { >water } \\
\text { solubility }\end{array}$ & & 3 \\
\hline 1-Dodecanamine, N,N-dimethyl- & $112-18-5$ & 213.41 & 5.47 & $1.00 \mathrm{E}+01$ & $\begin{array}{l}\text { Brachydanio rerio } \\
\text { Leuciscus idus }\end{array}$ & & $\begin{array}{l}96 \\
48\end{array}$ & & 0.55 & 1 \\
\hline $\begin{array}{l}\text { 1,3,5-Triazine-2,4,6(1H,3H,5H)-trione, 1,3,5-tris [[3,5-bis(1,1- } \\
\text { dimethylethyl)-4-hydroxyphenyl] methyl]- }\end{array}$ & $27676-62-6$ & 784.10 & 5.45 & $1.00 \mathrm{E}+01$ & & & & & & 4 \\
\hline $\begin{array}{l}\text { Ethanone, 1-(5,6,7,8-tetrahydro-3,5,5,6,8,8-hexamethyl-2- } \\
\text { naphthalenyl)- }\end{array}$ & $1506-02-1$ & 258.41 & 5.40 & $1.22 \mathrm{E}+00$ & Lepomis macrochirus & flow-through & 96 & 1.49 & & 2 \\
\hline $\begin{array}{l}\text { Ethanone, 1-(5,6,7,8-tetrahydro-3,5,5,6,8,8-hexamethyl-2- } \\
\text { naphthalenyl)- }\end{array}$ & $21145-77-7$ & 258.41 & 5.40 & $1.22 \mathrm{E}+00$ & Lepomis macrochirus & flow-through & 96 & 1.49 & & 2 \\
\hline $\begin{array}{l}\text { Benzene, 1,4-dimethyl-2-(1-phenylethyl) } \\
2,2 \text {, } 3,3 \text {-Tetrachloro-4,4'-diaminodiphenylmethane (TCDAM) }\end{array}$ & $6165-51-1$ & 210.31 & $\begin{array}{l}5.39 \\
5.39\end{array}$ & $\begin{array}{l}9.60 \mathrm{E}-01 \\
207 \mathrm{E}\end{array}$ & Orizias latipes & semi-static & 96 & & 0.31 & 1 \\
\hline $\begin{array}{l}2,2^{2}, 3,33^{\prime} \text {-Tetrachloro-4,4'-diaminodiphenylmethane (TCDAM) } \\
\text { Disiloxane, 1,3-diethenyl-1,1,3,3-tetramethyl- }\end{array}$ & $\begin{array}{c}42240-73-3 \\
2627-95-4\end{array}$ & $\begin{array}{l}322.02 \\
186.40\end{array}$ & $\begin{array}{l}5.39 \\
5.36\end{array}$ & $\begin{array}{l}2.27 \mathrm{E}-01-7.88 \mathrm{E}-01 \\
\quad 2.07 \mathrm{E}-01\end{array}$ & Oncorhynchus mykiss & flow-through & 96 & $>0.13$ & >solubility & $\begin{array}{l}4 \\
3\end{array}$ \\
\hline Stannane, dibutyloxo- & $818-08-6$ & 248.94 & 5.33 & $6.73 \mathrm{E}-01$ & Brachydanio rerio & static & 96 & $>3$ & & 3 \\
\hline $\begin{array}{l}\text { Cyclopenta[g]-2-benzopyran, 1,3,4,6,7,8-hexahydro-4,6,6,7,8,8- } \\
\text { hexamethyl- }\end{array}$ & $1222-05-5$ & 258.41 & 5.30 & $1.75 \mathrm{E}+00$ & & & & & & 4 \\
\hline Isodecyl methacrylate & 29964-84-9 & 226.36 & 5.28 & & & & & & & 4 \\
\hline $\begin{array}{l}\text { 1(3H)-Isobenzofuranone, 6-(dimethylamino)-3,3-bis[4- } \\
\text { (dimethylamino)phenyl]- }\end{array}$ & $1552-42-7$ & 415.54 & 5.27 & $1.00 \mathrm{E}+01$ & & & & & & 4 \\
\hline 1 -Tridecanol & $112-70-9$ & 200.37 & 5.26 & $3.80 \mathrm{E}-01$ & non toxic & & & & & 3 \\
\hline Triphenylmethyl chloride & $76-83-5$ & 278.78 & 5.25 & $5.35 \mathrm{E}-01$ & & & & & & 4 \\
\hline n-Decane & $124-18-5$ & 142.29 & 5.25 & & & & & & & 4 \\
\hline $\begin{array}{l}\text { Diphenylmethane diisocyanate (4,4'-M.D.I.) } \\
2^{2} \text { '-2inhenyl methane dijscacrante }\end{array}$ & $\begin{array}{l}101-68-8 \\
2536-05-2\end{array}$ & $\begin{array}{r}250.25 \\
25026\end{array}$ & 5.22 & & non toxic & & & & & 3 \\
\hline $\begin{array}{l}2,2-\text { Diphenyl methanae ensisocyanate } \\
\text { 2,4-Diphenyl methane disocyanate }\end{array}$ & $5873-54-1$ & 250.26 & 5.22 & & & & & & & $\begin{array}{l}4 \\
4\end{array}$ \\
\hline Benzene, 1,1 '-methylenebis(isocyanato- & $26447-40-5$ & 250.26 & 5.22 & $8.29 \mathrm{E}-01$ & non toxic & & & & & 3 \\
\hline Disiloxane, hexamethyl- & $107-46-0$ & 162.38 & 5.20 & $9.30 \mathrm{E}-01$ & Oncorhynchus mykiss & flow-through & 96 & 0.46 & & 1 \\
\hline 1-Isotridecanol & 27458-92-0 & 200.37 & 5.19 & $5.24 \mathrm{E}+00$ & fish & & 96 & $0.42-11$ & & 1 \\
\hline Octane & 111-65-9 & 114.23 & 5.18 & $1.15 \mathrm{E}+00$ & & & & & & 4 \\
\hline Ligroine & $8032-32-4$ & 114.23 & 5.18 & & & & & & & 4 \\
\hline 1-Decanol & $112-30-1$ & 158.29 & 5.13 & $3.95 \mathrm{E}+01$ & Pimephales promelas & flow-through & 96 & 2.30 & & 2 \\
\hline 1-Dodecanol & $112-53-8$ & 186.34 & 5.13 & $1.90 \mathrm{E}+00$ & Pimphales promelas & flow-through & 96 & 1.01 & & 1 \\
\hline 1-Decene & $872-05-9$ & 140.27 & 5.12 & $1.15 \mathrm{E}-01$ & Salmo gairdneri & static & 96 & & $>1,000$ & 3 \\
\hline 2,6-Di-tert-butyl-p-cresol & $128-37-0$ & 220.36 & 5.10 & $1.10 \mathrm{E}+00$ & $\begin{array}{l}\text { Oryzias latipes } \\
\text { Brachydanio rerio }\end{array}$ & $\begin{array}{l}\text { semi-static } \\
\text { static }\end{array}$ & $\begin{array}{l}48 \\
96\end{array}$ & & $\begin{array}{l}5.00 \\
>\text { solubility }\end{array}$ & 2 \\
\hline $\begin{array}{l}\text { 2-Propenoic acid, 2-methyl-, (1R,2R,4R)-1,7,7- } \\
\text { trimethylbicyclo[2.2.1]hept-2-yl ester, rel- }\end{array}$ & 7534-94-3 & 222.33 & 5.09 & $5.44 \mathrm{E}+00$ & Danio rerio & & 96 & 1.79 & & 1 \\
\hline Silane, dichlorodiphenyl- & $80-10-4$ & 253.20 & 5.06 & $2.80 \mathrm{E}+00$ & & & & & & 4 \\
\hline Cyclopentadiene, hexachloro- & $77-47-4$ & 272.77 & 5.04 & $1.03 \mathrm{E}+00-1.25 \mathrm{E}+00$ & $\begin{array}{l}\text { Pimephales promelas } \\
\text { Pimenhales romelas }\end{array}$ & $\begin{array}{l}\text { flow-through } \\
\text { static }\end{array}$ & $\begin{array}{l}96 \\
96\end{array}$ & 0.01 & 0.18 & 1 \\
\hline Pentaerythritol tetrakis[3-(3,5-di-tert-butyl-4-hydroxypheny & $6683-19-8$ & 1177.67 & 5.00 & $1.00 \mathrm{E}-01$ & Non toxic & & & & & 3 \\
\hline Pentene, 2,4,4-trimethyl- & $25167-70-8$ & 112.22 & 5.00 & $1.80 \mathrm{E}+00$ & Oncorhynchus mykiss & semi-static & 96 & 0.58 & & 1 \\
\hline 2-Ethylhexyl methacrylate & 688-84-6 & 198.31 & $4.95-5.59$ & $1.60 \mathrm{E}+00$ & Oryzias latipes & static & 96 & 2.78 & & 2 \\
\hline 2-Benzothiazolesulfenamide, N-cyclohexyl- & 95-33-0 & 264.41 & 4.93 & $3.20 \mathrm{E}-01$ & Oryzias latipes & flow-through & 96 & 2.10 & & 2 \\
\hline 5-tert-Butyl-2,4,6-trinitro-m-xylene (Musk xylene) & $81-15-2$ & 297.26 & 4.90 & $1.50 \mathrm{E}-01$ & Lepomis macrochirus & static & 96 & & 1.20 & 2 \\
\hline Neodecanoic acid, ethenyl ester & $51000-52-3$ & 198.30 & 4.90 & $5.90 \mathrm{E}+00$ & & & & & & 4 \\
\hline Butyl benzyl phthalate & 85-68-7 & 312.36 & 4.84 & & & & & & & 4 \\
\hline Perfluorooctanoic Acid & $335-67-1$ & 414.07 & 4.81 & $9.50 \mathrm{E}+03$ & Oncorhynchus mykiss & & 96 & & 707.00 & 1 \\
\hline 2-Benzothiazolesulfenamide, N,N-dicyclohexyl- & $4979-32-2$ & 346.55 & 4.80 & $1.90 \mathrm{E}-03$ & $\begin{array}{l}\text { Orizias latipes } \\
\text { Orizias latipes }\end{array}$ & $\begin{array}{l}\text { semi-static } \\
\text { semi-static }\end{array}$ & $\begin{array}{l}96 \\
96\end{array}$ & & $\begin{array}{l}>1000 \\
>0.0334\end{array}$ & 3 \\
\hline Benzenesulfonic acid, dodecyl- & $27176-87-0$ & 326.50 & 4.78 & $6.03 \mathrm{E}-01$ & $\begin{array}{l}\text { Salmo gairdnei } \\
\text { Pimephales promelas }\end{array}$ & & $\begin{array}{l}96 \\
96\end{array}$ & & $\begin{array}{l}3.20-5.60 \\
0.26\end{array}$ & 2 \\
\hline Phenol, 5-chloro-2-(2,4-dichlorophenoxy)- & $3380-34-5$ & 289.54 & 4.76 & $1.00 \mathrm{E}+01$ & $\begin{array}{l}\text { Lepomis macrochirus } \\
\text { Brachydanio rerio }\end{array}$ & & $\begin{array}{l}96 \\
96\end{array}$ & & $\begin{array}{l}0.37 \\
0.54\end{array}$ & 1 \\
\hline
\end{tabular}




\section{(Table S7. continued)}

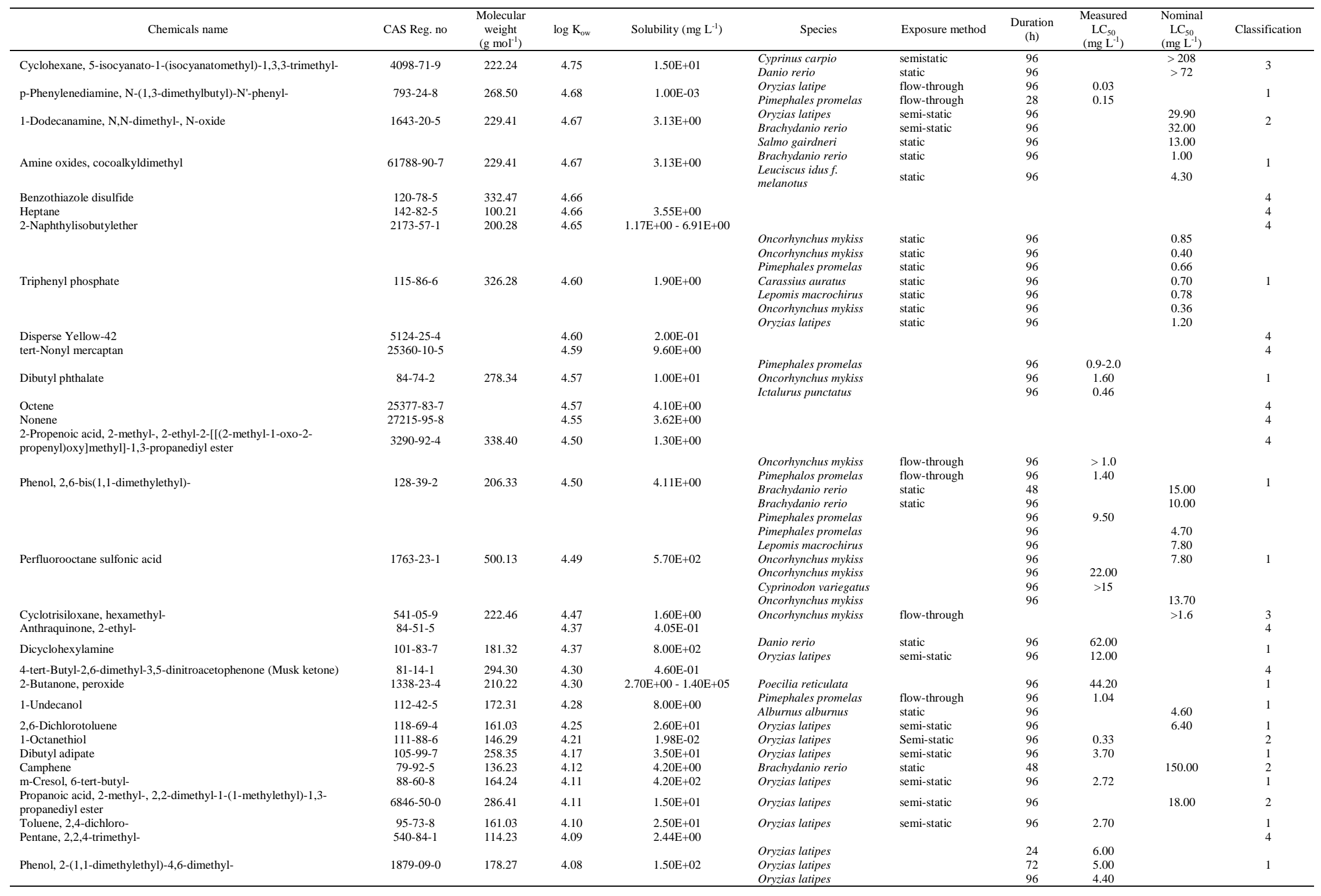


(Table S7. continued)

\begin{tabular}{|c|c|c|c|c|c|c|c|c|c|c|}
\hline Chemicals name & CAS Reg. no & $\begin{array}{c}\text { Molecular } \\
\text { weight } \\
\left(\mathrm{g} \mathrm{mol}^{-1}\right)\end{array}$ & $\log \mathrm{K}_{\mathrm{ow}}$ & Solubility $\left(\mathrm{mg} \mathrm{L}^{-1}\right)$ & Species & Exposure method & $\begin{array}{c}\text { Duration } \\
\text { (h) }\end{array}$ & $\begin{array}{c}\text { Measured } \\
\text { LC }_{5_{0}} \\
\left(\mathrm{mg} \mathrm{L}^{-1}\right) \\
\end{array}$ & $\begin{array}{c}\text { Nominal } \\
L_{50} \\
\left(\mathrm{mg} \mathrm{L}^{-1}\right) \\
\end{array}$ & Classification \\
\hline Benzene, 1,4-diethyl- & $105-05-5$ & 134.22 & 4.06 & $1.70 \mathrm{E}+01$ & Oryzias latipes & & 96 & & 1.80 & 1 \\
\hline Benzene, 1,2,4-trichloro- & $120-82-1$ & 181.45 & 4.02 & $3.60 \mathrm{E}+01-4.80 \mathrm{E}+01$ & $\begin{array}{l}\text { Lepomis macrochirus } \\
\text { Pimephales promelas }\end{array}$ & $\begin{array}{l}\text { flow through } \\
\text { flow through }\end{array}$ & $\begin{array}{l}96 \\
96\end{array}$ & $\begin{array}{l}3.02 \\
3.01\end{array}$ & & 1 \\
\hline 1,1'-Biphenyl & $92-52-4$ & 154.21 & 4.01 & $7.48 \mathrm{E}+00$ & Oncorhynchus mykiss & static & 96 & 1.50 & & 1 \\
\hline 3-Buten-2-one, 4-(2,6,6-trimethyl-1-cyclohexen-1-yl)- & $79-77-6$ & 192.30 & 4.00 & $1.69 \mathrm{E}+02$ & Pimephales promelas & flow-through & 96 & 5.10 & & 1 \\
\hline 3,5,9-Undecatrien-2-one, 6,10-dimethyl- & $141-10-6$ & 192.30 & 4.00 & $9.70 \mathrm{E}+01$ & $\begin{array}{l}\text { Leuciscus idus } \\
\text { Oncorhynchus mykiss }\end{array}$ & $\begin{array}{l}\text { static } \\
\text { static }\end{array}$ & $\begin{array}{l}96 \\
48\end{array}$ & $\begin{array}{l}6.80 \\
7.10\end{array}$ & & 1 \\
\hline Heptene & $25339-56-4$ & & 3.99 & $1.82 \mathrm{E}+01$ & 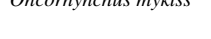 & 保 & & & & 4 \\
\hline Isooctyl acrylate & $29590-42-9$ & 184.20 & 3.93 & $1.24 \mathrm{E}+01$ & Pimephales promelas & & 96 & 0.67 & & 1 \\
\hline Acenaphthylene, 1,2-dihydro- & $83-32-9$ & & 3.92 & $3.80 \mathrm{E}-03$ & & & & & & 4 \\
\hline Sulfone, bis(p-chlorophenyl) & $80-07-9$ & 287.16 & 3.90 & $8.60 \mathrm{E}-01$ & Brachydanio rerio & static-renewal & 24 & & $>10$ & 3 \\
\hline \multirow[t]{2}{*}{ 2-Benzothiazolesulfeamide, N-(1,1-dimethylethyl)- } & $95-31-8$ & 238.37 & 3.90 & $3.45 \mathrm{E}-01$ & Oryzias latipes & semi-static & 96 & 0.35 & & 1 \\
\hline & $101-72-4$ & 226.32 & 3.90 & $1.50 \mathrm{E}+01$ & $\begin{array}{l}\text { Lepomis macrochirus } \\
\text { Oncorhynchus mykiss }\end{array}$ & $\begin{array}{l}\text { static } \\
\text { static }\end{array}$ & $\begin{array}{l}96 \\
96\end{array}$ & & $\begin{array}{l}0.43 \\
0.34\end{array}$ & 1 \\
\hline p-Phenylenediamine, $\mathrm{N}$-(1-methylethyl)-N'-phenyl- & & & & & Pimephales promelas & static & 96 & 0.41 & & \\
\hline 2-Ethylhexyl acrylate & $103-11-7$ & 184.28 & 3.90 & $9.60 \mathrm{E}+00$ & Oncorhynchus mykiss & & 96 & 1.80 & & 1 \\
\hline \multirow[b]{2}{*}{ Linalylacetate } & $110-54-3$ & 86.18 & 3.90 & & & & & & & 4 \\
\hline & $115-95-7$ & 196.29 & 3.90 & $3.00 \mathrm{E}+01$ & $\begin{array}{l}\text { Cyprinus carpio } \\
\text { Oryzias latipes }\end{array}$ & $\begin{array}{l}\text { flow-through } \\
\text { semi-static }\end{array}$ & $\begin{array}{l}96 \\
96\end{array}$ & $\begin{array}{c}11.00 \\
1.50\end{array}$ & & 1 \\
\hline Phenol, 2,4,6-tribromo- & $118-79-6$ & 330.80 & 3.89 & $5.90 \mathrm{E}+01$ & $\begin{array}{l}\text { Orzzias latipes } \\
\text { Cyprinus carpio } \\
\text { Pimephales promelas }\end{array}$ & $\begin{array}{l}\text { semi-static } \\
\text { static } \\
\text { flow-through }\end{array}$ & $\begin{array}{l}96 \\
96 \\
96\end{array}$ & $\begin{array}{c}1.50 \\
1.10 \\
6.5-6.8\end{array}$ & & 1 \\
\hline Naphthalene, methyl- & $1321-94-4$ & 142.20 & 3.87 & & & & & & & 4 \\
\hline Naphthalene, 2-methyl- & $91-57-6$ & 142.20 & 3.86 & $2.46 \mathrm{E}+01$ & Oncorhynchus mykiss & & 96 & 1.46 & & 1 \\
\hline 1H-Indene, 3a,4,7,7a-tetrahydro- & $3048-65-5$ & 120.19 & 3.83 & $3.74 \mathrm{E}+01$ & Oryzias latipes & semi-static & 96 & 4.40 & & 1 \\
\hline \multirow[t]{2}{*}{ 2-Norbornene, 5-ethylidene- } & $16219-75-3$ & 120.19 & 3.82 & $8.00 \mathrm{E}+01$ & $\begin{array}{l}\text { Oryzias latipes } \\
\text { Brachydanio rerio }\end{array}$ & $\begin{array}{l}\text { semi-static } \\
\text { semi-static }\end{array}$ & $\begin{array}{l}96 \\
96\end{array}$ & $\begin{array}{l}7.00 \\
7.60\end{array}$ & & 1 \\
\hline & & & & & Oncorhynchus mykiss & & 96 & & 16.00 & \\
\hline Aniline, 4-nitro-N-phenyl- & $836-30-6$ & 214.00 & 3.82 & $4.10 \mathrm{E}+01$ & 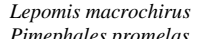 & flow-through & 96 & & 17.00 & 1 \\
\hline Silane, dichloromethylphenyl- & $149-74-6$ & & 3.80 & $7.85 \mathrm{E}+01$ & Pimephales promelas & flow-through & 24 & 2.20 & & 4 \\
\hline Naphthalene, 1,2,3,4-tetrahydro- & $119-64-2$ & 132.21 & 3.78 & $4.50 \mathrm{E}+01$ & Danio rerio & semi-static & 96 & 3.20 & & 1 \\
\hline 1-Pentanamine, N-pentyl- & 2050-92-2 & 157.30 & 3.76 & $4.44 \mathrm{E}+02$ & Oncorhynchus mykiss & & 96 & 3.90 & & 1 \\
\hline Anthraquinone, 1-amino- & $82-45-1$ & & 3.74 & $3.20 \mathrm{E}+01$ & Oryzias latipes & semi-static & 96 & & >solubility & 3 \\
\hline 2-Ethylhexyl acetate & $103-09-3$ & 172.27 & 3.74 & $3.90 \mathrm{E}+00$ & Oncorhynchus mykiss & static-renewal & 96 & 8.27 & & 2 \\
\hline Naphthalene & 91-20-3 & 128.18 & 3.73 & $3.00 \mathrm{E}+01$ & Oncorhynchus mykiss & & 96 & 1.46 & & 1 \\
\hline Isodecanol & 25339-17-7 & 158.29 & 3.71 & $2.00 \mathrm{E}+00-2.40 \mathrm{E}+02$ & & & & & & 4 \\
\hline 1-Heptanol, 2-propyl- & $10042-59-8$ & 158.29 & 3.71 & & & & & & & 4 \\
\hline Diphenyl tolyl phosphate & $26444-49-5$ & & 3.70 & $2.40 \mathrm{E}+00$ & Oryzias latipes & & 96 & 1.30 & & 1 \\
\hline Phenol, 4-(1,1,3,3-tetramethylbutyl)- & $140-66-9$ & 206.33 & 3.70 & $1.90 \mathrm{E}+01$ & Pimephales promelas & flow-through & 96 & 0.29 & & 1 \\
\hline Silane, triethoxyoctyl- & 2943-75-1 & & 3.70 & $1.30 \mathrm{E}-01$ & Oncorhynchus mykiss & flow-through & 96 & $>0.055$ & & 3 \\
\hline Decanamine, N,N-dimethyl-, N-oxide & $2605-79-0$ & 201.36 & 3.69 & $3.04 \mathrm{E}+01$ & & & . & & & 4 \\
\hline Amines, C10-16-alkyldimethyl, N-oxides & $70592-80-2$ & 201.36 & 3.69 & $3.04 \mathrm{E}+01$ & Lepomis macrochirus & static & 96 & & 3.18 & 1 \\
\hline Isooctyl mercaptoacetate & 25103-09-7 & 20100 & $3.68-3.96$ & $1.06 \mathrm{E}+01$ & Pimephales promelas & static & 96 & & 4.40 & 1 \\
\hline \multirow{5}{*}{$\begin{array}{l}\text { N-(2-metnybuty) I-1-pentanamine } \\
\text { 4,4'-Methylenebis-(2-chlorobenzenamine)* }\end{array}$} & $61361-18-0$ & 157.30 & 3.68 & (1) & - & 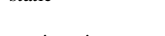 & (5) & & (1) & 4 \\
\hline & 101-14-4 & & 3.66 & $5.09 \mathrm{E}-01$ & Oryzias latipes & semi-static & 96 & & $0.61-0.66$ & 1 \\
\hline & & & & & Cyprinodon variegatus & & 96 & 4.70 & & \\
\hline & & & & & Oncorhynchus mykiss & & 96 & 4.80 & & \\
\hline & & & & & Poecilia reticulata & & 96 & 5.10 & & \\
\hline \multirow[t]{3}{*}{ Cumene } & $98-82-8$ & 120.19 & 3.66 & $7.50 \mathrm{E}+01$ & Salmo gairdneri & & $\begin{array}{l}96 \\
48\end{array}$ & $\begin{array}{r}2.70 \\
22.50\end{array}$ & & 1 \\
\hline & & & & & $\begin{array}{l}\text { Leuciscus saus } \\
\text { Leuciscus idus }\end{array}$ & & 48 & 47.00 & & \\
\hline & & & & & $\begin{array}{l}\text { melanotus } \\
\text { Pimehale promelas }\end{array}$ & & $\begin{array}{l}48 \\
96\end{array}$ & 20.00 & & \\
\hline Cyclopropanecarboxylic acid, 3-(2,2-dichloroethenyl)-2,2-dimethyl-, & 61898-95-1 & & 3.66 & $5.30 \mathrm{E}+01$ & Oncorhynchus mykiss & semi-static & 96 & 3.01 & & 1 \\
\hline \multirow{2}{*}{ Benzene, 1,2,4-trimethyl- } & 95-63-6 & & 3.63 & & & & & & & 4 \\
\hline & & & & & Oncorhynchus mykiss & & 24 & 2.30 & & \\
\hline \multirow{2}{*}{ Pyridine, 2,3,5,6-tetrachloro- } & 2402-79-1 & 216.87 & 3.63 & $2.94 \mathrm{E}+01-3.02 \mathrm{E}+01$ & Oncorhynchus mykiss & & 48 & 1.50 & & 1 \\
\hline & & & & & $\begin{array}{l}\text { Oncorhynchus mykiss } \\
\text { Oncorhnnchus mykiss }\end{array}$ & & $\begin{array}{l}72 \\
96\end{array}$ & $\begin{array}{l}1.50 \\
1.50\end{array}$ & & \\
\hline
\end{tabular}


(Table S7. continued)

\begin{tabular}{|c|c|c|c|c|c|c|c|c|c|c|}
\hline Chemicals name & CAS Reg. no & $\begin{array}{c}\text { Molecular } \\
\text { weight } \\
\left(\mathrm{g} \mathrm{mol}^{-1}\right)\end{array}$ & $\log \mathrm{K}_{\mathrm{ow}}$ & Solubility (mg L ${ }^{-1}$ ) & Species & Exposure method & $\begin{array}{l}\text { Duration } \\
\text { (h) }\end{array}$ & $\begin{array}{c}\text { Measured } \\
\mathrm{LC}_{50} \\
\left(\mathrm{mg} \mathrm{L}^{-1}\right)\end{array}$ & $\begin{array}{c}\text { Nominal } \\
\mathrm{LC}_{50} \\
\left(\mathrm{mg} \mathrm{L}^{-1}\right)\end{array}$ & Classification \\
\hline \multirow{2}{*}{$\begin{array}{l}\text { 2-Methyl-N-(2-methylbutyl)-1-butanamine } \\
\text { Silane, trichlorophenyl- }\end{array}$} & $\begin{array}{l}27094-65-1 \\
98-13-5\end{array}$ & 157.30 & 3.61 & $785 \mathrm{~F}+01$ & & & & & & 4 \\
\hline & $98-13-5$ & & 3.60 & $7.85 \mathrm{E}+01$ & $\begin{array}{l}\text { Oncorhynchus mykiss } \\
\text { Oryzias latipes }\end{array}$ & $\begin{array}{l}\text { static } \\
\text { semi-static }\end{array}$ & 96 & 028 & $>100$ & 3 \\
\hline Phenol, 2-sec-butyl-4,6-dinitro- & $88-85-7$ & 240.21 & 3.57 & $3.45 \mathrm{E}+01$ & $\begin{array}{l}\text { Pimephales promelas } \\
\text { Poecilia reticulatas }\end{array}$ & $\begin{array}{l}\text { flow-through } \\
\text { semi-static }\end{array}$ & 96 & 0.54 & & 1 \\
\hline 1-Octene & $111-66-0$ & 112.21 & $3.50-4.60$ & $4.10 \mathrm{E}+00$ & Salmo gairdneri & $\begin{array}{l}\text { Semilstatic } \\
\text { static }\end{array}$ & 96 & 0.11 & 100.00 & 1 \\
\hline 2,6-Naphthalenedicarboxylic acid, dimethyl ester & $840-65-3$ & & 3.50 & $1.50 \mathrm{E}-01$ & $\begin{array}{l}\text { Dryzias latipes } \\
\text { Orio }\end{array}$ & $\begin{array}{l}\text { Static } \\
\text { semi-static }\end{array}$ & $\begin{array}{l}96 \\
48\end{array}$ & $>0.1$ & 4.80 & 3 \\
\hline \multirow[t]{3}{*}{ Formic acid, chloro-, 2-ethylhexyl ester } & 24468-13-1 & 192.69 & 3.50 & $4.90 \mathrm{E}+01$ & $\begin{array}{l}\text { Leuciscus idus } \\
\text { Danio rerio }\end{array}$ & $\begin{array}{l}\text { static } \\
\text { static }\end{array}$ & $\begin{array}{l}96 \\
96\end{array}$ & & $\begin{array}{l}3.16 \\
4.18\end{array}$ & 1 \\
\hline & & & & & Oncorhynchus mykiss & static & 96 & 11.00 & & \\
\hline & & & & & Oncorhynchus mykiss & flow-through & 96 & 13.00 & & \\
\hline \multirow[t]{2}{*}{ Tributyl phosphate } & $126-73-8$ & 266.31 & 3.50 & $4.00 \mathrm{E}+02$ & Brachydanio rerio & static & 96 & $\sim 11.8$ & & 1 \\
\hline & & & & & Leuciscus idus & static & 96 & 7.60 & & \\
\hline Phenol 2 - ec buttl- & & & & & Pimephales promelas & static & 96 & 6.40 & & \\
\hline $\begin{array}{l}\text { Phenol, 2-sec-butyl- } \\
\text { Beprenesulfonyl chloride 4-methyl- }\end{array}$ & $89-72-5$ & 150.22 & 3.49 & $1.52 \mathrm{E}+03$ & Oryzias latipes & semi-static & 96 & & 6.00 & 1 \\
\hline $\begin{array}{l}\text { Benzenesulfonyl chloride, 4-methyl- } \\
\text { Benzene, (1-methylethenyl)- }\end{array}$ & $\begin{array}{r}98-59-9 \\
98-83-9\end{array}$ & 190.65 & 3.49 & $5.12 \mathrm{E}+01$ & $\begin{array}{l}\text { Oryzias latipes } \\
\text { Orzigas latives }\end{array}$ & $\begin{array}{l}\text { static } \\
\text { semi-static }\end{array}$ & 96 & 1500 & 55.00 & 2 \\
\hline \multirow{2}{*}{$\begin{array}{l}\text { Propanoic acid, 2-methyl-, monoester with 2,2,4-trimethyl-1,3- } \\
\text { pentanediol }\end{array}$} & $25265-77-4$ & 21632 & 3.47 & $858 \mathrm{E}+02$ & Pimephales promelas & static & 96 & 15.00 & 30.00 & 1 \\
\hline & & $210.02-5$ & & & Brachydanio rerio & flow-through & 96 & 2.10 & & \\
\hline \multirow{2}{*}{ Benzene, 1,4-dichloro- } & $106-46-7$ & 147.00 & 3.44 & $6.00 \mathrm{E}+01-7.00 \mathrm{E}+01$ & Pimephales promelas & static & 96 & & 14.20 & 1 \\
\hline & & & & & $\begin{array}{l}\text { Pimephales promelas } \\
\text { Oncorhynchus mykiss }\end{array}$ & $\begin{array}{l}\text { flow-through } \\
\text { flow-through }\end{array}$ & $\begin{array}{l}96 \\
96\end{array}$ & $\begin{array}{l}4.20 \\
1.12\end{array}$ & & 1 \\
\hline Cyclohexane & $110-82-7$ & 84.16 & 3.44 & $5.80 \mathrm{E}+01$ & Pimephales promelas & flow-through & 96 & 4.53 & & 1 \\
\hline Benzoyl peroxide & $94-36-0$ & 242.24 & 3.43 & $9.10 \mathrm{E}+00$ & Oryzias latipes & flow-through & 96 & 0.24 & $000-1$ & 1 \\
\hline \multirow{2}{*}{ Methacrylic acid, 3-(trichlorosilyl)propyl ester } & 7351-61-3 & & 3.43 & $9.87 \mathrm{E}+01$ & Poecilia reticulata & semi-static & 96 & & 2.00 & 4 \\
\hline & & & & & Oncorhynchus mykiss & flow-through & 96 & 2.30 & & \\
\hline Toluene, 2-chloro- & $95-49-8$ & 126.58 & 3.42 & $4.70 \mathrm{E}+01$ & Alburnus alburnus & & 96 & & $6.7-9.1$ & 1 \\
\hline \multirow{2}{*}{$\begin{array}{l}\text { Benzene, 1,3,5-trimethyl- } \\
\text { 1-Hexanol, 3,5,5-trimethyl- }\end{array}$} & $108-67-8$ & 120.20 & 3.42 & $4.00 \mathrm{E}+01-7.50 \mathrm{E}+01$ & Oryzias latipes & & & & & 4 \\
\hline & $3452-97-9$ & & 3.42 & $4.50 \mathrm{E}+02$ & Oryzias latipes & semi-static & 96 & 27.70 & & 1 \\
\hline \multirow{2}{*}{ 2-Hydroxy-3-napthoic acid } & $92-70-6$ & 188.18 & $3.40-3.59$ & $4.74 \mathrm{E}+02$ & Oryzias latipes & semi-static & 96 & $>82.2$ & & 1 \\
\hline & & & & & $\begin{array}{l}\text { Brachydanio rerio } \\
\text { Brutaps }\end{array}$ & static & 96 & $\begin{array}{c}68.00 \\
0.00\end{array}$ & & \\
\hline \multirow[t]{3}{*}{ Bisphenol A } & $80-05-7$ & 228.29 & 3.40 & $3.00 \mathrm{E}+02$ & $\begin{array}{l}\text { Or. latpes } \\
\text { Or. latipes }\end{array}$ & & 72 & 6.80 & & 1 \\
\hline & & & & & Or. latipes & & 72 & 5.10 & 520 & \\
\hline & & & & & Brachydanio rerio & flow-through & 96 & & 5.20 & \\
\hline \multirow[t]{2}{*}{ Benzene, 1,2-dichloro- } & $95-50-1$ & 147.00 & 3.40 & $1.56 \mathrm{E}+02$ & Salmo gairdneri & flow-through & 96 & 1.61 & & 1 \\
\hline & $1490-04-6$ & & 340 & $508 \mathrm{~F}+02$ & Lepomis macrochirus & static & 96 & & 5.60 & 4 \\
\hline DL-menthol & $1+90-0+0$ & & 0.40 & $5.08 \mathrm{E}+02$ & Pimephales promelas & flow though & 96 & 18.40 & & \\
\hline \multirow[t]{2}{*}{ L-Menthol } & $2216-51-5$ & 156.27 & 3.40 & $4.31 \mathrm{E}+02$ & Danio rerio & & 96 & 15.60 & & 1 \\
\hline & 15356602 & 15627 & 340 & $420 \mathrm{~F}+02-500 \mathrm{~F}+02$ & Oryzias latipes & semi-static & 48 & 26.00 & & 4 \\
\hline (+)-Menthol & $13530-60-2$ & 156.27 & 3.40 & $4.20 \mathrm{E}+02-5.00 \mathrm{E}+02$ & Jordanella floridae & flow-through & 96 & 8.40 & & 4 \\
\hline Ethene, tetrachloro- & $127-18-4$ & 165.83 & 3.40 & $1.60 \mathrm{E}+02$ & Pimephales promelas & flow-through & 96 & 18.40 & & 1 \\
\hline Hexene & $25264-93-1$ & & 339 & $5.00 \mathrm{E}+01$ & Oncorhynchus mykiss & flow-through & 96 & 5.00 & & 4 \\
\hline 1-Hexene & $592-41-6$ & 84.16 & 3.39 & $5.00 \mathrm{E}+01$ & $\begin{array}{l}\text { Oncorhynchus mykiss } \\
\text { Brachiodanio rerio }\end{array}$ & $\begin{array}{l}\text { Semi-static } \\
\text { Semi-static }\end{array}$ & $\begin{array}{l}96 \\
96\end{array}$ & & $\begin{array}{c}5.60 \\
25.00\end{array}$ & 1 \\
\hline \multirow{2}{*}{$\begin{array}{l}\text { Pentane } \\
\text { Dibutyl maleate }\end{array}$} & $109-66-0$ & & 3.39 & $\begin{array}{l}3.85 \mathrm{E}+01 \\
1.7 \mathrm{E}+02\end{array}$ & $\begin{array}{l}\text { Brachodanio rerio } \\
\text { Oncorhynchus myiss }\end{array}$ & $\begin{array}{l}\text { Sem-1static } \\
\text { semistatic } \\
\text { semi-statc }\end{array}$ & 96 & 4.26 & & 1 \\
\hline & $105-76-0$ & & 3.38 & $1.73 \mathrm{E}+02$ & $\begin{array}{l}\text { oncorhynchus mykiss } \\
\text { Oryzias latipes }\end{array}$ & $\begin{array}{l}\text { semi-static } \\
\text { semi-static }\end{array}$ & $\begin{array}{l}96 \\
48\end{array}$ & 0.90 & 31.00 & 1 \\
\hline Butane, 1,1'-oxybis- & $142-96-1$ & 130.23 & 3.35 & $1.13 \mathrm{E}+02$ & Pimephales promelas & flow-through & 96 & 32.30 & 31.00 & 1 \\
\hline Neodecanoyl chloride & $40292-82-8$ & 190.72 & 3.35 & $6.77 \mathrm{E}+01$ & $\begin{array}{l}\text { Brachydanio rerio } \\
\text { Oncorhynchus mykiss }\end{array}$ & $\begin{array}{l}\text { static } \\
\text { semi-static }\end{array}$ & $\begin{array}{l}96 \\
96\end{array}$ & & $\begin{array}{l}138.00 \\
37.20\end{array}$ & 1 \\
\hline Toluene, 4-chloro- & $106-43-4$ & 126.59 & 3.33 & $4.00 \mathrm{E}+01$ & $\begin{array}{l}\text { Oryzias latipe } \\
\text { Brachydanio rerio }\end{array}$ & $\begin{array}{l}\text { semi-static } \\
\text { static }\end{array}$ & $\begin{array}{l}48 \\
96\end{array}$ & & $\begin{array}{r}5.20 \\
2400\end{array}$ & 1 \\
\hline
\end{tabular}


(Table S7. continued)

\begin{tabular}{|c|c|c|c|c|c|c|c|c|c|c|}
\hline Chemicals name & CAS Reg. no & $\begin{array}{c}\text { Molecular } \\
\text { weight } \\
\left(\mathrm{g} \mathrm{mol}^{-1}\right) \\
\end{array}$ & $\log \mathrm{K}_{\mathrm{ow}}$ & Solubility $\left(\mathrm{mg} \mathrm{L}^{-1}\right)$ & Species & Exposure method & $\begin{array}{l}\text { Duration } \\
\text { (h) }\end{array}$ & $\begin{array}{c}\text { Measured } \\
\mathrm{LC}_{50} \\
\left(\mathrm{mg} \mathrm{L}^{-1}\right) \\
\end{array}$ & $\begin{array}{c}\text { Nominal } \\
\text { LC } \\
\left(\mathrm{mg} \mathrm{L}^{-1}\right) \\
\end{array}$ & Classification \\
\hline Benzene, 1-chloro-2-(chloromethyl)- & $611-19-8$ & 161.03 & 3.32 & $1.00 \mathrm{E}+02$ & $\begin{array}{l}\text { Oryzias latipes } \\
\text { Danio rerio } \\
\text { Pimephales promelas }\end{array}$ & $\begin{array}{l}\text { flow-through } \\
\text { static } \\
\text { static }\end{array}$ & $\begin{array}{l}96 \\
96 \\
96\end{array}$ & $\begin{array}{c}0.27 \\
0.5-0.71 \\
0.71-0.96\end{array}$ & & 1 \\
\hline Thymol & $89-83-8$ & & 3.30 & & & & & & & 4 \\
\hline Phenol, 4-tert-butyl- & $98-54-4$ & 150.22 & 3.29 & $6.10 \mathrm{E}+02$ & $\begin{array}{l}\text { Oryzias latipes } \\
\text { Pimephales promelas }\end{array}$ & $\begin{array}{l}\text { semi-static } \\
\text { semi-static }\end{array}$ & $\begin{array}{l}96 \\
96\end{array}$ & $\begin{array}{l}5.10 \\
5.10\end{array}$ & & 1 \\
\hline 2,6-di-tert-Butyl-4-ethylphenol & $4130-42-1$ & & 3.27 & $2.10 \mathrm{E}+01$ & & & & & & 4 \\
\hline Cyclohexanamine, 4,4'-methylenebis- & $1761-71-3$ & 210.37 & 3.26 & $6.42 \mathrm{E}+02$ & $\begin{array}{l}\text { Leuciscus idus } \\
\text { Leuciscus idus }\end{array}$ & & $\begin{array}{l}96 \\
96\end{array}$ & & $\begin{array}{l}67.80 \\
>100\end{array}$ & 2 \\
\hline Phenol, 2,4-dichloro- & $120-83-2$ & 163.00 & 3.25 & $4.50 \mathrm{E}+03$ & $\begin{array}{l}\text { Pimephales promelas } \\
\text { Platichtys flesus } \\
\text { Oryzias latipes }\end{array}$ & $\begin{array}{l}\text { flow-through } \\
\text { semi-static } \\
\text { static }\end{array}$ & $\begin{array}{l}96 \\
96 \\
48\end{array}$ & $\begin{array}{c}7.75-11.6 \\
5.99 \\
8.60\end{array}$ & & 1 \\
\hline $\begin{array}{l}\text { Silane, (3-chloropropyl)trichloro- } \\
\text { Silane, tetramethyl- }\end{array}$ & $\begin{array}{l}2550-06-3 \\
75-76-3\end{array}$ & & $\begin{array}{l}3.24 \\
3.24\end{array}$ & $\begin{array}{l}6.44 \mathrm{E}+01 \\
1.96 \mathrm{E}+01\end{array}$ & Oncorhynchus mykiss & flow-through & 96 & 1.90 & & $\begin{array}{l}4 \\
1\end{array}$ \\
\hline Diallyl phthalate & $131-17-9$ & 246.26 & 3.23 & $1.48 \mathrm{E}+02$ & $\begin{array}{l}\text { Oncorhynchus mykiss } \\
\text { Orizias latipes }\end{array}$ & $\begin{array}{l}\text { semi-static } \\
\text { semi-static }\end{array}$ & $\begin{array}{l}96 \\
96\end{array}$ & $\begin{array}{l}0.23 \\
0.44\end{array}$ & & 1 \\
\hline $\begin{array}{l}\text { Isononanol } \\
\text { Diphenyl carbonate } \\
\text { Pentane, 2-methyl- } \\
\text { Hexamethylene diisocyanate }\end{array}$ & $\begin{array}{c}27458-94-2 \\
102-09-0 \\
107-83-5 \\
822-06-0\end{array}$ & $\begin{array}{l}144.26 \\
214.22 \\
86.18 \\
168.20\end{array}$ & $\begin{array}{l}3.22 \\
3.21-3.28 \\
3.21 \\
3.20\end{array}$ & $1.30 \mathrm{E}+01$ & Danio rerio & static & 96 & 3.90 & & $\begin{array}{l}4 \\
1 \\
4 \\
4\end{array}$ \\
\hline 1,3-Xylene & $108-38-3$ & 106.17 & 3.20 & $1.61 \mathrm{E}+02$ & $\begin{array}{l}\text { Oncorhynchus mykiss } \\
\text { Pimephales promelas }\end{array}$ & & $\begin{array}{l}96 \\
96\end{array}$ & 8.40 & 28.00 & 1 \\
\hline Peroxide, bis(tert-butyl)- & $110-05-4$ & & 3.20 & $1.71 \mathrm{E}+02$ & Poecilia reticulate & semi-static & 96 & & $\begin{array}{l}\text { >water } \\
\text { solubility }\end{array}$ & 3 \\
\hline Benzene, 1,2-dichloro-3-nitro- & $3209-22-1$ & 192.00 & 3.20 & $7.41 \mathrm{E}+01$ & Oryzias latipes & semi-static & 48 & 8.60 & & 1 \\
\hline 1,4-Xylene & $106-42-3$ & 106.17 & 3.15 & $1.62 \mathrm{E}+02$ & $\begin{array}{l}\text { Oncorhynchus mykiss } \\
\text { Pimephales promelas } \\
\text { Menidia menidia }\end{array}$ & flow-through & $\begin{array}{l}96 \\
96 \\
96\end{array}$ & 2.60 & 26.70 & 1 \\
\hline Benzene, ethyl- & $100-41-4$ & 106.20 & 3.15 & $1.52 \mathrm{E}+02$ & $\begin{array}{l}\text { Pimephales promelas } \\
\text { Oncorhynchus mykiss }\end{array}$ & $\begin{array}{l}\text { flow-through } \\
\text { static }\end{array}$ & $\begin{array}{l}96 \\
96\end{array}$ & $\begin{array}{l}5.10 \\
12.10\end{array}$ & 4.20 & 1 \\
\hline $\begin{array}{l}\text { Ethanol, 2,2'-(dodecyloxidoimino)bis- } \\
\text { Silane, (3-chloropropyl)triethoxy- }\end{array}$ & $\begin{array}{l}2530-44-1 \\
5089-70-3\end{array}$ & 289.46 & $\begin{array}{l}3.13 \\
3.13\end{array}$ & $\begin{array}{l}2.99 \mathrm{E}+01 \\
1.13 \mathrm{E}+02\end{array}$ & $\begin{array}{l}\text { Brachydanio rerio } \\
\text { Brachydanio rerio }\end{array}$ & $\begin{array}{l}\text { semi-static } \\
\text { semi-static }\end{array}$ & $\begin{array}{l}96 \\
96\end{array}$ & 80.00 & 7.90 & $\begin{array}{l}1 \\
1\end{array}$ \\
\hline o-Xylene & $95-47-6$ & 106.17 & 3.12 & $1.78 \mathrm{E}+02$ & $\begin{array}{l}\text { Oncorhynchus mykiss } \\
\text { Pimephales promelas }\end{array}$ & & $\begin{array}{l}96 \\
96\end{array}$ & $\begin{array}{c}7.60 \\
16.40\end{array}$ & & 1 \\
\hline $\begin{array}{l}\text { Stannane, dibutylbis[(1-oxododecyl)oxy]- } \\
\text { Propane, 1-(ethenyloxy)-2-methyl- }\end{array}$ & $\begin{array}{l}77-58-7 \\
109-53-5\end{array}$ & & $\begin{array}{l}3.12 \\
3.10\end{array}$ & $\begin{array}{l}7.00 \mathrm{E}-02 \\
7.20 \mathrm{E}+02\end{array}$ & $\begin{array}{l}\text { Brachydanio rerio } \\
\text { Danio rerio }\end{array}$ & $\begin{array}{l}\text { static } \\
\text { semi-static }\end{array}$ & $\begin{array}{l}96 \\
96\end{array}$ & 28.30 & $>2.6$ & $\begin{array}{l}3 \\
1\end{array}$ \\
\hline & & & & & Lepomis macrochirus & & $\begin{array}{l}96 \\
24\end{array}$ & & $\begin{array}{l}2.30 \\
3.80\end{array}$ & \\
\hline Phenol, 4-chloro-2-methyl- & $1570-64-5$ & 142.59 & 3.09 & $2.30 \mathrm{E}+03$ & $\begin{array}{l}\text { Oryzias latipes } \\
\text { Brachydanio rerio } \\
\text { Salmo trutta }\end{array}$ & & $\begin{array}{l}96 \\
96 \\
24\end{array}$ & & $\begin{array}{l}6.80 \\
6.30 \\
4.50\end{array}$ & 1 \\
\hline Hexamethylene diacrylate & 13048-33-4 & 226.27 & 3.08 & & samo truta & & & & & 4 \\
\hline Dodecanedioic acid & 693-23-2 & 230.30 & 3.07 & $3.00 \mathrm{E}+01$ & $\begin{array}{l}\text { Leuciscus idus } \\
\text { Oryzias latipes }\end{array}$ & static & $\begin{array}{l}48 \\
48\end{array}$ & & $\begin{array}{l}3.10 \\
7.01\end{array}$ & 4 \\
\hline 4-Nitrobenzene, 1,2-dichloro- & 99-54-7 & 192.00 & 3.04 & $1.21 \mathrm{E}+02$ & $\begin{array}{l}\text { Leuciscus idus } \\
\text { melanotus }\end{array}$ & static & 48 & & 8.00 & 1 \\
\hline & & & & & $\begin{array}{l}\text { Leuciscus idus } \\
\text { Leuciscus idus }\end{array}$ & $\begin{array}{l}\text { static } \\
\text { static }\end{array}$ & $\begin{array}{l}48 \\
96\end{array}$ & & $\begin{array}{l}2.00 \\
10.00\end{array}$ & \\
\hline Butyl methacrylate & $97-88-1$ & 142.20 & 3.03 & $3.60 \mathrm{E}+02$ & $\begin{array}{l}\text { Oryzias latipes } \\
\text { Pimenhale sromelas }\end{array}$ & $\begin{array}{l}\text { stal-static } \\
\text { semi-stic } \\
\text { flow-through }\end{array}$ & $\begin{array}{l}96 \\
96\end{array}$ & 5.57 & & 1 \\
\hline $\begin{array}{l}\text { 1,3-Butadiene, 2,3-dichloro- } \\
\text { Benzene, (trifluoromethyl)- }\end{array}$ & $\begin{array}{c}1653-19-6 \\
98-08-8\end{array}$ & 122.98 & $\begin{array}{l}3.02 \\
3.01\end{array}$ & $1.92 \mathrm{E}+02$ & $\begin{array}{l}\text { Pimephales promelas } \\
\text { Danio rerio } \\
\text { Oryzias latipes }\end{array}$ & $\begin{array}{l}\text { flow-through } \\
\text { semi-static }\end{array}$ & $\begin{array}{l}96 \\
96\end{array}$ & 19.00 & 10.00 & $\begin{array}{l}1 \\
1\end{array}$ \\
\hline Dibutyltin, maleate- & $78-04-6$ & 346.99 & 3.01 & & $\begin{array}{l}\text { Brachydanio rerio } \\
\text { Orvzias latipes }\end{array}$ & & $\begin{array}{l}96 \\
96\end{array}$ & $\begin{array}{l}>5.7 \\
13.12\end{array}$ & & 1 \\
\hline $\begin{array}{l}\text { 1-Octanol } \\
\text { Cyclopentane }\end{array}$ & $\begin{array}{l}111-87-5 \\
287-92-3\end{array}$ & $\begin{array}{r}130.23 \\
70.14\end{array}$ & $\begin{array}{l}3.00 \\
3.00\end{array}$ & $\begin{array}{l}5.51 \mathrm{E}+02 \\
1.56 \mathrm{E}+02\end{array}$ & $\begin{array}{l}\text { P. promelas } \\
\text { Pros }\end{array}$ & flow-through & 96 & $\begin{array}{l}13.12 \\
13.00\end{array}$ & & $\begin{array}{l}1 \\
4\end{array}$ \\
\hline Citral & $5392-40-5$ & 152.23 & 3.00 & $5.90 \mathrm{E}+02$ & $\begin{array}{l}\text { Leuciscus idus } \\
\text { Oryzias latipes }\end{array}$ & $\begin{array}{l}\text { static } \\
\text { semi-static }\end{array}$ & $\begin{array}{l}96 \\
96\end{array}$ & & $\begin{array}{c}4.6-10 \\
4.10\end{array}$ & 1 \\
\hline
\end{tabular}


(Table S7. continued)

\begin{tabular}{|c|c|c|c|c|c|c|c|c|c|c|}
\hline Chemicals name & CAS Reg. no & $\begin{array}{c}\text { Molecular } \\
\text { weight } \\
\left(\mathrm{g} \mathrm{mol}^{-1}\right)\end{array}$ & $\log \mathrm{K}_{\mathrm{ow}}$ & Solubility $\left(\mathrm{mg} \mathrm{L}^{-1}\right)$ & Species & Exposure method & $\begin{array}{l}\text { Duration } \\
\text { (h) }\end{array}$ & $\begin{array}{c}\text { Measured } \\
\text { LC } \\
\left.\left(\mathrm{mg} \mathrm{L}_{5}\right)^{-1}\right) \\
\end{array}$ & $\begin{array}{c}\begin{array}{c}\text { Nominal } \\
\text { LC } \\
\left(\mathrm{mg} \mathrm{L}^{-1}\right)\end{array} \\
\end{array}$ & Classification \\
\hline Cyclohexene & $110-83-8$ & 82.14 & 2.99 & $2.50 \mathrm{E}+02$ & $\begin{array}{l}\text { Oryzias latipes } \\
\text { Poecilia reticulata }\end{array}$ & $\begin{array}{l}\text { semistatic } \\
\text { semistatic }\end{array}$ & $\begin{array}{l}96 \\
96\end{array}$ & $\begin{array}{l}5.80 \\
12.40\end{array}$ & & 1 \\
\hline 1,6-Octadien-3-ol, 3,7-dimethyl- & $78-70-6$ & 154.24 & 2.97 & $8.54 \mathrm{E}+02-1.59 \mathrm{E}+03$ & $\begin{array}{l}\text { Oncorhynchus mykiss } \\
\text { Leuciscus idus }\end{array}$ & $\begin{array}{l}\text { static } \\
\text { static }\end{array}$ & $\begin{array}{l}96 \\
96\end{array}$ & 27.80 & $22-46$ & 1 \\
\hline 2-methylpropyl 2-methyl-2-propenoate & $97-86-9$ & 142.20 & 2.95 & $4.70 \mathrm{E}+02$ & Oncorhynchus mykiss & flow-through & 96 & 20.00 & & 1 \\
\hline Styrene & $100-42-5$ & 104.15 & 2.95 & $3.00 \mathrm{E}+02$ & $\begin{array}{l}\text { Pimephales promelas } \\
\text { Pimephales promelas }\end{array}$ & $\begin{array}{l}\text { flow-through } \\
\text { flow-through }\end{array}$ & $\begin{array}{l}96 \\
96\end{array}$ & $\begin{array}{l}10.00 \\
4.02\end{array}$ & & 1 \\
\hline Benzene, 1,4-dichloro-2-nitro- & $89-61-2$ & & 2.93 & $9.50 \mathrm{E}+01$ & $\begin{array}{l}\text { Oryzias latipes } \\
\text { Leciscus idus }\end{array}$ & $\begin{array}{l}\text { semi-static } \\
\text { static }\end{array}$ & $\begin{array}{l}96 \\
48\end{array}$ & 5.40 & 4140.00 & 1 \\
\hline Benzene, (trichloromethyl)- & 98-07-7 & 195.48 & 2.92 & $1.00 \mathrm{E}+02$ & $\begin{array}{l}\text { Leuciscus idus } \\
\text { melanotus }\end{array}$ & static & 72 & & $>1000$ & 2 \\
\hline Dodecane-12-lactam & $947-04-6$ & 197.32 & 2.92 & $3.00 \mathrm{E}+02$ & $\begin{array}{l}\text { Cyprinus carpio } \\
\text { Leuciscus idus } \\
\text { Oryzias latipes }\end{array}$ & $\begin{array}{l}\text { semi-static } \\
\text { static }\end{array}$ & $\begin{array}{l}96 \\
48 \\
48\end{array}$ & $\begin{array}{l}63.00 \\
50.00 \\
68.40\end{array}$ & & 1 \\
\hline Guanidine, N,N'-bis(2-methylphenyl)- & $97-39-2$ & & 2.90 & $7.00 \mathrm{E}+01$ & Oryzias latipes & semi-static & 96 & 19.00 & & 1 \\
\hline Benzene, 2,4-dichloro-1-nitro- & 611-06-3 & & 2.90 & $2.00 \mathrm{E}+02$ & $\begin{array}{l}\text { Oryzias latipes } \\
\text { Pzable }\end{array}$ & semi-static & 96 & 13.00 & & 1 \\
\hline 1-Octanamine & $111-86-4$ & 129.25 & 2.90 & $1.00 \mathrm{E}+06$ & Pimephales promelas & & 96 & 5.19 & & 1 \\
\hline $\begin{array}{l}\text { Phenol, 4-(1-methylethenyl)- } \\
\text { 2-Propenoic acid, 2-ethyl-2-[(1-oxo-2-propenyl)oxy]methyl]-1,3- } \\
\text { propanediyl }\end{array}$ & $\begin{array}{l}4286-23-1 \\
15625-89-5\end{array}$ & 296.32 & $\begin{array}{l}2.90 \\
2.86\end{array}$ & $1.39 \mathrm{E}+03$ & Oryzias latipes & semi-static & 96 & 9.20 & & $\begin{array}{l}1 \\
4\end{array}$ \\
\hline 2-Butene, 1,3-dichloro- & $926-57-8$ & 125.00 & 2.84 & 3.63E+02 & $\begin{array}{l}\text { Brachydanio rerio } \\
\text { Pimephales promelas }\end{array}$ & $\begin{array}{l}\text { static } \\
\text { static }\end{array}$ & $\begin{array}{l}96 \\
96\end{array}$ & $\begin{array}{l}>11 \\
4.00\end{array}$ & & 1 \\
\hline Methane, tetrachloro- & $56-23-5$ & 153.82 & 2.83 & $8.46 \mathrm{E}+02$ & $\begin{array}{l}\text { Danio rerio } \\
\text { Oryzias latipes }\end{array}$ & $\begin{array}{l}\text { flow though } \\
\text { semi-static }\end{array}$ & $\begin{array}{l}96 \\
96\end{array}$ & $\begin{array}{l}24.00 \\
7.60\end{array}$ & & 1 \\
\hline Dibutylamine & $111-92-2$ & 129.25 & 2.83 & & $\begin{array}{l}\text { Oncorhynchus mykiss } \\
\text { Oncorhynchus mykiss }\end{array}$ & & $\begin{array}{l}96 \\
96\end{array}$ & & $\begin{array}{c}37.00 \\
5.50\end{array}$ & 1 \\
\hline $\begin{array}{l}\text { Phosphoric acid, 2,2-bis(chloromethyl)-1,3-propanediyl tetrakis(2- } \\
\text { chloroethyl) }\end{array}$ & $38051-10-4$ & & 2.83 & $2.32 \mathrm{E}+02$ & Oncorhynchus mykiss & semi-static & 96 & 52.00 & & 1 \\
\hline 1-Hexanamine, 2-ethyl- & $104-75-6$ & 129.24 & 2.82 & & $\begin{array}{l}\text { Leuciscus idus } \\
\text { Leuciscus idus }\end{array}$ & & 96 & & $\begin{aligned}> & 46.4-< \\
& 68.1 \\
> & 100-< \\
& 500\end{aligned}$ & 1 \\
\hline Butane, 1-chloro- & $109-69-3$ & & 2.82 & $3.70 \mathrm{E}+02$ & $\begin{array}{l}\text { Oryzias latipes } \\
\text { Orizias latipes }\end{array}$ & semi-static & $\begin{array}{l}96 \\
24\end{array}$ & 11.00 & 120.00 & 1 \\
\hline Dicyclopentadiene & $77-73-6$ & 132.20 & 2.78 & & $\begin{array}{l}\text { Orizias latipes } \\
\text { Orizias latipes } \\
\text { Orizias latipes }\end{array}$ & & $\begin{array}{l}48 \\
72 \\
96\end{array}$ & $\begin{array}{l}6.70 \\
6.70 \\
4.30\end{array}$ & & 1 \\
\hline 1-Hexanol, 2-ethyl- & $104-76-7$ & & 2.73 & & & & & & & 4 \\
\hline Butane, 2-methyl- & 78-78-4 & & 2.72 & & Oncorhynchus mykiss & & 96 & 3.10 & & 1 \\
\hline Hexanal, 2-ethyl- & $123-05-7$ & 128.22 & 2.71 & $4.00 \mathrm{E}+02$ & Oncorhynchus mykiss & & 96 & 1.94 & & 4 \\
\hline Aniline, 3,4-dichloro- & $95-76-1$ & 162.02 & 2.70 & $5.80 \mathrm{E}+02$ & $\begin{array}{l}\text { Pimephales promelas } \\
\text { Brachydanio rerio } \\
\text { Poecilia reticulata }\end{array}$ & & $\begin{array}{l}96 \\
96 \\
96\end{array}$ & $\begin{array}{l}6.99 \\
8.50 \\
8.70\end{array}$ & & 1 \\
\hline $\begin{array}{l}\text { 3H-Indol-3-one, 2-(1,3-dihydro-3-oxo-2H-indol-2-ylidene)-1,2- } \\
\text { dihydro- }\end{array}$ & $482-89-3$ & & 2.70 & $9.90 \mathrm{E}-01$ & Oryzias latipes & Semi-static & 96 & & $>1,000$ & 1 \\
\hline 2-methylpropyl 2-methyl-2-propanoate & $97-85-8$ & 144.21 & 2.68 & $5.20 \mathrm{E}+02$ & $\begin{array}{l}\text { Oncorhynchus mykiss } \\
\text { Oncorhynchus mykiss }\end{array}$ & $\begin{array}{l}\text { static renewal } \\
\text { flow-through }\end{array}$ & $\begin{array}{l}96 \\
96\end{array}$ & $\begin{array}{l}8.27 \\
>4.2\end{array}$ & & 1 \\
\hline $\begin{array}{l}\text { 2-Butene, 2-methyl- } \\
\text { Benzyl chloride }\end{array}$ & $\begin{array}{l}513-35-9 \\
100-44-7\end{array}$ & 70.14 & $\begin{array}{l}2.67 \\
2.66\end{array}$ & $\begin{array}{l}1.93 \mathrm{E}+02 \\
1.20 \mathrm{E}+03\end{array}$ & $\begin{array}{l}\text { Oncorhynchus mykiss } \\
\text { Oryzias latipes } \\
\text { Pimephales promelas }\end{array}$ & $\begin{array}{l}\text { Semi-static } \\
\text { semi-static } \\
\text { flow-through }\end{array}$ & $\begin{array}{l}96 \\
96 \\
96\end{array}$ & 4.99 & 4.20 & $\begin{array}{l}1 \\
1\end{array}$ \\
\hline Toluene & $108-88-3$ & 92.14 & 2.65 & $5.15 \mathrm{E}+02$ & $\begin{array}{l}\text { Carassius auratus } \\
\text { Lepomis macrochiru }\end{array}$ & $\begin{array}{l}\text { flow-through } \\
\text { static }\end{array}$ & $\begin{array}{l}96 \\
96\end{array}$ & 22.80 & 13.00 & 1 \\
\hline $\begin{array}{l}\text { Hexanoic acid, 2-ethyl- } \\
\text { Diisobutylamine }\end{array}$ & $\begin{array}{l}149-57-5 \\
110-96-3\end{array}$ & $\begin{array}{l}144.22 \\
129.25\end{array}$ & $\begin{array}{l}2.64 \\
2.63\end{array}$ & & Leuciscus idus & & 96 & & 26.00 & $\begin{array}{l}4 \\
1\end{array}$ \\
\hline $\begin{array}{l}\text { Dehydrolinalool } \\
\text { Dehol }\end{array}$ & $29171-20-8$ & 152.24 & 2.61 & $2.45 \mathrm{E}+03$ & Lenctschs taus & & 然 & & & 4 \\
\hline $\begin{array}{l}\text { Silane, dichloroethenylmethyl- } \\
\text { Diisobutyl ketone }\end{array}$ & $\begin{array}{l}124-70-9 \\
108-83-8\end{array}$ & & $\begin{array}{l}2.60 \\
2.56\end{array}$ & $\begin{array}{l}8.02 \mathrm{E}+02 \\
4.30 \mathrm{E}+02\end{array}$ & Oncorhynchus mykiss & semi-static & & & 140.00 & $\begin{array}{l}4 \\
1\end{array}$ \\
\hline 2,2'-dimethyl-4,4'-methylenebis(cyclohexylamine) & $\begin{array}{l}108-80-8 \\
6864-37-5\end{array}$ & & 2.51 & $\begin{array}{l}4.00 \mathrm{E}+02 \\
3.60 \mathrm{E}+03\end{array}$ & $\begin{array}{l}\text { Leuciscus idus } \\
\text { Leiss }\end{array}$ & static & 96 & & $>22-46.4$ & 3 \\
\hline Silane, dichloro(chloromethyl)methyl- & $1558-33-4$ & & 2.50 & $4.85 \mathrm{E}+02$ & & & & & & 4 \\
\hline
\end{tabular}


(Table S7. continued)

\begin{tabular}{|c|c|c|c|c|c|c|c|c|c|c|}
\hline Chemicals name & CAS Reg. no & $\begin{array}{c}\text { Molecular } \\
\text { weight } \\
\left(\mathrm{g} \mathrm{mol}^{-1}\right)\end{array}$ & $\log \mathrm{K}_{\mathrm{ow}}$ & Solubility $\left(\mathrm{mg} \mathrm{L}^{-1}\right)$ & Species & Exposure method & $\begin{array}{l}\text { Duration } \\
\text { (h) }\end{array}$ & $\begin{array}{c}\text { Measured } \\
\mathrm{LC}_{50} \\
\left(\mathrm{mg} \mathrm{L}^{-1}\right) \\
\end{array}$ & $\begin{array}{c}\text { Nominal } \\
\mathrm{LC}_{50} \\
\left(\mathrm{mg} \mathrm{L}^{-1}\right) \\
\end{array}$ & Classification \\
\hline Silane, trichloroethyl- & $115-21-9$ & 163.51 & 2.50 & $9.87 \mathrm{E}+02$ & $\begin{array}{l}\text { Oncorhynchus mykiss } \\
\text { Oncorynnchus mykiss }\end{array}$ & $\begin{array}{l}\text { semi-static } \\
\text { static }\end{array}$ & $\begin{array}{l}96 \\
96\end{array}$ & & $\begin{array}{l}271.00 \\
>126\end{array}$ & 1 \\
\hline Silane, chlorotrimethyl- & $75-77-4$ & & 2.48 & $1.01 \mathrm{E}+03$ & Oncorhynchus mykiss & static & 96 & $>126$ & & 3 \\
\hline Ethane, 1,1,1-trichloro- & $71-55-6$ & & 2.47 & $3.00 \mathrm{E}+02$ & Pimephales promelas & & 96 & 52.80 & & 1 \\
\hline \multirow{2}{*}{$\begin{array}{l}\text { Benzoic acid, 4-methyl- } \\
\text { bicis }\end{array}$} & $99-94-5$ & & 2.44 & $3.49 \mathrm{E}+02$ & Oryzias latipes & semi-static & 96 & 64.00 & & 1 \\
\hline & & & & & Brachydanio rerio & flow-through & 48 & 60.00 & & \\
\hline Ethene, trichloro- & $79-01-6$ & 131.39 & 2.42 & $1.10 \mathrm{E}+03$ & $\begin{array}{l}\text { Pimephales promelas } \\
\text { Orvzias latives }\end{array}$ & $\begin{array}{l}\text { flow-through } \\
\text { static }\end{array}$ & $\begin{array}{l}96 \\
48\end{array}$ & 40.70 & 7900 & 1 \\
\hline Isoprene & $\begin{array}{l}78-79-5 \\
106-98-9\end{array}$ & 68.12 & 2.42 & $6.42 \mathrm{E}+02$ & Oncorhynchus mykiss & semi-static & 96 & 7.43 & & 1 \\
\hline 1-Butene, 2,3,4-trichloro- & $\begin{array}{l}100-98-9 \\
2431-50-7\end{array}$ & 159.44 & 2.40 & $6.00 \mathrm{E}+02$ & & & & & & $\begin{array}{l}4 \\
4\end{array}$ \\
\hline 2-Ethylhexyl mercaptoacetate & $7659-86-1$ & & 2.40 & $4.73 \mathrm{E}+00$ & Leuciscus idus & static & 48 & & 9.00 & 2 \\
\hline Hexanoyl chloride, 2-ethyl- & $760-67-8$ & 162.66 & 2.40 & $5.93 \mathrm{E}+02$ & Brachydanio rerio & static & 96 & & 66.30 & 1 \\
\hline Hept-5-en-2-one, 6-methyl- & $110-93-0$ & 126.20 & 2.40 & $3.02 \mathrm{E}+03$ & $\begin{array}{l}\text { Pimephales promelas } \\
\text { Leuciscus idus }\end{array}$ & $\begin{array}{l}\text { flow through } \\
\text { static }\end{array}$ & $\begin{array}{l}96 \\
96\end{array}$ & 85.70 & 68.10 & 1 \\
\hline \multirow{2}{*}{$\begin{array}{l}\text { Silane, trichloroethenyl- } \\
\text { Benzene, 1-chloro-4-nitro- }\end{array}$} & $75-94-5$ & & 2.40 & $1.34 \mathrm{E}+03$ & & & & & 08.10 & 4 \\
\hline & $100-00-5$ & 157.55 & 2.39 & $2.43 \mathrm{E}+02$ & $\begin{array}{l}\text { Brachydanio rerio } \\
\text { Pimephales promelas }\end{array}$ & $\begin{array}{l}\text { flow-through } \\
\text { flow-through }\end{array}$ & 96 & $\begin{array}{l}14.36 \\
20.40\end{array}$ & & 1 \\
\hline Ethane, 1,1,2,2-tetrachloro- & $79-34-5$ & 167.85 & 2.39 & $2.87 \mathrm{E}+03$ & $\begin{array}{l}\text { Pryzizas latipes } \\
\text { Jordanelas } \\
\text { Jordanella floridae } \\
\text { Joridae }\end{array}$ & $\begin{array}{l}\text { sem-nistaugn } \\
\text { semi-stic } \\
\text { flow-through } \\
\text { semi-static }\end{array}$ & $\begin{array}{l}48 \\
96 \\
96\end{array}$ & 185.00 & $\begin{array}{l}31.00 \\
26.80\end{array}$ & 1 \\
\hline Butyl acrylate & $141-32-2$ & 128.17 & 2.38 & $2.00 \mathrm{E}+03$ & $\begin{array}{l}\text { Cyprinodon variegatus } \\
\text { Salmo gairdneri }\end{array}$ & $\begin{array}{l}\text { flow through } \\
\text { flow through }\end{array}$ & $\begin{array}{l}96 \\
96\end{array}$ & $\begin{array}{l}2.10 \\
5.20\end{array}$ & & 1 \\
\hline Benzoic acid, 3-methyl- & 99-04-7 & 136.15 & 2.37 & $1.00 \mathrm{E}+03$ & $\begin{array}{l}\text { Oryzias latipes } \\
\text { Pimephales promelas }\end{array}$ & $\begin{array}{l}\text { semi-static } \\
\text { flow-through }\end{array}$ & $\begin{array}{l}96 \\
96\end{array}$ & 49.70 & 82.00 & 1 \\
\hline Toluene, 4-nitro- & $99-99-0$ & 137.14 & 2.37 & $3.45 \mathrm{E}+02$ & $\begin{array}{l}\text { Pimphales promelas } \\
\text { Carassius auratus } \\
\text { Oryzias latipes } \\
\text { Cyprinus carpio }\end{array}$ & $\begin{array}{l}\text { flow-through } \\
\text { static } \\
\text { semi-static } \\
\text { semi-static }\end{array}$ & $\begin{array}{l}96 \\
48 \\
48 \\
96\end{array}$ & $\begin{array}{l}49.70 \\
10.50 \\
74.00 \\
40.00\end{array}$ & & 1 \\
\hline 1-Butene, 3,4-dichloro- & $760-23-6$ & 125.00 & 2.37 & $1.10 \mathrm{E}+03$ & $\begin{array}{l}\text { Oryzias latipes } \\
\text { Pimephales romelas }\end{array}$ & $\begin{array}{l}\text { flow-through } \\
\text { flow-thropugh }\end{array}$ & 96 & $\begin{array}{l}27.00 \\
7.17\end{array}$ & & 1 \\
\hline \multirow{7}{*}{$\begin{array}{l}\text { Phenol, 4,4'-sulfonylbis- } \\
\text { Phenol, 2,6-dimethyl- } \\
\text { Propene, 2-methyl- } \\
\text { 2-Butene, (2E)- } \\
\text { 2-Butene, (2Z)- } \\
\text { tert-Butyl acrylate }\end{array}$} & $80-09-1$ & & 2.36 & $7.70 \mathrm{E}+02$ & $\begin{array}{l}\text { Prmephales promelas } \\
\text { Oryzias latipes }\end{array}$ & semi-static & 96 & & $>100$ & 3 \\
\hline & $576-26-1$ & 122.17 & 2.36 & & & & & & & 4 \\
\hline & $115-11-7$ & 56.11 & 2.34 & $2.63 \mathrm{E}-01$ & & & & & & 4 \\
\hline & $624-64-6$ & 56.11 & 2.33 & $2.65 \mathrm{E}+02$ & fish & & 96 & & 21.00 & 1 \\
\hline & $590-18-1$ & 56.11 & 2.33 & $7.00 \mathrm{E}+02$ & & & & & & 4 \\
\hline & $1663-39-4$ & & 2.32 & $2.00 \mathrm{E}+03$ & Leuciscus idus & & 96 & & 1.68 & 1 \\
\hline & & & & & $\begin{array}{l}\text { Poecilia reticulata } \\
\text { Poecilia reticulata }\end{array}$ & & $\begin{array}{l}96 \\
24\end{array}$ & $\begin{array}{l}30.10 \\
29.00\end{array}$ & & \\
\hline \multirow[t]{3}{*}{ Toluene, 2-nitro- } & $88-72-2$ & 137.14 & 2.30 & $4.37 \mathrm{E}+02$ & Brachydario rerio & & $\begin{array}{l}96 \\
48\end{array}$ & $\begin{array}{r}64.90 \\
2900\end{array}$ & & 1 \\
\hline & & & & & $\begin{array}{l}\text { Pimephales promelas } \\
\text { Pimas }\end{array}$ & & 96 & 37.10 & & \\
\hline & & & & & Oryzias latipes & & 48 & 86.00 & & \\
\hline \multirow{6}{*}{$\begin{array}{l}\text { Aniline, 4-(1-methylethyl)- } \\
\text { Ethane, 1,1-dichloro-1-fluoro- }\end{array}$} & 99-88-7 & & 2.30 & $2.39 \mathrm{E}+03$ & Oryzias latipes & semi-static & 96 & 46.00 & & 1 \\
\hline & $1717-00-6$ & & 2.30 & $4.00 \mathrm{E}+03$ & Brachydanio rerio & & 96 & & 126.00 & 1 \\
\hline & & & & & Lepomis macrochirus & & 24 & 260.00 & & \\
\hline & & & & & Lepomis macrochirus & & 48 & 160.00 & & \\
\hline & & & & & Lepomis macrochirus & & 96 & 100.00 & & \\
\hline & & & & & Pimephales promelas & & 24 & 320.00 & & \\
\hline \multirow[t]{4}{*}{ 2-Propenamide } & 79-06-1 & 131.50 & 2.29 & $1.10 \mathrm{E}+03$ & Pimephales promelas & & 48 & 230.00 & & 1 \\
\hline & & & & & Pimephales promelas & & 96 & 120.00 & & \\
\hline & & & & & Oncorhynchus mykiss & & 24 & 370.00 & & \\
\hline & & & & & Oncorhynchus mykiss & & 48 & 240.00 & & \\
\hline 1-Butanethiol & $109-79-5$ & & 2.28 & $595 \mathrm{E}+02$ & Oncorhynchus mykiss & & 96 & 110.00 & & 4 \\
\hline 2-Picoline, 5-ethyl- & $104-90-5$ & 121.18 & $2.27-2.52$ & $1.20 \mathrm{E}+04$ & Salmo gairdneri & static & 96 & $55.6-100$ & & 1 \\
\hline Butane, 1-isocyanato- & $111-36-4$ & 99.13 & 2.26 & 202900 & $\begin{array}{l}\text { Pimephales promelas } \\
\text { Menidia beryllina }\end{array}$ & $\begin{array}{l}\text { flow-through } \\
\text { static }\end{array}$ & $\begin{array}{l}96 \\
96\end{array}$ & 81.10 & 24.00 & 1 \\
\hline
\end{tabular}


(Table S7. continued)

\begin{tabular}{|c|c|c|c|c|c|c|c|c|c|c|}
\hline Chemicals name & CAS Reg. no & $\begin{array}{c}\text { Molecular } \\
\text { weight } \\
\left(\mathrm{g} \mathrm{mol}^{-1}\right)\end{array}$ & $\log \mathrm{K}_{\mathrm{ow}}$ & Solubility $\left(\mathrm{mg} \mathrm{L}^{-1}\right)$ & Species & Exposure method & $\begin{array}{l}\text { Duration } \\
\text { (h) }\end{array}$ & $\begin{array}{c}\text { Measured } \\
\mathrm{LC}_{50} \\
\left(\mathrm{mg} \mathrm{L}^{-1}\right) \\
\end{array}$ & $\begin{array}{c}\text { Nominal } \\
\text { LC } \\
\left(\mathrm{mg} \mathrm{L}_{5-1}\right) \\
\end{array}$ & Classification \\
\hline \multirow{3}{*}{ Dimethyl terephthalate } & $120-61-6$ & 194.18 & 2.25 & $1.90 \mathrm{E}+01$ & $\begin{array}{l}\text { Pimephales promelas } \\
\text { Pimephales promelas }\end{array}$ & $\begin{array}{l}\text { static } \\
\text { static }\end{array}$ & $\begin{array}{l}96 \\
96\end{array}$ & 9.60 & 45.00 & 1 \\
\hline & $120-01-0$ & 194.18 & 2.25 & $1.90 \mathrm{DE}+01$ & $\begin{array}{l}\text { Pimephales promelas } \\
\text { Pimephes }\end{array}$ & & 96 & & 14.20 & 1 \\
\hline & & & & & Brachydanio rerio & flow-through & 96 & 34.80 & & \\
\hline \multirow{3}{*}{ Benzene, 1-chloro-2-nitro- } & $88-73-3$ & 15755 & 224 & $441 \mathrm{~F}+0 ?$ & Oryzias latipes & semi-static & 48 & 28.00 & & 1 \\
\hline & $88-13-3$ & 157.53 & 2.24 & 4.41E+02 & Cyprinus carpio & semi-static & 96 & 25.50 & & 1 \\
\hline & & & & & Poecilia reticulata & semi-static & 96 & 30.00 & & \\
\hline Silane, dichlorodimethyl- & $75-78-5$ & 129.06 & 2.24 & $1.75 \mathrm{E}+03$ & Oncorhynchus mykiss & semi-static & 96 & 271.00 & \multirow{3}{*}{$>=519$} & 1 \\
\hline & $106-63-8$ & & 2.22 & $2.00 \mathrm{E}+03$ & $\begin{array}{l}\text { Brachydanio rerio } \\
\text { Pimephales promelas }\end{array}$ & $\begin{array}{l}\text { static } \\
\text { flow-through }\end{array}$ & $\begin{array}{l}96 \\
96\end{array}$ & 2.09 & & 1 \\
\hline 1,2-ethanediyl 2-methyl-2-propenoate & $97-90-5$ & & 2.21 & & & & & & & 4 \\
\hline o-Cresol & $95-48-7$ & 108.14 & 2.20 & $2.60 \mathrm{E}+04$ & $\begin{array}{l}\text { Oncorhynchus mykiss } \\
\text { Salmo trutta }\end{array}$ & flow-through & $\begin{array}{l}96 \\
96\end{array}$ & $\begin{array}{l}8.40 \\
6.20\end{array}$ & & 1 \\
\hline Chloroprene & $126-99-8$ & & 2.20 & $2.56 \mathrm{E}+02-4.80 \mathrm{E}+02$ & Lepomis macrochirus & flow -through & 96 & 245.00 & & 1 \\
\hline 3,4-Dimethylaniline & $95-64-7$ & & 2.17 & $2.46 \mathrm{E}+03$ & Oryzias latipes & semi-static & 96 & $>97.9$ & & 3 \\
\hline Aniline, 2,6-dimethyl- & $87-62-7$ & & 2.17 & $6.80 \mathrm{E}+03-7.14 \mathrm{E}+03$ & Oryzias latipes & semi-static & 96 & $>97.9$ & & 3 \\
\hline 2,3-Dimethylaniline & $87-59-2$ & & 2.17 & $6.60 \mathrm{E}+03$ & Oryzias latipes & semi-static & 96 & $>94$ & & 3 \\
\hline 3,5-Dimethylaniline & $108-69-0$ & 121.18 & 2.17 & $4.60 \mathrm{E}+03$ & Oryzias latipes & semi-static & 96 & & 33.90 & 1 \\
\hline 2-Propenoic acid, 2-methyl-, 2-propenyl ester & 0096-05-9 & & 2.15 & $2.20 \mathrm{E}+03$ & Pimephales promelas & flow -through & 96 & 0.61 & & 1 \\
\hline Stannane, trichlorooctyl- & $3091-25-6$ & 338.29 & 2.14 & $3.30 \mathrm{E}-01$ & Brachydanio rerio & static & 96 & $>0.33$ & & 3 \\
\hline 2-Propanethiol, 2-methyl- & $75-66-1$ & & 2.14 & $2.05 \mathrm{E}+03$ & Oncorhynchus mykiss & semi-static & 96 & 34.00 & & 1 \\
\hline \multirow{2}{*}{ Stannane, dibutyldichloro- } & $683-18-1$ & 303.84 & 2.13 & $3.20 \mathrm{E}-01$ & Brachydanio rerio & semistatic & 96 & $>4$ & & 3 \\
\hline & & & & & Pimephales promelas & flow-through & 96 & 15.60 & & \\
\hline \multirow[t]{2}{*}{ Benzene } & $71-43-2$ & 78.11 & 2.13 & $1.80 \mathrm{E}+03$ & Pimephales promelas & flow-through & 96 & 15.10 & & 1 \\
\hline & & & & & Poecilia reticulata & semi-static & 96 & 28.60 & & \\
\hline 2-Butenedioic acid (2E)-, diethyl ester & $623-91-6$ & & 2.12 & $3.10 \mathrm{E}+03$ & Oryzias latipes & semistatic & 96 & & 2.40 & 1 \\
\hline 1-Propene, hexafluoro- & $116-15-4$ & 150.02 & 2.12 & & & & & & & 4 \\
\hline \multirow{2}{*}{$\begin{array}{l}\text { Phenol, 3-methyl-4-nitro- } \\
\text { Pallo }\end{array}$} & 2581-34-2 & & 2.12 & $1.30 \mathrm{E}+01$ & Oryzias latipes & semi-static & 96 & & 9.80 & 1 \\
\hline & & & & & Oryzias latipes & semi-static & 48 & 6.90 & & \\
\hline \multirow[t]{2}{*}{ 1-Propene, 3-chloro- } & $107-05-1$ & & 2.10 & $3.60 \mathrm{E}+03$ & Carassius auratus & static & 96 & & 21.00 & 1 \\
\hline & & & & & Lebistes reticulatus & static & 96 & & 51.00 & \\
\hline \multirow[t]{2}{*}{ 3-(Trimethoxysilyl)propyl methacrylate } & $2530-85-0$ & & 2.10 & $8.26 \mathrm{E}+01$ & Brachydanio rerio & semi-static & 96 & & $>100$ & 3 \\
\hline & & & & & Brachydanio rerio & & 96 & 0.01 & & \\
\hline \multirow{4}{*}{ Stannane, tributylchloro- } & $1461-22-9$ & & 2.07 & $6.00 \mathrm{E}+00-1.00 \mathrm{E}+01$ & Menidia beryllina & & 96 & 0.00 & & 1 \\
\hline & & & & & Menidia menidia & & 96 & 0.01 & & \\
\hline & & & & & Brevoortia tyrannus & & 96 & 0.00 & & \\
\hline & & & & & Cyprinodon variegatus & semi-static & 96 & 8.60 & & \\
\hline \multirow[t]{2}{*}{ Acrylonitrile } & $107-13-1$ & 53.06 & 2.05 & $7.35 \mathrm{E}+04$ & Lepomis machrochirus & static & 96 & & 10.00 & 1 \\
\hline & & & & & Brachydanio rerio & flow-through & 48 & 15.00 & & \\
\hline Silane, triethoxy(2-methylpropyl)- & $17980-47-1$ & & 2.03 & $8.60 \mathrm{E}+01$ & Salmo gairdneri & semi-static & 96 & & 85.00 & 1 \\
\hline 1-Hexanol & $111-27-3$ & 102.18 & 2.03 & $5.90 \mathrm{E}+03$ & Pimephales promelas & flow-through & 96 & 97.00 & & 1 \\
\hline Silanamine, trimethyl-N-trimethylsilyl- & $999-97-3$ & 161.40 & 2.02 & $7.61 \mathrm{E}+02$ & Brachydanio rerio & semi-static & 96 & 88.00 & & 1 \\
\hline \multirow{2}{*}{ Cyclohexylamine, N,N-dimethyl- } & 98-94-2 & & 2.01 & $1.34 \mathrm{E}+04$ & Leuciscus idus & static & 96 & & 31.60 & 1 \\
\hline & & & & & $\begin{array}{l}\text { Oncorhynchus mykiss } \\
\text { Oncorbnychus }\end{array}$ & static & 96 & 28.00 & & 1 \\
\hline \multirow[t]{2}{*}{ Silane, trichloromethyl- } & $75-79-6$ & & 2.01 & & $\begin{array}{l}\text { Oncorhynchus mykiss } \\
\text { Brachydanio rerio }\end{array}$ & & $\begin{array}{l}96 \\
96\end{array}$ & $\begin{array}{l}271.00 \\
>=519\end{array}$ & & 1 \\
\hline & & & & & Oncorhynchus mykiss & & 96 & $>126$ & & \\
\hline \multirow{2}{*}{ 2-Naphthol } & $135-19-3$ & & $201-284$ & $600 \mathrm{E}+02-900 \mathrm{E}+02$ & Gadus morrhua & static & 96 & & $>3$ & 3 \\
\hline & & & & $6.00 \mathrm{E}+02-9.00 \mathrm{E}+02$ & Pimephales promelas & static & 96 & & 3.46 & 3 \\
\hline Propane, 1,2-dichloro- & $78-87-5$ & 112.99 & 2.00 & $2.80 \mathrm{E}+03$ & Pimephales promelas & flow through & 96 & 139.00 & & 1 \\
\hline \multirow{2}{*}{$\begin{array}{l}\text { 1,3-Butadiene } \\
\text { Propane, 1,2,3-trichloro- }\end{array}$} & $106-99-0$ & 54.09 & 1.99 & $7.35 \mathrm{E}+02$ & Pimephales promelas & flow through & 96 & 140.00 & & 4 \\
\hline & $96-18-4$ & & $1.98-2.54$ & $1.75 \mathrm{E}+04$ & Pimephales promelas & flow-through & 96 & 66.50 & & 1 \\
\hline \multirow[t]{2}{*}{ 2,4-Dinitrotoluene } & $121-14-2$ & 182.14 & 1.98 & & Ictalurus punctatus & flow through & 96 & 32.00 & & 1 \\
\hline & $106-93-4$ & & 1.96 & $3.91 \mathrm{E}+03$ & $\begin{array}{l}\text { Lepomis macrochirus } \\
\text { Oryzias latipes }\end{array}$ & $\begin{array}{l}\text { flow through } \\
\text { flow-through }\end{array}$ & $\begin{array}{l}96 \\
96\end{array}$ & $\begin{array}{l}16.00 \\
32.10\end{array}$ & & 1 \\
\hline Ethane, 1,2-dibromo- & $106-93-4$ & & 1.96 & $3.91 \mathrm{E}+03$ & Pimephales promelas & & 96 & 4.30 & & 1 \\
\hline
\end{tabular}


(Table S7. continued)

\begin{tabular}{|c|c|c|c|c|c|c|c|c|c|c|}
\hline Chemicals name & CAS Reg. no & $\begin{array}{c}\text { Molecular } \\
\text { weight } \\
\left(\mathrm{g} \mathrm{mol}^{-1}\right)\end{array}$ & $\log \mathrm{K}_{\mathrm{ow}}$ & Solubility $\left(\mathrm{mg} \mathrm{L}^{-1}\right)$ & Species & Exposure method & $\begin{array}{l}\text { Duration } \\
\text { (h) }\end{array}$ & $\begin{array}{c}\text { Measured } \\
\mathrm{LC}_{50} \\
\left(\mathrm{mg} \mathrm{L}^{-1}\right) \\
\end{array}$ & $\begin{array}{c}\text { Nominal } \\
\mathrm{LC}_{50} \\
\left(\mathrm{mg} \mathrm{L}^{-1}\right) \\
\end{array}$ & Classification \\
\hline \multirow{3}{*}{ Phenol, 3-methyl- } & \multirow{3}{*}{$108-39-4$} & \multirow{3}{*}{108.14} & \multirow{3}{*}{1.96} & \multirow{3}{*}{$2.27 \mathrm{E}+04$} & Pimephales promelas & flow-through & 96 & 55.90 & \multirow{4}{*}{$\begin{array}{l}8.40 \\
7.60\end{array}$} & \\
\hline & & & & & Oncorhynchus m & flow through & 96 & 8.90 & & 1 \\
\hline & & & & & $\begin{array}{l}\text { Salmo trutta } \\
\text { Salvelinus fontinalis }\end{array}$ & $\begin{array}{l}\text { static } \\
\text { static }\end{array}$ & $\begin{array}{l}96 \\
96\end{array}$ & & & \\
\hline 1,2,4-Benzenetricarboxylic acid, cyclic-1,2-anhydride & $552-30-7$ & \multirow{3}{*}{136.50} & 1.95 & $2.10 \mathrm{E}+04$ & $\begin{array}{l}\text { Leuciscus idus } \\
\text { melanotus }\end{array}$ & static & 96 & $\begin{array}{c}\text { not } \\
\text { determined }\end{array}$ & & 3 \\
\hline \multirow{3}{*}{ Ethane, 2-chloro-1,1,1,2-tetrafluoro- } & \multirow{3}{*}{ 2837-89-0 } & & \multirow{3}{*}{1.94} & \multirow{3}{*}{$1.45 \mathrm{E}+03$} & Brachydanio rerio & & 96 & & \multirow{3}{*}{$\begin{array}{l}126.00 \\
220.00\end{array}$} & \\
\hline & & & & & Poecilia reticulata & & 96 & & & 1 \\
\hline & & \multirow{3}{*}{108.14} & & & Pimephales promelas & flow-through & 96 & 28.60 & & \multirow{3}{*}{1} \\
\hline \multirow[t]{2}{*}{ Phenol, 4-methyl- } & \multirow[t]{2}{*}{$106-44-5$} & & \multirow[t]{2}{*}{1.94} & \multirow{2}{*}{$2.15 \mathrm{E}+04$} & Oncorhynchus mykiss & flow-through & 96 & 7.90 & \multirow[b]{2}{*}{4.40} & \\
\hline & & & & & Salmo trutta & static & 96 & 390 & & \\
\hline Methane, bromo- & 74-83-9 & & 1.94 & $1.61 \mathrm{E}+04$ & Lepomis macrochirus & & 96 & 3.90 & 1100 & 1 \\
\hline & & & & & Oryzias latipes & semi-static & 96 & 0.70 & 111.00 & 1 \\
\hline Silane, chlorodimethyl- & $1066-35-9$ & & 1.93 & & & & & & & 4 \\
\hline Ethane, 1,1,2-trichloro- & $79-00-5$ & & 1.89 & $2.87 \mathrm{E}+03$ & $\begin{array}{l}\text { Pimephales promelas } \\
\text { Oryzias latipes } \\
\text { Jordanella floridae }\end{array}$ & $\begin{array}{l}\text { flow through } \\
\text { semistatic } \\
\text { flow through }\end{array}$ & $\begin{array}{l}96 \\
48 \\
96\end{array}$ & $\begin{array}{c}20-20.9 \\
18.50\end{array}$ & 31.00 & 1 \\
\hline $\begin{array}{l}\text { 2-Propenoic acid, 2-methyl-,1,2-ethanediylbis(oxy-2,1- } \\
\text { ethanediyl)ester }\end{array}$ & $109-16-0$ & 286.33 & 1.88 & & & & & & & 4 \\
\hline Benzoic acid & $65-85-0$ & & 1.88 & $2.90 \mathrm{E}+03$ & $\begin{array}{l}\text { Lepomis macrochirus } \\
\text { Salmo gairdneri }\end{array}$ & $\begin{array}{l}\text { static } \\
\text { static }\end{array}$ & $\begin{array}{l}96 \\
96\end{array}$ & & $\begin{array}{l}44.60 \\
47.30\end{array}$ & 1 \\
\hline Ethyl methacrylate & $97-63-2$ & & 1.87 & $4.69 \mathrm{E}+03$ & $\begin{array}{l}\text { Salmo gairdneri } \\
\text { Brachydanio revio }\end{array}$ & flow-through & 96 & $\begin{array}{l}100.00 \\
567.20\end{array}$ & & 1 \\
\hline $\begin{array}{l}\text { Aniline, 2-nitro- } \\
\text { 2-Butene }\end{array}$ & $\begin{array}{l}88-74-4 \\
107-01-7\end{array}$ & 56.10 & $\begin{array}{l}1.85 \\
1.85\end{array}$ & $1.17 \mathrm{E}+03$ & $\begin{array}{l}\text { Brachydanio rerio } \\
\text { Brachydanio rerio }\end{array}$ & $\begin{array}{l}\text { static } \\
\text { semi-static }\end{array}$ & $\begin{array}{l}96 \\
96\end{array}$ & $\begin{array}{c}567.20 \\
19.50\end{array}$ & & 1 \\
\hline & & & & & Lepomis macrochirus & & 96 & 191.00 & & \\
\hline Methacrylate, methyl- & $80-62-6$ & & 1.83 & $1.60 \mathrm{E}+04$ & Lepomis macrochirus & & 72 & 264.00 & & 1 \\
\hline & & & & & Oncorhynchus mykiss & & 96 & & & \\
\hline 2,5-Dimethylaniline & $95-78-3$ & & 1.83 & $6.60 \mathrm{E}+03$ & Oryzias latipes & semi-static & 96 & $>110$ & & 3 \\
\hline p-Phenylenediamine, $\mathrm{N}$-phenyl- & $101-54-2$ & & 1.82 & $5.04 \mathrm{E}+02$ & Danio rerio & static & 96 & & 1.90 & 1 \\
\hline Tripropylene glycol diacrylate & $42978-66-5$ & 300.35 & 1.82 & & & & & & & 4 \\
\hline Butyl acetate & $123-86-4$ & & 1.82 & $1.40 \mathrm{E}+04$ & Pimephales promelas & flow through & 96 & 18.00 & & 1 \\
\hline 1-Propanethiol & $107-03-9$ & & 1.81 & $1.90 \mathrm{E}+03$ & Pimephales promelas & semi-static & 96 & 1.30 & & 1 \\
\hline Thiophene & $110-02-1$ & & 1.81 & $3.02 \mathrm{E}+03$ & Oryzias latipes & semi-static & 96 & 31.00 & & 1 \\
\hline 2-Methylpropyl acetate & $110-19-0$ & & 1.78 & $6.00 \mathrm{E}+03$ & & & & & & 4 \\
\hline 1-Propene & $115-07-1$ & & 1.77 & $2.00 \mathrm{E}+02$ & & & & & & 4 \\
\hline Isophthalic acid & $121-91-5$ & & 1.76 & $1.30 \mathrm{E}+02$ & $\begin{array}{l}\text { Leuciscus idus } \\
\text { melanotus }\end{array}$ & static & 96 & & - & 3 \\
\hline Benzaldehyde, 4-methoxy- & $123-11-5$ & & 1.76 & $4.40 \mathrm{E}+03$ & Oryzias latipes & semi-static & 96 & 40.00 & 15000 & 1 \\
\hline Ethane, 1,1,1-trifluoro- & $420-46-2$ & & 1.74 & $7.61 \mathrm{E}+02$ & $\begin{array}{l}\text { Leuciscus idus } \\
\text { Oncorhycus mykiss }\end{array}$ & $\begin{array}{l}\text { Static } \\
\text { flow-through }\end{array}$ & $\begin{array}{l}96 \\
96\end{array}$ & $>40$ & 150.00 & 3 \\
\hline Disulfide, bis(dimethylthiocarbamoyl)- & $137-26-8$ & & 1.73 & $1.80 \mathrm{E}+01$ & Oryzias latipes & semi-static & 96 & 0.17 & & 1 \\
\hline $\begin{array}{l}\text { Cyanuric chloride } \\
\text { Conth }\end{array}$ & $108-77-0$ & 184.41 & 1.70 & $4.40 \mathrm{E}+02$ & & & & & & 4 \\
\hline Silane, dichloromethyl- & $75-54-7$ & & 1.70 & & & & & & & 4 \\
\hline & & & & & Pimephales promelas & flow-through & 96 & & 4.20 & \\
\hline Guanidine, 1,3-diphenyl- & $102-06-7$ & & 1.69 & $4.75 \mathrm{E}+02$ & Oncorhynchus mykiss & static & 96 & & 11.00 & 1 \\
\hline & & & & & Lepomis macrochirus & static & 96 & & 9.60 & \\
\hline Methane, dibromo- & $74-95-3$ & & 1.68 & $9.00 \mathrm{E}+03$ & Rainbow Trout & & 96 & & 45.00 & 1 \\
\hline Butanoic acid, 2-ethyl & $88-09-5$ & & 1.68 & $1.70 \mathrm{E}+04$ & Oryzias latipes & & 96 & $>50$ & & 3 \\
\hline 2,4-Xylidine & $95-68-1$ & & 1.68 & $6.07 \mathrm{E}+03$ & & & & & & 4 \\
\hline 2-Pentanol, 4-methyl- & $108-11-2$ & 102.17 & 1.68 & $1.64 \mathrm{E}+04$ & Oncorhynchus mykiss & semi-static & 96 & 359.00 & & 1 \\
\hline 2-Cyclohexen-1-one, 3,5,5-trimethyl- & $78-59-1$ & & 1.67 & $1.45 \mathrm{E}+04$ & Cyprinodon variegatus & & 96 & 140.00 & & 1 \\
\hline Dipropylamine & $142-84-7$ & 101.19 & 1.67 & & & & & & & 4 \\
\hline Phenol, 2,4-dinitro- & $51-28-5$ & & 1.67 & & & & & & & 4 \\
\hline Benzaldehyde, 2-hydroxy- & $90-02-8$ & & 1.66 & $4.90 \mathrm{E}+03$ & $\begin{array}{l}\text { Oryzias latipes } \\
\text { Pymenhales }\end{array}$ & flow-through & 96 & 1.60 & & 1 \\
\hline Ethene, chlorotrifluoro- & 79-38-9 & & 1.65 & $8.04 \mathrm{E}+02$ & & & & & & 4 \\
\hline Ethane, 1-chloro-1,1-difluoro- & $75-68-3$ & 100.50 & $1.64-2.05$ & $1.90 \mathrm{E}+04$ & Poecilia reticulata & & 96 & 220.00 & & 1 \\
\hline Hydroperoxide, 1-methyl-1-phenylethyl- & $80-15-9$ & & 1.60 & $1.43 \mathrm{E}+05$ & Oncorhynchus mykiss & & 96 & 3.90 & & 1 \\
\hline
\end{tabular}


(Table S7. continued)

\begin{tabular}{|c|c|c|c|c|c|c|c|c|c|c|}
\hline Chemicals name & CAS Reg. no & $\begin{array}{c}\text { Molecular } \\
\text { weight } \\
\left(\mathrm{g} \mathrm{mol}^{1}\right)\end{array}$ & $\log \mathrm{K}_{\mathrm{ow}}$ & Solubility $\left(\mathrm{mg} \mathrm{L}^{-1}\right)$ & Species & Exposure method & $\begin{array}{c}\text { Duration } \\
\text { (h) }\end{array}$ & $\begin{array}{c}\text { Measured } \\
\mathrm{LC}_{50} \\
\left(\mathrm{mg} \mathrm{L}^{-1}\right)\end{array}$ & $\begin{array}{c}\text { Nominal } \\
\text { LC } \\
\left(\mathrm{mg} \mathrm{L}^{-1}\right) \\
\end{array}$ & Classification \\
\hline 1,3-Isobenzofurandione & $85-44-9$ & & 1.60 & $1.64 \mathrm{E}+04$ & Cyprinus carpio & semi-static & 48 & & $>500$ & 3 \\
\hline Ethene, ethoxy- & $109-92-2$ & & 1.60 & $7.80 \mathrm{E}+03$ & Leuciscus idus & & 96 & & 3158.00 & 1 \\
\hline Aniline, 4,4'-methylenebis- & $101-77-9$ & 198.30 & 1.59 & $1.25 \mathrm{E}+03$ & $\begin{array}{l}\text { Oryzias latipes } \\
\text { Brachydanio rerio }\end{array}$ & semi-static & $\begin{array}{l}96 \\
96\end{array}$ & $\begin{array}{r}32.00 \\
210.00\end{array}$ & & 1 \\
\hline Ethene, chloro- & $75-01-4$ & & 1.58 & $1.10 \mathrm{E}+03$ & $\begin{array}{l}\text { Lepomis macrochirus } \\
\text { Micopterus salmoides }\end{array}$ & & $\begin{array}{l}96 \\
96\end{array}$ & & $\begin{array}{l}1220.00 \\
1060.00\end{array}$ & 1 \\
\hline 2-Butoxyethyl acetate & $112-07-2$ & & 1.57 & $1.50 \mathrm{E}+04$ & $\begin{array}{l}\text { Oncorhynchus mykiss } \\
\text { Pimephales promelas }\end{array}$ & $\begin{array}{l}\text { static } \\
\text { static }\end{array}$ & $\begin{array}{l}96 \\
96\end{array}$ & $\begin{array}{l}20-40 \\
31.00\end{array}$ & & 1 \\
\hline \multirow{2}{*}{$\begin{array}{l}\text { tert-Amyl methyl ether } \\
\text { Ethanol, 2-(hexyloxy)- }\end{array}$} & 994-05-8 & & 1.55 & $1.10 \mathrm{E}+04$ & Oncorhynchus mykiss & & 96 & 580.00 & & 1 \\
\hline & $112-25-4$ & & 1.55 & $9.90 \mathrm{E}+03$ & Brachydanio rerio & static & 96 & & $>94,<215$ & 1 \\
\hline Aniline, 3-nitro- & $99-09-2$ & 138.14 & 1.54 & $1.14 \mathrm{E}+03$ & $\begin{array}{l}\text { Oryzias ladipes } \\
\text { Oryzias ladipes }\end{array}$ & $\begin{array}{l}\text { static } \\
\text { semi-static }\end{array}$ & $\begin{array}{l}48 \\
96\end{array}$ & & $\begin{array}{l}96.00 \\
67.00\end{array}$ & 1 \\
\hline \multirow{2}{*}{$\begin{array}{l}\text { Pyridine, 2-vinyl- } \\
\text { Carbonochloridic acid, 1-methylpropyl ester } \\
\text { 1-Propanol, butoxy- }\end{array}$} & $\begin{array}{c}100-69-6 \\
17462-58-7\end{array}$ & 136.58 & $\begin{array}{l}1.54 \\
1.54\end{array}$ & $\begin{array}{l}2.67 \mathrm{E}+04 \\
4.22 \mathrm{E}+03\end{array}$ & $\begin{array}{l}\text { Oryzias latipes } \\
\text { Danio rerio }\end{array}$ & $\begin{array}{l}\text { semi-static } \\
\text { static }\end{array}$ & $\begin{array}{l}96 \\
96\end{array}$ & & $\begin{array}{l}6.50 \\
46.40\end{array}$ & $\begin{array}{l}1 \\
1\end{array}$ \\
\hline & $29387-86-8$ & 1500.00 & 1.52 & $5.50 \mathrm{E}+04$ & Poecilia reticulata & $\begin{array}{l}\text { static } \\
\text { stic }\end{array}$ & 96 & & $560 .-1000$ & 1 \\
\hline 2-Propanol, 1-phenoxy- & 770-35-4 & 152.19 & 1.52 & $1.00 \mathrm{E}+04$ & $\begin{array}{l}\text { Leuciscus idus } \\
\text { Pimephales promelas }\end{array}$ & $\begin{array}{l}\text { static } \\
\text { static }\end{array}$ & $\begin{array}{l}96 \\
96\end{array}$ & & $\begin{array}{c}215-464 \\
280.00\end{array}$ & 1 \\
\hline $\begin{array}{l}\text { Propanenitrile, 3-(trichlorosilyl)- } \\
\text { 1,3-Pentadiene }\end{array}$ & $\begin{array}{l}1071-22-3 \\
504-60-9\end{array}$ & 68.00 & $\begin{array}{l}1.52 \\
1.50\end{array}$ & $\begin{array}{l}1.37 \mathrm{E}+03 \\
6.90 \mathrm{E}+02\end{array}$ & Pimephales promelas & static & 96 & 139.90 & & $\begin{array}{l}4 \\
1\end{array}$ \\
\hline Propylene glycol phenyl ether (beta isomer - primary alcohol) & $4169-04-4$ & 0.00 & 1.50 & $1.00 \mathrm{E}+04$ & $\begin{array}{l}\text { Leuciscus idus } \\
\text { Pimephales promelas }\end{array}$ & $\begin{array}{l}\text { static } \\
\text { static }\end{array}$ & $\begin{array}{l}96 \\
96\end{array}$ & 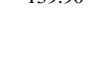 & $\begin{array}{l}215-464 \\
280.00\end{array}$ & 1 \\
\hline 1,4-Cyclohexanedimethanol & $105-08-8$ & & 1.49 & $9.20 \mathrm{E}+05$ & $\begin{array}{l}\text { Oryzias latipes } \\
\text { Pimephales promelas }\end{array}$ & $\begin{array}{l}\text { static } \\
\text { static }\end{array}$ & $\begin{array}{l}96 \\
96 \\
48\end{array}$ & & $\begin{array}{l}>100 \\
>120 \\
16-62\end{array}$ & 3 \\
\hline \multirow[t]{2}{*}{ Benzaldehyde } & $100-52-7$ & 106.12 & 1.48 & $6.55 \mathrm{E}+03$ & $\begin{array}{l}\text { Leuciscus idus } \\
\text { P.promelas } \\
\text { O. mykiss }\end{array}$ & $\begin{array}{l}\text { flow-through } \\
\text { flow-through }\end{array}$ & $\begin{array}{l}48 \\
96 \\
96\end{array}$ & $\begin{array}{l}12.40 \\
11.20\end{array}$ & $16-62$ & 1 \\
\hline & & & & & L. macrochirus & $\begin{array}{l}\text { flow-through } \\
\text { for }\end{array}$ & 96 & 1.07 & & \\
\hline Ethane, pentafluoro- & $354-33-6$ & & 1.48 & $4.32 \mathrm{E}-01-1.07 \mathrm{E}+00$ & & & & & & 4 \\
\hline \multirow[t]{2}{*}{ Phenol } & $108-95-2$ & & 1.47 & $8.40 \mathrm{E}+04$ & $\begin{array}{l}\text { Lepomis macrochirus } \\
\text { Pimephales promelas } \\
\text { Oncorhynchus mykiss }\end{array}$ & $\begin{array}{l}\text { Static } \\
\text { flow-through } \\
\text { semi-static }\end{array}$ & $\begin{array}{l}96 \\
96 \\
96\end{array}$ & $\begin{array}{c}17.00 \\
24.9-67.5 \\
10.50\end{array}$ & & 1 \\
\hline & & & & & Brachydanio rerio & flow-through & 96 & 29.00 & & \\
\hline \multirow[b]{2}{*}{ Triethylamine- } & $10025-78-2$ & & 1.46 & & & & & & & 4 \\
\hline & $121-44-8$ & 101.19 & 1.45 & & $\begin{array}{l}\text { Oncorhynchus mykiss } \\
\text { Micropterus salmoides }\end{array}$ & $\begin{array}{l}\text { flow-through } \\
\text { static }\end{array}$ & $\begin{array}{l}96 \\
96\end{array}$ & $\begin{array}{l}36.00 \\
66.00\end{array}$ & & 1 \\
\hline Ethane, 1,2-dichloro- & $107-06-2$ & & 1.45 & $8.49 \mathrm{E}+03-9.00 \mathrm{E}+03$ & $\begin{array}{l}\text { Limanda limanda } \\
\text { Pimephales promelas }\end{array}$ & $\begin{array}{l}\text { flow-through } \\
\text { flow-through }\end{array}$ & $\begin{array}{l}96 \\
96\end{array}$ & $\begin{array}{l}115.00 \\
116.00\end{array}$ & & 1 \\
\hline Ethane, chloro- & $75-00-3$ & & 1.43 & $5.74 \mathrm{E}+03$ & $\begin{array}{l}\text { Lepomis macrochirus } \\
\text { Micropterus salmoides }\end{array}$ & $\begin{array}{l}\text { static } \\
\text { static }\end{array}$ & $\begin{array}{l}96 \\
96\end{array}$ & 2250.00 & $>2000$ & 1 \\
\hline \multirow[t]{2}{*}{ Aniline, 2-methyl- } & $95-53-4$ & & 1.40 & $1.50 \mathrm{E}+04$ & $\begin{array}{l}\text { Oryzias latipes } \\
\text { Leuciscus idus }\end{array}$ & $\begin{array}{l}\text { static } \\
\text { static }\end{array}$ & $\begin{array}{l}96 \\
96\end{array}$ & & $\begin{array}{l}151.00 \\
82.50\end{array}$ & 1 \\
\hline & & & & & Leuciscus idus & & 96 & & 26.00 & \\
\hline \multirow[t]{2}{*}{ 2-Propanamine, N-(1-methylethyl)- } & $108-18-9$ & 101.19 & 1.40 & & $\begin{array}{l}\text { Oncorhynchus mykiss } \\
\text { Gasterosteus aculeatus }\end{array}$ & & $\begin{array}{l}96 \\
96\end{array}$ & 79800 & 196.00 & 1 \\
\hline & & & & & Pimephales promelas & & 96 & 170.00 & 40.00 & \\
\hline \multirow{2}{*}{$\begin{array}{l}\mathrm{m} \text {-Toluidine } \\
\text { Hydroperoxide, 1,1-dimethylpropyl- }\end{array}$} & $108-44-1$ & 107.16 & 1.40 & $1.00 \mathrm{E}+04$ & Oryzias latipes & semi-static & 96 & & 34.00 & 1 \\
\hline & $3425-61-4$ & & 1.40 & & Pimephales promelas & static & 96 & & 77.00 & 4 \\
\hline \multirow[t]{2}{*}{ Butyric anhydride } & $106-31-0$ & & 1.39 & $4.56 \mathrm{E}+03$ & $\begin{array}{l}\text { Pimephales promelas } \\
\text { Pimephales promelas }\end{array}$ & static & 96 & & 51.80 & 1 \\
\hline & 106-49-0 & & 1.39 & $7.40 \mathrm{E}+03$ & $\begin{array}{l}\text { Oryzias latipes } \\
\text { Danio rerio }\end{array}$ & $\begin{array}{l}\text { static-renewal } \\
\text { flow-through }\end{array}$ & $\begin{array}{l}48 \\
96\end{array}$ & 11500 & 90.00 & 1 \\
\hline \multirow{2}{*}{ Pentanoic acid } & $109-52-4$ & 102.13 & 139 & $2,40 \mathrm{E}+04$ & Pimephales promelas & $\begin{array}{l}\text { flow-through } \\
\text { static }\end{array}$ & 96 & 110.00 & 77.00 & 1 \\
\hline & $109-52-4$ & 102.13 & 1.39 & $2.40 \mathrm{E}+04$ & Pimephales promelas & & 96 & & 39.00 & 1 \\
\hline 1,3,5-Triazine-2,4-diamine, 6-phenyl- & $91-76-9$ & & 1.38 & $3.20 \mathrm{E}+02$ & $\begin{array}{l}\text { Oryzias latipes } \\
\text { Leuciscus idus }\end{array}$ & $\begin{array}{l}\text { semi-static } \\
\text { static }\end{array}$ & $\begin{array}{l}96 \\
48\end{array}$ & $\begin{array}{l}>100 \\
99.00\end{array}$ & & 1 \\
\hline Pentanal & $110-62-3$ & & 1.38 & $1.17 \mathrm{E}+04$ & Oncorhynchus mykiss & & 96 & 27.90 & & 1 \\
\hline Benzoic acid, 4-hydroxy- & 99-96-7 & & 1.37 & $6.00 \mathrm{E}+03$ & $\begin{array}{l}\text { Pimephales promelas } \\
\text { Oryzias latipes }\end{array}$ & flow-through & $\begin{array}{l}96 \\
96\end{array}$ & $\begin{array}{l}12.40 \\
92.80\end{array}$ & & 1 \\
\hline 3-Penten-2-one, 4-methyl- (Mesityl oxide) & $141-79-7$ & 98.15 & 1.37 & $3.00 \mathrm{E}+04$ & $\begin{array}{l}\text { Oncorhynchus mykiss } \\
\text { Pimephales promelas }\end{array}$ & $\begin{array}{l}\text { static } \\
\text { static }\end{array}$ & $\begin{array}{l}96 \\
96\end{array}$ & & $\begin{array}{l}71.00 \\
86.00\end{array}$ & 1 \\
\hline
\end{tabular}


(Table S7. continued)

\begin{tabular}{|c|c|c|c|c|c|c|c|c|c|c|}
\hline Chemicals name & CAS Reg. no & $\begin{array}{c}\text { Molecular } \\
\text { weight } \\
\left(\mathrm{g} \mathrm{mol}^{-1}\right)\end{array}$ & $\log K_{\text {ow }}$ & Solubility $\left(\mathrm{mg} \mathrm{L}^{-1}\right)$ & Species & Exposure method & $\begin{array}{l}\text { Duration } \\
\text { (h) }\end{array}$ & $\begin{array}{c}\text { Measured } \\
\mathrm{LC}_{50} \\
\left(\mathrm{mg} \mathrm{L}^{-1}\right) \\
\end{array}$ & $\begin{array}{c}\text { Nominal } \\
\mathrm{LC}_{50} \\
\left(\mathrm{mg} \mathrm{L}^{-1}\right) \\
\end{array}$ & Classification \\
\hline \multirow[t]{3}{*}{ Vanillin } & $121-33-5$ & 152.14 & 1.35 & $1.00 \mathrm{E}+04$ & $\begin{array}{l}\text { Pimephales promelas } \\
\text { Pimephales promelas }\end{array}$ & $\begin{array}{l}\text { flow-through } \\
\text { semi-static }\end{array}$ & $\begin{array}{l}96 \\
96\end{array}$ & $\begin{array}{l}123.00 \\
57.00\end{array}$ & & 1 \\
\hline & & & & & $\begin{array}{l}\text { Leuciscus idus } \\
\text { L }\end{array}$ & & 48 & $>10000$ & & \\
\hline & & & & & Poecilia reticulata & & 48 & 9160.00 & & \\
\hline \multirow{3}{*}{ Trichloroacetic acid } & 76-03-9 & & 1.33 & & Oryzias latipes & & 48 & 277.00 & & 1 \\
\hline & & & & & Alburnus alburnus & & 96 & 9300.00 & & \\
\hline & & & & & Pimephales promelas & & 96 & 2000.00 & & \\
\hline Butanal, 3-methyl- & $590-86-3$ & & 1.32 & $2.00 \mathrm{E}+04$ & Pimephales promelas & flow-through & 96 & 3.25 & & 1 \\
\hline Ethanol, 2-(2-butoxyethoxy)-, acetate & $124-17-4$ & 204.27 & 1.30 & & Pimephales promelas & static & 96 & & 110.00 & 1 \\
\hline $2 \mathrm{H}$-Pyran, 3,4-dihydro-2-methoxy- & $4454-05-1$ & 114.14 & 1.30 & $1.60 \mathrm{E}+04$ & Oryzias latipes & static & 96 & & 730.00 & 1 \\
\hline Aniline, 4-ethoxy- & $156-43-4$ & 137.18 & 1.28 & $2.10 \mathrm{E}+04$ & $\begin{array}{l}\text { Oryzias latipes } \\
\text { Orvizas lotipes }\end{array}$ & static & 48 & 234.00 & & 1 \\
\hline Fthanethiol & $75-08-1$ & & 27 & $156 \mathrm{~F}+04$ & Oncorhynchus mykiss & stantc & $\begin{array}{l}48 \\
96\end{array}$ & $\begin{array}{l}100.00 \\
2.40\end{array}$ & & 1 \\
\hline Ethanethiol & $75-08-1$ & & 1.27 & $1.56 \mathrm{E}+04$ & Oryzias latipes & & 96 & 2.23 & & 1 \\
\hline \multirow[t]{2}{*}{ Ethanol, 2-[2-(hexyloxy)ethoxy]- } & $112-59-4$ & 190.29 & 1.27 & $1.70 \mathrm{E}+04$ & & & & & & 4 \\
\hline & & & & & Pimephales promelas & & 96 & 193.00 & & \\
\hline \multirow[t]{3}{*}{ Methane, dichloro- } & $75-09-2$ & & 1.25 & $1.32 \mathrm{E}+04$ & Pimephales promelas & & 96 & 502.00 & & 1 \\
\hline & & & & & Pimephales promelas & & 96 & 330.00 & & 1 \\
\hline & & & & & Fundulus heteroclitus & & 48 & 97.00 & & \\
\hline Propanoic acid, 2-methyl-, anhydride & $97-72-3$ & & 1.24 & $1.60 \mathrm{E}+04$ & $\begin{array}{l}\text { Pimephales promelas } \\
\text { Pimephales promelas }\end{array}$ & $\begin{array}{l}\text { static } \\
\text { static }\end{array}$ & $\begin{array}{l}96 \\
96\end{array}$ & $\begin{array}{l}77.00 \\
51.80\end{array}$ & & 1 \\
\hline Propyl acetate & $109-60-4$ & & 1.24 & $2.00 \mathrm{E}+04$ & Oryzias latipes & & 96 & & $>100$ & 3 \\
\hline Ethene, 1,1-difluoro- & $75-38-7$ & 64.00 & 1.24 & $2.54 \mathrm{E}+02$ & & & & & & 4 \\
\hline \multirow[t]{2}{*}{ Propyl chloroformate } & $109-61-5$ & 122.55 & 1.22 & $1.09 \mathrm{E}+04$ & Danio rerio & static & 96 & & 3.16 & 1 \\
\hline & & & & & Leuciscus idus & flow-through & 96 & $22>\mathrm{LC}_{50}$ & & \\
\hline \multirow{3}{*}{ Ethyl acrylate } & $140-88-5$ & & 1.18 & $1.50 \mathrm{E}+04$ & Pimephales promelas & flow-through & 96 & & 2.50 & 1 \\
\hline & & & & & Carassius auratus & flow-through & 72 & & 5.00 & \\
\hline & & & & & Species not indicated & flow-through & 72 & & 5.00 & \\
\hline Aniline, 2-methoxy- & 90-04-0 & & 1.18 & & & & & & & 4 \\
\hline \multirow[t]{2}{*}{ Butanoic acid, 2-methyl- } & $116-53-0$ & 102.13 & 1.18 & & & & & & & 4 \\
\hline & & & & & $\begin{array}{l}\text { Leuciscus idus } \\
\text { melanotus }\end{array}$ & static & 96 & $>922$ & & \\
\hline \multirow[t]{2}{*}{ Terephthalic acid } & $100-21-0$ & & 1.16 & $1.90 \mathrm{E}+04$ & $\begin{array}{l}\text { melanotus } \\
\text { Salmo gairdneri }\end{array}$ & semi-static & 96 & & $798-1640$ & 1 \\
\hline & & & & & Brachydanio rerio & & 96 & & $>500$ & \\
\hline \multirow[t]{2}{*}{ Ethanol, 2-phenoxy- } & $122-99-6$ & 138.17 & 1.16 & $2.89 \mathrm{E}+04$ & Pimephales promelas & flow-through & 96 & 344.00 & & 1 \\
\hline & & & & & Oryzias latipes & static & 96 & & $>100$ & \\
\hline \multirow[t]{3}{*}{ Acetamide, N-phenyl- } & $103-84-4$ & & 1.16 & $4.00 \mathrm{E}+03$ & Lepomis macrochirus & static & 96 & & 100.00 & 1 \\
\hline & & & & & Leuciscus idus & static & 48 & & 200.00 & \\
\hline & & & & & Carassius auratus & static & 48 & & 460.00 & \\
\hline \multirow{3}{*}{ Methyl isobutyl ketone } & $108-10-1$ & 100.16 & 1.16 & $1.70 \mathrm{E}+04-2.04 \mathrm{E}+04$ & Pimephales promelas & static & 96 & & 780.00 & 1 \\
\hline & & & & & $\begin{array}{l}\text { Leuciscus idus } \\
\text { melanotus }\end{array}$ & static & 48 & & $675-750$ & \\
\hline & & & & & $\begin{array}{l}\text { melanotus } \\
\text { Oryzias latipes }\end{array}$ & semi-static & 96 & & 100.00 & \\
\hline \multirow{2}{*}{ Phthalimide } & $85-41-6$ & & 1.15 & $3.70 \mathrm{E}+02$ & Salmo gairdneri & static & 96 & & 51.00 & 1 \\
\hline & & & & & Lepomis macrochirus & static & 96 & & 53.00 & \\
\hline 2-Propanol, 1-butoxy- & 5131-66-8 & & 1.15 & $5.50 \mathrm{E}+04$ & Poecilia reticulata & Static & 96 & & $560-1000$ & 1 \\
\hline 2,5,7,10-Tetraoxa-6-silaundecane, 6-ethenyl-6-(2-methoxyethoxy)- & $1067-53-4$ & & 1.14 & $5.00 \mathrm{E}+03-1.00 \mathrm{E}+04$ & & & & & & 4 \\
\hline 2-(Dimethylamino)ethyl methacrylate & $2867-47-2$ & & 1.13 & $1.06 \mathrm{E}+05$ & Oryzias latipes & semi-static & 96 & 19.10 & & 1 \\
\hline 2-Propanol, 1-(2-butoxy-1-methylethoxy)- & 29911-28-2 & 190.29 & 1.13 & $4.50 \mathrm{E}+04$ & Poecilia reticulata & static & 96 & & 841.00 & 1 \\
\hline \multirow{2}{*}{$\begin{array}{l}\text { Ethene } \\
\text { Etropol, 1-(2-Dutoxy-1-metnyletnoxy)- }\end{array}$} & 74-85-1 & & 1.13 & $1.31 \mathrm{E}+02$ & & & & & & 4 \\
\hline & & & & & Leuciscus idus & & 48 & & 2140.00 & \\
\hline \multirow[t]{2}{*}{ Phosphoric acid, triethyl ester } & $78-40-0$ & & 1.11 & miscible & $\begin{array}{l}\text { Pimephales } \\
\text { promephales }\end{array}$ & & 96 & & $>1000$ & 1 \\
\hline & & & & & Oryzias latipes & & 48 & & $>500$ & \\
\hline Benzenemethanol & $100-51-6$ & & 1.10 & $4.00 \mathrm{E}+04$ & Pimephales promelas & static & 96 & & 460.00 & 1 \\
\hline Propanenitrile, 2,2'-azobis[2-methyl- & $78-67-1$ & & 1.10 & $3.50 \mathrm{E}+02$ & $\begin{array}{l}\text { Leuciscus idus } \\
\text { Oryzias latipes }\end{array}$ & $\begin{array}{l}\text { static } \\
\text { semi-stati }\end{array}$ & $\begin{array}{l}96 \\
96\end{array}$ & $>10$ & 646.00 & 3 \\
\hline 1-Methylethyl carbonochloridiate & $108-23-6$ & 122.55 & 1.04 & $1.26 \mathrm{E}+04$ & Danio rerio & semi-static & 96 & 8.20 & & 1 \\
\hline 1-methylethyl acetate & $108-21-4$ & & 1.02 & $3.09 \mathrm{E}+04$ & Pimephales promelas & static & 96 & & 390.25 & 1 \\
\hline
\end{tabular}




\section{(Table S7. continued)}

\begin{tabular}{|c|c|c|c|c|c|c|c|c|c|c|}
\hline Chemicals name & CAS Reg. no & $\begin{array}{l}\text { Molecular } \\
\text { weight } \\
\left(\mathrm{g} \mathrm{mol}^{-1}\right)\end{array}$ & $\log K_{\text {ow }}$ & Solubility $\left(\mathrm{mg} \mathrm{L}^{-1}\right)$ & Species & Exposure method & $\begin{array}{l}\text { Duration } \\
\text { (h) }\end{array}$ & $\begin{array}{c}\text { Measured } \\
\mathrm{LC}_{50} \\
\left(\mathrm{mg} \mathrm{L}^{-1}\right) \\
\end{array}$ & $\begin{array}{c}\begin{array}{c}\text { Nominal } \\
\mathrm{LC}_{50} \\
\left(\mathrm{mg} \mathrm{L}^{-1}\right)\end{array} \\
\end{array}$ & Classification \\
\hline Aniline, 3-methoxy- & $536-90-3$ & 123.00 & 1.01 & $2.05 \mathrm{E}+04$ & Oryzias latipes & Semi-static & 96 & & 240.00 & 1 \\
\hline Pyrocatechol & $120-80-9$ & & 1.01 & $4.49 \mathrm{E}+05$ & freshwater fish & & 96 & 8.90 & & 1 \\
\hline Cyclohexylamine, 3-aminomethyl-3,5,5-trimethyl- & 2855-13-2 & 170.30 & 0.99 & miscible & Leuciscus idus & semi-static & 96 & 110.00 & & 1 \\
\hline 1-Butanamine & $109-73-9$ & 73.14 & 0.97 & & Pimephales promelas & & 96 & 268.00 & & 1 \\
\hline Diethyl malonate & $105-53-3$ & & 0.96 & $2.00 \mathrm{E}+04$ & $\begin{array}{l}\text { Pimephales promelas } \\
\text { Pimephales promelas }\end{array}$ & $\begin{array}{l}\text { flow through } \\
\text { flow through }\end{array}$ & $\begin{array}{l}96 \\
96\end{array}$ & $\begin{array}{l}12.00 \\
15.00\end{array}$ & & 1 \\
\hline Glycidyl methacrylate & $106-91-2$ & & 0.96 & $5.00 \mathrm{E}+04$ & Oryzias latipes & semi-static & 96 & 2.80 & & 1 \\
\hline \multirow[t]{4}{*}{ 1,2,4-Benzenetricarboxylic acid } & $528-44-9$ & & 0.95 & $2.10 \mathrm{E}+04$ & $\begin{array}{l}\text { Leuciscus idus } \\
\text { melanotus }\end{array}$ & static & 96 & & $\begin{array}{l}\text { not } \\
\text { determined }\end{array}$ & 3 \\
\hline & & & & & Pimephales promelas & & 96 & 672.00 & & \\
\hline & & & & & Pimephales promelas & & 96 & 706.00 & & \\
\hline & & & & & Pimephales promelas & & 96 & 980.00 & & \\
\hline \multirow[t]{4}{*}{ Methyl t-butyl ether } & $1634-04-4$ & 88.15 & 0.94 & & Oncorhynchus mykiss & & 96 & 887.00 & & 1 \\
\hline & & & & & Lepomis macrochirus & & 96 & 1054.00 & & \\
\hline & & & & & Gasterosteus aculeatus & & 96 & 929.00 & & \\
\hline & & & & & $\begin{array}{l}\text { Cyprinodon variegatus } \\
\text { Pimonalos }\end{array}$ & & 96 & 1358.00 & & \\
\hline \multirow[t]{2}{*}{ Propanoic acid, 2-methyl- } & $79-31-2$ & & 0.94 & $1.67 \mathrm{E}+02$ & $\begin{array}{l}\text { Pimephales promelas } \\
\text { Pimenhales promelas }\end{array}$ & static & 96 & & $\begin{array}{l}77.00 \\
5180\end{array}$ & 1 \\
\hline & & & & & $\begin{array}{l}\text { Pimephales promelas } \\
\text { zebrafish }\end{array}$ & static & 96 & & 51.80 & \\
\hline Propane, 2-nitro- & $79-46-9$ & & 0.93 & $1.70 \mathrm{E}+04$ & $\begin{array}{l}\text { zebratish } \\
\text { Pimephales promelas }\end{array}$ & & 48 & $\begin{array}{l}620.00 \\
515.00\end{array}$ & & 1 \\
\hline \multirow{3}{*}{$\begin{array}{l}\text { Methacrylic acid } \\
\text { Dimethyl sulfide } \\
\text { 2-Buten-1-ol, 3-methyl- }\end{array}$} & $79-41-4$ & & 0.93 & $8.90 \mathrm{E}+04$ & Oncorhynchus mykiss & & 96 & 85.00 & & 4 \\
\hline & $75-18-3$ & & 0.92 & $2.00 \mathrm{E}+04$ & Oncorhynchus mykiss & semistatic & 96 & 213.00 & & 1 \\
\hline & $556-82-1$ & & 0.91 & $1.70 \mathrm{E}+05$ & Leuciscus idus & static & 96 & 46.00 & & 1 \\
\hline Methane, chloro- & $74-87-3$ & 50.49 & 0.91 & $4.80 \mathrm{E}+03-5.32 \mathrm{E}+03$ & $\begin{array}{l}\text { Lepomis macrochirus } \\
\text { Menidia beryllina }\end{array}$ & $\begin{array}{l}\text { static } \\
\text { static }\end{array}$ & $\begin{array}{l}96 \\
96\end{array}$ & & $\begin{array}{l}550.00 \\
270.00\end{array}$ & 1 \\
\hline \multirow{2}{*}{ Propanoic acid, 3-mercapto-, methyl ester } & 2935-90-2 & & 0.90 & $2.10 \mathrm{E}+04$ & $\begin{array}{l}\text { Mencida beryllina } \\
\text { Oncorhynchus mykss }\end{array}$ & flow-through & 96 & 1.70 & & 1 \\
\hline & & & & & Oncorhynchus mykiss & flow-through & 96 & 10.60 & & \\
\hline \multirow{2}{*}{ Aniline } & $62-53-3$ & & 0.90 & $3.50 \mathrm{E}+04$ & Oncorhynchus mykiss & flow-through & 96 & 36.20 & & 1 \\
\hline & & & & & Brachydanio rerio & semi-static & 96 & 57.50 & & \\
\hline Phenol, 2,4,6-trinitro- & 88-89-1 & & 0.89 & $1.18 \mathrm{E}+04$ & Oncorhynchus mykiss & semi-static & 96 & & 110.00 & 1 \\
\hline Propanoyl chloride, 2,2-dimethyl- & $3282-30-2$ & 120.58 & 0.89 & $3.48 \mathrm{E}+04$ & Brachydanio rerio & static & 96 & 287.00 & & 1 \\
\hline \multirow{2}{*}{$\mathrm{n}$-Butanal } & $123-72-8$ & 72.11 & 0.88 & $1.18 \mathrm{E}+02$ & Pimephales promelas & static renewal & 96 & 25.80 & & 1 \\
\hline & & 12.11 & & & Leuciscus idus & static & 96 & 57.00 & & 1 \\
\hline \multirow[t]{2}{*}{ 1-Butanol } & $71-36-3$ & & 0.88 & $7.70 \mathrm{E}+04$ & $\begin{array}{l}\text { Pimephales promelas } \\
\text { Alburus alburnus }\end{array}$ & $\begin{array}{l}\text { static } \\
\text { static }\end{array}$ & 96 & 1376.00 & 1730.00 & 1 \\
\hline & & & & & $\begin{array}{l}\text { Alburnus aburinus } \\
\text { Oryzias latipes }\end{array}$ & semi-static & 96 & $>100$ & 1700.00 & \\
\hline \multirow[t]{2}{*}{ Butanamide, N-(2-methylphenyl)-3-oxo- } & $93-68-5$ & & 0.85 & $3.00 \mathrm{E}+03$ & Brachydanio rerio & static & 96 & $>500$ & & 1 \\
\hline & & & & & Pimephales promelas & static & 96 & 316.20 & & \\
\hline $\mathrm{o}$-Toluenesulfonamide & $88-19-7$ & & 0.84 & $1.60 \mathrm{E}+03$ & Oryzias latipes & semi-static & 96 & & $>100$ & 3 \\
\hline D-Mannitol & $69-65-8$ & 171.23 & 0.84 & & & & & & & 4 \\
\hline Carbon dioxide & $124-38-9$ & 44.01 & 0.83 & & & & & & & 4 \\
\hline p-Toluenesulfonamide & $70-55-3$ & & 0.82 & $3.20 \mathrm{E}+03$ & Oryzias latipes & Semi-static & 96 & 435.00 & & 1 \\
\hline \multirow[t]{2}{*}{ Ethanol, 2-butoxy- } & $111-76-2$ & & 0.80 & $5.00 \mathrm{E}+01$ & & & & 100000 & & 4 \\
\hline & & & & & Pimephales promelas & flow-through & 96 & 29.50 & & \\
\hline \multirow[t]{2}{*}{ 1,3-Benzenediol } & $108-46-3$ & & 0.80 & $7.17 \mathrm{E}+05$ & Pimephales promelas & flow-through & 96 & 26.80 & & 1 \\
\hline & & & & & Leuciscus idus & static & 96 & & 34.70 & \\
\hline Butanoic acid & $107-92-6$ & & 0.79 & miscible & Oryzias latipes & static renewal & 48 & 90.00 & & 1 \\
\hline Propane, 1-nitro- & $108-03-2$ & & 0.79 & $1.40 \mathrm{E}+04$ & Oncorhychus mykiss & Flow-through & 96 & 227.00 & & 1 \\
\hline 1-Propanol, 2-methyl- & $78-83-1$ & 74.12 & 0.79 & $8.50 \mathrm{E}+04$ & Pimephales promelas & flow through & 96 & 1430.00 & & 1 \\
\hline Methanethiol & 74-93-1 & & 0.78 & $2.33 \mathrm{E}+01$ & & & & & & 4 \\
\hline Propanal, 2-methyl- & $78-84-2$ & 72.11 & 0.77 & $8.90 \mathrm{E}+04$ & $\begin{array}{l}\text { Pimephales promelas } \\
\text { Leeciscus idus }\end{array}$ & $\begin{array}{l}\text { semi-static } \\
\text { static }\end{array}$ & 96 & 23.00 & 86.00 & 1 \\
\hline Hexane-1,6-diol & $629-11-8$ & 118.18 & 0.76 & miscible & Leuciscus idus & & 96 & & $460-1000$ & 1 \\
\hline Acetic acid, cyano- & $372-09-8$ & & 0.76 & $8.90 \mathrm{E}+05-1.00 \mathrm{E}+06$ & Leuciscus idus & static & 96 & & $>46.4-100$ & 3 \\
\hline $\begin{array}{l}\text { Ethyl cyanoacetate } \\
\text { Fthane 11 filloro }\end{array}$ & $105-56-6$ & & 0.76 & $2.00 \mathrm{E}+04$ & 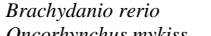 & flow through & 96 & 59.00 & & 1 \\
\hline Ethane, 1,1-difluoro- & $75-37-6$ & 66.10 & 0.75 & $2.67 \mathrm{E}+03$ & Oncorhynchus mykiss & semi-static & 96 & 450.00 & & 1 \\
\hline Methyl acrylate & $96-33-3$ & & 0.74 & $5.20 \mathrm{E}+04$ & $\begin{array}{l}\text { Oncorhynchus mykiss } \\
\text { Cyprinodon variegatus }\end{array}$ & $\begin{array}{l}\text { flow-through } \\
\text { flow-through }\end{array}$ & $\begin{array}{l}96 \\
96\end{array}$ & $\begin{array}{l}3.40 \\
1.10\end{array}$ & & 1 \\
\hline Silanetriol, ethyl-, triacetate & 17689-77-9 & 234.28 & 0.74 & $4.16 \mathrm{E}+04$ & Brachydanio rerio & semi-static & $\begin{array}{l}96 \\
96\end{array}$ & 251.00 & $>46-<68$ & $\begin{array}{l}1 \\
1\end{array}$ \\
\hline 2-Butanamine & 13952-84-6 & 73.14 & 0.74 & & Lenciscus idus & & & & $>46-<68$ & \\
\hline
\end{tabular}


(Table S7. continued)

\begin{tabular}{|c|c|c|c|c|c|c|c|c|c|c|}
\hline Chemicals name & CAS Reg. no & $\begin{array}{c}\text { Molecular } \\
\text { weight } \\
\left(\mathrm{g} \mathrm{mol}^{-1}\right)\end{array}$ & $\log \mathrm{K}_{\mathrm{ow}}$ & Solubility $\left(\mathrm{mg} \mathrm{L}^{-1}\right)$ & Species & Exposure method & $\begin{array}{l}\text { Duration } \\
\text { (h) }\end{array}$ & $\begin{array}{c}\text { Measured } \\
\mathrm{LC}_{50} \\
\left(\mathrm{mg} \mathrm{L}^{-1}\right) \\
\end{array}$ & $\begin{array}{c}\text { Nominal } \\
\mathrm{LC}_{50} \\
\left(\mathrm{mg} \mathrm{L}^{-1}\right) \\
\end{array}$ & Classification \\
\hline $\begin{array}{l}\text { Triethyl phosphite } \\
\text { Vinyl acetate }\end{array}$ & $\begin{array}{l}122-52-1 \\
108-05-4\end{array}$ & $\begin{array}{c}166.16 \\
86.09\end{array}$ & $\begin{array}{l}0.74 \\
0.73\end{array}$ & $1.50 \mathrm{E}+04$ & Brachydanio rerio & static & 96 & & 251.60 & $\begin{array}{l}1 \\
4\end{array}$ \\
\hline Ethyl acetate & $141-78-6$ & & 0.73 & $8.30 \mathrm{E}+04$ & $\begin{array}{l}\text { Fathead minnows } \\
\text { Fathead minnows }\end{array}$ & $\begin{array}{l}\text { flow-through } \\
\text { flow-through }\end{array}$ & $\begin{array}{l}96 \\
96\end{array}$ & $\begin{array}{l}230.00 \\
>75.6\end{array}$ & & 1 \\
\hline $\begin{array}{l}\text { Ethylamine, N,N-dimethyl- } \\
\text { Ethylamine, N,N-dimethyl- }\end{array}$ & $\begin{array}{l}598-56-1 \\
598-56-1\end{array}$ & $\begin{array}{l}73.14 \\
73.14\end{array}$ & $\begin{array}{l}0.70 \\
0.70\end{array}$ & & $\begin{array}{l}\text { Leuciscus idus } \\
\text { Leuciscus idus }\end{array}$ & $\begin{array}{l}\text { static } \\
\text { static }\end{array}$ & $\begin{array}{l}96 \\
96\end{array}$ & & $\begin{array}{l}>100 \\
38.30\end{array}$ & 1 \\
\hline Acetoacetanilide & $102-01-2$ & & 0.70 & $8.37 \mathrm{E}+00-1.00 \mathrm{E}+01$ & Brachydanio rerio & static & 96 & & $>242$ & 3 \\
\hline $\begin{array}{l}\text { Hydroperoxide, tert-butyl- } \\
\text { Mucochloric acid }\end{array}$ & $\begin{array}{l}75-91-2 \\
87-56-9\end{array}$ & 90.12 & $\begin{array}{l}0.70 \\
0.70\end{array}$ & $\begin{array}{l}2.20 \mathrm{E}+01 \\
2.70 \mathrm{E}+04\end{array}$ & Leuciscus idus & static & 96 & & 123.00 & $\begin{array}{l}4 \\
1\end{array}$ \\
\hline $\begin{array}{l}\text { 1-Propanaminium, N-(carboxymethyl)-N,N-dimethyl-3-[(1- } \\
\text { oxododecyl)amino]-, }\end{array}$ & $4292-10-8$ & 343.53 & 0.69 & $1.76 \mathrm{E}+03$ & & & & & & 4 \\
\hline $\begin{array}{l}\text { 2-(Dimethylamino)ethyl acrylate } \\
\text { 2-(Dimethylamino)ethyl acrylate }\end{array}$ & $\begin{array}{l}2439-35-2 \\
2439-35-2\end{array}$ & & $\begin{array}{l}0.68 \\
0.68\end{array}$ & $\begin{array}{l}2.40 \mathrm{E}+04 \\
2.40 \mathrm{E}+04\end{array}$ & $\begin{array}{l}\text { Oryzias latipes } \\
\text { Leucisuc idus }\end{array}$ & & $\begin{array}{l}96 \\
96\end{array}$ & & $\begin{array}{c}8.49 \\
16.00\end{array}$ & 1 \\
\hline 2-Oxepanone & 502-44-3 & & 0.68 & miscible & $\begin{array}{l}\text { Poecilia reticulata } \\
\text { Pimephales promelas }\end{array}$ & $\begin{array}{l}\text { static } \\
\text { static }\end{array}$ & 96 & 295.00 & 32000 & 1 \\
\hline 2-Propenenitrile, 2-methyl- & $126-98-7$ & & 0.68 & $2.90 \mathrm{E}+04$ & 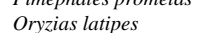 & semistatic & 96 & & $>100$ & 3 \\
\hline Butane, 1,2-epoxy- & $106-88-7$ & & 0.68 & $5.90 \mathrm{E}+04$ & Leuciscus idus & static & 96 & & $100-215$ & 1 \\
\hline Diaminotoluene & $25376-45-8$ & & 0.66 & $1.39 \mathrm{E}+04$ & & & & & & 4 \\
\hline Toluene-3,4-diamine & 496-72-0 & & 0.66 & $1.39 \mathrm{E}+04$ & & & & & & 4 \\
\hline $\begin{array}{l}\text { 3-Buten-2-ol, 2-methyl- } \\
\text { 2-Butanone, oxime }\end{array}$ & $\begin{array}{l}115-18-4 \\
96-29-7\end{array}$ & & $\begin{array}{l}0.66 \\
0.65\end{array}$ & $1.90 \mathrm{E}+05$ & Leuciscus idus & static & 96 & & $2150<<4640$ & 1 \\
\hline $\begin{array}{l}\text { 2-Butanone, oxime } \\
\text { Benzenesulfonic acid, 2,2'-(1,2-ethenediyl)bis[5-[[4-[bis(2- }\end{array}$ & $\begin{array}{c}96-29-7 \\
4404-43-7\end{array}$ & 960.95 & $\begin{array}{l}0.65 \\
0.65\end{array}$ & $1.10 \mathrm{E}+05$ & & static & 96 & & $>180$ & $\begin{array}{l}4 \\
3\end{array}$ \\
\hline $\begin{array}{l}\text { hydroxyethyl)amino]-6-(phenylamino)-1,3,5-triazin-2-yl]amino]- } \\
\text { Carbonochloridic acid, ethyl ester }\end{array}$ & $\begin{array}{l}4404-43-7 \\
541-41-3\end{array}$ & $\begin{array}{l}900.93 \\
108.53\end{array}$ & 0.63 & $3.21 \mathrm{E}+04$ & Pimephales promelas & stancic & & & & 4 \\
\hline Butan-2-ol & $78-92-2$ & & 0.61 & $4.50 \mathrm{E}+05$ & Pimephales Promelas & flow through & 96 & 3670.00 & & 1 \\
\hline Propanal & $123-38-6$ & 58.08 & 0.59 & $2.20 \mathrm{E}+05$ & Pimephales promelas & static & 96 & & 14.00 & 1 \\
\hline Silane, dimethoxydimethyl- & $1112-39-6$ & & 0.59 & $6.80 \mathrm{E}+03$ & & & & & & 4 \\
\hline Phthalonitrile & $91-15-6$ & & 0.58 & $5.60 \mathrm{E}+02$ & $\begin{array}{l}\text { Oryzias latipes } \\
\text { Pimephales promelas }\end{array}$ & $\begin{array}{l}\text { semi-static } \\
\text { flow-through }\end{array}$ & $\begin{array}{l}96 \\
96\end{array}$ & 8690.00 & 22.60 & 1 \\
\hline 2,4-Pentanediol, 2-methyl- & $107-41-5$ & & 0.58 & miscible & $\begin{array}{l}\text { Lepomis macrochirus } \\
\text { Oncorhynchus mykiss } \\
\text { Orvias latipes }\end{array}$ & $\begin{array}{l}\text { flow-through } \\
\text { flow-through }\end{array}$ & $\begin{array}{l}96 \\
96 \\
96\end{array}$ & $\begin{array}{c}12800.00 \\
9450.00 \\
27.00\end{array}$ & & 1 \\
\hline Diethylamine & $109-89-7$ & 73.14 & 0.58 & miscible & $\begin{array}{l}\text { Pimephales promelas } \\
\text { Poecilia reticulate }\end{array}$ & & $\begin{array}{l}96 \\
96\end{array}$ & & $\begin{array}{l}855.00 \\
130.00\end{array}$ & 1 \\
\hline Dibutyl phosphate & $107-66-4$ & & 0.57 & $1.70 \mathrm{E}+04$ & $\begin{array}{l}\text { Oryzias latipes } \\
\text { Lemis macrochirus }\end{array}$ & semi-static & 96 & & 110.00 & 1 \\
\hline Ethanol, 2-(butoxyethoxy)- & $112-34-5$ & 162.23 & 0.56 & miscible & $\begin{array}{l}\text { Lepomis macrochirus } \\
\text { Leuciscus idus } \\
\text { Menidia beryllina }\end{array}$ & & $\begin{array}{l}48 \\
96\end{array}$ & & $\begin{array}{l}1805.00 \\
2000.00\end{array}$ & 1 \\
\hline Silane, (3-chloropropyl)trimethoxy- & $2530-87-2$ & 199.00 & 0.56 & $6.50 \mathrm{E}+05$ & $\begin{array}{l}\text { Brachydanio rerio } \\
\text { Oncorhvnchus mykiss }\end{array}$ & $\begin{array}{l}\text { semi-static } \\
\text { semi-static }\end{array}$ & $\begin{array}{l}96 \\
96\end{array}$ & $\begin{array}{l}>100 \\
271.00\end{array}$ & & 1 \\
\hline $\begin{array}{l}\text { Silane, trimethoxyphenyl- } \\
\text { 2-Butenal, 3-methyl- }\end{array}$ & $\begin{array}{c}2996-92-1 \\
107-86-8\end{array}$ & & $\begin{array}{l}0.55 \\
0.53\end{array}$ & $\begin{array}{l}6.90 \mathrm{E}+04 \\
1.10 \mathrm{E}+05\end{array}$ & $\begin{array}{l}\text { Oncorhynchus mykiss } \\
\text { Leuciscus idus }\end{array}$ & $\begin{array}{l}\text { flow-through } \\
\text { static }\end{array}$ & 96 & $>0.074$ & 17.60 & $\begin{array}{l}3 \\
1\end{array}$ \\
\hline Triethyleneglycol, monobutylether & $143-22-6$ & & 0.51 & $1.00 \mathrm{E}+06$ & Pimephales promelas & static & 96 & & 2400.00 & 1 \\
\hline Propanol, [(1-methyl-1,2-ethanediyl)bis(oxy)]bis- & $24800-44-0$ & & $0.50-0.60$ & miscible & Oryzias latipes & semi-static & 96 & & $>1000$ & 3 \\
\hline Hydroquinone & $123-31-9$ & & $0.50-0.61$ & $7.30 \mathrm{E}+04$ & $\begin{array}{l}\text { Pimephales promelas } \\
\text { Brachydanio rerio }\end{array}$ & $\begin{array}{l}\text { static } \\
\text { static }\end{array}$ & $\begin{array}{l}96 \\
96\end{array}$ & & $\begin{array}{l}>0.4 \\
0.17\end{array}$ & 1 \\
\hline 1,4-Butanediol & $110-63-4$ & & 0.50 & $1.00 \mathrm{E}+05$ & $\begin{array}{l}\text { Oryzias latipes } \\
\text { Leuciscus idus }\end{array}$ & $\begin{array}{l}\text { semi-static } \\
\text { static }\end{array}$ & $\begin{array}{l}90 \\
96 \\
48\end{array}$ & & $\begin{array}{l}>1100 \\
315.00\end{array}$ & 3 \\
\hline Acrylic acid & $79-10-7$ & & 0.46 & miscible & $\begin{array}{l}\text { Brachydanio rerio } \\
\text { Oncorhynchus mykiss }\end{array}$ & $\begin{array}{l}\text { semi-static } \\
\text { flow-through }\end{array}$ & $\begin{array}{l}96 \\
96\end{array}$ & $\begin{array}{l}222.00 \\
27.00\end{array}$ & & 1 \\
\hline $\begin{array}{l}\text { Tetrahydrofuran } \\
\text { Maleic acid }\end{array}$ & $\begin{array}{l}109-99-9 \\
110-16-7\end{array}$ & $\begin{array}{r}72.11 \\
116.07\end{array}$ & $\begin{array}{l}0.46 \\
0.46\end{array}$ & $4.07 \mathrm{E}+05$ & & & & & & 4 \\
\hline Oxirane, chloromethyl- & $106-89-8$ & & 0.45 & $6.60 \mathrm{E}+04$ & Lepomis macrochirus & static & 96 & & 35.00 & 1 \\
\hline Propionic acid, 3-mercapto- & $107-96-0$ & & 0.43 & $6.03 \mathrm{E}+05$ & $\begin{array}{l}\text { Menidia berylli } \\
\text { Brachydanio rerio }\end{array}$ & $\begin{array}{l}\text { static } \\
\text { semi-static }\end{array}$ & $\begin{array}{l}96 \\
96\end{array}$ & 88.00 & 18.00 & 1 \\
\hline 2-Hydroxyethyl methacrylate & $868-77-9$ & & 0.42 & & $\begin{array}{l}\text { Oryzias latipes } \\
\text { Pimephales promelas }\end{array}$ & $\begin{array}{l}\text { semi-static } \\
\text { flow-through }\end{array}$ & $\begin{array}{l}96 \\
96\end{array}$ & 227.00 & $>100$ & 1 \\
\hline 2-Furaldehyde & $98-01-1$ & & 0.41 & $8.30 \mathrm{E}+04$ & $\begin{array}{l}\text { Pimephales promelas } \\
\text { Lepomis macrochirus }\end{array}$ & & $\begin{array}{l}96 \\
96\end{array}$ & 16.00 & 32.00 & 1 \\
\hline
\end{tabular}


(Table S7. continued)

\begin{tabular}{|c|c|c|c|c|c|c|c|c|c|c|}
\hline Chemicals name & CAS Reg. no & $\begin{array}{c}\text { Molecular } \\
\text { weight } \\
\left(\mathrm{g} \mathrm{mol}^{-1}\right)\end{array}$ & $\log \mathrm{K}_{\mathrm{ow}}$ & Solubility $\left(\mathrm{mg} \mathrm{L}^{-1}\right)$ & Species & Exposure method & $\begin{array}{l}\text { Duration } \\
\text { (h) }\end{array}$ & $\begin{array}{c}\text { Measured } \\
\mathrm{LC}_{50} \\
\left(\mathrm{mg} \mathrm{L}^{-1}\right) \\
\end{array}$ & $\begin{array}{c}\text { Nominal } \\
\mathrm{LC}_{50} \\
\left(\mathrm{mg} \mathrm{L}^{-1}\right) \\
\end{array}$ & Classification \\
\hline N-Vinyl-pyrrolidinone & $88-12-0$ & & 0.40 & & $\begin{array}{l}\text { Oncorhyncus mykiss } \\
\text { Oncorhyncus mykiss }\end{array}$ & & $\begin{array}{l}96 \\
72\end{array}$ & $\begin{array}{l}913.00 \\
976.00\end{array}$ & & 1 \\
\hline Ether, methyl vinyl & $107-25-5$ & 58.08 & 0.40 & $1.71 \mathrm{E}+04$ & & & & & & 4 \\
\hline tert-Butylamine & $75-64-9$ & & 0.40 & & $\begin{array}{l}\text { Oncorhynchus mykiss } \\
\text { Oncorhynchus mykiss }\end{array}$ & & $\begin{array}{l}96 \\
96\end{array}$ & & $\begin{array}{r}28.00 \\
270.00\end{array}$ & 1 \\
\hline 2-Propanol, 1-methoxy-, acetate & $108-65-6$ & & 0.36 & $1.00 \mathrm{E}+05$ & $\begin{array}{l}\text { Oryzias latipes } \\
\text { Pimephales promelas }\end{array}$ & $\begin{array}{l}\text { semi-static } \\
\text { static }\end{array}$ & $\begin{array}{l}96 \\
96\end{array}$ & & $\begin{array}{l}>100 \\
161.00\end{array}$ & 1 \\
\hline Ethanol, 2-tert-butoxy- & $7580-85-0$ & 118.17 & 0.36 & $1.00 \mathrm{E}+05$ & $\begin{array}{l}\text { Prizias latipes } \\
\text { Orelas }\end{array}$ & $\begin{array}{l}\text { semi-static } \\
\text { stanc }\end{array}$ & 96 & & $>100$ & 3 \\
\hline Acrylic acid, monoester with 1,2-propanediol & $25584-83-2$ & 130.14 & 0.35 & $3.07 \mathrm{E}+05$ & fathead minnows & & 96 & $\begin{array}{r}3.10 \\
2480\end{array}$ & & 1 \\
\hline Formaldehyde & $50-00-0$ & & 0.35 & & $\begin{array}{l}\text { Ictalurus melas } \\
\text { Lepomis cyanellus } \\
\text { Lepomis macrochirus }\end{array}$ & $\begin{array}{l}\text { flow through } \\
\text { flow through }\end{array}$ & $\begin{array}{l}96 \\
96\end{array}$ & $\begin{array}{l}24.80 \\
69.20 \\
40.00\end{array}$ & & 1 \\
\hline & & & & & Carassius auratus & flow through & 96 & 121.00 & & \\
\hline 2,4-Pentanedione & $123-54-6$ & 100.12 & $0.34-0.4$ & $1.66 \mathrm{E}+05$ & $\begin{array}{l}\text { Ictalurus punctatus } \\
\text { Lepomis macrochirus }\end{array}$ & $\begin{array}{l}\text { flow through } \\
\text { flow through }\end{array}$ & $\begin{array}{l}96 \\
96\end{array}$ & $\begin{array}{l}106.00 \\
60.10\end{array}$ & & 1 \\
\hline 2,4-Pentanedione & & & $0.34-0.4$ & & Pimephales promelas & flow through & 96 & 104.00 & & \\
\hline Propane, 1-(allyloxy)-2,3-epoxy- & $106-92-3$ & & 0.34 & $1.28 \mathrm{E}+05$ & Cyprinus carpio & & 96 & 36.00 & & 1 \\
\hline Propanal, 3-(methylthio)- & $3268-49-3$ & & 0.34 & $7.79 \mathrm{E}+04$ & Brachydanio rerio & static & 24 & & 14.00 & 1 \\
\hline Propanoic acid, anhydride & $123-62-6$ & & 0.33 & & & static & 96 & & 51.80 & 1 \\
\hline & $120-0 z-0$ & & & & & $\begin{array}{l}\text { Static } \\
\text { flow-through }\end{array}$ & 96 & 67.10 & 85.30 & 1 \\
\hline & & & & & Pimephales promelas & static & 96 & 51.80 & & \\
\hline Propionic acid & 79-09-4 & & 0.33 & miscible & $\begin{array}{l}\text { Lepomis macrochirus } \\
\text { Oncorhynchus mykiss }\end{array}$ & $\begin{array}{l}\text { static } \\
\text { flow-through }\end{array}$ & $\begin{array}{l}96 \\
96\end{array}$ & $\begin{array}{l}85.30 \\
67.10\end{array}$ & & 1 \\
\hline 2-(2-Ethoxyethoxy)ethyl acetate & $112-15-2$ & 176.21 & 0.32 & $1.00 \mathrm{E}+06$ & Oncornynchus mykiss & flow-through & 90 & & & 4 \\
\hline 3-Butyn-2-ol, 2-methyl- & $115-19-5$ & & 0.32 & miscible & $\begin{array}{l}\text { Pimephales promelas } \\
\text { Leuciscus idus }\end{array}$ & & $\begin{array}{l}96 \\
96\end{array}$ & & $\begin{array}{l}3290.00 \\
2200-4600\end{array}$ & 1 \\
\hline $\begin{array}{l}\text { Propionitrile, 3-(triethoxysilyl)- } \\
\text { Pentaerythritol }\end{array}$ & $\begin{array}{l}919-31-3 \\
115-77-5\end{array}$ & & $\begin{array}{l}0.32 \\
0.30\end{array}$ & $\begin{array}{l}1.00 \mathrm{E}+06 \\
2.50 \mathrm{E}+04\end{array}$ & $\begin{array}{l}\text { Leuciscus idus } \\
\text { Oncorhynchus mykiss } \\
\text { Oryzias latipes }\end{array}$ & $\begin{array}{l}\text { flow-through } \\
\text { semi-static }\end{array}$ & $\begin{array}{l}96 \\
96 \\
96\end{array}$ & $\begin{array}{l}>110 \\
>100\end{array}$ & & $\begin{array}{l}3 \\
3 \\
2\end{array}$ \\
\hline Isocyanuric acid & $108-80-5$ & & 0.30 & $2.70 \mathrm{E}+03$ & Oryzias latipes & semi-static & 96 & $>100$ & & 3 \\
\hline 2-Butanone & $78-93-3$ & 72.11 & 0.29 & $2.76 \mathrm{E}+05$ & $\begin{array}{l}\text { Pimephales promelas } \\
\text { Lepomis macrochirus }\end{array}$ & $\begin{array}{l}\text { flow-through } \\
\text { static }\end{array}$ & $\begin{array}{l}96 \\
48\end{array}$ & 3200.00 & 5640.00 & 1 \\
\hline Acetic acid, mercapto- & $68-11-1$ & & 0.27 & $>1.00 \mathrm{E}+04$ & Oncorhynchus mykiss & flow through & 96 & $>100$ & & 3 \\
\hline 2-Propanamine & $75-31-0$ & & 0.26 & & Oncorhynchus mykiss & & 96 & & 40.00 & 1 \\
\hline Ethyl acetoacetate & $141-97-9$ & & 0.25 & $1.25 \mathrm{E}+05$ & Lenciscus idus. & & 48 & & 275.00 & 1 \\
\hline Silanetriol, methyl-, triacetate & $4253-34-3$ & 220.00 & 0.25 & $9.10 \mathrm{E}+04$ & $\begin{array}{l}\text { Brachydanio rerio } \\
\text { Carassius auratus }\end{array}$ & $\begin{array}{l}\text { semi-static } \\
\text { flow through }\end{array}$ & $\begin{array}{l}96 \\
96\end{array}$ & $\begin{array}{l}251.00 \\
100.00\end{array}$ & & 1 \\
\hline 1-Propanol & $71-23-8$ & & 0.25 & & & & & & & 4 \\
\hline Trimethylamine & $75-50-3$ & & 0.25 & $4.10 \mathrm{E}+05$ & $\begin{array}{l}\text { Leuciscus idus } \\
\text { Oryzias latipes }\end{array}$ & static & $\begin{array}{l}48 \\
48\end{array}$ & & $\begin{array}{c}610.00 \\
1000.00\end{array}$ & 1 \\
\hline 4-Piperidinol, 2,2,6,6-tetramethyl- & $2403-88-5$ & & 0.24 & $1.00 \mathrm{E}+05$ & $\begin{array}{l}\text { Brachydanio rerio } \\
\text { Oryzias latipes }\end{array}$ & & $\begin{array}{l}96 \\
96\end{array}$ & & $\begin{array}{l}>1000 \\
237.00\end{array}$ & 1 \\
\hline Dimethyl carbonate & $616-38-6$ & 90.08 & 0.23 & & & & & & & 4 \\
\hline Chloroacetic acid & $79-11-8$ & 94.50 & 0.22 & $4.26 \mathrm{E}+03$ & $\begin{array}{l}\text { Pimephales promelas } \\
\text { Poecilia reticulata }\end{array}$ & $\begin{array}{l}\text { semi-static } \\
\text { static }\end{array}$ & $\begin{array}{l}96 \\
96\end{array}$ & 145.00 & 370.00 & 1 \\
\hline Ethanol, 2-(diethylamino)- & $100-37-8$ & & 0.21 & miscible & Pimephales promelas & flow-through & 96 & 1780.00 & & 1 \\
\hline Difluoromethane & $75-10-5$ & & 0.21 & $4.40 \mathrm{E}+03$ & Leuciscus idus & & & 147.00 & & 4 \\
\hline Acetin, tri- & $102-76-1$ & & 0.21 & $7.00 \mathrm{E}+04$ & $\begin{array}{l}\text { Oryzias latipes } \\
\text { Pimephales promelas }\end{array}$ & $\begin{array}{l}\text { semi-static } \\
\text { static } \\
\text { static }\end{array}$ & $\begin{array}{l}96 \\
96 \\
48\end{array}$ & 165.30 & $>100.0$ & 1 \\
\hline Nitric acid & 7697-37-2 & 62.00 & 0.21 & very soluble & $\begin{array}{l}\text { Cyprinus carpio } \\
\text { Gambusia affinis }\end{array}$ & $\begin{array}{l}\text { static } \\
\text { static }\end{array}$ & $\begin{array}{l}48 \\
96\end{array}$ & & $\begin{array}{l}174.00 \\
72.00\end{array}$ & 1 \\
\hline Methyl acetate & $79-20-9$ & 74.08 & 0.18 & $2.50 \mathrm{E}+05-2.95 \mathrm{E}+05$ & $\begin{array}{l}\text { Leuciscus idus } \\
\text { Pimephales promelas }\end{array}$ & & $\begin{array}{l}48 \\
96\end{array}$ & $\begin{array}{l}225.00 \\
320.00\end{array}$ & & 1 \\
\hline m-Phenylene-bis(methylamine) & $1477-55-0$ & & 0.18 & $1.00 \mathrm{E}+05$ & $\begin{array}{l}\text { Pimephales promelas } \\
\text { medaka } \\
\text { golden orfe }\end{array}$ & & $\begin{array}{l}96 \\
96 \\
96\end{array}$ & $\begin{array}{l}>100 \\
87.60 \\
75.00\end{array}$ & & 1 \\
\hline Stannane, butyltrichloro- & $1118-46-3$ & & 0.18 & $1.00 \mathrm{E}+03-1.00 \mathrm{E}+04$ & Brachydanio rerio & semi-static & 96 & $>100$ & & 3 \\
\hline Butanol, 3-methoxy-3-methyl- & $56539-66-3$ & & 0.18 & $1.00 \mathrm{E}+05$ & Orizias latipes & semi-static & 96 & & $>100$ & 3 \\
\hline Ethane, nitro- & $79-24-3$ & & 0.18 & $4.50 \mathrm{E}+04$ & Pimephales promelas & static & 96 & 596.00 & & 1 \\
\hline
\end{tabular}


(Table S7. continued)

\begin{tabular}{|c|c|c|c|c|c|c|c|c|c|c|}
\hline Chemicals name & CAS Reg. no & $\begin{array}{c}\text { Molecular } \\
\text { weight } \\
\left(\mathrm{g} \mathrm{mol}^{-1}\right)\end{array}$ & $\log \mathrm{K}_{\mathrm{ow}}$ & Solubility $\left(\mathrm{mg} \mathrm{L}^{-1}\right)$ & Species & Exposure method & $\begin{array}{l}\text { Duration } \\
\text { (h) }\end{array}$ & $\begin{array}{c}\text { Measured } \\
\mathrm{LC}_{50} \\
\left(\mathrm{mg} \mathrm{L}^{-1}\right) \\
\end{array}$ & $\begin{array}{c}\text { Nominal } \\
\mathrm{LC}_{50} \\
\left(\mathrm{mg} \mathrm{L}^{-1}\right) \\
\end{array}$ & Classification \\
\hline 2-Propen-1-ol & $107-18-6$ & 58.08 & 0.17 & miscible & $\begin{array}{l}\text { Oryzias latipes } \\
\text { Pimephales promelas }\end{array}$ & $\begin{array}{l}\text { semi-static } \\
\text { flow-through }\end{array}$ & $\begin{array}{l}96 \\
96\end{array}$ & $\begin{array}{l}0.59 \\
0.32\end{array}$ & & 1 \\
\hline Dimethyl sulfate & $77-78-1$ & & 0.16 & $2.80 \mathrm{E}+04$ & $\begin{array}{l}\text { Lepomis macrochirus } \\
\text { Leuciscus idus } \\
\text { melanotus }\end{array}$ & $\begin{array}{l}\text { static } \\
\text { static }\end{array}$ & $\begin{array}{l}96 \\
96\end{array}$ & & $\begin{array}{l}7.50 \\
14.00\end{array}$ & 1 \\
\hline $\begin{array}{l}\text { Formic acid, chloro-, methyl ester } \\
\text { 1,3-Propanediol, 2,2-dimethyl- } \\
\text { 2H-Azepin-2-one, hexahydro- }\end{array}$ & $\begin{array}{l}79-22-1 \\
126-30-7 \\
105-60-2\end{array}$ & 104.15 & $\begin{array}{l}0.14 \\
0.12 \\
0.12\end{array}$ & $\begin{array}{l}9.28 \mathrm{E}+05 \\
1.90 \mathrm{E}+03 \\
456 \mathrm{E}+06\end{array}$ & $\begin{array}{l}\text { Leuciscus idus } \\
\text { Oryzias latipes } \\
\text { Salmo gaipdneri }\end{array}$ & $\begin{array}{l}\text { static } \\
\text { Semi-static } \\
\text { static }\end{array}$ & $\begin{array}{l}96 \\
96\end{array}$ & & $\begin{array}{c}4.50 \\
>>1000 \\
>\end{array}$ & $\begin{array}{l}1 \\
3 \\
1\end{array}$ \\
\hline 2H-Azepin-2-one, hexahydro- & $105-60-2$ & & & $4.56 \mathrm{E}+06$ & $\begin{array}{l}\text { Salmo gairdneri } \\
\text { Pimonales }\end{array}$ & static & 96 & & $>500<1000$ & \\
\hline Adipic acid & $124-04-9$ & & 0.09 & $2.30 \mathrm{E}+04$ & $\begin{array}{l}\text { Pimephales promelas } \\
\text { Danio rerio }\end{array}$ & $\begin{array}{l}\text { static } \\
\text { static }\end{array}$ & $\begin{array}{l}96 \\
96\end{array}$ & & $\begin{array}{l}97.00 \\
>1000\end{array}$ & 1 \\
\hline Cyclohexanone & $108-94-1$ & 98.14 & 0.08 & $2.30 \mathrm{E}+04$ & $\begin{array}{l}\text { Idus idus melanotous } \\
\text { Pimephales promelas }\end{array}$ & $\begin{array}{l}\text { Static } \\
\text { flow-through }\end{array}$ & $\begin{array}{l}96 \\
96\end{array}$ & & $\begin{array}{l}536-752 \\
527.00 \\
73200\end{array}$ & 1 \\
\hline Benzenesulfonic acid, 4,4'-oxybis-, dihydrazide & $80-51-3$ & & 0.08 & $6.25 \mathrm{E}+01$ & $\begin{array}{l}\text { Oryzias latipes } \\
\text { Oryzias latipes }\end{array}$ & $\begin{array}{l}\text { static } \\
\text { semi-static }\end{array}$ & $\begin{array}{l}96 \\
96\end{array}$ & $>6.6$ & 74.00 & 2 \\
\hline Morpholine, 4-ethyl- & $100-74-3$ & & 0.08 & $3.03 \mathrm{E}+05$ & $\begin{array}{l}\text { Orizias latipes } \\
\text { Leuciscus idus }\end{array}$ & $\begin{array}{l}\text { semi-static } \\
\text { static }\end{array}$ & $\begin{array}{l}96 \\
96\end{array}$ & $>100$ & 280.00 & 1 \\
\hline Ethanol, 2-propoxy- & $2807-30-9$ & & 0.08 & completely soluble & $\begin{array}{l}\text { Pimephales promelas } \\
\text { Pimephales promelas }\end{array}$ & static & $\begin{array}{l}96 \\
96\end{array}$ & 1420.00 & 2137.00 & 1 \\
\hline Toluene, 2,4-diamine & $95-80-7$ & & 0.07 & $3.80 \mathrm{E}+04$ & $\begin{array}{l}\text { Oryzias latipes } \\
\text { Pagrus major }\end{array}$ & & $\begin{array}{l}96 \\
96\end{array}$ & $\begin{array}{l}912.00 \\
0.2-0.4\end{array}$ & & 1 \\
\hline Phosphoryl trichloride & $10025-87-3$ & 153.33 & 0.06 & & & & & & & 4 \\
\hline Oxirane, methyl- & 75-56-9 & 58.08 & 0.06 & $3.95 \mathrm{E}+05-4.05 \mathrm{E}+05$ & $\begin{array}{l}\text { Lepomis macrochirus } \\
\text { Oncorhvnchus mykiss }\end{array}$ & $\begin{array}{l}\text { static } \\
\text { semi-static }\end{array}$ & $\begin{array}{l}96 \\
96\end{array}$ & & $\begin{array}{c}215.00 \\
52.00\end{array}$ & 1 \\
\hline $\begin{array}{l}\text { 2-Propanol } \\
\text { Phenol, 4-amino- }\end{array}$ & $\begin{array}{l}67-63-0 \\
123-30-8\end{array}$ & & $\begin{array}{l}0.05 \\
0.04\end{array}$ & $\begin{array}{l}1.00 \mathrm{E}+06 \\
1.57 \mathrm{E}+04\end{array}$ & $\begin{array}{l}\text { Pimephales promelas } \\
\text { Oryzias latipes }\end{array}$ & $\begin{array}{l}\text { flow-through } \\
\text { flow-through }\end{array}$ & $\begin{array}{l}90 \\
96\end{array}$ & $\begin{array}{c}9640.00 \\
0.93\end{array}$ & & $\begin{array}{l}1 \\
1\end{array}$ \\
\hline Silicic acid, (H4SiO4), tetraethyl ester & $78-10-4$ & & 0.04 & & & & & & & 4 \\
\hline Ethanol, 2-(1-methylethoxy)- & $109-59-1$ & & 0.04 & $1.00 \mathrm{E}+05$ & Oryzias latipes & semi-static & 96 & & $>100$ & 3 \\
\hline $\begin{array}{l}\text { 1,2-Benzenedicarboxylic acid, bis(2-methoxyethyl) ester } \\
\text { (Di(methoxyethyl)phthalate) }\end{array}$ & $117-82-8$ & & 0.04 & $8.50 \mathrm{E}+00$ & & & & & & 4 \\
\hline Methyl formate & $107-31-3$ & & 0.03 & $2.40 \mathrm{E}+04$ & $\begin{array}{l}\text { Leuciscus idus } \\
\text { Poecilia reticulata }\end{array}$ & static & $\begin{array}{l}96 \\
48\end{array}$ & & $\begin{array}{c}115.00 \\
100-500\end{array}$ & 1 \\
\hline 1,6-Hexanediamine & $124-09-4$ & 116.24 & 0.02 & $8.00 \mathrm{E}+02$ & $\begin{array}{l}\text { Lepomis macrochirus } \\
\text { Pimephales promelas }\end{array}$ & $\begin{array}{l}\text { static } \\
\text { static }\end{array}$ & $\begin{array}{l}48 \\
96\end{array}$ & & $\begin{array}{c}73.50 \\
1825.00\end{array}$ & 1 \\
\hline Toluene-2,3-diamine & $2687-25-4$ & 122.17 & 0.01 & $1.39 \mathrm{E}+04$ & & & & & & 4 \\
\hline Propanol, 1(or 2)-(2-methoxymethylethoxy)- & $34590-94-8$ & & 0.01 & & $\begin{array}{l}\text { Pimephales promelas } \\
\text { Notropis atherinoides }\end{array}$ & & $\begin{array}{l}96 \\
72\end{array}$ & & $\begin{array}{l}>10000 \\
>150\end{array}$ & 3 \\
\hline Chlorosulfuric acid & 7790-94-5 & 116.52 & 0.00 & & & & & & & 4 \\
\hline Theophylline & $58-55-9$ & & -0.01 & $5.50 \mathrm{E}+03-8.30 \mathrm{E}+03$ & Leuciscus idus & static & 96 & & 100.00 & 1 \\
\hline Acrolein & $107-02-8$ & 56.06 & -0.01 & $2.06 \mathrm{E}+05-2.70 \mathrm{E}+05$ & $\begin{array}{l}\text { Pimephales promelas } \\
\text { Oncorhynchus mykiss }\end{array}$ & & $\begin{array}{l}96 \\
96\end{array}$ & $\begin{array}{l}0.00 \\
0.00\end{array}$ & & 1 \\
\hline Glutaraldehyde & $111-30-8$ & & -0.01 & miscible & $\begin{array}{l}\text { Bluegill sunfish } \\
\text { Leucisus idus }\end{array}$ & $\begin{array}{l}\text { static } \\
\text { static }\end{array}$ & 96 & & $\begin{array}{r}11.20 \\
350.00\end{array}$ & 1 \\
\hline Phosphonic acid, (1-hydroxyethylidene)bis- & 2809-21-4 & 206.03 & -0.01 & $6.90 \mathrm{E}+05$ & $\begin{array}{l}\text { Leuciscus idus } \\
\text { Oncorhynchus mykiss }\end{array}$ & $\begin{array}{l}\text { static } \\
\text { flow-through }\end{array}$ & $\begin{array}{l}90 \\
96\end{array}$ & 180.00 & 207.00 & 1 \\
\hline $\begin{array}{l}\text { 1H-Imidazole } \\
\text { 1,5-Pentanediol, 3-methyl- }\end{array}$ & $\begin{array}{r}288-32-4 \\
4457-71-0\end{array}$ & & $\begin{array}{l}-0.02 \\
-0.03\end{array}$ & $\begin{array}{l}6.63 \mathrm{E}+05 \\
\text { miscible } \\
\text { micishe }\end{array}$ & $\begin{array}{l}\text { Leuciscus idus } \\
\text { Oryzias latipes }\end{array}$ & $\begin{array}{l}\text { static } \\
\text { semi-static }\end{array}$ & $\begin{array}{l}96 \\
96\end{array}$ & $>100$ & 283.60 & $\begin{array}{l}1 \\
3\end{array}$ \\
\hline Propanenitrile, 2-hydroxy-2-methyl- & $75-86-5$ & & -0.03 & miscible & Oncorhynchus mykiss & & 96 & & 0.22 & 1 \\
\hline Silane, ethenyltrimethoxy- & $2768-02-7$ & 148.23 & -0.03 & $5.04 \mathrm{E}+05$ & $\begin{array}{l}\text { Brachydanio rerio } \\
\text { Brachydanio rerio }\end{array}$ & $\begin{array}{l}\text { semi-static } \\
\text { static }\end{array}$ & $\begin{array}{l}96 \\
96\end{array}$ & & $\begin{array}{l}>100 \\
>100\end{array}$ & 3 \\
\hline $\begin{array}{l}\text { Dimethyl malonate } \\
\text { Ethanol, 2-mercapto- }\end{array}$ & $\begin{array}{l}108-59-8 \\
60-24-2\end{array}$ & $\begin{array}{l}132.12 \\
78.13\end{array}$ & $\begin{array}{l}-0.05 \\
-0.06\end{array}$ & $\begin{array}{c}9.90 \mathrm{E}+04 \\
\text { miscible }\end{array}$ & $\begin{array}{l}\text { Brachydanio rerio } \\
\text { Leuciscus idus }\end{array}$ & $\begin{array}{l}\text { flow through } \\
\text { static }\end{array}$ & $\begin{array}{l}96 \\
96\end{array}$ & $\begin{array}{l}21.00 \\
37.00\end{array}$ & & $\begin{array}{l}1 \\
1\end{array}$ \\
\hline Caffeine & $58-08-2$ & 194.19 & -0.09 & $2.00 \mathrm{E}+01$ & $\begin{array}{l}\text { Leuciscus idus } \\
\text { Pimephales promelas }\end{array}$ & $\begin{array}{l}\text { static } \\
\text { static }\end{array}$ & $\begin{array}{c}96 \\
120\end{array}$ & & $\begin{array}{c}87.00 \\
720.00\end{array}$ & 2 \\
\hline $\begin{array}{l}\text { 2-Furanmethanol, tetrahydro- } \\
\text { Ethanamine }\end{array}$ & $\begin{array}{l}97-99-4 \\
75-04-7\end{array}$ & 102.13 & $\begin{array}{l}-0.11 \\
-0.13\end{array}$ & $2.50 \mathrm{E}+05$ & $\begin{array}{l}\text { Oryzias latipes } \\
\text { Pimephales promelas }\end{array}$ & semi-static & $\begin{array}{l}96 \\
96\end{array}$ & $\begin{array}{l}>101 \\
227.00\end{array}$ & & $\begin{array}{l}3 \\
1\end{array}$ \\
\hline
\end{tabular}


(Table S7. continued)

\begin{tabular}{|c|c|c|c|c|c|c|c|c|c|c|}
\hline Chemicals name & CAS Reg. no & $\begin{array}{c}\text { Molecular } \\
\text { weight } \\
\left(\mathrm{g} \mathrm{mol}^{-1}\right)\end{array}$ & $\log K_{\text {ow }}$ & Solubility $\left(\mathrm{mg} \mathrm{L}^{-1}\right)$ & Species & Exposure method & $\begin{array}{l}\text { Duration } \\
\text { (h) }\end{array}$ & $\begin{array}{c}\text { Measured } \\
\mathrm{LC}_{50} \\
\left(\mathrm{mg} \mathrm{L}^{-1}\right)\end{array}$ & $\begin{array}{c}\text { Nominal } \\
\text { LC } \\
\left(\mathrm{mg} \mathrm{L}^{-1}\right) \\
\end{array}$ & Classification \\
\hline \multirow{3}{*}{ Acetaldehyde, oxime } & \multirow{3}{*}{ 107-29-9 } & \multirow{3}{*}{59.07} & \multirow{3}{*}{-0.13} & \multirow{3}{*}{$>1.00 \mathrm{E}+04$} & Lepomis macrochirus & static & 96 & & 0.02 & \multirow{3}{*}{1} \\
\hline & & & & & $\begin{array}{l}\text { Pimephales promelas } \\
\text { Salmo oairdneri }\end{array}$ & $\begin{array}{l}\text { static } \\
\text { static }\end{array}$ & $\begin{array}{l}96 \\
96\end{array}$ & & $\begin{array}{l}76.00 \\
0.03\end{array}$ & \\
\hline & & & & & $\begin{array}{l}\text { Salmo gallanerl } \\
\text { Oryzias latipes }\end{array}$ & $\begin{array}{l}\text { static } \\
\text { semi-static }\end{array}$ & $\begin{array}{l}96 \\
96\end{array}$ & & $>100$ & \\
\hline \multirow{2}{*}{ Diacetone alcohol } & \multirow{2}{*}{$123-42-2$} & \multirow{2}{*}{116.16} & \multirow{2}{*}{-0.14} & \multirow{2}{*}{$1.00 \mathrm{E}+05$} & Lepomis macrochirus & static & 96 & & 420.00 & \multirow{2}{*}{1} \\
\hline & & & & & Menidia beryllina & static & 96 & & 420.00 & \\
\hline 2-Propanol, 1,1',1"-nitrilotri- & $122-20-3$ & 191.27 & -0.15 & miscible & $\begin{array}{l}\text { Cyprinus carpio } \\
\text { Pimephales promelas }\end{array}$ & $\begin{array}{l}\text { semi-static } \\
\text { static }\end{array}$ & $\begin{array}{l}96 \\
96\end{array}$ & $>1000$ & $>100$ & 3 \\
\hline 2-Propenamide, 2-methyl- & $79-39-0$ & 85.10 & -0.15 & $1.00 \mathrm{E}+05$ & Oryzias latipes & semistatic & 96 & $>100$ & 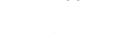 & 1 \\
\hline & 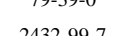 & 0.10 & $\begin{array}{l}-0.15 \\
0.16\end{array}$ & $1.00 \mathrm{D}+\mathrm{DS}$ & Leuciscus idus & static & 48 & & 2730.00 & 1 \\
\hline $\begin{array}{l}\text { Undecanoic acid, 11-amino- } \\
\text { 2-Propanol 1-[2-(2-methoxy-1-methylethoxy)-1-methylethoxy]- }\end{array}$ & 2432-99-7 & 201.31 & -0.16 & $8.00 \mathrm{E}+02$ & Brachydanio rerio & & 96 & $>833$ & & 3 \\
\hline $\begin{array}{l}\text { 2-Propanol, 1-[2-(2-methoxy-1-methylethoxy)-1-methylethoxy]- } \\
\text { Ethanol, 2-(2-propoxyethoxy)- }\end{array}$ & $\begin{array}{c}20324-33-8 \\
6881-94-3\end{array}$ & $\begin{array}{l}206.28 \\
148.20\end{array}$ & $\begin{array}{l}-0.20 \\
-0.20\end{array}$ & $1.00 \mathrm{E}+06$ & & & & & & $\begin{array}{l}4 \\
4\end{array}$ \\
\hline 2-Hydroxyethyl acrylate & $818-61-1$ & 116.12 & -0.21 & . & Pimephales promelas & flow through & 96 & 4.80 & & 1 \\
\hline 2,3-Dibromosuccinic acid & $526-78-3$ & 100.12 & 0.21 & & Cyprinodon variegatus & flow through & 96 & 17.50 & & 1 \\
\hline 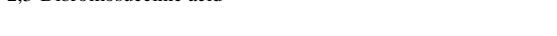 & (2) & & & $2.00 \mathrm{E}+04$ & Salvelinus fontinalis & flow-through & 96 & 6070.00 & & 4 \\
\hline Acetone & $67-64-1$ & 58.08 & -0.24 & $1.00 \mathrm{E}+06$ & Lepomis macroc & flow-through & 96 & 7300.00 & & 1 \\
\hline & & & & & $\begin{array}{l}\text { Pimephales promelas } \\
\text { Oncorhynchus mykiss }\end{array}$ & $\begin{array}{l}\text { flow-through } \\
\text { static }\end{array}$ & $\begin{array}{l}96 \\
96\end{array}$ & 9100.00 & 5540.00 & 1 \\
\hline & & & & & Pimephales promelas & flow-through & 96 & 9850.00 & 000.00 & \\
\hline 1,4-Dioxane & $123-91-1$ & 88.11 & -0.27 & miscible & Oryzias latipes & semi-static & 96 & & 10500.00 & 1 \\
\hline & & & & & Pimephales promelas & static & 96 & & 13000.00 & \\
\hline & & & & & Salmo gairdneri & static & 96 & 13000.00 & & \\
\hline Ethanol & $64-17-5$ & 46.07 & -0.31 & miscible & $\begin{array}{l}\text { Pimephales promelas } \\
\text { Pimephales promelas }\end{array}$ & $\begin{array}{l}\text { flow-through } \\
\text { static }\end{array}$ & $\begin{array}{l}96 \\
96\end{array}$ & $\begin{array}{l}13480.00 \\
>100\end{array}$ & & 1 \\
\hline & & & & & Lepomis macrochirus & static & 96 & & 720.00 & \\
\hline 1,4-Dicyanobutane & $111-69-3$ & 108.14 & -0.32 & $8.30 \mathrm{E}+04$ & Onchorynchus mykiss & static & 96 & & 670.00 & 1 \\
\hline & & & & & Leuciscus idus & $\begin{array}{l}\text { static } \\
\text { emic }\end{array}$ & 48 & & 384.00 & \\
\hline Propanenitrile, 2-hydroxy- & $\begin{array}{r}78-97-7 \\
75-52-5\end{array}$ & & -0.32 & $\begin{array}{l}\text { infinite } \\
111005\end{array}$ & Oryzias latipes & semi-static & 96 & & 0.90 & 1 \\
\hline $\begin{array}{l}\text { Methane, nitro- } \\
\text { Pathed }\end{array}$ & $75-52-5$ & & -0.33 & $1.11 \mathrm{E}+05$ & & & & & & 4 \\
\hline Butanedioic acid, methylene- & $97-65-4$ & & -0.34 & $8.30 \mathrm{E}+04$ & Salmo gairdneri & static & 24 & & 190.00 & 1 \\
\hline 1,2-Butylene glycol & $584-03-2$ & 90.14 & -0.34 & $1.00 \mathrm{E}+05$ & Oryzias latipes & & 96 & & $>1000$ & 3 \\
\hline & & & & & Pimephales promelas & static & 96 & & $1,000-1,150$ & \\
\hline Acetonitrile & $75-05-8$ & 41.05 & -0.34 & $1.00 \mathrm{E}+06$ & Pimephales promelas & static & 96 & & $>100$ & 1 \\
\hline & & & & & Pimephales promeles & flow-through & 96 & 1640.00 & & \\
\hline Oxydiethylene chloroformate & $106-75-2$ & & -0.34 & $3.00 \mathrm{E}+00$ & Leuciscus idus & static & 96 & 00000 & 1.78 & 1 \\
\hline Propane, 1-Amino-3-dimethylamino- & $109-55-7$ & & -0.35 & miscible & Leuciscus idus & static & 96 & & 122.00 & 1 \\
\hline Nicotinamide & 98-92-0 & 122.13 & -0.38 & $6.91 \mathrm{E}+05-1.00 \mathrm{E}+06$ & $\begin{array}{l}\text { Poecilia reticulata, } \\
\text { Teleostei Poeciliidae }\end{array}$ & static & 96 & $>1000$ & & 3 \\
\hline Dimethylamine & $124-40-3$ & 45.08 & -0.38 & & & & & & & 4 \\
\hline Propylamine, 3-methoxy- & 5332-73-0 & 89.14 & -0.42 & & Leuciscus idus & & 96 & 148.00 & & 1 \\
\hline 2-Propanol, 1-methoxy- & $107-98-2$ & 90.12 & -0.44 & $2.00 \mathrm{E}+05$ & $\begin{array}{l}\text { Leuciscus idus } \\
\text { Pimphages promelas }\end{array}$ & $\begin{array}{l}\text { static } \\
\text { static }\end{array}$ & 96 & & $4600-10000$ & 1 \\
\hline & & & & & $\begin{array}{l}\text { Pimephales promelas } \\
\text { Oncorhynchus mykiss }\end{array}$ & $\begin{array}{l}\text { Slatic } \\
\text { static }\end{array}$ & $\begin{array}{l}96 \\
96\end{array}$ & $>500$ & 20800.00 & \\
\hline & & & & & Lepomis macrochirus & static & 96 & & 832.00 & \\
\hline & & & & & Leuciscus idus & static & 96 & & 4030.00 & \\
\hline 2-Pyrrolidinone, 1-methyl- & $872-50-4$ & 99.13 & -0.46 & $1.00 \mathrm{E}+06$ & Oncorhynchus mykiss & static & 96 & & 3048.00 & 1 \\
\hline & & & & & Pimephales promelas & static & 96 & & 1072.00 & \\
\hline & & & & & Poecilia reticulata & static & 96 & & 2670.00 & \\
\hline & & & & & Lenciscus idus & & 96 & & 4000.00 & \\
\hline Trimethyl phosphate & $512-56-1$ & & -0.46 & miscible & Oryzias latipes & Semi-static & 96 & & $>1,050$ & 3 \\
\hline 1,3-Propanediol, 2-ethyl-2-(hydroxymethyl)- & $77-99-6$ & 134.20 & -0.47 & $>1.00 \mathrm{E}+05$ & $\begin{array}{l}\text { Leuciscus idus } \\
\text { Oryzias latipes }\end{array}$ & semi-static & $\begin{array}{l}48 \\
96\end{array}$ & & $\begin{array}{l}>=1000 \\
>>1000\end{array}$ & 1 \\
\hline Guanidine, cyano- & $461-58-5$ & 84.08 & -0.52 & $4.00 \mathrm{E}+04$ & $\begin{array}{l}\text { Oryzias latipes } \\
\text { Oycorhynchus mykiss }\end{array}$ & $\begin{array}{l}\text { semistatic } \\
\text { static }\end{array}$ & $\begin{array}{l}96 \\
96\end{array}$ & $>100$ & & 3 \\
\hline & & & & & $\begin{array}{l}\text { Oncorhynchus mykiss } \\
\text { Lepomis macrochirus }\end{array}$ & $\begin{array}{l}\text { static } \\
\text { semi-static }\end{array}$ & 96 & $\begin{array}{l}1.60 \\
1.10\end{array}$ & & \\
\hline Peroxyacetic acid & $79-21-0$ & 76.05 & -0.52 & $1.00 \mathrm{E}+06$ & Oncorhynchus mykiss & static & 96 & & 1.00 & 1 \\
\hline & & & & & Oncorhynchus mykiss & semi-static & 96 & & 2.00 & \\
\hline Ethaneamine, 2'-oxibis[N,N-dimethyl-] & $3033-62-3$ & 160.26 & -0.54 & $1.00 \mathrm{E}+06$ & Brachydanio rerio & semi-static & 96 & 131.00 & & 1 \\
\hline
\end{tabular}


(Table S7. continued)

\begin{tabular}{|c|c|c|c|c|c|c|c|c|c|c|}
\hline Chemicals name & CAS Reg. no & $\begin{array}{c}\text { Molecular } \\
\text { weight } \\
\left(\mathrm{g} \mathrm{mol}{ }^{-1}\right)\end{array}$ & $\log \mathrm{K}_{\mathrm{ow}}$ & Solubility $\left(\mathrm{mg} \mathrm{L}^{-1}\right)$ & Species & Exposure method & $\begin{array}{l}\text { Duration } \\
\text { (h) }\end{array}$ & $\begin{array}{c}\text { Measured } \\
\mathrm{LC}_{50} \\
\left(\mathrm{mg} \mathrm{L}^{-1}\right) \\
\end{array}$ & $\begin{array}{c}\text { Nominal } \\
\mathrm{LC}_{50} \\
\left(\mathrm{mg} \mathrm{L}^{-1}\right) \\
\end{array}$ & Classification \\
\hline Formic acid & $64-18-6$ & 46.03 & -0.54 & miscible & Leuciscus idus & static & & & 68.00 & 1 \\
\hline Methanamine & $74-89-5$ & 31.06 & -0.57 & $1.00 \mathrm{E}+06$ & $\begin{array}{l}\text { Brachydanio rerio } \\
\text { Leuciscus idus }\end{array}$ & & $\begin{array}{l}96 \\
48\end{array}$ & & $\begin{array}{l}711.00 \\
970.00\end{array}$ & 1 \\
\hline Acetic anhydride & $108-24-7$ & 102.09 & -0.58 & $2.60 \mathrm{E}+04$ & $\begin{array}{l}\text { Leuciscus idus } \\
\text { L }\end{array}$ & & $\begin{array}{l}48 \\
96\end{array}$ & & 265.00 & 1 \\
\hline Nicotinic acid & $59-67-6$ & 123.11 & -0.59 & & Salmo trutta & & 96 & & 520.00 & 1 \\
\hline Lactic acid & $50-21-5$ & 90.10 & -0.62 & $8.76 \mathrm{E}+05$ & $\begin{array}{l}\text { Brachydanio rerio } \\
\text { Lepomis macrochirus }\end{array}$ & & $\begin{array}{l}96 \\
96\end{array}$ & $\begin{array}{l}320.00 \\
130.00\end{array}$ & & 1 \\
\hline 2-Propanol, 1,1'-oxydi- & $110-98-5$ & 134.18 & -0.64 & miscible & Carassius auratus & static & 96 & & $>5000$ & 3 \\
\hline $\mathrm{m}$-Toluenesulfonic acid, 6-amino- & $88-44-8$ & 187.22 & -0.67 & $6.00 \mathrm{E}+03$ & Oryzias Latipes & semi-static & 96 & $>10$ & & 3 \\
\hline Silane, trimethoxymethyl- & $1185-55-3$ & & -0.67 & $1.00 \mathrm{E}+06$ & Oncorhynchus mykiss & flow-through & 96 & $>110$ & & 3 \\
\hline DL-Pantoyl lactone & $79-50-5$ & 130.14 & -0.69 & $5.00 \mathrm{E}+02$ & Cyprinus carpio & semi-static & 96 & & $>140$ & 3 \\
\hline Methyl acetoacetate & $105-45-3$ & & -0.69 & & & & & & & 4 \\
\hline \multirow{2}{*}{ Silicic acid, ethyl ester } & $\begin{array}{l}111-90-0 \\
11099-06-2\end{array}$ & $\begin{array}{l}134.18 \\
10615\end{array}$ & -0.69 & $1.00 \mathrm{E}+06$ & Salmo gairdneri & flow-through & 96 & 13417.00 & & 1 \\
\hline & $11099-06-2$ & & & $1.00 \mathrm{E}+05$ & $\begin{array}{l}\text { Leuciscus idus } \\
\text { melanotus }\end{array}$ & static & 96 & & $\begin{array}{c}>46400< \\
100000\end{array}$ & \\
\hline \multirow[t]{2}{*}{ 2-Butyne-1,4-diol } & $110-65-6$ & 86.09 & -0.73 & $7.50 \mathrm{E}+05$ & $\begin{array}{l}\text { Leuciscus idus } \\
\text { melanotus }\end{array}$ & static & 48 & & 82.00 & 1 \\
\hline & & & & & Pimephales promelas & flow through & 96 & & 53.60 & \\
\hline Methanol & $67-56-1$ & 32.04 & -0.74 & miscible & $\begin{array}{l}\text { Lepomis macrochirus } \\
\text { Salmo gairdneri } \\
\text { Pimephales promelas }\end{array}$ & $\begin{array}{l}\text { flow-through } \\
\text { flow-through } \\
\text { flow-through }\end{array}$ & $\begin{array}{l}96 \\
96 \\
96\end{array}$ & $\begin{array}{l}15,400 \\
20,100 \\
28,100\end{array}$ & & 1 \\
\hline $\begin{array}{l}\text { Ethanol, 2,2'-thiodi- } \\
\text { Phosphoric acid }\end{array}$ & $\begin{array}{l}111-48-8 \\
7664-38-2\end{array}$ & 98.00 & $\begin{array}{l}-0.75 \\
-0.77\end{array}$ & $\begin{array}{c}\text { miscible } \\
5.48 \mathrm{E}+06\end{array}$ & $\begin{array}{l}\text { Leuciscus idus } \\
\text { Oryzias latipes }\end{array}$ & static & $\begin{array}{l}96 \\
96\end{array}$ & 75.10 & $>10000$ & $\begin{array}{l}3 \\
1\end{array}$ \\
\hline Thiophene, tetrahydro-, 1,1-dioxide & $126-33-0$ & 120.17 & -0.77 & $1.00 \mathrm{E}+05$ & $\begin{array}{l}\text { Oryzias latipes } \\
\text { Salmo gairdneri }\end{array}$ & $\begin{array}{l}\text { semi-static } \\
\text { semi-static }\end{array}$ & $\begin{array}{l}96 \\
96\end{array}$ & & $\begin{array}{c}>100 \\
1000.00\end{array}$ & 1 \\
\hline Acetamide, N,N-dimethyl- & $127-19-5$ & 87.12 & -0.77 & miscible & $\begin{array}{l}\text { Oryzias latipes } \\
\text { Leuciscus idus }\end{array}$ & $\begin{array}{l}\text { static } \\
\text { static }\end{array}$ & $\begin{array}{l}48 \\
96\end{array}$ & & $\begin{array}{l}1000.00 \\
>500\end{array}$ & 1 \\
\hline \multirow[t]{2}{*}{ Urea, 1,3-dimethyl- } & $96-31-1$ & & -0.78 & $7.65 \mathrm{E}+05$ & Leuciscus idus & static & 96 & & 10000.00 & 1 \\
\hline & & & & & Danio rerio & static & 96 & & $\begin{array}{c}\geq 1000 \text { to } \\
\leq 2200\end{array}$ & \\
\hline \multirow[t]{2}{*}{ 2-Propanol, 1,1'-iminodi- } & $110-97-4$ & 133.19 & -0.79 & $4.00 \mathrm{E}+05$ & Pimephales promelas & static & 96 & & $>100$ & 3 \\
\hline & & & & & Leuciscus idus & static & 96 & & $\underset{<2200}{\geq 1000 \text { to }}$ & \\
\hline Formamide & $75-12-7$ & 45.04 & -0.82 & $1.00 \mathrm{E}+06$ & $\begin{array}{l}\text { Leuciscus idus } \\
\text { Leuciscus idus }\end{array}$ & $\begin{array}{l}\text { static } \\
\text { static }\end{array}$ & $\begin{array}{l}96 \\
48\end{array}$ & & $\begin{array}{l}\leq 56900 \\
>6300\end{array}$ & 1 \\
\hline Formamide, $\mathrm{N}, \mathrm{N}$-dimethyl- & $68-12-2$ & 73.09 & -0.85 & miscible & $\begin{array}{l}\text { Lepomis macrochirus } \\
\text { Pimephales promelas }\end{array}$ & $\begin{array}{l}\text { flow through } \\
\text { flow through }\end{array}$ & $\begin{array}{l}96 \\
96\end{array}$ & $\begin{array}{c}7100.00 \\
10600.00\end{array}$ & & 1 \\
\hline Stannane, trichloromethyl- & $993-16-8$ & 240.10 & -0.90 & $1.04 \mathrm{E}+06$ & $\begin{array}{l}\text { Brachydanio rerio } \\
\text { Pimephales promelas }\end{array}$ & $\begin{array}{l}\text { semi-static } \\
\text { static }\end{array}$ & $\begin{array}{l}96 \\
96\end{array}$ & $\begin{array}{l}>102 \\
320.00\end{array}$ & & 1 \\
\hline \multirow{2}{*}{$\begin{array}{l}\text { Urea, N,N"-(2-methylpropylidene)bis- } \\
\text { Ethanol, 2-(methylamino)- }\end{array}$} & 6104-30-9 & 174.20 & -0.90 & $3.00 \mathrm{E}+02-3.00 \mathrm{E}+03$ & Oncorhynchus mykiss & static & 96 & & $>1000$ & 3 \\
\hline & $109-83-1$ & & -0.91 & $1.00 \mathrm{E}+06$ & Oncorhynchus mykiss & semi-static & 96 & & 100.00 & 1 \\
\hline Silane, trimethoxy[3-(oxiranylmethoxy)propyl]- & 2530-83-8 & 236.00 & -0.92 & $1.00 \mathrm{E}+06$ & $\begin{array}{l}\text { Oncorhynchus mykiss } \\
\text { Lepomis macrochirus } \\
\text { Cyprinus carpio }\end{array}$ & $\begin{array}{l}\text { static } \\
\text { static } \\
\text { semi-static }\end{array}$ & $\begin{array}{l}96 \\
96 \\
96\end{array}$ & 55.00 & $\begin{array}{l}237.00 \\
276.00\end{array}$ & 1 \\
\hline Ethanol, 2-(dimethylamino)- & $108-01-0$ & 89.14 & -0.94 & miscible & $\begin{array}{l}\text { Leuciscus idus } \\
\text { Pimephales promelas }\end{array}$ & $\begin{array}{l}\text { static } \\
\text { static }\end{array}$ & $\begin{array}{l}96 \\
96\end{array}$ & & $100-200$ & 1 \\
\hline $\begin{array}{l}\text { 1,3-Propanediol, 2-(hydroxymethyl)-2-methyl- } \\
\text { Triethylene glycol, monoethyl ether }\end{array}$ & $77-85-0$ & 178.20 & -0.95 & $\begin{array}{l}3.00 \mathrm{E}+05 \\
\text { soluble }\end{array}$ & $\begin{array}{l}\text { Pimephales promelas } \\
\text { Oryzias latipes }\end{array}$ & $\begin{array}{l}\text { Stanc } \\
\text { semi-static } \\
\text { static }\end{array}$ & 96 & & $\begin{array}{l}81.00 \\
>100 \\
>10000\end{array}$ & 3 \\
\hline \multirow[t]{2}{*}{ 2-Propanol, 1-amino- } & $\begin{array}{l}112-50-5 \\
78-96-6\end{array}$ & 191.27 & $\begin{array}{l}-0.96 \\
-0.96\end{array}$ & soluble & $\begin{array}{l}\text { fathead minnow } \\
\text { Leuciscus idus } \\
\text { Leuciscus idus }\end{array}$ & & $\begin{array}{l}96 \\
96 \\
96\end{array}$ & & $\begin{array}{l}>>10000 \\
>215<464 \\
>1000\end{array}$ & $\begin{array}{l}3 \\
3\end{array}$ \\
\hline & & & & & Pimephales promelas & static & 96 & & 11800.00 & \\
\hline \multirow[t]{2}{*}{ Triethanolamine } & $102-71-6$ & 149.19 & -1.00 & & $\begin{array}{l}\text { Crassius auratus } \\
\text { Leuciscus idus }\end{array}$ & $\begin{array}{l}\text { static } \\
\text { static }\end{array}$ & $\begin{array}{l}96 \\
96\end{array}$ & & $\begin{array}{l}>5000 \\
>10000\end{array}$ & 1 \\
\hline & & & & & $\begin{array}{l}\text { Leuciscus idus } \\
\text { Leuciscus idus }\end{array}$ & $\begin{array}{l}\text { Stancic } \\
\text { static }\end{array}$ & 96 & & $\begin{array}{l}3146600 \\
1466.00\end{array}$ & \\
\hline Ethanol, 2,2'-(methylimino)di- & $105-59-9$ & 119.16 & -1.08 & $1.00 \mathrm{E}+06$ & Cyprinodon variegatus & semi-static & 96 & & $>100$ & 1 \\
\hline Bicyclo[2.2.2]octane, 1,4-diaza- & $280-57-9$ & & -1.13 & $6.10 \mathrm{E}+05$ & Pimephales promelas fry & static & 96 & & 1170.00 & 4 \\
\hline Ethanol, 2-(2-methoxyethoxy)- & $111-77-3$ & 120.15 & -1.18 & & $\begin{array}{l}\text { Pimephales promelas fry } \\
\text { Lepomis macrochirus }\end{array}$ & & $\begin{array}{l}96 \\
96\end{array}$ & & $\begin{array}{l}5700.00 \\
7500.00\end{array}$ & 1 \\
\hline
\end{tabular}


(Table S7. continued)

\begin{tabular}{|c|c|c|c|c|c|c|c|c|c|c|}
\hline Chemicals name & CAS Reg. no & $\begin{array}{c}\text { Molecular } \\
\text { weight } \\
\left(\mathrm{g} \mathrm{mol}^{-1}\right)\end{array}$ & $\log \mathrm{K}_{\mathrm{ow}}$ & Solubility $\left(\mathrm{mg} \mathrm{L}^{-1}\right)$ & Species & Exposure method & $\begin{array}{l}\text { Duration } \\
\text { (h) }\end{array}$ & $\begin{array}{c}\text { Measured } \\
\mathrm{LC}_{50} \\
\left(\mathrm{mg} \mathrm{L}^{-1}\right)\end{array}$ & $\begin{array}{c}\text { Nominal } \\
\text { LC } \\
\left(\mathrm{mg} \mathrm{L}^{-1}\right) \\
\end{array}$ & Classification \\
\hline Dimethyl phosphonate & $868-85-9$ & & -1.20 & $>1.00 \mathrm{E}+05$ & Pimephales promelas & ststic & 96 & & 225.00 & 1 \\
\hline Silane, trimethoxy- & 2487-90-3 & & -1.22 & $1.00 \mathrm{E}+06$ & Oncorhynchus mykiss & static & 96 & & $>100$ & 3 \\
\hline Piperazine & $110-85-0$ & & -1.24 & $1.50 \mathrm{E}+05$ & $\begin{array}{l}\text { Poecilia reticulata } \\
\text { Orvzias latipes }\end{array}$ & $\begin{array}{l}\text { semi-static } \\
\text { Static }\end{array}$ & $\begin{array}{l}96 \\
48\end{array}$ & - & 1000.00 & 3 \\
\hline \multirow{4}{*}{ Diethylenetriamine } & $111-40-0$ & 103.20 & -1.30 & miscible & $\begin{array}{l}\text { Oryzias latipes } \\
\text { Oryzias latipes }\end{array}$ & $\begin{array}{l}\text { Static } \\
\text { semi-static }\end{array}$ & $\begin{array}{l}48 \\
48\end{array}$ & 780.00 & 1000.00 & 1 \\
\hline & & & & & Leuciscus idus & & 96 & 100.00 & 248.00 & \\
\hline & & & & & Pimephales promelas & flow-through & 96 & 34000.00 & & \\
\hline & & & & & Oryzias latipes & Semi-static & 48 & 33000.00 & & \\
\hline \multirow[t]{3}{*}{ Dimethyl sulfoxide } & $67-68-5$ & 78.13 & -1.35 & $1.00 \mathrm{E}+06$ & Salvelinus fontinalis & static & 96 & & 35500.00 & 1 \\
\hline & & & & & Oncorhynchus mykiss & static & 96 & & 32300.00 & \\
\hline & & & & & Lepomis cyanellus & static & 96 & & 43000.00 & \\
\hline \multirow[t]{2}{*}{ Ethylene glycol } & $107-21-1$ & 62.07 & -1.36 & miscible & $\begin{array}{l}\text { Oncorhynchus mykiss } \\
\text { Pimephales promelas }\end{array}$ & $\begin{array}{l}\text { static } \\
\text { static }\end{array}$ & $\begin{array}{l}96 \\
96\end{array}$ & $\begin{array}{l}\text { non-toxic } \\
\text { non-toxic }\end{array}$ & & 3 \\
\hline & & & & & $\begin{array}{l}\text { Leuciscus idus } \\
\text { melanotus }\end{array}$ & & 48 & & $>500$ & \\
\hline Melamine & $108-78-1$ & 126.12 & -1.37 & $3.10 \mathrm{E}+03$ & $\begin{array}{l}\text { Oryzias latipes } \\
\text { Poecilia reticulata }\end{array}$ & & $\begin{array}{l}48 \\
96\end{array}$ & & $\begin{array}{l}1000.00 \\
>3000\end{array}$ & 1 \\
\hline Ammonia & 7664-41-7 & 17.03 & -1.38 & $8.90 \mathrm{E}+05$ & Salmo gairdneri & flow-through & 96 & & $0.163-1.09$ & 1 \\
\hline 7-Amino-4-hydroxy-2-naphthalenesulfonic acid & $87-02-5$ & & -1.39 & $5.00 \mathrm{E}+03$ & & 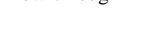 & & & & 4 \\
\hline Triethylene tetramine & $112-24-3$ & 146.23 & -1.40 & miscible & $\begin{array}{l}\text { Poecilia reticulata } \\
\text { Oncorhynchus mykiss }\end{array}$ & $\begin{array}{l}\text { semistatic } \\
\text { static }\end{array}$ & $\begin{array}{l}96 \\
96\end{array}$ & & $\begin{array}{c}570.00 \\
51600.00\end{array}$ & 1 \\
\hline 1,2-Propanediol & $57-55-6$ & 76.09 & -1.41 & soluble & $\begin{array}{l}\text { Pimephales promelas } \\
\text { Pimephales promelas }\end{array}$ & $\begin{array}{l}\text { static } \\
\text { static }\end{array}$ & 96 & & $\begin{array}{l}46500.00 \\
51400.00\end{array}$ & 1 \\
\hline Ethanol, 2-(2-(2-methoxyethoxy)ethoxy)- & $112-35-6$ & 164.20 & -1.46 & soluble & $\begin{array}{l}\text { Pimephales promelas } \\
\text { Fathead minnow }\end{array}$ & $\begin{array}{l}\text { Static } \\
\text { static }\end{array}$ & $\begin{array}{l}96 \\
96\end{array}$ & & $>10000$ & 3 \\
\hline Ethanol, 2-((2-aminoethyl)amino)- & $111-41-1$ & 104.15 & -1.46 & unlimited & $\begin{array}{l}\text { Pimephales promelas } \\
\text { Oryzias latipes }\end{array}$ & $\begin{array}{l}\text { static } \\
\text { static }\end{array}$ & $\begin{array}{r}96 \\
48\end{array}$ & & 640.00 & 1 \\
\hline Ethanol, 2,2'-oxybis- & $111-46-6$ & 106.12 & -1.47 & miscible & Pimephales promelas & $\begin{array}{l}\text { statc } \\
\text { flow-through }\end{array}$ & 96 & 77900.00 & $>1000$ & 1 \\
\hline \multirow[t]{2}{*}{ Piperazine, 1-(2-aminoethyl)- } & $140-31-8$ & 129.20 & -1.48 & miscible & $\begin{array}{l}\text { Leuciscus idus } \\
\text { Pimephales promelas } \\
\text { Pimephales promelas }\end{array}$ & $\begin{array}{l}\text { static } \\
\text { static } \\
\text { static }\end{array}$ & $\begin{array}{l}96 \\
96 \\
96 \\
96\end{array}$ & 2190.00 & $\begin{array}{r}560.00 \\
>100\end{array}$ & 1 \\
\hline & 25265-71-8 & & -1.49 & miscible & Salmo gairdneri & $\begin{array}{l}\text { static } \\
\text { static }\end{array}$ & $\begin{array}{l}96 \\
24\end{array}$ & & $>100$ & 3 \\
\hline \multirow{2}{*}{ Stannane, tetrachloro- } & $7646-78-8$ & 26052 & $\begin{array}{l}-1.49 \\
-153\end{array}$ & water resective & $\begin{array}{l}\text { Brachydanio rerio } \\
\text { Brats }\end{array}$ & $\begin{array}{l}\text { State } \\
\text { Static }\end{array}$ & 96 & 14.00 & (1), & 1 \\
\hline & $1040-18-8$ & 200.52 & -1.53 & water reactive & $\begin{array}{l}\text { Cyprinus carpio } \\
\text { Barilius barna }\end{array}$ & $\begin{array}{l}\text { semistatic } \\
\text { Static }\end{array}$ & $\begin{array}{l}48 \\
96\end{array}$ & & $\begin{array}{l}4.90 \\
>9100\end{array}$ & 1 \\
\hline Urea & $57-13-6$ & 60.06 & -1.59 & $1.08 \mathrm{E}+06$ & $\begin{array}{l}\text { Leuciscus idus } \\
\text { melanotous }\end{array}$ & static & 48 & & $>10000$ & 3 \\
\hline 1,3,5-Triazine-2,4,6(1H,3H,5H)-trione, 1,3,5-tris(2-hydroxyethyl)- & 839-90-7 & & -1.63 & $8.20 \mathrm{E}+05$ & $\begin{array}{l}\text { Oryzias latipes } \\
\text { Leeciscus idus }\end{array}$ & $\begin{array}{l}\text { semi-static } \\
\text { static }\end{array}$ & $\begin{array}{l}96 \\
96\end{array}$ & & $\begin{array}{l}>100 \\
460-680\end{array}$ & 3 \\
\hline Glyoxal & $107-22-2$ & 58.04 & -1.65 & miscible & $\begin{array}{l}\text { Pimephales promelas } \\
\text { Rhombus maximus }\end{array}$ & $\begin{array}{l}\text { static } \\
\text { static }\end{array}$ & $\begin{array}{l}96 \\
96\end{array}$ & $>500$ & 215.00 & 1 \\
\hline 1,2,4-Butanetricarboxylic acid, 2-phosphono- & 37971-36-1 & 270.13 & -1.66 & miscible & Leuciscus idus & static & 96 & & 500.00 & 1 \\
\hline 1,2-Ethanediamine, N-[3-(trimethoxysilyl)propyl]- & $1760-24-3$ & 222.00 & -1.67 & $1.00 \mathrm{E}-06$ & $\begin{array}{l}\text { Lepomis macrochirus } \\
\text { Oncorhvnchus mykiss }\end{array}$ & & $\begin{array}{l}96 \\
96\end{array}$ & & $\begin{array}{l}200.00 \\
213.00\end{array}$ & 2 \\
\hline $\begin{array}{l}\text { 4,4'-Diaminostilbene-2,2'-disulfonic acid } \\
\text { Diazenedicarboxamide }\end{array}$ & $\begin{array}{r}81-11-8 \\
123-77-3\end{array}$ & & $\begin{array}{l}-1.70 \\
-1.70\end{array}$ & $\begin{array}{l}3.20 \mathrm{E}+01 \\
3.54 \mathrm{E}+04\end{array}$ & $\begin{array}{l}\text { Oncornynchus myklss } \\
\text { Oryzias latipes }\end{array}$ & semi-static & $\begin{array}{l}96 \\
96\end{array}$ & & $\begin{aligned} & 213.00 \\
= & >1,000\end{aligned}$ & $\begin{array}{l}3 \\
4\end{array}$ \\
\hline \multirow[t]{2}{*}{ Citric acid } & $77-92-9$ & 192.12 & -1.72 & $5.76 \mathrm{E}+05-7.71 \mathrm{E}+05$ & $\begin{array}{l}\text { Leuciscus idus } \\
\text { Lepomis macrochirus }\end{array}$ & & $\begin{array}{l}96 \\
96\end{array}$ & & $\begin{array}{c}440-760 \\
1516.00\end{array}$ & 1 \\
\hline & & & & & Pimephales promelas & static & 96 & & $>10000$ & \\
\hline 13-Tridecanol, 2,5,8,11-tetraoxa- & $23783-42-8$ & 208.26 & -1.73 & $1.00 \mathrm{E}+06$ & Brachydanio rerio & & $\begin{array}{l}48 \\
96\end{array}$ & & $\begin{array}{l}>10000 \\
>10000\end{array}$ & 3 \\
\hline Triethylene glycol & $112-27-6$ & 150.18 & -1.75 & completely soluble & $\begin{array}{l}\text { Brachydanio rerio } \\
\text { Pimephales promelas }\end{array}$ & flow through & 96 & 69800.00 & $\$ 10000$ & 1 \\
\hline Glycerol & $56-81-5$ & 92.00 & -1.76 & miscible & $\begin{array}{l}\text { Leuciscus idus } \\
\text { melanotus }\end{array}$ & static & 96 & & $>10000$ & 3 \\
\hline $\begin{array}{l}\text { D-Gluconic acid } \\
\text { D-Glucono-1,5-lactone }\end{array}$ & $\begin{array}{l}526-95-4 \\
90-80-2\end{array}$ & $\begin{array}{l}196.16 \\
178.14\end{array}$ & $\begin{array}{l}-1.87 \\
-1.98\end{array}$ & $\begin{array}{l}1.00 \mathrm{E}+03 \\
5.90 \mathrm{E}+05\end{array}$ & & & & & & $\begin{array}{l}4 \\
4\end{array}$ \\
\hline Dipentaerythritol & $126-58-9$ & 254.00 & -2.00 & $3.00 \mathrm{E}+03$ & Oncorhynchus mykiss & semi-static & 96 & & $>100$ & 3 \\
\hline Tetraethylene glycol & $112-60-7$ & 194.23 & -2.02 & miscible & Pimephales promelas & flow-through & 96 & & $>10,000$ & 3 \\
\hline
\end{tabular}


(Table S7. continued)

\begin{tabular}{|c|c|c|c|c|c|c|c|c|c|c|}
\hline Chemicals name & CAS Reg. no & $\begin{array}{c}\text { Molecular } \\
\text { weight } \\
\left(\mathrm{g} \mathrm{mol}^{-1}\right)\end{array}$ & $\log K_{\text {ow }}$ & Solubility $\left(\mathrm{mg} \mathrm{L}^{-1}\right)$ & Species & Exposure method & $\begin{array}{l}\text { Duration } \\
\text { (h) }\end{array}$ & $\begin{array}{c}\text { Measured } \\
\mathrm{LC}_{50} \\
\left(\mathrm{mg} \mathrm{L}^{-1}\right)\end{array}$ & $\begin{array}{c}\text { Nominal } \\
\mathrm{LC}_{50} \\
\left(\mathrm{mg} \mathrm{L}^{-1}\right)\end{array}$ & Classification \\
\hline \multirow[t]{2}{*}{ Ethylenediamine } & \multirow[t]{2}{*}{$107-15-3$} & \multirow[t]{2}{*}{60.10} & \multirow[t]{2}{*}{-2.04} & \multirow[t]{2}{*}{$1.10 \mathrm{E}+05$} & $\begin{array}{l}\text { Pimephales promelas } \\
\text { Pimephales promelas }\end{array}$ & $\begin{array}{l}\text { semi-static } \\
\text { static }\end{array}$ & $\begin{array}{l}96 \\
96\end{array}$ & 115.70 & \multirow[t]{4}{*}{210.00} & 1 \\
\hline & & & & & Poecilia reticulata & semi-static & 96 & 640.00 & & 1 \\
\hline L-Ascorbic acid & $50-81-7$ & 176.12 & -2.05 & $3.30 \mathrm{E}+05$ & Oncorynchus mykiss & 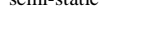 & 96 & $>1000$ & & 3 \\
\hline \multirow[b]{2}{*}{ Stannane, dichlorodimethyl- } & \multirow[b]{2}{*}{$753-73-1$} & \multirow[b]{2}{*}{219.69} & \multirow[b]{2}{*}{-2.18} & \multirow[b]{2}{*}{$8.23 \mathrm{E}+05$} & Brachydanio rerio & semi-static & 96 & $>100$ & & \multirow[b]{2}{*}{1} \\
\hline & & & & & $\begin{array}{l}\text { Pimephales promelas } \\
\text { Cyprinodon variegates }\end{array}$ & $\begin{array}{l}\text { static } \\
\text { static }\end{array}$ & $\begin{array}{l}96 \\
96\end{array}$ & & $\begin{array}{r}320.00 \\
>>1000\end{array}$ & \\
\hline 2,2'-Iminodiethanol & $111-42-2$ & 105.14 & -2.18 & \multirow{3}{*}{ miscible } & Pimephales promelas & static & 96 & & 1370.00 & 1 \\
\hline & $111-42-2$ & 105.14 & -2.18 & & Gambusia affinis & static & 96 & & 1400.00 & 1 \\
\hline $\begin{array}{l}\text { 2-Imidazolidinone, 4,5-dihydroxy-1,3-bis } \\
\text { D-Glucose }\end{array}$ & $\begin{array}{l}1854-26-8 \\
50-99-7\end{array}$ & $\begin{array}{l}178.14 \\
180.16\end{array}$ & $\begin{array}{l}-2.20 \\
-2.20\end{array}$ & & Pimephales promelas & \multirow[t]{2}{*}{ low priority } & 96 & & 2200.00 & $\begin{array}{l}1 \\
4\end{array}$ \\
\hline Sulfuric acid & 7664-93-9 & 98.07 & -2.21 & miscible & Lepomis macrochirus & & 96 & & \multirow{2}{*}{$\begin{array}{l}16-28 \\
82.00\end{array}$} & 1 \\
\hline Ethanol, 2-amino- & $141-43-5$ & 61.08 & -2.30 & & $\begin{array}{l}\text { Brachyaanolo rerio } \\
\text { Carassius auratus }\end{array}$ & & 96 & 10.00 & & 1 \\
\hline Pentaethylene glycol & $4792-15-8$ & 238.28 & -2.30 & $1.00 \mathrm{E}+06$ & Pimephales promelas & \multirow[t]{3}{*}{ flow-through } & 96 & $>50,000$ & & 3 \\
\hline Trimethyl methylamine hydroxide & $75-59-2$ & 91.15 & -2.47 & \multirow{2}{*}{$\begin{array}{l}1.00 \mathrm{E}+06 \\
\text { miscible }\end{array}$} & & & & & 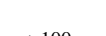 & 4 \\
\hline Morpholine & $110-91-8$ & 87.12 & -2.55 & & Oryzias latipes & & 96 & & \multirow{3}{*}{$\begin{array}{l}75.00 \\
75.00\end{array}$} & 3 \\
\hline Maleic anhydride & $108-31-6$ & 98.06 & -2.61 & \multirow{2}{*}{$4.00 \mathrm{E}+05$} & Salmo gairdneri & \multirow{2}{*}{$\begin{array}{l}\text { static } \\
\text { static }\end{array}$} & 96 & & & 1 \\
\hline D-Glucitol & $50-70-4$ & 182.17 & -3.10 & & Lepomis macrochirus & & & & & 4 \\
\hline Tetraethylenepentamine & $112-57-2$ & 189.30 & -3.16 & $1.00 \mathrm{E}+06$ & Poecilia reticulata & \multirow{4}{*}{$\begin{array}{l}\text { semi-static } \\
\text { static }\end{array}$} & 96 & \multirow{4}{*}{$\begin{array}{l}310.00 \\
416.00\end{array}$} & \multirow[t]{4}{*}{420.00} & 1 \\
\hline Methanesulfinic acid, aminoimino- & $1758-73-2$ & 108.12 & -3.23 & \multirow{3}{*}{$2.70 \mathrm{E}+04$} & $\begin{array}{l}\text { Pimephales promelas } \\
\text { Poecilia reticulata }\end{array}$ & & $\begin{array}{l}96 \\
96\end{array}$ & & & 1 \\
\hline Syrups, hydrolyzed starch & $8029-43-4$ & 180.16 & -3.24 & & & & & & & 4 \\
\hline Glycine, N,N'-1,3-propanediylbis[N-(carboxymethyl)- & 1939-36-2 & 306.27 & -3.37 & & & & & & & 4 \\
\hline Phosphonic acid, [nitrilotris(methylene)]tris- & 6419-19-8 & 299.07 & -3.53 & \multirow{2}{*}{$\begin{array}{l}6.10 \mathrm{E}+05 \\
8.64 \mathrm{E}+03\end{array}$} & Oncorhynchus mykiss & static & 48 & & \multirow{3}{*}{$\begin{array}{c}4344.50 \\
8132.00 \\
>100\end{array}$} & 1 \\
\hline Glutamic acid & $56-86-0$ & 147.13 & -369 & & $\begin{array}{l}\text { Cyprimedon variegatus } \\
\text { Orzzias latipes }\end{array}$ & $\begin{array}{l}\text { static } \\
\text { static }\end{array}$ & $\begin{array}{l}96 \\
96\end{array}$ & & & 3 \\
\hline Sucrose & $57-50-1$ & 342.30 & -3.70 & \multirow[b]{2}{*}{$6.67 \mathrm{E}+05$} & & low priority & & & & 4 \\
\hline Methenamine & $100-97-0$ & 140.19 & -4.15 & & $\begin{array}{l}\text { Cyprinodon variegatus } \\
\text { Pimenhales vremelus }\end{array}$ & $\begin{array}{l}\text { static } \\
\text { flow-through }\end{array}$ & 96 & 49800000 & 49000.00 & 1 \\
\hline E.D.T.A & $60-00-4$ & 292.30 & -5.01 & $4.00 \mathrm{E}+02$ & & & & & & 4 \\
\hline $\begin{array}{l}\text { Phosphonic acid, [[(phosphonomethyl)imino]bis[2,1- } \\
\text { ethanediylnitrilobis(methylene)]]tetrakis- }\end{array}$ & $15827-60-8$ & 573.20 & -9.72 & $5.00 \mathrm{E}+05$ & $\begin{array}{l}\text { Oncorhynchus mykiss } \\
\text { Ictalurus punctatus }\end{array}$ & $\begin{array}{l}\text { static } \\
\text { static }\end{array}$ & $\begin{array}{l}96 \\
96 \\
96\end{array}$ & & $\begin{array}{l}180-252 \\
657.00\end{array}$ & 1 \\
\hline $\begin{array}{l}\text { Maltodextrin } \\
\text { Hexafluorosilicic acid }\end{array}$ & $\begin{array}{l}9050-36-6 \\
16961-83-4\end{array}$ & $\begin{array}{l}342.30 \\
144.09\end{array}$ & & & Lepomis macrochirus & static & & & & $\begin{array}{l}4 \\
4\end{array}$ \\
\hline
\end{tabular}


Table S8. Outliers of the proposed model with their physico-chemical properties and $\mathrm{LC}_{50}$ or $\mathrm{EC}_{50}$ values

\begin{tabular}{|c|c|c|c|c|c|c|}
\hline Chemical name & CAS Reg. no. & Structure & $\log \mathrm{K}_{\mathrm{ow}}$ & $\begin{array}{c}\text { Water } \\
\text { solubility } \\
\left(\mathrm{mg} \mathrm{L}^{-1}\right) \\
\end{array}$ & $\begin{array}{l}\mathrm{LC}_{50} \text { or } \mathrm{EC}_{50} \\
\quad\left(\mathrm{mg} \mathrm{L}^{-1}\right)\end{array}$ & Test species \\
\hline $\begin{array}{l}\text { Triisooctyl } 2,2^{\prime}, 2 "- \\
{[(\text { octylstannylidyne)tris(thio)]triacetate }}\end{array}$ & $26401-86-5$ & & 14.4 & $0.5 \sim 2.7$ & $\begin{array}{c}1.65 \\
1^{*} \\
2.3\end{array}$ & $\begin{array}{l}\text { P. subspicatus } \\
\text { D. magna } \\
\text { B. rerio }\end{array}$ \\
\hline $\begin{array}{l}\text { 2-Ethylhexyl 10-ethyl-4-[[2-[(2- } \\
\text { ethylhexyl)oxy]-2-oxoethyl]thio]-4-octyl-7- } \\
\text { oxo-8-oxa-3,5-dithia-4-stannatetradecanoate }\end{array}$ & $27107-89-7$ & & 14.1 & $0.5 \sim 2.7$ & $0.3-3.1^{*}$ & $\begin{array}{l}\text { P. subspicatus } \\
\text { D. magna }\end{array}$ \\
\hline $\begin{array}{l}\text { 8-Oxa-3,5-dithia-4-stannatetradecanoic acid, } \\
\text { 4,4-dibutyl-10-ethyl-7-oxo-, 2-ethylhexyl ester }\end{array}$ & $10584-98-2$ & & 11.43 & 0.32 & $0.035^{*}$ & D. magna \\
\hline Stannane, tetrabutyl- & $1461-25-2$ & & 9.37 & $<0.1$ & $\begin{array}{c}0.045 \\
0.05\end{array}$ & $\begin{array}{l}\text { P. promelas } \\
\text { S. costatum } \\
\end{array}$ \\
\hline 1-Hexadecen-3-ol, 3,7,11,15-tetramethyl- & $505-32-8$ & & 8.1 & 5.8 & 0.13 & D. magna \\
\hline Benzene, undecyl- & $6742-54-7$ & & 7.45 & 0.041 & $0.009^{*}$ & D. magna \\
\hline Phenol, tetrapropylene & $57427-55-1$ & & 7.14 & - & $\begin{array}{l}0.091 \\
0.017\end{array}$ & $\begin{array}{l}\text { P. subspicatus } \\
\text { D. magna }\end{array}$ \\
\hline Bis(2-ethylhexyl) phthalate & $117-81-7$ & & 7.00 & 1.3 & $0.133^{*}$ & D. pulex \\
\hline Trisiloxane, octamethyl- & $107-51-7$ & & 6.6 & 0.034 & $0.0094^{*}$ & P. subspicatus \\
\hline Methyl laurate & $111-82-0$ & & 6.5 & 1.39 & 0.23 & D. magna \\
\hline
\end{tabular}


(Table S8. continued)

\begin{tabular}{|c|c|c|c|c|c|c|}
\hline Chemical name & CAS Reg. no. & Structure & $\log \mathrm{K}_{\text {ow }}$ & $\begin{array}{c}\text { Water } \\
\text { solubility } \\
\left(\mathrm{mg} \mathrm{L}^{-1}\right)\end{array}$ & $\begin{array}{l}\mathrm{LC}_{50} \text { or } \mathrm{EC}_{50} \\
\quad\left(\mathrm{mg} \mathrm{L}^{-1}\right)\end{array}$ & Test species \\
\hline $\begin{array}{l}\text { Decanedioic acid, bis(2,2,6,6-tetramethyl-4- } \\
\text { piperidinyl) ester }\end{array}$ & $52829-07-9$ & & 6.5 & 18.8 & $\begin{array}{l}1.9^{*} \\
17^{*} \\
4.3\end{array}$ & $\begin{array}{l}\text { P. subspicatus } \\
\text { D. magna } \\
\text { O. mykiss }\end{array}$ \\
\hline $\begin{array}{l}\text { 1,1'-(1,1-dimethyl-3-methylene-1,3- } \\
\text { propanediyl)bisbenzene }\end{array}$ & $6362-80-7$ & & 6.2 & 0.23 & 0.057 & D. magna \\
\hline tert-Dodecanethiol & $25103-58-6$ & & 6.2 & 0.25 & 0.16 & D. magna \\
\hline 1-Dodecanethiol & $112-55-0$ & $\mathrm{H}_{3} \mathrm{C}^{\prime}$ & 6.2 & 1.0 & $<0.0145$ & P. subspicatus \\
\hline
\end{tabular}

nominal concentration 


\section{REFERENCES}

(1) Mailhot, H. Prediction of algal bioaccumulation and uptake rate of nine organic compounds by ten physicochemical properties. Environ. Sci. Technol. 1987, 21(10), 1009-1013.

(2) Jessiman, B. J.; Qadri, S. U. Bioaccumulation kinetics of the organochlorine pesticide mirex in amphipods. Ecotoxicol. Environ. Saf. 1983, 7(3), 295-305.

(3) van Hattum, B.; Cid Montañés, J. F. C. Toxicokinetics and bioconcentration of polycyclic aromatic hydrocarbons in freshwater isopods. Environ. Sci. Technol. 1999, 33(14), 24092417.

(4) Nikkila, A.; Paulsson, M.; Almgren, K.; Blanck, H.; Kukkonen, J. V. K. Atrazine uptake, elimination, and bioconcentration by peryphyton communities and Daphnia: effects of dissolved organic carbon. Environ. Toxicol. Chem. 2001, 20(5), 1003-1011.

(5) Landrum, P. F. Toxicokinetics of organic xenobiotics in the amphipod, Pontoporeia hoyi: role of physiological and environmental variables. Aquat. Toxicol. 1988, 12(3), 245-271.

(6) Meadows, J. C.; Echols, K. R.; Huckins, J. N.; Borsuk, F. A.; Carline, R. F.; Tillitt, D. E. Estimation of uptake rate constants for PCB congeners accumulated by semipermeable membrane devices and brown trout (Salmo trutta). Environ. Sci. Technol. 1998, 32(12), $1847-1852$.

(7) Lu, Y.; Wang, Z. Accumulation of organochlorinated pesticides by triolein-containing semipermeable membrane device (triolein-SPMD) and rainbow trout. Water Res. 2003, 37(10), 2419-2425. 
(8) Oliver, B. G.; Niimi, A. J. Bioconcentration factors of some halogenated organics for rainbow trout: Limitations in their use for prediction of environmental residues. Environ. Sci. Technol. 1985, 19(9), 842-849.

(9) Baussant, T.; Sanni, S.; Skadsheim, A.; Jonsson, G.; Børseth, J. F.; Gaudebert, B. Bioaccumulation of polycyclic aromatic compounds: 2. Modeling bioaccumulation in marine organisms chronically exposed to dispersed oil. Environ. Toxicol. Chem. 2001, 20(6), 1185-1195.

(10) Fox, K.; Zauke, G. P.; Butte, W. Kinetics of bioconcentration and clearance of 28 polychlorinated biphenyl congeners in zebrafish (Brachydanio rerio). Ecotoxicol. Environ. Saf. 1994, 28(1), 99-109.

(11) Butte, W.; Fox, K.; Zauke, G.-P. Kinetics of bioaccumulation and clearance of isomeric hexachlorocyclohexanes. Sci. Total Environ. 1991, 109-110, 377-382.

(12) Bruggeman, W. A.; Martron, L. B. J. M.; Kooiman, D.; Hutzinger, O. Accumulation and elimination kinetics of di-, tri- and tetra chlorobiphenyls by goldfish after dietary and aqueous exposure. Chemosphere 1981, 10(8), 811-832.

(13) Banerjee, S.; Sugatt, R. H.; O'Grady, D. P. A smiple method for determining bioconcentration parameters of hydrophobic compounds. Environ. Sci. Technol. 1984, 18(2), $79-81$.

(14) Bradbury, S.; Dady, J. M.; Fitzsimmons, P. N.; Volt, M. M.; Hammermeister, D. E.; Erickson, R. J. Toxicokinetics and metabolism of aniline and 4-chloroaniline in medaka (Oryzias latipes). Toxicol. Appl. Pharmacol. 1993, 118(2), 205-214. 
(15) Chaisuksant, Y.; Yu, Q.; Connell, D. W. Bioconcentration of bromo- and chlorobenzenes by fish (Gambusia affinis). Wat. Res. 1997, 31(1), 61-68.

(16) de Wolf, W.; Mast, B.; Yedema, E. S. E.; Seinen, W.; Hermens, J. L. M. Kinetics of 4chloroaniline in guppy, Poecilia reticulata. Aquatic Toxicol. 1994, 28(1-2), 65-78.

(17) de Wolf, W.; Yedema, E. S. E.; Seinen, W.; Hermens, J. L. M. Bioconcentration kinetics of chlorinated anilines in gyppy, Poecilia reticulata. Chemosphere 1994, 28(1), 159-167.

(18) Ensenbach, U.; Nagel, R. Toxicokinetics of xenobiotics in zebrafish- comparison between tap and river water. Comp. Biochem. Physiol. C 1991, 100(1-2), 49-53.

(19) Ensenbach, U.; Hryk, R.; Nagel, R. Kinetics of 3,4-dichloroaniline in several fish species exposed to different types of water. Chemosphere 1996, 32(8), 1643-1654.

(20) Kalsch, W.; Nagel, R.; Urich, K. Uptake, elimination, and bioconcentration of ten anilines in zebrafish (Brachydanio rerio). Chemosphere 1991, 22(3-4), 351-363.

(21) Könemann, H.; van Leeuwen, K. Toxicokinetics in fish: accumulation and elimination of six chlorobenzenes by guppies. Chemosphere 1980, 9(1), 3-19.

(22) Lang, P.-Z.; Wang, Y.; Chen, D.-B.; Wang, N.; Zhao, X.-M.; Ding, Y.-Z. Bioconcentrationm elimination and metabolism of 2,4-dinitrotoluene in carps (Cyprinus Carpio L.). Chemosphere 1997, 35(8), 1799-1815.

(23) Sijm, D.T.H.M.; van der Linde, A. Size-dependent bioconcentration kinetics of hydrophobic organic chemicals in fish based on diffusive mass transfer and allometric relationships. Environ. Sci. Technol. 1995, 29(11), 2769-2777. 
(24) Smith, A.D.; Bharath, A.; Mallard, C.; Orr, D.; McCarty, L. S.; Ozburn, G. W. Bioconcentration kinetics of some chlorinated benzenes and chlorinated phenols in American flagfish, Jordanella floridae (Goode and Bean). Chemosphere 1990, 20(3-4), 379386.

(25) Spacie, A.; Landrum, P. F.; Leversee, G. J. Uptake, depuration, and biotransformation of anthracene and benzo[a]pyrene in bluegill sunfish. Ecotoxicol. Environ. Saf. 1983, 7(3), 330341.

van Eck, J. M. C.; Koelmans, A. A.; Deneer, J. W. Uptake and elimination of 1,2,4trichlorobenzene in the guppy (Poecilia reticulate) at sublethal and lethal aqueous concentrations. Chemosphere 1997, 34(11), 2259-2270.

(27) de Wolf, W.; Lieder, P. H. A novel method to determine uptake and elimination kinetics of volatile chemicals in fish. Chemosphere 1998, 36(8), 1713-1724.

(28) Djomo, J. E.; Garrigues, P.; Narbonne, J. F. Uptake and depuration of polycyclic aromatic hydrocarbons from sediment by the zebrasifh (Brachydanio rerio). Environ. Toxicol. Chem. 1996, 14(7), 1177-1181.

(29) McCarthy, J. F.; Jimenez, B. D. Reduction in bioavailability to bluegills of polycyclic aromatic hydrocarbons bound to dissolved humic material. Environ. Toxicol. Chem. 1985, $4(4), 511-521$.

(30) Linder, G.; Bergman, H. L.; Meyer, J. S. Anthracene bioconcentration in rainbow trout during single-compound and complex-mixture exposures. Environ. Toxicol. Chem. 1985, $4(4), 549-558$. 
(31) Jonsson, G.; Bechmann, R. K.; Bamber, S. D.; Baussant, T. Bioconcentration, biotransformation, and elimination of polycyclic aromatic hydrocarbons in sheepshead minnows (Cyprinodon varigatus) exposed to contaminated seawater. Environ. Toxicol. Chem. 2004, 23(7), 1538-1548.

(32) Gobas, F. A. P. C.; Lovett-Doust, L.; Haffner, G. D. A comparative study of the bioconcentration and toxicity of chlorinated hydrocarbons in aquatic macrophytes and fish. In Plants for toxicity assessment, 2nd, ASTM STP 1115. Gorsuch, J. W., Lower, W. R., St. John, K. R., Eds., American Society for Testing and Materials, Philadelphia, 1991, pp. 178193.

(33) Becker, E. W. Microalgae: Biotechnology and Microbiology; Cambridge University Press: New York, USA, 1994.

(34) Southworth, G. R.; Beauchamp, J. J.; Schmieder, P. K. Bioaccumulation potential of polycyclic aromatic hydrocarbons in Daphnia pulex. Water Res. 1978, 12(11), 973-977.

(35) Ellgehausen, H.; Guth, J. A.; Esser, H. O. Factors determining the bioaccumulation potential of pesticides in the individual compartments of aquatic food chains. Ecotoxicol. Environ. Safety 1980, 4(2), 134-157.

(36) Gobas, F. A. P. C.; Muir, D. C. G.; Mackay, D. Dynamics of dietary bioaccumulation and faecal elimination of hydrophobic organic chemicals in fish. Chemosphere 1988, 17(5), 943962. 